\title{
Análise das bifurcações de um sistema de dinâmica de populações
}

\author{
André Ricardo Belotto da Silva
}

\author{
DissertaÇÃO APRESENTADA \\ $\mathrm{AO}$ \\ Instituto De Matemática e Estatística \\ DA \\ Universidade DE SÃo PAUlO \\ PARA \\ OBTENÇÃO DO TÍTULO \\ $\mathrm{DE}$ \\ Mestre em CiênCias
}

Programa: Matemática Aplicada

Orientador: Prof. Dr. Jorge Manuel Sotomayor Tello

Durante o desenvolvimento deste trabalho o autor recebeu auxílio financeiro da FAPESP

São Paulo, julho de 2010 


\title{
Análise das bifurcações de um sistema de dinâmica de populações
}

\author{
Esta versão definitiva da dissertação \\ contém as correções e alterações sugeridas pela \\ Comissão Julgadora durante a defesa realizada \\ por André Ricardo Belotto da Silva em 16/7/2010.
}

Comissão Julgadora:

- Prof. Dr. Jorge Manuel Sotomayor Tello (orientador) - IME-USP

- Prof. Dr. Pedro Antonio Santoro Salomão - IME-USP

- Prof. Dr. Ronaldo Alves Garcia - UFG 


\section{Agradecimentos}

Agradeço ao meu orientador, Jorge Manuel Sotomayor Tello, pelos conselhos, lições e pela presença durante os últimos anos, nunca me deixando desamparado diante das dificuldades do projeto. Sua sabedoria e conhecimento serviram de inspiração.

Agradeço à minha mãe, ao meu pai e à minha irmã, por sempre me darem apoio e incentivo. Certamente eles formaram o principal pilar para meu progresso acadêmico e pessoal. Minha mãe pela sua leveza e sabedoria, meu pai pela ética e paciência e minha irma pela amizade e determinação. Agradeço também à Amanda Paulino pelo amor e carinho que me proporcionou durante os últimos dois anos e meio. Sem ela o tempo do trabalho não teria sido tão doce.

Agradeço também aos meus amigos do IME que me inspiraram, motivaram e dividiram comigo as mesmas angústias e prazeres de uma pós-graduação. Em especial, agradeço ao Arlane da Silva, por me mostrar o valor da disciplina, ao Bernard Berkovitz, por ser o amigo de todas as horas e ao Cleber e Gustavo, por me ajudarem a vencer a difícil luta contra o computador.

Por fim, agradeço a todos os professores do meu curso de mestrado e de graduação, com especial ênfase aos professores José Verderesi, Pedro Salomão e André Gomes pelos excelentes e agradáveis cursos que ministraram, contribuindo em muito para minha formação matemática. 


\section{Resumo}

Nesta dissertação, tratamos do estudo das bifurcações de um modelo bi-dimensional de presapredador, que estende e aperfeiçoa o sistema de Lotka-Volterra. Tal modelo apresenta cinco parâmetros e uma função resposta não monotônica do tipo Holling IV:

$$
\left\{\begin{array}{c}
\dot{x}=x\left(1-\lambda x-\frac{y}{\alpha x^{2}+\beta x+1}\right) \\
\dot{y}=y\left(-\delta-\mu y+\frac{x}{\alpha x^{2}+\beta x+1}\right)
\end{array}\right.
$$

Estudamos as bifurcações do tipo sela-nó, Hopf, transcrítica, Bogdanov-Takens e BogdanovTakens degenerada. O método dos centros organizadores é usado para estudar o comportamento qualitativo do diagrama de bifurcação.

Palavras-chave: Bifurcação, Predador-presa, Lotka-Volterra, Função resposta Holling IV, Centros organizadores, Hopf, Bogdanov-Takens, Bogdanov-Takens degenerado, Elíptica-nilpotente, Foco-nilpotente . 


\section{Abstract}

In this work are studied the bifurcations of a bi-dimensional predator-prey model, which extends and improves the Volterra-Lotka system. This model has five parameters and a non-monotonic response function of Holling IV type:

$$
\left\{\begin{array}{c}
\dot{x}=x\left(1-\lambda x-\frac{y}{\alpha x^{2}+\beta x+1}\right) \\
\dot{y}=y\left(-\delta-\mu y+\frac{x}{\alpha x^{2}+\beta x+1}\right)
\end{array}\right.
$$

They studied the sadle-node, Hopf, transcritic, Bogdanov-Takens and degenerate BogdanovTakens bifurcations. The method of organising centers is used to study the qualitative behavior of the bifurcation diagram.

Keywords: Bifurcation, Predator-prey, Lotka-Volterra, Holling IV response funciton, Organising centers, Hopf, Bogdanov-Takens, Degenerate Bogdanov-Takens, Nilpotent eliptic, Nilpotent focus . 


\section{Índice}

1 Introdução 1

1.1 O modelo biológico . . . . . . . . . . . . . . . . . . . . . . 2

1.1.1 Evolução dos modelos presa-predador . . . . . . . . . . . . 3

1.1 .2 O modelo escolhido . . . . . . . . . . . . . . . 5

1.2 Bifurcações . . . . . . . . . . . . . . . . . . . . 6

1.2.7 Bifurcações encontradas no modelo . . . . . . . . . . . . . . . . . . . 9

1.3 Esquema dos resultados deste trabalho . . . . . . . . . . . . . . . 10

1.3.6 Organização do trabalho . . . . . . . . . . . . . . . . 13

2 Estudo inicial do sistema $\quad 15$

2.1 Simplificando o sistema . . . . . . . . . . . . . . . . 15

2.2 Breve estudo sobre a função resposta . . . . . . . . . . . . . . . 16

2.3 Domínio de captura . . . . . . . . . . . . . . . . . . . . . 18

2.4 Estudo da posição dos equilíbrios . . . . . . . . . . . . . . . . . . . . . 20

3 Estudo da bifurcação transcrítica de C $\quad 21$

3.1 A bifurcação transcrítica . . . . . . . . . . . . . . . . . . 22

3.1 .1 Estratégia . . . . . . . . . . . . . . . . 22

3.1 .2 A função $G(x)$. . . . . . . . . . . . . . . . . . . . 23

3.1.3 Estudo da condição de não-degenerescência . . . . . . . . . . . . . . . 24

3.1.4 Estudo dos $T C$ 's . . . . . . . . . . . . . . . . . . . . . 25

3.1.5 Estudo das condições de transversalidade . . . . . . . . . . . . . 27

3.2 Estudo da variedade central associada a $C \ldots \ldots$. . . . . . . . . . . 29 
3.2.1 Calculo de $\Gamma_{1}, \Gamma_{2}$ e $\Gamma_{3} \ldots \ldots \ldots \ldots$

3.3 Diagrama de bifurcação próximo a $C \ldots \ldots$. . . . . . . . . . . 32

3.3.1 Os estratos . . . . . . . . . . . . . . . . . . . 32

3.3.2 A configuração topológica local de $C$. . . . . . . . . . . . . 34

3.3.4 Análise da curva $S N_{2}$ subordinada a $T C_{3} \ldots \ldots$. . . . . . . . . . 37

3.4 Estudo da bifurcação transcrítica em $C \operatorname{com} \beta>0 \ldots$. . . . . . . . . . 40

4 Estudo das bifurcações do tipo sela-nó

4.1 Estratégia . . . . . . . . . . . . . . . . . . . 43

4.2 Estudo de $\Psi_{1} \quad \ldots \ldots \ldots \ldots$

$4.3 S N_{1} \ldots \ldots \ldots \ldots \ldots$

4.4 Estudo de $\Psi_{2} \ldots \ldots \ldots \ldots \ldots$

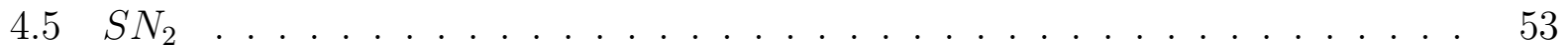

4.6 Análise de $\left(F(x), F^{\prime}(x), F^{\prime \prime}(x), F^{\prime \prime \prime}(x)\right) \ldots \ldots \ldots \ldots$

4.7 Conclusão . . . . . . . . . . . . . . . . . . . . . . 55

5 Estudo sobre as bifurcações de Hopf $\quad 57$

5.1 Estratégia . . . . . . . . . . . . . . . . . . 57

5.2 Forma Normal . . . . . . . . . . . . . . . . . . . 58

5.3 Estudo de $\mathcal{H}_{1} \ldots \ldots \ldots \ldots$. . . . . . . . . . . . . . . . . 60

5.4 Superfície $H_{1} \quad \ldots \ldots \ldots \ldots$

5.5 Conjectura sobre a curva $H_{2} \ldots \ldots \ldots$. . . . . . . . . . . . . . 65

5.6 Conjectura sobre bifurcações de Hopf mais degeneradas . . . . . . . . . . . . 67

5.7 Conclusões . . . . . . . . . . . . . . . . . . . . 67

5.8 Apêndice - Valores dos coeficientes . . . . . . . . . . . . . 68

6 Bifurcações no interior de $Q \quad 73$

6.1 Estudo da região de equilíbrios . . . . . . . . . . . . . . . 73

6.2 Equilíbrios onde det $=0 \ldots \ldots \ldots \ldots$. . . . . . . . . . . . . . . . .

6.2.3 Estratégia . . . . . . . . . . . . . . 79

6.2 .4 Parametrização dos ramos . . . . . . . . . . . . . 80 
6.2 .10 Estudo da função disc . . . . . . . . . . . . . . . . . . . . . 82

6.2.17 Estudo preliminar da intersecção de $W=0 \operatorname{com} \mathcal{S}$. . . . . . . . . 88

6.2.21 Estudo da conexidade de $\gamma_{2}$ nos parâmetros admissíveis . . . . . . . . 90

6.2.26 Estudo sobre a dobra geral . . . . . . . . . . . . . . . . . 95

6.3 Equilíbrios com $t r=0 \ldots \ldots \ldots$. . . . . . . . . . . . . 98

6.4 Estudo dos equilíbrios hiperbólicos . . . . . . . . . . . . . . 100

7 Estudo das bifurcações de Bogdanov-Takens 105

7.1 Lemas Preliminares . . . . . . . . . . . . . . . . . 106

7.2 Estudo da superfície de candidatos a Bogdanov-Takens . . . . . . . . . . 108

7.3 Forma Normal preliminar . . . . . . . . . . . . . . . . . . 120

7.4 Estudo da forma normal de $B T_{3} \ldots \ldots$. . . . . . . . . . . . . . . . . . 122

7.5 Estudo da forma normal de $N F_{3}$ e $N E_{3} \quad \ldots \ldots \ldots$. . . . . . . . . . . 124

7.6 Estudo algébrico das condições de não-degenerescência. . . . . . . . . . . . . 129

7.7 Condições de transversalidade . . . . . . . . . . . . . . . . . . . 139

7.8 Estudo da posição geométrica dos centros organizadores. . . . . . . . . . . 145

7.9 Validade dos centros organizadores . . . . . . . . . . . . . . . . 152

7.10 Conclusões e conjecturas do capítulo . . . . . . . . . . . . . . 163

8 Resultados complementares $\quad 167$

8.1 Número de equilíbrios . . . . . . . . . . . . . . . . . 167

8.2 Resultados de outros autores . . . . . . . . . . . . . . . . . . 172

8.3 Diagrama de Bifurcação . . . . . . . . . . . . . . . . . . 177

9 Conclusões 183

9.1 Resultados válidos para $\mathcal{W} \times \Delta \ldots \ldots \ldots$. . . . . . . . . . . 184

9.2 Resultados demonstrados para $\mathcal{W}_{1} \times \Delta \ldots \ldots \ldots \ldots$

9.3 Problemas que ficaram em aberto . . . . . . . . . . . . . . . 186

9.4 Últimas considerações . . . . . . . . . . . . . . . . . . . . . . . . 187

10 Apêndice $\quad 189$

10.1 Transcrítica de codimensão $3 \ldots$. . . . . . . . . . . . . . . 189 
10.2 Bogdanov-Takens . . . . . . . . . . . . . . . . . . . 190

10.3 Bogdanov-Takens degenerada . . . . . . . . . . . . . . . . . 190

10.4 Foco-Nilpotente . . . . . . . . . . . . . . . . . . . . . . 192

10.5 Elíptica-Nilpotente . . . . . . . . . . . . . . . . . . . 192

$\begin{array}{ll}\text { Referências Bibliográficas } & 196\end{array}$ 


\section{Capítulo 1}

\section{Introdução}

A teoria das bifurcações focaliza o estudo de sistemas dinâmicos nos quais existem parâmetros e a variação em relação eles é "contínua". Em particular podemos tratar de campos vetoriais que dependem de parâmetros. Nesse caso o espaço de fase do sistema pode ter configurações topológicas completamente distintas, dependendo dos parâmetros fixados. A teoria de bifurcações é uma importante ferramenta para atacar essa questão, possibilitando a divisão do espaço de parâmetros em conjuntos nos quais as configurações topológicas sejam iguais, além de auxiliar no próprio estudo das configurações topológicas. Isso é importante para a utilização e a interpretação de modelos, sendo eles provenientes da própria matemática, ou de outras áreas.

Neste trabalho estudaremos um modelo de dinâmica populacional que prevê defesa de grupo. Tal modelo é uma variação dos modelos de Lotka-Volterra e tem como diferencial uma diminuição na quantidade de caça quando a quantidade de presas cresce muito. Apesar de contra-intuitivo, isso ocorre em diversos eco-sistemas. Este modelo consiste em um sistema de equações diferenciais com cinco parâmetros:

$$
\left\{\begin{aligned}
\dot{x}=x\left(1-\lambda x-\frac{y}{\alpha x^{2}+\beta x+1}\right) & =f_{1}(x, y, \alpha, \beta, \mu, \delta, \lambda) \\
\dot{y}=y\left(-\delta-\mu y+\frac{x}{\alpha x^{2}+\beta x+1}\right) & =f_{2}(x, y, \alpha, \beta, \mu, \delta, \lambda)
\end{aligned}\right.
$$

Como queremos tratar aspectos qualitativos, utilizar a teoria de bifurcações é interessante. Assim, vamos estudar e classificar todas as possíveis bifurcações que ocorrem neste sistema. 
Além disso, obteremos informações sobre a localização e o comportamento dos estratos do diagrama de bifurcação. Para isso usaremos o método de centros organizadores. Este método consiste em procurar as bifurcações de codimensão mais alta e usar a teoria geral para entender o comportamento local, possibilitando uma ideia da configuração global no diagrama de bifurcação.

O primeiro estudo deste modelo foi realizado por Saleh $[1,2,3]$. Ele usa conceitos da teoria da bifurcação para explicitar a dinâmica, apresentando uma metodologia que pode ser aplicada para o estudo de diversos outros modelos.

Nesta dissertação nos concentraremos no estudo das bifurcações deste modelo. Assim, apresentaremos um exemplo da aplicação do método dos centros organizadores e estudaremos o sistema biológico proposto. De forma a completar o estudo iniciado por Saleh, vamos provar de forma algébrica e analítica alguns dos resultados que este autor aborda computacionalmente.

\subsection{O modelo biológico}

Antes de começar a discutir o modelo específico deste trabalho, faremos uma degressão sobre a utilidade e a limitação de um modelo matemático, especialmente daqueles com envolvimento em biologia e dinâmica de populações.

Levins [14] abre a discussão definindo os três principais atributos de um modelo matemático: precisão, realismo e generalidade. Claramente, o ideal é trabalhar com um modelo que incorpore esses três atributos. No entanto, Levins argumenta que isso não é possível e que poderemos incorporar no máximo dois desses atributos. Por isso, a forma de modelar depende do problema trabalhado. Um biólogo interessado em prevenir grandes epidemias de cólera, por exemplo, pode sacrificar a generalidade, em troca de um modelo que lhe dé informações precisas e realistas acerca do comportamento da doença. Por outro lado um biólogo interessado em entender a estranha relação de presa-predador em corais (mais predadores do 
que presas), pode sacrificar precisão, em prol de obter informações sobre o mecanismo que impera nesses eco-sistemas.

Dessa forma, para saber qual atributo um modelo deve priorizar, o pesquisador deve saber qual pergunta ele está tentando responder. Note que isso é uma constante nos dois exemplos anteriores. Em um sistema para a interação de populações a pergunta gira em torno dos mecanismos que controlam a dinâmica. No modelo que trabalharemos, mais presa significa menos caça para o predador. A principal questão é saber que impactos isso pode trazer para um sistema biológico e descobrir os mecanismos que controlam a dinâmica qualitativa (e não quantitativa) desse tipo de ecossistema.

\subsubsection{Evolução dos modelos presa-predador}

O primeiro modelo de presa-predador foi desenvolvido por Volterra. Ele tinha a intenção de estudar o aumento na presença de "selachians", (tubarões, raias, etc) em portos italianos durante a primeira guerra mundial, quando a pesca teve uma grande diminuição. A partir de agora, chamaremos de $x$ a densidade de presa (peixes nesse caso) e de $y$ a densidade de predadores (tubarões nesse caso):

$$
\left\{\begin{array}{l}
\dot{x}=a x-m x y \\
\dot{y}=-\delta y+c x y
\end{array}\right.
$$

onde:

- $a$ é o crescimento intrínseco da população de presas;

- $\delta$ a taxa de mortalidade natural da população de predadores;

- $m>0$ a taxa de eficiência da caça;

- $c>0$ a taxa de eficiência de caça vezes a taxa de conversão da caça em biomassa de predadores (normalmente é esperado $m>c$ ).

Obviamente, o interesse biológico encontra-se nas áreas onde $x \geq 0$ e $y \geq 0$, como em qualquer modelo desse tipo. A análise é fácil e nos fornece um único equilíbrio no interior do 
domínio de interesse. Tal equilíbrio é sempre um centro.

Imaginar um crescimento exponencial da presa não é muito realista. Por isso, podemos imaginar um crescimento logístico, no qual o sistema passa a ser:

$$
\left\{\begin{array}{c}
\dot{x}=a x-\lambda x^{2}-m x y \\
\dot{y}=-\delta y+c x y
\end{array}\right.
$$

onde $\lambda$ é a taxa de competição entre as presas.

Se quisermos tornar o modelo mais realista ainda, podemos imaginar que a taxa de conversão de presa em predador não é, necessariamente, proporcional à quantidade de encontros entre elas (simples encontro vezes a eficacia da caça). Isso significaria que um predador tentaria consumir a presa sempre que a encontrasse. No entanto, um predador vai se saciar eventualmente, por isso, considerar a taxa de conversão como $x y$ é pouco realista.

Isso nos leva a introdução das funções resposta $P(x)$ :

$$
\left\{\begin{array}{c}
\dot{x}=a x-\lambda x^{2}-P(x) y \\
\dot{y}=-\delta y+c P(x) y
\end{array}\right.
$$

A função resposta $P(x)$ pode ser de diversos tipos. As mais utilizadas, foram propostas por C.S. Holling $[15,1]$. As quatro principais funções resposta são:

- Holling I: $P(x)=m x$;

- Holling II: $P(x)=\frac{m x}{\beta+x}$;

- Holling III: $P(x)=\frac{m x^{2}}{\beta^{2}+x^{2}}$;

- Holling IV: $P(x)=\frac{m x}{\alpha x^{2}+\beta x+1}$

A função Holling I já foi discutida. Já a Holling II é obtida quando consideramos que o predador necessita de uma quantidade considerável de tempo para capturar e consumir a presa. Esta função tem especial importância na literatura especializada por combinar realismo biológico com facilidade de análise matemática. Já a função Holling III é interessante pois existe um ponto $x_{0}$ tal que: 
- caso a densidade de presa seja menor do que $x_{0}$ então o predador dificilmente vai conseguir caça-la;

- caso a densidade de presa seja maior do que $x_{0}$, o predador aumenta a procura pela presa proporcionalmente ao aumento da densidade dela.

Dessa forma podemos modelar um ecossistema que tenha refúgios, como um coral por exemplo. Por fim, a função Holling IV é interessante pois ela apresenta uma densidade de presa ótima, onde o predador consegue converter o máximo possível de presas em alimento, mas, para densidades maiores, o predador tem dificuldades na caça. Isso pode ser ocasionado por uma estratégia de defesa em grupo. Como por exemplo regiões onde as plantas são tão densas que os herbívoros tem dificuldades em adentrar-se nas matas para come-las.

O sistema 1.2 com função resposta do tipo Holling IV foi recentemente estuda por Zhu [6]. Em um esforço para tornar o sistema ainda mais real, vamos considerar que os predadores também competem entre si. Dessa forma, devemos adicionar um termo à equação, de modo que, finalmente obtemos:

$$
\left\{\begin{array}{c}
\dot{x}=x\left(\bar{a}-\bar{\lambda} x-\frac{\bar{m} y}{\bar{\alpha} x^{2}+\bar{\beta} x+1}\right) \\
\dot{y}=y\left(-\bar{\delta}-\bar{\mu} y+\frac{\overline{\bar{m}} \bar{m} x}{\bar{\alpha} x^{2}+\bar{\beta} x+1}\right)
\end{array}\right.
$$

Este é o sistema que trabalharemos seguindo as seguintes referencias $[1,2,3,4]$. Note que, se $\bar{\mu}=0$ estamos nas condições do sistema estudado por Zhu.

\subsubsection{O modelo escolhido}

Escolhemos o sistema:

$$
\left\{\begin{array}{c}
\dot{x}=x\left(\bar{a}-\bar{\lambda} x-\frac{\bar{m} y}{\bar{\alpha} x^{2}+\bar{\beta} x+1}\right) \\
\dot{y}=y\left(-\bar{\delta}-\bar{\mu} y+\frac{\bar{c} \bar{m} x}{\bar{\alpha} x^{2}+\bar{\beta} x+1}\right)
\end{array}\right.
$$

que possui oito parâmetros:

- $\bar{a}>0$ é a taxa de crescimento da presa;

- $\bar{\lambda}>0$ a taxa de competição entre as presas;

- $\bar{\delta}>0$ a taxa de mortalidade do predador; 
- $\bar{\mu} \geq 0$ a taxa de competição entre os predadores;

- $\bar{m}>0$ a taxa de sucesso da caça;

- $\bar{c}>0$ a taxa de conversão da caça em presa;

- $\bar{\alpha} \geq 0$ e $\bar{\beta}>-2 \sqrt{\bar{\alpha}}$ são coeficientes da função resposta.

Fazendo uma mudança de coordenadas e reescalonando o tempo (ver capítulo 2) obtemos:

$$
\left\{\begin{aligned}
\dot{x}=x\left(1-\lambda x-\frac{y}{\alpha x^{2}+\beta x+1}\right) & =f_{1}(x, y, \alpha, \beta, \mu, \delta, \lambda) \\
\dot{y}=y\left(-\delta-\mu y+\frac{x}{\alpha x^{2}+\beta x+1}\right) & =f_{2}(x, y, \alpha, \beta, \mu, \delta, \lambda)
\end{aligned}\right.
$$

que possui só cinco parâmetros. Existem certas restrições sobre os parâmetros: $\alpha \geq 0$, $\beta>-2 \sqrt{\alpha}, \mu \geq 0, \delta>0$ e $\lambda>0$, as quais serão discutidas no capítulo 2 . Os cinco parâmetros serão organizados da seguinte forma:

\section{Definição 1.1.3.}

$$
\begin{gathered}
\Delta=\left\{(\delta, \lambda) \in \mathbb{R}^{2} ; \delta>0, \lambda>0\right\} \\
\mathcal{W}=\left\{(\alpha, \beta, \mu) \in \mathbb{R}^{3} ; \alpha \geq 0, \beta>-2 \sqrt{\alpha}, \mu \geq 0\right\} \\
\mathcal{W}_{1}=\left\{(\alpha, \mu, \beta) \in \mathbb{R}^{3} ;(\alpha, \beta, \mu) \in \mathcal{W}, \beta>0\right\}
\end{gathered}
$$

Isso significa que estudaremos os sistemas com parâmetros localizados em $\Delta \times \mathcal{W}$. Em alguns momentos, nos restringiremos a $\Delta \times \mathcal{W}_{1}$. Faremos o estudo do modelo como uma família a dois parâmetros $(\Delta)$ de sistemas tri-paramétricos $(\mathcal{W})$. Por fim, só interessa estudar o que ocorre no conjunto $Q=\left\{(x, y) \in \mathbb{R}^{2} ; x \geq 0, y \geq 0\right\}$, com especial interesse no seu interior.

O estudo aqui desenvolvido consiste em descrever o retrato de fase e as mudanças qualitativas que apresentam dependendo dos parâmetros. Para isso, faremos uma análise das bifurcações.

\subsection{Bifurcações}

Intuitivamente, uma bifurcação ocorre quando existe alguma mudança qualitativa no comportamento de um sistema dinâmico com a mudança de parâmetros. No caso de um sistema 
de equações diferenciais autônomo, queremos comparar o comportamento do espaço de fase para valores distintos dos parâmetros. Para estudar quando existem mudanças, devemos primeiro definir quando que dois campos de vetores são equivalentes. Para isso, devemos ter uma relação de equivalência (reflexiva, simétrica e transitiva) entre os campos.

Para ser uma relação de equivalência útil, queremos que dois campos sejam equivalentes caso possamos estabelecer uma correspondência entre a família de órbita de um campo com a do outro. Com esse objetivo, definimos:

Definição 1.2.1. Sejam $X_{1}, X_{2}$ campos vetoriais de classe $C^{k}(k \geq 1)$ definidos nos abertos de $\mathbb{R}^{2}, \Delta_{1}, \Delta_{2}$ respectivamente. Dizemos que $X_{1}$ é topologicamente equivalente a $X_{2}\left(C^{r}\right.$ equivalente com $r \leq k$ ) caso exista um homeomorfismo $h: \Delta_{1} \rightarrow \Delta_{2}$ (difeomorfismo $C^{r}$ ) que leva órbita de $X_{1}$ em órbita de $X_{2}$ preservando a orientação. Em outras palavras, seja $p \in \Delta_{1}$ e $\gamma_{1}(p)$ a órbita orientada passando por $p$; então $h\left(\gamma_{1}(p)\right)$ é a órbita orientada $\gamma_{2}(h(p))$ de $X_{2}$ passando por $h(p)$.

Esta será nossa relação de equivalência entre dois campos. Considere uma família de campos $X_{\alpha}$ que depende de um parâmetro $\alpha \in \mathbb{R}^{n}$. Variando o parâmetro, podemos alterar o re de fase do sistema. No entanto, ele pode continuar topologicamente equivalente ao primeiro campo ou não. Assim:

Definição 1.2.2. Uma bifurcação ocorre quando aparecem dois campos que não são topologicamente equivalentes ao variarmos os parâmetros. Em outras palavras, $\alpha_{0}$ é um parâmetro de bifurcação caso, para qualquer $\epsilon>0$ exista $\alpha_{1}$, $\epsilon$-próximo a $\alpha_{0}$ tal que $X_{\alpha_{1}}$ e $X_{\alpha_{0}}$ não sejam topologicamente equivalentes.

Vejamos um exemplo (talvez o mais simples) de bifurcação:

Exemplo 1.2.3. Tomemos o campo:

$$
\left\{\begin{array}{c}
\dot{x}=\alpha+x^{2} \\
\dot{y}=y
\end{array}\right.
$$

$\operatorname{com} \alpha \in \mathbb{R}$. 
Notemos que, para $\alpha<0$ temos dois equilíbrios, sendo um deles uma sela e o outro um nó. Dessa forma, não é difícil mostrar que dois campos com $\alpha<0$ são topologicamente equivalentes. Já para $\alpha>0$ não temos equilíbrios e também não é difícil mostrar que dois campos com $\alpha>0$ são topologicamente equivalentes. No entanto, um campo com $\alpha>0$ e outro com $\alpha<0$ não são topologicamente equivalentes. Logo $\alpha=0$ é um parâmetro de bifurcação.

Essa bifurcação recebe o nome de sela-nó.

Dentre as bifurcações, podemos dividi-las em locais e globais. Uma bifurcação local dos equilíbrios se caracteriza quando podemos percebe-la em qualquer vizinhança do equilíbrio que tomemos, não importa o quão pequena. Podemos também definir bifurcação local de órbitas periódicas, onde o conceito é o mesmo, mas devemos tomar uma vizinhança em alguma secção transversal da órbita periódica. As demais bifurcações serão chamadas de globais. O exemplo anterior constitui uma bifurcação local.

Nesta dissertação, vamos estudar as bifurcações locais dos equilíbrios do sistema 1.1 e não vamos nos preocupar com as bifurcações globais, onde o grau de dificuldade é maior.

Agora, tome um parâmetro $\alpha_{0}$ fixo. Existe um conjunto $A_{\alpha_{0}}$ maximal do espaço de parâmetros, tal que, para todo $\alpha_{1} \in A_{\alpha_{0}}, X_{\alpha_{1}}$ é topologicamente equivalente a $X_{\alpha_{0}}$. Chamamos esse conjunto de estrato de $\alpha_{0}$.

Definição 1.2.4. Um diagrama de bifurcação é a divisão do espaço de parâmetros em conjuntos de estratos, onde cada estrato é relacionado com um representante da configuração topológica do campo naquele estrato.

O diagrama de bifurcações que vamos discutir terá estratos ligeiramente diferentes do que definimos: pediremos que os campos sejam apenas localmente equivalentes.

Definição 1.2.5. A codimensão de uma bifurcação em um sistema dinâmico $X_{\alpha}$ é a diferença entre a dimensão do espaço de parâmetros e a dimensão do estrato que contém essa bifurcação. 
É possível classificar as bifurcações em certas classes. Assim:

Definição 1.2.6. Um sistema $X_{\alpha}$ é topologicamente equivalente a $Y_{\beta}$ se:

- Existe um homeomorfismo no espaço de parâmetros $p: \mathbb{R}^{m} \rightarrow \mathbb{R}^{m}, \beta=p(\alpha)$.

- Existe uma família de homeomorfismos $h_{\alpha}: \mathbb{R}^{n} \rightarrow \mathbb{R}^{n}$ tal que, para cada $\alpha_{0}$ fixado, $h_{\alpha_{0}}$ é a conjugação topológica entre $X_{\alpha_{0}}$ e $Y_{p\left(\alpha_{0}\right)}$.

Isso permite classificar as diferentes bifurcações que podem ocorrer. A teoria de bifurcações já possui uma classificação completa até codimensão três, pelo menos das bifurcações de equilíbrios. Tal classificação se baseia em três condições: condições necessárias, de não degenerescência e de transversalidade, que oportunamente serão discutidas em cada caso que se apresentam neste trabalho.

\subsubsection{Bifurcações encontradas no modelo}

Agora, vamos começar a tratar do sistema proposto. Comecemos tabelando as bifurcações de equilíbrios que ele apresenta:

\begin{tabular}{|l|c|r|}
\hline Notação & Nome & Subordinadas \\
\hline$T C_{1}$ & Transcrítica & \\
\hline$T C_{2}$ & Transcrítica degenerada & $S N_{1}+T C_{1}$ \\
\hline$T C_{3}$ & Transcrítica duplamente degenerada & $S N_{2}+T C_{2}$ \\
\hline$S N_{1}$ & Sela-nó (Dobra) & \\
\hline$S N_{2}$ & Sela-nó degenerada (Cúspide) & \\
\hline$H_{1}$ & Hopf & \\
\hline$H_{2}$ & Hopf degenerada (Bautin) & $H_{1}+S N_{1}$ \\
\hline$B T$ & Bogdanov-Takens & $B T+H_{2}$ \\
\hline$B T_{3}$ & Bogdanov-Takens degenerada & $B T+H_{2}+S N_{2}$ \\
\hline$N F_{3}$ & Foco nilpotente & $B T+H_{2}+S N_{2}$ \\
\hline$N E_{3}$ & Elíptica nilpotente & \\
\hline
\end{tabular}

Uma bifurcação $b$ está subordinada a uma bifurcação $a$ se, no diagrama de bifurcações, para cada ponto do tipo $a$ existe uma curva formada por $b$ que se aproxima arbitrariamente de $a$. 
Nesta tabela não estão relacionadas as bifurcações globais ou de órbitas periódicas subordinadas a essas bifurcações.

Para o estudo das bifurcações deste trabalho sugerimos: [8], [9] e [10]. Ao longo deste trabalho apresentaremos as propriedades mais importantes destas bifurcações.

Por fim, encontraremos no trabalho uma bifurcação que, até onde sabemos, ainda não foi estudada. Ela consiste em uma transição entre foco nilpotente e elíptica nilpotente. Em certas partes do trabalho, vamos nos referir a $N F_{3}$ e $N E_{3}$ simplesmente como bifurcações nilpotentes.

\subsection{Esquema dos resultados deste trabalho}

Neste trabalho vamos fazer um estudo das bifurcações locais em equilíbrios do sistema 1.1. Os principais resultados são:

Teorema 1.3.1. (Plano Transcrítico) Para todo $(\delta, \lambda) \in \Delta$ o diagrama de bifurcação do sistema em $\mathcal{W}$ associado ao ponto $C=\left(\frac{1}{\lambda}, 0\right)$ possui um ponto transcrítico de codimensão 3 $\left(T C_{3}\right)$, duas semi-retas conexas transcríticas de codimensão $2\left(T C_{2}\right)$ subordinadas a $T C_{3} e$ dois semi-planos conexos transcríticos de codimensão 1 (TC $\left.C_{1}\right)$ subordinadas a $T C_{2}$ e $T C_{3}$. $A$ união de $T C_{1}, T C_{2}$ e $T C_{3}$ é um plano em $\mathcal{W}$ que chamaremos de $T C$. Os conjuntos $T C_{1}$, $T C_{2}$ e $T C_{3}$ são não vazios para todos os $(\delta, \lambda) \in \Delta$. Não ocorrem mais degenerescências.

Teorema 1.3.2. (Divisão de $\Delta$ ) Existem três curvas que dividem $\Delta: \Omega_{1}, \Omega_{2}$ e $\Omega_{3}$, sendo que $\Omega_{3}$ está sempre abaixo de $\Omega_{2}$. Dessa forma, podemos dividir um conjunto aberto e denso de $\Delta$ em três componentes conexas, cada uma com um diagrama de bifurcação diferente:

- Acima da curva $\Omega_{1}$, não ocorrem bifurcações de Bogdanov-Takens de codimensão três.

- Abaixo da curva $\Omega_{1}$, ocorre pelo menos uma bifurcação de Bogdanov-Takens de codimensão três.

- Acima da curva $\Omega_{2}$, não ocorrem bifurcações nilpotentes. 

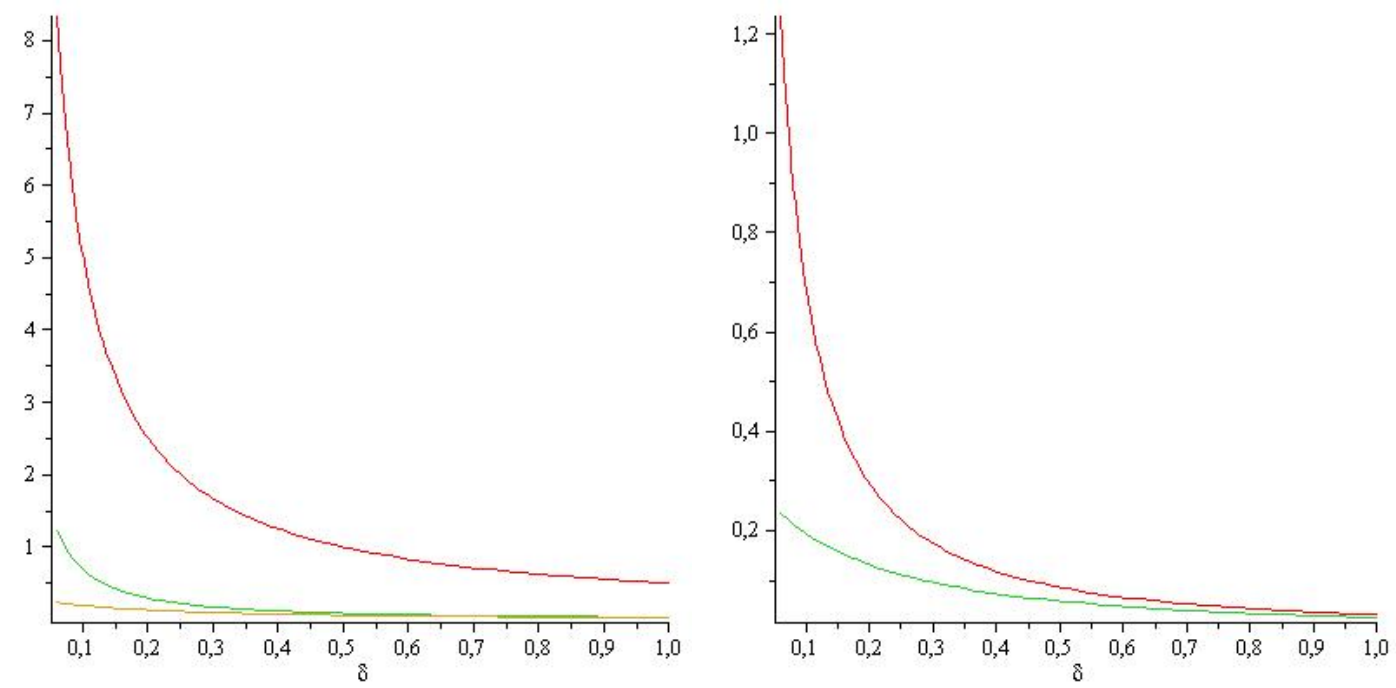

Figura 1.1: À esquerda: A curva acima (vermelho) é $\Omega_{1}$, a do meio (verde) é $\Omega_{2}$ e a de baixo (amarela) é $\Omega_{3}$. À direita: A curva acima (vermelho) é $\Omega_{2}$ e a de baixo (verde) é $\Omega_{3}$. Compare a curva $\Omega_{2}$ com a obtida em [1].

- Entre a curva $\Omega_{3}$ e $\Omega_{2}$, ocorrem pelo menos duas bifurcações nilpotentes, sendo que elas ocorrem para parâmetros válidos (pois $\alpha<0$ ).

- Abaixo da curva $\Omega_{3}$, ocorre pelo menos duas bifurcações nilpotentes, sendo que, pelo menos uma dessas bifurcações ocorre para parâmetros não válidos. Ver figura 1.1.

Com esses resultados e mais alguns obtidos de forma computacional, seremos capazes de desenhar o diagrama de bifurcação do sistema, pelo menos em termos qualitativos, como vemos na figura 1.2 .

Além disso, seremos capazes de demonstrar os seguintes resultados:

Teorema 1.3.3. (Dominio de captura) Para $(\alpha, \beta, \mu, \delta, \lambda) \in \mathcal{W} \times \Delta$ e para todo $p \in \mathbb{R}$ tal que:

$$
p>\frac{1}{\lambda}\left(\frac{1}{4 \delta}(1-\delta)^{2}+1\right)=f(\delta, \lambda)
$$

o domínio compacto $B_{p}=\{(x, y) \| x+y \leq p, x \geq 0, y \geq 0\}$ é invariante por $X_{t}$ com $t>0$, onde $X_{t}$ é o fluxo do campo $X=\left(f_{1}, f_{2}\right)$. 



Figura 1.2: À esquerda $(\delta, \lambda)$ abaixo da curva $\Omega_{2}$. À direita, $(\delta, \lambda)$ acima da curva $\Omega_{2}$. Em cada um dos planos que apresentamos, faremos um diagrama de bifurcação global do sistema, como vemos nas figuras 1.3 e 1.4 .

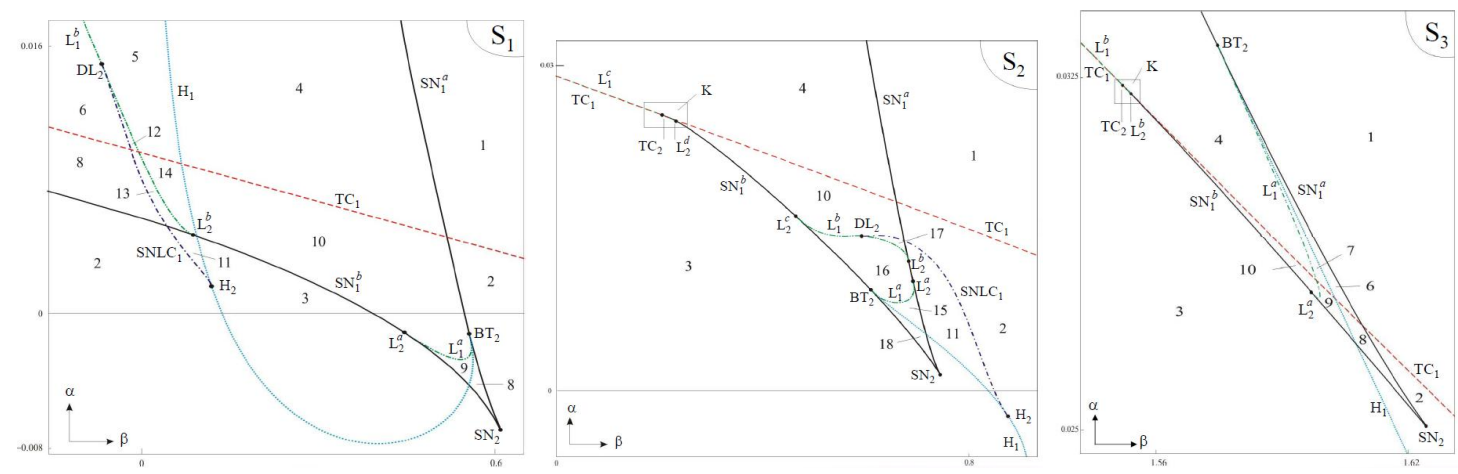

Figura 1.3: Planos $S 1, S 2$ e $S 3$.
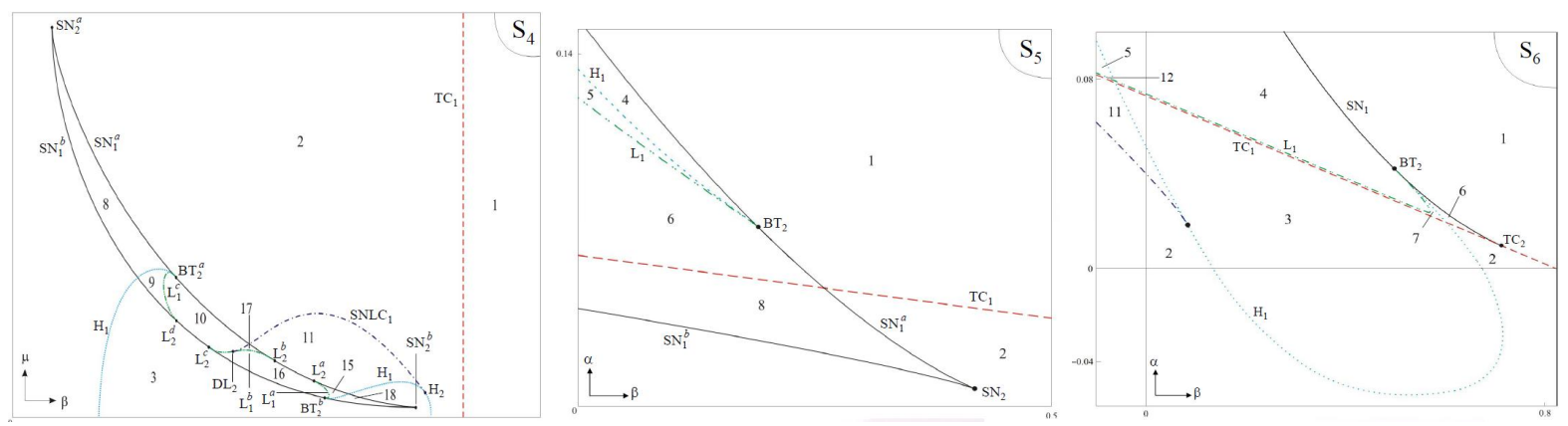

Figura 1.4: Planos $S 4, S 5$ e $S 6$. 

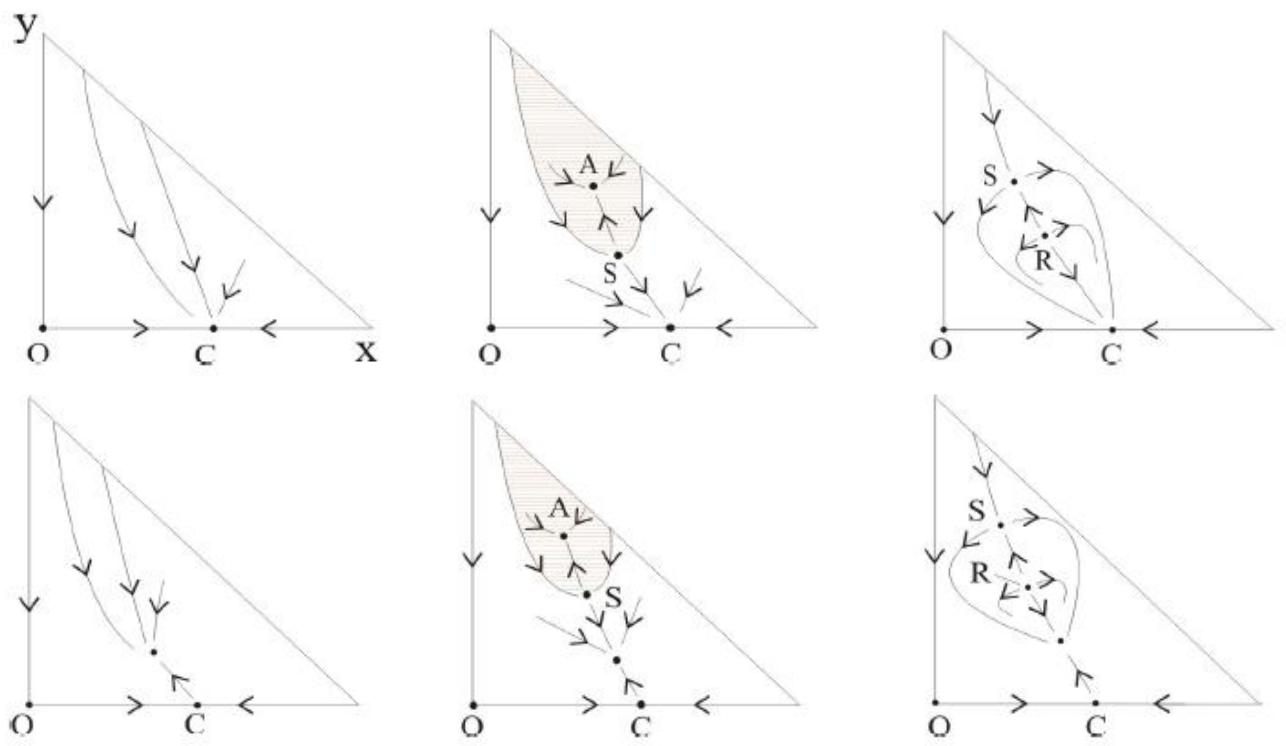

Figura 1.5: Acima: Possíveis configurações dos equilíbrios quando $C$ é um atrator. Abaixo: Possíveis configurações dos equilíbrios quando $C$ é uma sela. $A$ denota atratores, $R$ repulsores e $S$ selas. Figura reproduzada de [1].

Teorema 1.3.4. (Número de equilíbrios) Fixados $(\alpha, \beta, \mu, \delta, \lambda) \in \mathcal{W}_{1} \times \Delta$ com $\delta \lambda>\frac{1}{8}$, o sistema 1.1 tem dois equilíbrios nos eixos (origem e $C$ ) e pode ter zero, um, dois ou três equilíbrios no interior do quadrante $Q=\left\{(x, y) \in \mathbb{R}^{2} ; x \geq 0, y \geq 0\right\}$.

Além de demonstrar estes teoremas, citaremos resultados encontrados por outros autores, de forma completar a análise do modelo. O principal resultado nesse sentido é:

Proposição 1.3.5. (Classificação sem ciclos-limites) A menos dos ciclos-limites, se $\left(f_{1}, f_{2}\right)$ for genérico (ver capítulo 8 para um definições) então ele tem seis possibilidades de espaços de fase, listados na figura: 1.5 .

\subsubsection{Organização do trabalho}

Os resultados deste trabalho são divididos em sete capítulos. No capítulo 2 abrimos a discussão do modelo com resultados básicos: a mudança de coordenadas do sistema e a prova do teorema sobre o domínio de captura. No capítulo 3 analisamos os equilíbrios que se encontram no bordo de $Q$ e suas bifurcações. Neste caso trataremos da bifurcação transcrítica. 
Este capítulo contém a análise da forma normal desta bifurcação e os estratos de bifurcação.

Em seguida começamos a tratar as bifurcações no interior do quadrante positivo $Q$. Nos capítulos 4 e 5 tratamos das condições analíticas que caracterizam as bifurcações do tipo sela-nó e Hopf respectivamente. No capítulo 6 fazemos um estudo preparatório para o capítulo 7. Além disso, fixaremos o parâmetro $\beta$ como positivo e obteremos algumas informações geométricas e analíticas sobre as superfícies de bifurcação do tipo sela-nó, Hopf e sobre os equilíbrios hiperbólicos do sistema.

No capítulo 7 trataremos das bifurcações de Bogdanov-Takens e suas degenerescências. Apresentaremos o cálculo das formas normais do foco-nilpotente e da bifurcação de BogdanovTakens de codimensão três. Aplicaremos esses resultados sobre o sistema 1.1 e vamos analisar a validade dos parâmetros desses pontos. No capítulo 8 fecharemos o trabalho com o teorema acerca do número de equilíbrios e uma conjectura acerca do diagrama de bifurcação do sistema. 


\section{Capítulo 2}

\section{Estudo inicial do sistema}

\subsection{Simplificando o sistema}

Começamos o estudo com o sistema com oito parâmetros:

$$
\left\{\begin{array}{c}
\dot{x}=\bar{a} x-\bar{\lambda} x^{2}-\frac{\bar{m} x y}{\bar{\alpha} x^{2}+\bar{\beta} x+1} \\
\dot{y}=y\left(-\bar{\delta}-\bar{\mu} y+\frac{\bar{c} \bar{m} x}{\bar{\alpha} x^{2}+\bar{\beta} x+1}\right)
\end{array}\right.
$$

nos quais:

$$
\begin{array}{cccc}
\bar{a}>0 & \bar{m}>0 & \bar{c}>0 & \bar{\alpha} \geq 0 \\
\bar{\beta}>-2 \sqrt{\bar{\alpha}} & \bar{\mu} \geq 0 & \bar{\delta}>0 & \bar{\lambda}>0
\end{array}
$$

Cada um desses parâmetros tem um interesse biológico. No entanto, do ponto de vista matemático, cada parâmetro a mais, impõe uma dificuldade maior. Assim, vamos fazer uma mudança de variáveis de forma a facilitar o estudo de nosso sistema. Tomaremos:

$$
\begin{aligned}
& z(t)=\frac{\bar{c} \bar{m}}{\bar{a}} x\left(\frac{t}{\bar{a}}\right) \\
& w(t)=\frac{\bar{m}}{\bar{a}} y\left(\frac{t}{\bar{a}}\right)
\end{aligned}
$$

Obtendo:

$$
\begin{gathered}
\dot{z}=\frac{\bar{c} \bar{m}}{\bar{a}^{2}}\left(\bar{a} x-\bar{\lambda} x^{2}-\frac{\bar{m} x y}{\bar{\alpha} x^{2}+\bar{\beta} x+1}\right) \\
\dot{w}=\frac{\bar{m}}{\bar{a}^{2}}\left(y\left(-\bar{\delta}-\bar{\mu} y+\frac{\bar{c} \bar{m} x}{\bar{\alpha} x^{2}+\bar{\beta} x+1}\right)\right)
\end{gathered}
$$

E, tomando:

$$
\alpha=\frac{\bar{a}^{2}}{\bar{m}^{2} \bar{c}^{2}} \bar{\alpha} \quad \beta=\frac{\bar{a}}{\bar{m} \bar{c}} \bar{\beta} \quad \mu=\frac{1}{\bar{m}} \bar{\mu} \quad \delta=\frac{1}{\bar{a}} \bar{\delta} \quad \lambda=\frac{1}{\bar{c} \bar{m}} \bar{\lambda}
$$


temos:

$$
\left\{\begin{array}{c}
\dot{z}=z\left(1-\lambda z-\frac{w}{\alpha z^{2}+\beta z+1}\right) \\
\dot{w}=w\left(-\delta-\mu w+\frac{z}{\alpha z^{2}+\beta z+1}\right)
\end{array}\right.
$$

Substituindo $z$ por $x$ e $w$ por $y$ temos:

$$
\left\{\begin{array}{c}
\dot{x}=x\left(1-\lambda x-\frac{y}{\alpha x^{2}+\beta x+1}\right) \\
\dot{y}=y\left(-\delta-\mu y+\frac{x}{\alpha x^{2}+\beta x+1}\right)
\end{array} 1.1\right.
$$

com:

$$
\alpha \geq 0 \quad \beta>-2 \sqrt{\alpha} \quad \mu \geq 0 \quad \delta>0 \quad \lambda>0
$$

Lembremos que o conjunto de parâmetros admissíveis é $\mathcal{W} \times \Delta$ onde:

$$
\begin{gathered}
\mathcal{W}=\{(\alpha, \beta, \mu) \| \alpha \geq 0, \beta>-2 \sqrt{\alpha}, \mu \geq 0\} \\
\Delta=\{(\delta, \lambda) \| \delta>0, \lambda>0\}
\end{gathered}
$$

\subsection{Breve estudo sobre a função resposta}

Em modelos de presa predador normalmente existe uma função resposta. Neste sistema, estamos interessados em estudar a função resposta do tipo Holling IV. Chamaremos tal função de $P(x, \alpha, \beta)$ :

$$
\begin{aligned}
& P: \mathbb{R}_{+} \times \mathcal{W}_{\mu} \rightarrow \mathbb{R} \\
& (x, \alpha, \beta) \mapsto \frac{x}{\alpha x^{2}+\beta x+1}
\end{aligned}
$$

onde

$$
\mathcal{W}_{\mu}=\left\{(\alpha, \beta) \in \mathbb{R}^{2} \| \alpha>0, \beta>-2 \sqrt{\alpha}\right\}
$$

Por abuso de notação, denotaremos $P(x, \alpha, \beta)$ simplesmente por $P(x)$.

Vamos começar entendendo um pouco sobre as propriedades dessa função. A primeira coisa é mostrar que a função está de fato bem definida. Assim sendo, temos que mostrar que $\alpha x^{2}+\beta x+1 \neq 0$ para $x \geq 0$. Assim, notemos que as raízes de $\alpha x^{2}+\beta x+1$ caso $\alpha \neq 0$ são:

$$
\begin{aligned}
& x_{1}=-\frac{\beta}{2 \alpha}+\frac{\sqrt{\beta^{2}-4 \alpha}}{2 \alpha} \\
& x_{2}=-\frac{\beta}{2 \alpha}-\frac{\sqrt{\beta^{2}-4 \alpha}}{2 \alpha}
\end{aligned}
$$


Assim, caso $\beta^{2}-4 \alpha<0$, teremos duas raízes complexas e portanto o polinômio não zera. Isso equivale a $-2 \sqrt{\alpha}<\beta<2 \sqrt{\alpha}$. Como nos restringimos a $\beta>-2 \sqrt{\alpha}$, resta analisar quando $0<2 \sqrt{\alpha} \leq \beta$. Neste caso, é fácil ver que $x_{1}$ e $x_{2}$ são menores que zero.

Resta agora o caso em que $\alpha=0$. Neste caso a raiz de $\beta x+1$ é:

$$
\bar{x}=-\frac{1}{\beta}
$$

Agora, como $\beta>-2 \sqrt{\alpha}=0$, temos que $\bar{x}<0$. Assim sendo, $P(x)$ está bem definida para todo $x \geq 0$. Assim, vale notar que o polinômio $\alpha x^{2}+\beta x+1$ é sempre positivo, uma vez que não possui raízes em $x \geq 0$ e $P(0)=1$. Este fato será usado sem maiores preocupações ao longo do trabalho.

Agora, vamos estudar um pouco o comportamento de $P(x)$. Para isso, note que a derivada de $P(x)$ vale:

$$
P^{\prime}(x)=\frac{1-\alpha x^{2}}{\left(\alpha x^{2}+\beta x+1\right)^{2}}
$$

Agora, vamos dividir em dois casos:

- $\alpha=0$

- $\alpha \neq 0$

No primeiro caso, a derivada nunca se anula e o limite de $P(x)$ quando $x$ tende a infinito é igual a:

$$
\lim _{x \rightarrow \infty} P(x)=\frac{1}{\beta}
$$

Já no segundo caso, temos que $P^{\prime}(x)$ se anula no ponto $\bar{x}=\frac{1}{\sqrt{\alpha}}$. Este ponto constitui um ponto de máximo da função $P(x)$ e assim temos que:

$$
\begin{gathered}
\lim _{x \rightarrow \infty} P(x)=0 \\
\max _{x \geq 0} P(x)=\frac{1}{\beta+2 \sqrt{\alpha}}
\end{gathered}
$$

Assim, caso $\alpha \neq 0$, essa função resposta é tal que, quando existe uma quantidade grande de presas, os predadores tem dificuldade em caça-las. Tal função resposta é boa para estudar a dinâmica presa-predador na qual a presa tenha algum mecanismo de defesa em grupo. Como no caso de morsas e ursos polares. 


\subsection{Domínio de captura}

Nesta secção, nosso interesse é mostrar que, sempre que $(\delta, \lambda) \in \Delta$ estiver fixado, o estudo dos equilíbrios do sistema 1.1 pode se restringir a um domínio compacto de $Q=\{(x, y) \in$ $\left.\mathbb{R}^{2} ; x \geq 0, y \geq 0\right\}$.

Teorema 2.3.1. (Dominio de captura) Para $(\alpha, \beta, \mu, \delta, \lambda) \in \mathcal{W} \times \Delta$ e para todo $p \in \mathbb{R}$ tal que:

$$
p>\frac{1}{\lambda}\left(\frac{1}{4 \delta}(1-\delta)^{2}+1\right)=f(\delta, \lambda)
$$

o domínio compacto $B_{p}=\{(x, y) \| x+y \leq p, x \geq 0, y \geq 0\}$ é invariante por $X_{t}$ com $t>0$, onde $X_{t}$ é o fluxo do campo $X=\left(f_{1}, f_{2}\right)$. 1.3.3

Demonstração. Comecemos estudando as singularidades no bordo de $Q:(0,0)$ e $C=\left(\frac{1}{\lambda}, 0\right)$.

Afirmo que:

- $\partial Q$ é invariante;

- não existem outros equilíbrios em $\partial Q$;

- $(0,0)$ e $C=\left(\frac{1}{\lambda}, 0\right)$ são os $\omega$-limite das órbitas contidas em $\partial Q$.

De fato, sobre o eixo $x$, temos que $\dot{y}=0$ e $\dot{x}=x-\lambda x^{2}$. Quando igualamos a zero $\dot{x}$ temos que as únicos equilíbrios são a origem e $C$. Além disso, é fácil ver que, se $x>0$ então a órbita que passa por $(x, 0)$ tem como $\omega$-limite $C$. Já sobre o eixo $y$, temos que $\dot{x}=0$ e que $\dot{y}=-\delta y-\mu y^{2}$. Quando igualamos a zero $\dot{y}$ temos dois equilíbrios: $y=0$ e $y=-\frac{\delta}{\mu}$. No entanto apenas a primeira pertence a $\partial Q$. Por fim, é fácil ver que se $y>0$ então a órbita que passa por $(0, y)$ tem como $\omega$-limite a origem.

Agora, vamos estudar a reta $x+y=p$. Queremos achar $p$ tal que $\{(x, y) \| x \geq 0, y \geq$ $0, x+y \leq p\}$ seja invariante por $X_{t}$. Comecemos notando que a reta $x+y=p$ tem como vetor normal $(1,1)$. Assim, se $q \in B_{p}$ e $\phi(t, q)$ é a órbita de $X$ tal que $\phi(0, q)=q$, então, para que $\phi(t, q)$ permaneça em $B_{p}$ para todo $t \geq 0$ devemos ter:

- $p>\frac{1}{\lambda}$ (Condição sobre o bordo) 
- $\langle(1,1),(\dot{x}, \dot{y})\rangle$ tenha sinal negativo ao longo de todo segmento de reta $x+y=p$ com $x>0$ e $y>0$. (Condição sobre o segmento de reta $x+y=p$ )

Assim:

$$
\langle(1,1),(\dot{x}, \dot{y})\rangle=x(1-\lambda x)+y(-\delta-\mu y)=g(x, y, \mu, \delta, \lambda)
$$

Queremos que $g(x, y, \mu, \delta, \lambda)<0$ ao longo do segmento de reta. Para tal, comecemos notando que:

$$
\begin{gathered}
g(0, p, \mu, \delta, \lambda)=-\delta p-\mu p^{2}<0 \\
g(p, 0, \mu, \delta, \lambda)=p-\lambda p^{2}<0 \text { caso } p>\frac{1}{\lambda}
\end{gathered}
$$

Assim, se $p>\frac{1}{\lambda}$, os extremos são negativos. Portanto, se houver alteração de sinal, este deve ocorrer no segmento. Considere agora $h(x, y, \mu, \delta, \lambda)=x(1-\lambda x)-\delta y$. Note que:

$$
\begin{gathered}
h(x, y, \mu, \delta, \lambda)<0 \Rightarrow g(x, y, \mu, \delta, \lambda)<0 \\
h(0, p, \mu, \delta, \lambda)<0 \\
h(p, 0, \mu, \delta, \lambda)<0
\end{gathered}
$$

Ao contrário de $g(x, y, \mu, \delta, \lambda), h(x, y, \mu, \delta, \lambda)$ é linear em $y$. Assim, vamos analisar o sinal de $h(x, y, \mu, \delta, \lambda)$ por simplicidade. Quando este for negativo, o sinal de $g(x, y, \mu, \delta, \lambda)$ também o será.

Da mesma forma que $g$, se $h$ mudar de sinal, deve faze-lo fora dos extremos. Assim, pela continuidade de $h$ haverá um ponto de máximo $(\bar{x}, \bar{y})$ tal que $\bar{x}>0, \bar{y}>0$ e $\bar{x}+\bar{y}=p$. Além disso, nesse ponto, $D h_{(\bar{x}, \bar{y}, \mu, \delta, \lambda)}$ e $(1,1)$ devem ser LD. Assim:

$$
\left|\begin{array}{cc}
1-\lambda \bar{x} & -\delta \\
1 & 1
\end{array}\right|=0
$$

o que implica que $1-\lambda \bar{x}+\delta=0$. Chegamos então que:

$$
\begin{gathered}
\bar{x}=\frac{1+\delta}{\lambda} \\
\bar{y}=p-\frac{1+\delta}{\lambda}
\end{gathered}
$$

Agora, $h(\bar{x}, \bar{y}, \mu, \delta, \lambda)=\frac{1}{4 \lambda}(1-\delta)^{2}+\frac{\delta}{\lambda}(1-p \lambda)$. Assim sendo, se $p>\frac{1}{\lambda}\left(\frac{1}{4 \delta}(1-\delta)^{2}+1\right)=f(\delta, \lambda)$ então $h(\bar{x}, \bar{y}, \mu, \delta, \lambda)<0$ o que implica que $h(x, y, \mu, \delta, \lambda)<0$ e portanto $g(x, y, \mu, \delta, \lambda)<0$ para $(x, y) \in\{x+y=p, x \geq 0, y \geq 0\}$. Note que, como $\delta>0$ então $f(\delta, \lambda)>\frac{1}{\lambda}$. Assim, caso $p>f(\delta, \lambda)$ então as duas condições são satisfeitas e portanto $B_{p}$ é invariante por $X_{t}$ para $t>0$. 


\subsection{Estudo da posição dos equilíbrios}

Temos como principal objetivo procurar as bifurcações dos equilíbrios do sistema 1.1. Para isso, devemos começar procurando os equilíbrios desse sistema. Notemos que, para que $(x, y)$ com parâmetros $(\alpha, \beta, \mu, \delta, \lambda)$ seja um equilíbrio no interior do quadrante positivo, devemos ter:

$$
\begin{gathered}
y=-(-1+\lambda x)\left(\alpha x^{2}+\beta x+1\right)=y_{x} \\
0=-\delta\left(\alpha x^{2}+\beta x+1\right)+\mu(-1+\lambda x)\left(\alpha x^{2}+\beta x+1\right)^{2}+x= \\
=F(x, \alpha, \beta, \mu, \delta, \lambda)
\end{gathered}
$$

Assim, procurar os equilíbrios do sistema corresponde a procurar as raízes de $F(x, \alpha, \beta, \mu, \delta, \lambda)$ para $(\alpha, \beta, \mu, \delta, \lambda)$ fixados. No entanto, não é possível resolver $F(x, \alpha, \beta, \mu, \delta, \lambda)=0$ por radicais em $x$. Dessa forma, utilizaremos essa expressão de forma implícita quando formos tratar das bifurcações. Por exemplo, quando $F(x, \alpha, \beta, \mu, \delta, \lambda)=0$ e $F_{x}(x, \alpha, \beta, \mu, \delta, \lambda)=0$ simultaneamente, temos uma dobra nos equilíbrios e, consequentemente, um candidato a bifurcação do tipo sela-nó.

Para não carregar a notação, vamos denotar a função $F(x, \alpha, \beta, \mu, \delta, \lambda)$ por $F(x)$ simplesmente. 


\section{Capítulo 3}

\section{Estudo da bifurcação transcrítica de C}

Estamos trabalhando com o sistema:

$$
\left\{\begin{array}{c}
\dot{x}=x-\lambda x^{2}-\frac{x y}{\alpha x^{2}+\beta x+1} \\
\dot{y}=y\left(-\delta-\mu y+\frac{x}{\alpha x^{2}+\beta x+1}\right)
\end{array}\right.
$$

onde os parâmetros $(\alpha, \beta, \mu, \delta, \lambda) \in \mathcal{W} \times \Delta$, onde $\mathcal{W}=\{(\alpha, \beta, \mu) ; \alpha \geq 0, \beta>-2 \sqrt{\alpha}, \mu \geq 0\}$ e $\Delta=\{(\delta, \lambda) ; \delta>0, \lambda>0\}$. Neste capítulo analisaremos as bifurcações que ocorrem sobre o bordo de $Q=\left\{(x, y) \in \mathbb{R}^{2} \mid x \geq 0, y \geq 0\right\}$. Isso se reduz a estudar as bifurcações que ocorrem no equilíbrio $C=\left(\frac{1}{\lambda}, 0\right)$.

De fato, só existem dois equilíbrios no bordo de $Q:(0,0)$ e $C=\left(\frac{1}{\lambda}, 0\right)$. No entanto, a origem é sempre hiperbólica já que a linearização do sistema calculada nela vale:

$$
\left[\begin{array}{cc}
1 & 0 \\
0 & -\delta
\end{array}\right]
$$

ela é, portanto, uma sela.

Assim, vamos estudar as bifurcações que ocorrem em $C=\left(\frac{1}{\lambda}, 0\right)$. Neste ponto, ocorrerá uma bifurcação que chamaremos de trascrítica.

Definição 3.0.1. Seja $X_{\alpha}: \Omega \rightarrow \mathbb{R}^{2}$ uma família de campos definidas em um aberto $\Omega \subseteq \mathbb{R}^{2}$ que dependem de um parâmetro $\alpha \in \mathbb{R}^{n}$. Suponha que exista uma curva $\gamma \subset \Omega$ invariante 
pelo campo para qualquer parâmetro. Suponha que exista um equilíbrio do campo, $p(\alpha) \in \gamma$, tal que $p(\alpha)$ é sempre hiperbólico no campo restrito a $\gamma$ e é genericamente hiperbólico no campo sobre $\Omega$. Diremos que ocorre uma bifurcação transcrítica quando o ponto $p(\alpha)$ deixa de ser hiperbólico em $\Omega$.

Dividiremos esse capítulo em quatro seç̧ões. Na primeira secção vamos achar candidatos a bifurcação transcríticas e vamos provar que elas satisfazem as condições de degenerescência e transversalidade. Na segunda secção vamos colocar o sistema na forma normal de um ponto transcrítico, de forma a estudarmos a sua dinâmica. Na terceira secção vamos procurar caracterizar o comportamento topológico do ponto $C$ dependendo dos parâmetros (uma diagrama de bifurcação local encontra-se na figura 3.1). Por fim, na última secção, vamos tratar da validade dos parâmetros caso fixemos $\beta$ positivo.

\subsection{A bifurcação transcrítica}

\subsubsection{Estratégia}

Estamos interessados em analisar as bifurcações do ponto de equilíbrio $C=\left(\frac{1}{\lambda}, 0\right)$. Afirmamos que ocorre uma bifurcação transcrítica de, no máximo, codimensão três. Intuitivamente, a bifurcação transcrítica ocorre quando um equilíbrio fixo do sistema colide com um outro equilíbrio. Caso o choque se dê com dois desses equilíbrios então teremos uma bifurcação de codimensão dois e assim por diante.

Assim, nossa estratégia é achar uma equação que nos forneça a multiplicidade do equilíbrio C. Para fazer isso, começaremos fazendo uma mudança de variáveis de forma que $C$ passe a estar na origem. Então, acharemos uma equação $G(x)$ cujos zeros são equilíbrios de nosso sistema. Assim, obteremos coeficientes que ao se anular degeneram a bifurcação. Então, analisaremos a conexidade e o formato geométrico de nossas bifurcações. Por fim, faremos uma análise das condições de transversalidade e de degenerescência. 


\subsubsection{A função $G(x)$}

Comecemos fazendo uma mudança de variáveis:

$$
\begin{gathered}
x=u+\frac{1}{\lambda} \\
y=v
\end{gathered}
$$

Nessas novas coordenadas, o sistema passa a ser:

$$
\left\{\begin{array}{c}
\dot{u}=-u-\lambda u^{2}-\frac{\lambda v(1+\lambda u)}{\lambda^{2}+\beta \lambda(1+\lambda u)+\alpha(1+\lambda u)^{2}}=U(u, v) \\
\dot{v}=v\left(-\delta-\mu v+\frac{\lambda(1+\lambda u)}{\lambda^{2}+\beta \lambda(1+\lambda u)+\alpha(1+\lambda u)^{2}}\right)=V(u, v)
\end{array}\right.
$$

Note que o ponto $C=\left(\frac{1}{\lambda}, 0\right)$ passa a ser a origem em nosso novo sistema. Assim, vamos calcular a parte linear do novo sistema na origem:

$$
A=\left[\begin{array}{rr}
-1 & \frac{\lambda}{\alpha+\lambda(\beta+\lambda)} \\
0 & -\delta+\frac{\lambda}{\alpha+\lambda(\beta+\lambda)}
\end{array}\right]
$$

A origem é hiperbólica caso $-\delta+\frac{\lambda}{\alpha+\lambda(\beta+\lambda)} \neq 0$. Definimos $T C$ como sendo o conjunto onde esse equilíbrio deixa de ser hiperbólico, ou seja, quando $-\delta+\frac{\lambda}{\alpha+\lambda(\beta+\lambda)}=0$. As bifurcações de $C$ ocorrerão neste conjunto. Note que para $U(u, v)$ se anular, $v$ deve ser igual a:

$$
V(u, \alpha, \beta, \mu, \delta, \lambda)=-\frac{u\left(\alpha u^{2} \lambda^{2}+2 \alpha u \lambda+\alpha+\beta \lambda^{2} u+\beta \lambda+\lambda^{2}\right)}{\lambda}
$$

Agora, substituindo $v$ por $V$ na equação da derivada de $v$ obtemos:

$$
\begin{gathered}
G(u, \alpha, \beta, \mu, \delta, \lambda)=-\alpha^{2} \lambda^{2} \mu u^{6}-\frac{\left(2 \alpha \lambda^{4} \mu \beta+4 \alpha^{2} \lambda^{3} \mu\right) u^{5}}{\lambda^{2}}-\frac{\left(6 \alpha^{2} \lambda^{2} \mu+\beta^{2} \lambda^{4} \mu+2 \alpha \lambda^{4} \mu+6 \alpha \lambda^{3} \mu \beta\right) u^{4}}{\lambda^{2}} \\
-\frac{\left(2 \beta^{2} \lambda^{3} \mu+4 \alpha \lambda^{3} \mu+4 \alpha^{2} \lambda \mu-\alpha \lambda^{3} \delta+2 \beta \lambda^{4} \mu+6 \alpha \lambda^{2} \mu \beta\right) u^{3}}{\lambda^{2}} \\
-\frac{\left(\mu \alpha^{2}+\lambda^{3}+2 \beta \lambda^{3} \mu+\beta^{2} \lambda^{2} \mu+\lambda^{4} \mu+2 \alpha \mu \lambda^{2}+2 \alpha \mu \beta \lambda-2 \alpha \lambda^{2} \delta-\beta \lambda^{3} \delta\right) u^{2}}{\lambda^{2}}-\frac{\left(-\lambda^{3} \delta-\beta \lambda^{2} \delta-\alpha \delta \lambda+\lambda^{2}\right) u}{\lambda^{2}}
\end{gathered}
$$

Por um abuso de notação, vamos nos referir a essa função apenas como $G(u)$. Note que $u=0$ satisfaz essa equação.

Estamos interessados em verificar a multiplicidade do equilíbrio (0,0), o que equivale a verificar a multiplicidade da raiz $u_{0}=0 \mathrm{em} G(u)$. Assim, vamos expandir $G(u)$ em torno de $u=0$ e obter:

$$
G(u)=\Lambda_{0} u+\Lambda_{1} u^{2}+\Lambda_{2} u^{3}+\Lambda_{3} u^{4}+\mathcal{O}\left(u^{5}\right)
$$


onde:

$$
\begin{gathered}
\Lambda_{0}=\frac{\delta \alpha+\delta \beta \lambda+\delta \lambda^{2}-\lambda}{\lambda} \\
\Lambda_{1}=-\frac{\mu \alpha^{2}+\lambda^{4} \mu+2 \beta \lambda^{3} \mu+\beta^{2} \lambda^{2} \mu+\lambda^{3}-\beta l^{3} \delta-2 \alpha \lambda^{2} \delta+2 \alpha \mu \beta \lambda+2 \alpha \mu \lambda^{2}}{\lambda^{2}} \\
\Lambda_{2}=-\frac{36 \alpha \mu \beta \lambda+24 \mu \alpha^{2}+12 \beta^{2} \lambda^{2} \mu-6 \alpha \lambda^{2} \delta+12 \beta \lambda^{3} \mu+24 \alpha \mu \lambda^{2}}{\lambda} \\
\Lambda_{3}=-24 \mu\left(6 \alpha \lambda \beta+\beta^{2} \lambda^{2}+6 \alpha^{2}+2 \alpha \lambda^{2}\right)
\end{gathered}
$$

Note que $\Lambda_{0}$ não é igual ao autovalor do linearizado do sistema. No entanto, não é difícil verificar que $\Lambda_{0}$ se anula se, e somente se, o autovalor $-\delta+\frac{\lambda}{\alpha+\lambda(\beta+\lambda)}$ também se anula. Assim, nossos termos nos fornecem os pontos onde ocorrem as bifurcações transcríticas, ainda que não sejam exatamente iguais a expansão do sistema sobre a variedade central.

Antes de começar as próximas análises, vamos definir algumas notações. Para $\left(\delta_{0}, \lambda_{0}\right) \in \Delta$ fixos, chamaremos de:

- $T C_{1}=\left\{(\alpha, \beta, \mu) \in \mathcal{W} ; \Lambda_{0}\left(\alpha, \beta, \mu, \delta_{0}, \lambda_{0}\right)=0, \Lambda_{1}\left(\alpha, \beta, \mu, \delta_{0}, \lambda_{0}\right) \neq 0\right\}$,

- $T C_{2}=\left\{(\alpha, \beta, \mu) \in \mathcal{W} ; \Lambda_{0}\left(\alpha, \beta, \mu, \delta_{0}, \lambda_{0}\right)=0, \Lambda_{1}\left(\alpha, \beta, \mu, \delta_{0}, \lambda_{0}\right)=0, \Lambda_{2}\left(\alpha, \beta, \mu, \delta_{0}, \lambda_{0}\right) \neq\right.$ $0\}$,

- $T C_{3}=\left\{(\alpha, \beta, \mu) \in \mathcal{W} ; \Lambda_{0}\left(\alpha, \beta, \mu, \delta_{0}, \lambda_{0}\right)=0, \Lambda_{1}\left(\alpha, \beta, \mu, \delta_{0}, \lambda_{0}\right)=0, \Lambda_{2}\left(\alpha, \beta, \mu, \delta_{0}, \lambda_{0}\right)=\right.$ $\left.0, \Lambda_{3}\left(\alpha, \beta, \mu, \delta_{0}, \lambda_{0}\right) \neq 0\right\}$,

Por fim, é interessante notar que o conjunto $T C$ que já havíamos definido é igual a:

$$
\left\{(\alpha, \beta, \mu) \in \mathcal{W} ; \Lambda_{0}\left(\alpha, \beta, \mu, \delta_{0}, \lambda_{0}\right)=0\right\}
$$

\subsubsection{Estudo da condição de não-degenerescência}

Queremos mostrar nesta secção que não ocorrem bifurcações transcríticas de multiplicidade maior do que três. Para isso, devemos mostrar que $\left(\Lambda_{0}, \Lambda_{1}, \Lambda_{2}, \Lambda_{3}\right) \neq(0,0,0,0)$ para todos os parâmetros admissíveis. De fato, consideremos por absurdo que $\left(\Lambda_{0}, \Lambda_{1}, \Lambda_{2}, \Lambda_{3}\right)=(0,0,0,0)$. Então para $\Lambda_{3}=0$ temos dois casos a considerar:

- $\mu=0$. Nesse caso, $\Lambda_{2}=0$ implica $\alpha=0$. Agora, como $\Lambda_{1}=0$ então $\beta=\frac{1}{\delta}$. Por fim, como $\Lambda_{0}=0$, então $\lambda=0$. Absurdo! 
- $6 \alpha^{2}+\beta^{2} \lambda^{2}+2 \alpha \lambda(3 \beta+\lambda)=0$. Neste caso, temos que: $6 \alpha^{2}+\beta^{2} \lambda^{2}+2 \alpha \lambda(3 \beta+\lambda)=$ $2 \alpha(\alpha+\lambda(\beta+\lambda))+(2 \alpha+\lambda \beta)^{2}$. Agora, como $\Lambda_{0}=0$, temos que $(\alpha+\lambda(\beta+\lambda))=\frac{\lambda}{\delta}$ que é maior que zero. Assim, devemos ter $\alpha=0$ e $\beta=0$. Mas $\beta>-2 \sqrt{\alpha}=0$. Absurdo!

Assim sendo $\left(\Lambda_{0}, \Lambda_{1}, \Lambda_{2}, \Lambda_{3}\right) \neq(0,0,0,0)$ conforme havia sido afirmado. Dessa forma, podemos escrever $T C$ como a união de $T C_{1}, T C_{2}$ e $T C_{3}$.

\subsubsection{Estudo dos $T C^{\prime}$ 's}

Nesta seção vamos mostrar que para todo $(\delta, \lambda) \in \Delta$ fixo, o conjunto $T C$ é um plano, $T C_{3}$ consiste de um ponto, $T C_{2}$ é uma reta menos um ponto e $T C_{1}$ é um plano menos uma reta. Todos esses conjuntos unidos das bifurcações mais degeneradas serão conexos e não vazios em $\mathcal{W}$

Comecemos notando que os pontos de $T C$ são justamente aqueles nos quais $\Lambda_{0}=0$, igualdade que pode ser reescrita como:

$$
\alpha+\lambda \beta=\frac{\lambda}{\delta}-\lambda^{2}
$$

que é a equação de um plano em $\mathcal{W}$ para todo $(\delta, \lambda) \in \Delta$ fixo. Além disso, como $\mathcal{W}$ é convexo, chegamos a conclusão de que $T C$ é conexo. Resta ainda mostrar que $T C$ é diferente de vazio no contexto de dados biológicos, mas isso deixaremos para mais tarde.

Agora, vamos analisar o que ocorre quando $\Lambda_{1}$ também se anula. Primeiro, notemos que $\Lambda_{1}$ pode ser reescrita como:

$$
-\frac{1}{\lambda^{2}}\left(\mu(\alpha+\lambda(\beta+\lambda))^{2}-\delta \lambda^{3} \beta-2 \alpha \lambda^{2} \delta+\lambda^{3}\right)
$$

Assumindo $\Lambda_{0}$ igual zero, temos que $\alpha+\lambda(\beta+\lambda)=\frac{\lambda}{\delta}$ e portanto, $\Lambda_{1}$ sobre TC pode ser escrita como:

$$
-\frac{1}{\lambda^{2}}\left(\mu\left(\frac{\lambda}{\delta}\right)^{2}-\delta \lambda^{3} \beta-2 \alpha \lambda^{2} \delta+\lambda^{3}\right)
$$

Note que esta última equação corresponde a um plano em $\mathcal{W}$. Quando este plano intersecta TC, tem-se o conjunto no qual $\Lambda_{0}$ e $\Lambda_{1}$ se anulam simultaneamente. Agora, como o termo 
que multiplica $\mu$ é diferente de zero, temos que este novo plano e TC são sempre transversais e portanto a sua intersecção é uma reta. Tal reta é conexa por causa da convexidade de $\mathcal{W}$. Mostraremos a posteriori que tal reta é não vazia em parâmetros válidos. Assim, $T C_{1}$ é um plano menos uma reta.

Agora vamos tratar do caso em que $\Lambda_{0} \Lambda_{1}$ e $\Lambda_{2}$ se anulam simultaneamente. Afirmamos que, para $(\delta, \lambda) \in \Delta$ fixo, esse conjunto é de apenas um ponto em $\mathcal{W}$. De fato, vamos achar esse ponto. Em primeiro lugar, para que $\Lambda_{0}=0$ devemos ter:

$$
\alpha=\frac{\lambda}{\delta}-\lambda(\beta+\lambda)=\alpha_{2}
$$

Substituindo esse valor em $\Lambda_{1}$ e $\Lambda_{2}$ obtemos:

$$
\begin{gathered}
\Lambda_{1}=-\left(\frac{1}{\delta^{2}} \mu+\frac{\left.\left(-\delta^{2} \lambda+\delta^{3} \lambda \beta+2\right) \delta^{3} \lambda^{2}\right)}{\delta^{2}}\right) \\
\Lambda_{2}=-\frac{6 \lambda(4-2 \delta \beta-4 \delta \lambda)}{\delta^{2}} \mu-\frac{6 \lambda\left(-\delta^{2} \lambda+\delta^{3} \lambda \beta+\delta^{3} \lambda^{2}\right)}{\delta^{2}}
\end{gathered}
$$

Da primeira equação, chegamos a $\mu=-\lambda \delta^{2}(-1+\delta \beta+2 \delta \lambda)$. Já da segunda chegamos a $4-2 \delta \beta-4 \delta \lambda=0$ ou $\mu=-\frac{1}{2} \frac{\lambda \delta^{2}(-1+\delta \beta+\delta \lambda)}{2-\delta \beta-\delta \lambda}$. Caso $4-2 \delta \beta-4 \delta \lambda=0$, então $\beta=\frac{2-2 \delta \lambda}{\delta} \mathrm{e}$, substituindo esse valor na primeira equação, chegamos que:

$$
\Lambda_{1}=\frac{-1}{\delta^{2}}\left(\mu+\delta^{2} \lambda\right)
$$

que é sempre estritamente menor que zero. Logo podemos assumir $4-2 \delta \beta-4 \delta \lambda \neq 0$ e portando devemos ter:

$$
\begin{gathered}
\mu=-\lambda \delta^{2}(-1+\delta \beta+2 \delta \lambda) \\
\mu=-\frac{1}{2} \frac{\lambda \delta^{2}(-1+\delta \beta+\delta \lambda)}{2-\delta \beta-\delta \lambda}
\end{gathered}
$$

agora, para que esse sistema seja satisfeito, $\beta$ deve assumir um dos seguintes valores:

$$
\begin{aligned}
& \beta_{1}=\frac{-2 \delta \lambda+\frac{5}{4}+\frac{1}{4} \sqrt{8 \delta \lambda+1}}{\delta} \\
& \beta_{2}=\frac{-2 \delta \lambda+\frac{5}{4}-\frac{1}{4} \sqrt{8 \delta \lambda+1}}{\delta}
\end{aligned}
$$

assim, chamaremos de $\mu_{1}$ o valor assumido por $\mu$ quando substituimos $\beta$ por $\beta_{1}$ e de $\mu_{2}$ quando substituirmos $\beta$ por $\beta_{2}$. Seus valores são:

$$
\begin{aligned}
& \mu_{1}=-\frac{1}{4} \delta^{2} \lambda(1+\sqrt{8 \delta \lambda+1}) \\
& \mu_{2}=\frac{1}{4} \delta^{2} \lambda(-1+\sqrt{8 \delta \lambda+1})
\end{aligned}
$$


note que $\mu_{1}<0$ e portanto não é um parâmetro válido em nosso estudo. Assim, devemos ter $\beta=\beta_{2}$ e $\mu=\mu_{2}$. Substituindo $\mu$ por $\mu_{2}$ e $\beta$ por $\beta_{2}$ em $\alpha_{2}$ obtemos:

$$
\alpha_{2}=\frac{1}{4} \frac{\lambda(4 \delta \lambda-1+\sqrt{8 \delta \lambda+1})}{\delta}
$$

claramente, $\alpha_{2}>0$ e $\mu_{2}>0$. Agora, para que esses parâmetros sejam válidos, devemos ter $\beta_{2}>-2 \sqrt{\alpha_{2}}$. De fato:

$$
\beta_{2}+2 \sqrt{\alpha_{2}}=-\frac{1}{4} \frac{8 \delta \lambda-5+\sqrt{8 \delta \lambda+1}-4 \sqrt{\lambda \delta(4 \lambda \delta-1+\sqrt{8 \delta \lambda+1})}}{\delta}
$$

que é sempre maior do que zero para $\delta>0$ e $\lambda>0$.

Assim, temos que $p_{2}=\left(\alpha_{2}, \beta_{2}, \mu_{2}\right) \in \mathcal{W}$ é o único ponto contido em $T C_{3}$ o que implica que $T C_{3}$ é não vazio e conexo. Além disso, em nossa demonstração mostramos que $\alpha_{2}>0$, $\mu_{2}>0$ e $\beta_{2}>-2 \sqrt{\alpha_{2}}$ e portanto $p_{2}$ é um ponto no interior de $\mathcal{W}$. Assim, pela continuidade $\operatorname{dos} \Lambda$ 's $T C_{2}$ e $T C_{1}$ são não vazios.

\subsubsection{Estudo das condições de transversalidade}

Apesar de estarmos chamando nossas bifurcações de transcríticas, esse é um abuso enquanto não demonstrarmos as condições de transversalidade. Para isso, devemos mostrar que:

- Para $T C_{1}$ ser transcrítica, o Jacobiano de $\Lambda_{0}$ em relação a $(\alpha, \beta, \mu)$ deve ter posto máximo quando calculado sobre $T C_{1}$

- Para $T C_{2}$ ser transcrítica de codimensão dois, o Jacobiano de $\left(\Lambda_{0}, \Lambda_{1}\right)$ em relação a $(\alpha, \beta, \mu)$ deve ter posto máximo quando calculado sobre $T C_{2}$

- Para $T C_{3}$ ser transcrítica de codimensão 3, o Jacobiano de $\left(\Lambda_{0}, \Lambda_{1}, \Lambda_{2}\right)$ em relação a $(\alpha, \beta, \mu)$ deve ter posto máximo quando calculado sobre $T C_{3}$

A intenção dessa secção é justamente provar isso. Em todos as partes da prova consideramos $(\delta, \lambda) \in \Delta$ fixo. 
Vamos começar mostrando que o jacobiano de $\Lambda_{0}$ aplicado na normal de TC nunca se anula. De fato, o Jacobiano de $\Lambda_{0}$ aplicado a normal $(1, \lambda, 0)$ nos fornece:

$$
\frac{\delta\left(1+\lambda^{2}\right)}{\lambda}
$$

que é, claramente, diferente de zero para todos os parâmetros admissíveis. Assim sendo, $T C_{1}$ é de fato um conjunto de pontos de bifurcação transcrítica de codimensão um.

Agora considere $\Phi$ a aplicação que leva $(\alpha, \beta, \mu)$ em $\left(\Lambda_{0}, \Lambda_{1}\right)$. Vamos mostrar que o seu jacobiano em relação a $(\alpha, \beta, \mu)$ tem posto máximo. De fato, considerando que $\Lambda_{0}=0$ e $\Lambda_{1}=0$ temos que $\alpha=-\lambda \beta+\frac{\lambda}{\delta}-\lambda^{2}=\alpha_{0}$ e $\mu=\lambda \delta^{2}(1-\delta(2 \lambda+\beta))=\mu_{0}$. Fazendo essas substituições no jacobiano, obtemos:

$$
\left[\begin{array}{ccc}
\frac{\delta}{\lambda} & \delta & 0 \\
2 \delta^{2}(\beta+2 \lambda) & \lambda \delta(4 \lambda \delta+2 \delta \beta-1) & -\delta^{-2}
\end{array}\right]
$$

que claramente tem posto dois. Assim sendo, $T C_{2}$ é de fato um conjunto de pontos de bifurcação transcrítica de codimensão dois.

Seja $\Psi$ a aplicação que leva $(\alpha, \beta, \mu)$ em $\left(\Lambda_{0}, \Lambda_{1}, \Lambda_{2}\right)$. Precisamos mostrar que o jacobiano desta aplicação sobre $T C_{3}$ é de posto máximo. Considerando que $\Lambda_{0}=0, \Lambda_{1}=0$ e $\Lambda_{2}=0$ estamos sobre $p_{2}=\left(\alpha_{2}, \beta_{2}, \mu_{2}\right)=\left(\frac{1}{4} \frac{\lambda(4 \delta \lambda-1+\sqrt{8 \delta \lambda+1})}{\delta}, \frac{-2 \delta \lambda+\frac{5}{4}-\frac{1}{4} \sqrt{8 \delta \lambda+1}}{\delta}, \frac{1}{4} \delta^{2} \lambda(-1+\sqrt{8 \delta \lambda+1})\right)$ que foi deduzido na secção anterior. Tomando o jacobiano de $\Psi$ e tomando ele em $p_{2}$ :

$$
\left[\begin{array}{ccc}
\frac{\delta}{\lambda} & \delta & 0 \\
-1 / 2 \delta(-5+\sqrt{1+8 \lambda \delta}) & -1 / 2 \delta \lambda(-3+\sqrt{1+8 \lambda \delta}) & -\delta^{-2} \\
-3 / 2 \delta \lambda(-9+5 \sqrt{1+8 \lambda \delta}+4 \lambda \delta) & -\frac{3 \lambda^{2} \delta(-1+\sqrt{1+8 \lambda \delta})(7+\sqrt{1+8 \lambda \delta})}{4} & -3 \frac{\lambda(3+\sqrt{1+8 \lambda \delta})}{\delta^{2}}
\end{array}\right]
$$

cujo determinante vale:

$$
6 \lambda \sqrt{1+8 \lambda \delta}
$$

que é diferente de zero para todos os parâmetros admissíveis. Assim sendo, $T C_{3}$ é de fato um conjunto de pontos de bifurcação transcrítica de codimensão três. 
Teorema 3.1.6. (Plano Transcrítico) Para todo $(\delta, \lambda) \in \Delta$ o conjunto de bifurcação do sistema em $\mathcal{W}$ associado ao ponto $C=\left(\frac{1}{\lambda}, 0\right)$ possui um ponto transcrítico de codimensão 3 $\left(T C_{3}\right)$, duas semi-retas conexas transcríticas de codimensão $2\left(T C_{2}\right)$ subordinadas a $T C_{3} e$ dois semi-planos conexos transcríticos de codimensão 1 ( $\left.T C_{1}\right)$ subordinadas a $T C_{2}$ e $T C_{3}$. $A$ união de $T C_{1}, T C_{2}$ e $T C_{3}$ é um plano em $\mathcal{W}$ que chamaremos de $T C$. Os conjuntos $T C_{1}$, $T C_{2}$ e $T C_{3}$ são não vazios para todo o $(\delta, \lambda) \in \Delta$. Não ocorrem mais degenerescências.

\subsection{Estudo da variedade central associada a $C$}

Vamos analisar a configuração do espaço de fase em torno de $C$. Para isso, não nos basta achar a superfície bifurcação, precisamos calcular o sinal do termo linear, quadrático, cúbico e quártico relativos à variedade central associada a $C$.

Começamos da mesma forma que anteriormente. Localizamos o ponto $C$ através da mudança de coordenadas:

$$
\begin{gathered}
x=u+\frac{1}{\lambda} \\
y=v
\end{gathered}
$$

lembremos da linearização do sistema em $C$ :

$$
A=\left[\begin{array}{rr}
-1 & \frac{\lambda}{\alpha+\lambda(\beta+\lambda)} \\
0 & -\delta+\frac{\lambda}{\alpha+\lambda(\beta+\lambda)}
\end{array}\right],
$$

Como já havíamos dito, o vetor $(1,0)$ é sempre autovetor de $A$ associado ao autovalor -1, que nunca se anula. As separatrizes relativas a esse autovetor se encontram no eixo $y=0$. Já o outro autovalor vale:

$$
-\delta+\frac{\lambda}{\alpha+\lambda(\beta+\lambda)}
$$

quando igualamos este autovalor a zero temos a equação implícita de $T C$. É fácil ver que abaixo desse plano, $C$ é uma sela, enquanto acima dele, $C$ é um nó atrator. Assim, devemos prosseguir para a análise do que ocorre quando estamos sobre a TC. Para isso, fazendo uso do teorema da variedade central, precisamos analisar uma variedade central $W^{c}(C)$, parametrizada por $w$. Temos:

$$
\dot{w}=w\left(\Gamma_{0}+\Gamma_{1} w+\Gamma_{2} w^{2}+\Gamma_{3} w^{3}+\mathcal{O}\left(w^{4}\right)\right)
$$


Já sabemos que $\Gamma_{0}=-\delta+\frac{\lambda}{\alpha+\lambda(\beta+\lambda)}$. Agora, precisamos descobrir quem são $\Gamma_{1}, \Gamma_{2}$ e $\Gamma_{3}$. Para isso, devemos fazer explicitamente a mudança de variáveis de forma a localizar a variedade central no eixo $w$. Como queremos calcular a expansão até a quarta ordem, só precisamos tornar o eixo $w$ tangente até a quarta ordem à variedade central. Assim, vamos prosseguir da seguinte forma:

- Vamos achar uma parametrização para a variedade central até quarta ordem;

- Vamos fazer uma mudança de variáveis que torne o eixo $w$ tangente a variedade central até quarta ordem;

- Vamos calcular $\Gamma_{1}, \Gamma_{2}$ e $\Gamma_{3}$ explicitamente;

- Vamos tornar as as suas expressões mais simples com auxílio dos coeficientes calculados na secção anterior.

\subsubsection{Calculo de $\Gamma_{1}, \Gamma_{2}$ e $\Gamma_{3}$}

Quando $\Gamma_{0}=0$ temos uma variedade central $W^{c}(C)$ associada ao autovalor $-\delta+\frac{\lambda}{\alpha+\lambda(\beta+\lambda)}$ e, consequentemente, ao autovetor $\left(\frac{\lambda}{(\delta-1)(\alpha+\lambda(\beta+\lambda))-\lambda}, 1\right)$. Como este autovetor é sempre LI com o vetor $(1,0)$, pelo teorema da variedade central aplicado em $C$, podemos parametrizar a variedade central como uma curva $\gamma(v)=(p(v), v)$, onde $p(v)=a_{1} v+a_{2} v^{2}+a_{3} v^{3}+a_{4} v^{4}+$ $\mathcal{O}\left(v^{5}\right)$. Vamos calcular $a_{1}, a_{2}, a_{3}$ e $a_{4}$. Para isso, tome:

$$
N(q(v))=(\dot{u}, \dot{v}) \times D(q(v), v)
$$

$N$ corresponde ao determinante da matriz formada pela derivada da curva $(q(v), v)$ e pelo campo $(\dot{u}, \dot{v})$. Assim: $q(v)=p(v)$ se, e somente se, a curva $(q(v), v)$ for uma órbita do sistema e portanto, uma variedade central. Logo, podemos calcular $a_{1}, a_{2}, a_{3}$ e $a_{4}$ usando a equação $N=0$.

Munido dos primeiros termos de $p(v)$, faremos a seguinte mudança de coordenadas:

$$
\begin{gathered}
z=u-\left(a_{1} v+a_{2} v^{2}+a_{3} v^{3}+a_{4} v^{4}\right) \\
w=v
\end{gathered}
$$


Como nos conseguimos calcular explicitamente $p(v)$ até a quarta ordem, o eixo $w$ é tangente até a quarta ordem à variedade central. Isso será suficiente para nosso cálculo. Tomando a componente do campo relativa ao eixo $w$, igualando $z$ a zero e expandindo essa expressão em termo de $w$, obtemos os termos $\Gamma_{1}, \Gamma_{2}$ e $\Gamma_{3}$.

Tais termos ainda são pouco elegantes. Para extrair informações deles, precisamos encontrar expressões mais simples. Na secção anterior, calculamos os termos $\Lambda_{0}, \Lambda_{1}, \Lambda_{2}$ e $\Lambda_{3}$ que são mais elegantes e concisos. Quando estivermos sobre $T C$, sabemos que $\Lambda_{1}$ se anula, se, e somente se, $\Gamma_{1}$ se anula. Da mesma forma, para $T C_{2}$ e $T C_{3}$. Assim, quando estivermos sobre $T C, \Gamma_{1}$ deve ser um múltiplo de $\Lambda_{1}$, quando estivermos sobre $T C_{2}, \Gamma_{2}$ um múltiplo de $\Lambda_{2}$ e quando estivermos sobre $T C_{3}, \Gamma_{3}$ um múltiplo de $\Lambda_{3}$. Como só nos é interessante $\Gamma_{i}$ caso $\Gamma_{j}=0$ com $j<i$, podemos considerar, para efeito do cálculo da configuração topológica de $C$, que $\Gamma_{i}$ é um múltiplo de $\Lambda_{i}$. Alias, a única informação que precisamos para o estudo da dinâmica é o sinal de $\Gamma_{i}$ em cada região conexa de $T C_{i}$. Assim, vamos chamar de $\left(\mathbb{Z}_{i}\right)_{i \leq 4}$ os coeficientes que possuem o mesmo sinal de $\Gamma_{i}$ em $T C_{i}$. Esses termos são:

$$
\begin{array}{ccc}
\mathbb{Z}_{1}=\Lambda_{1}= & -\left(\mu \alpha^{2}+\lambda^{4} \mu+2 \beta \lambda^{3} \mu+\beta^{2} \lambda^{2} \mu+\lambda^{3}-\beta l^{3} \delta-2 \alpha \lambda^{2} \delta+2 \alpha \mu \beta \lambda+2 \alpha \mu \lambda^{2}\right)\left(\lambda^{-2}\right) \\
\mathbb{Z}_{2}=-\Lambda_{2}= & \left(36 \alpha \mu \beta \lambda+24 \mu \alpha^{2}+12 \beta^{2} \lambda^{2} \mu-6 \alpha \lambda^{2} \delta+12 \beta \lambda^{3} \mu+24 \alpha \mu \lambda^{2}\right)\left(\lambda^{-1}\right) \\
\mathbb{Z}_{3}=\Lambda_{3}= & -24 \mu\left(6 \alpha \lambda \beta+\beta^{2} \lambda^{2}+6 \alpha^{2}+2 \alpha \lambda^{2}\right)
\end{array}
$$

De fato:

- Quando estamos sobre $T C$, temos $\Lambda_{0}=0$, o que implica $\alpha=-\lambda \beta+\frac{\lambda}{\delta}-\lambda^{2}=\alpha_{0}$ e substituindo esse valor em $\frac{\Gamma_{1}}{\Lambda_{1}}$, temos que $\frac{\Gamma_{1}}{\Lambda_{1}}=\delta^{2}$ que é constante positivo. Assim, podemos tomar $\mathbb{Z}_{1}=\Lambda_{1}$ que será suficiente para nossos propósitos.

- Substituindo $\alpha_{0}$ em $\Lambda_{1}=0$, temos que $\mu=\lambda \delta^{2}(1-\delta(2 \lambda+\beta))=\mu_{0}$. Assim, substituindo $\alpha$ por $\alpha_{0}$ e $\mu$ por $\mu_{0}$ em $\frac{\Gamma_{2}}{\Lambda_{2}}$ temos: $\frac{\Gamma_{2}}{\Lambda_{2}}=-\frac{\delta^{3}}{6}$ que é constante negativa. Assim, podemos tomar $\mathbb{Z}_{2}=-\Lambda_{2}$ que será suficiente para nossos propósitos.

- No último caso, podemos simplesmente aplicar o ponto:

$$
p_{2}=\left(\frac{1}{4} \frac{\lambda(4 \delta \lambda-1+\sqrt{8 \delta \lambda+1})}{\delta}, \frac{-2 \delta \lambda+\frac{5}{4}-\frac{1}{4} \sqrt{8 \delta \lambda+1}}{\delta}, \frac{1}{4} \delta^{2} \lambda(-1+\sqrt{8 \delta \lambda+1})\right)
$$


em $\frac{\Gamma_{3}}{\Lambda_{3}}$. Assim, obtemos:

$$
\frac{\Gamma_{3}}{\Lambda_{3}}=\frac{(3 / 2 \delta \lambda+1 / 4-1 / 4 \sqrt{8 \delta \lambda+1}+5 / 6 \sqrt{8 \delta \lambda+1} \delta \lambda) \delta^{4}}{(-1+\sqrt{8 \delta \lambda+1})(20 \delta \lambda+1+7 \sqrt{8 \delta \lambda+1})}
$$

Esta expressão é constante para cada $(\delta, \lambda) \in \Delta$ fixos e é positiva. Portanto podemos tomar $\mathbb{Z}_{3}=\Lambda_{3}$.

Agora, podemos seguir para o estudo da configuração topológica de $C$.

\subsection{Diagrama de bifurcação próximo a $C$}

Nesta secção queremos localizar e explicitar todas as configurações topológicas do ponto $C$. Além disso, uma bifurcação do tipo $T C_{3}$ possui uma curva de sela-nó de codimensão dois subordinada a ela. Queremos estudar a posição geométrica dessa curva.

\subsubsection{Os estratos}

Nossa análise será feita fixando $(\delta, \lambda) \in \Delta$ e será próxima a $T C_{3}$. Vamos começar definindo a notação das regiões onde não existe mudança de configuração topológica de $C$ (estratos). Em seguida analisaremos cada uma dessas regiões.

Para começar, notemos que o plano $T C$ evolui como uma reta no plano $(\alpha, \beta)$ e livremente em $\mu$. Agora, fixado $\left(\alpha_{0}, \beta_{0}, \mu_{0}\right)$, existe $\alpha_{T C}$ tal que $\left(\alpha_{T C}, \beta_{0}, \mu_{0}\right) \in T C$. Diremos que $\left(\alpha_{0}, \beta_{0}, \mu_{0}\right)$ está abaixo de $T C$ caso $\alpha_{0}<\alpha_{T C}$. Dessa forma, definimos:

- $C_{a}$ é o conjunto de parâmetros abaixo do plano $T C$;

- $C_{b}$ é o conjunto de parâmetros acima do plano $T C$;

Agora, quando estivermos sobre $T C$, temos a reta $T C_{2}$ dividindo o plano em dois estratos diferentes. Note que a reta $T C_{2}$ possui uma inclinação não infinita com respeito a $\mu$. Assim, fixado $\left(\alpha_{0}, \beta_{0}, \mu_{0}\right) \in T C$, existe $\mu_{T C_{2}}$ tal que $\left(\alpha_{0}, \beta_{0}, \mu_{T C_{2}}\right) \in T C_{2}$. Diremos que $\left(\alpha_{0}, \beta_{0}, \mu_{0}\right)$ está abaixo de $T C_{2}$ caso $\mu_{0}<\mu_{T C_{2}}$. Dessa forma, definimos: 


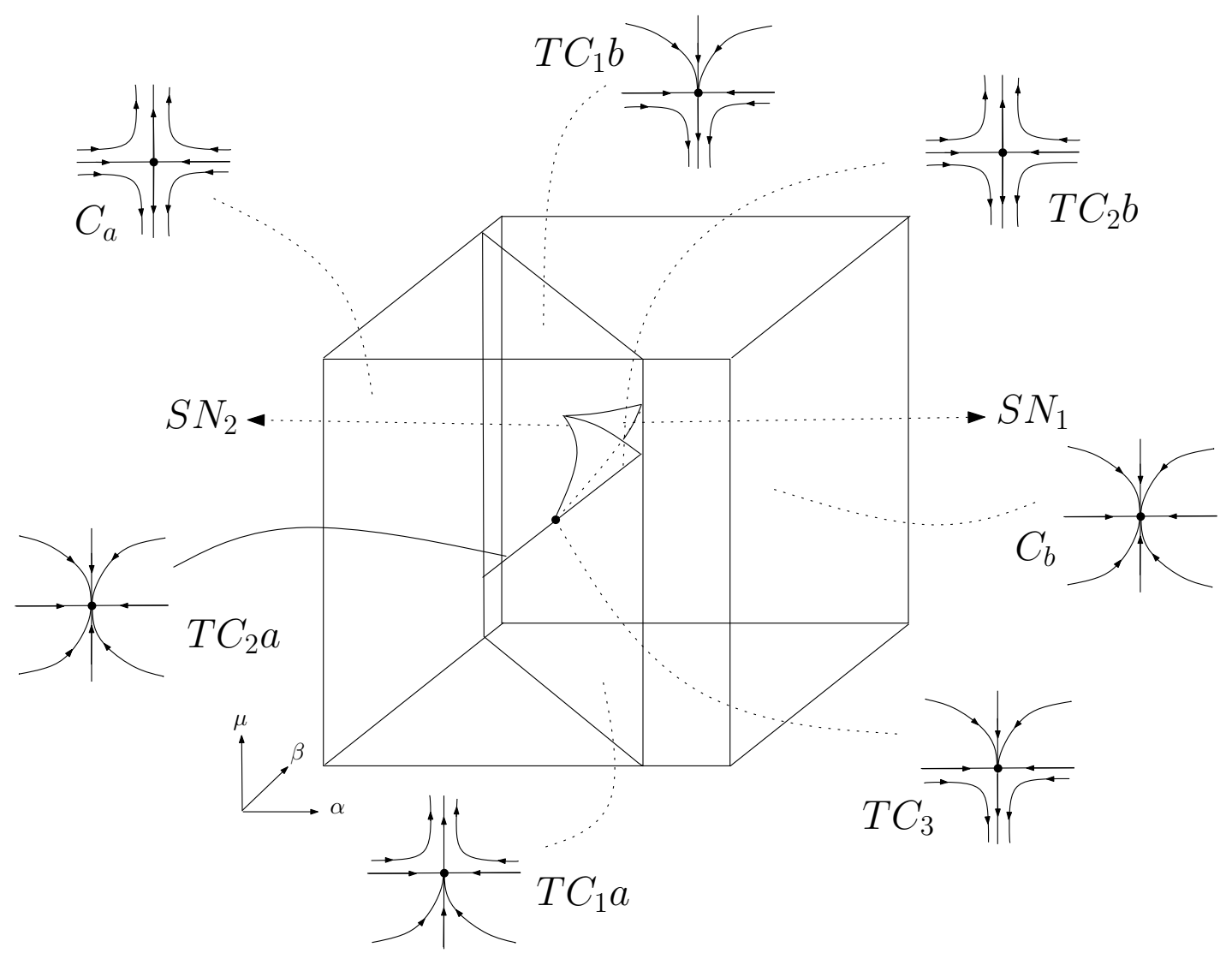

Figura 3.1: Configuração topológica de $C$ em cada região. O desenho prioriza $T C$ e $S N_{2}$, sendo que uma das componentes de $S N_{1}$ não foi desenhada, de forma a não carregar o desenho. Além disso, o desenho é ilustrativo e não foi feito com um $(\delta, \lambda)$ específico. No entanto, a menos de mudanças nas inclinações de $T C$ e $T C_{2}$, mudanças de escala e mudanças de caixa (para englobar $\beta<0)$, a bifurcação será como na figura. 
- $T C_{1} a$ é o conjunto de parâmetros de $T C$ abaixo da reta $T C_{2}$;

- $T C_{1} b$ é o conjunto de parâmetros de $T C$ acima da reta $T C_{2}$;

Por fim, quando estivermos em $T C_{2}$, temos o ponto $T C_{3}=\left(\alpha_{2}, \beta_{2}, \mu_{2}\right)$ dividindo essa reta em dois estratos distintos. Esse ponto já foi definido como $p_{2}=\left(\alpha_{2}, \beta_{2}, \mu_{2}\right)$. Assim, fixado $\left(\alpha_{0}, \beta_{0}, \mu_{0}\right) \in T C_{2}$, diremos que este ponto está abaixo de $T C_{3}$ caso $\alpha_{0}<\alpha_{2}$. Dessa forma, definimos:

- $T C_{2} a$ é o conjunto de parâmetros de $T C_{2}$ abaixo do ponto $T C_{3}$;

- $T C_{2} b$ é o conjunto de parâmetros de $T C_{2}$ acima do ponto $T C_{3}$;

Para visualizar bem estas regiões, ver figura 3.1.

\subsubsection{A configuração topológica local de $C$}

Agora, queremos estudar a configuração topológica local de $C$ em cada uma dessas regiões. Como cada uma delas é conexa e não ocorrem mais bifurcações (sobre o ponto $C$ !), a configuração topológica de $C$ não muda ao longo das regiões acima definidas. Observado isso, temos que:

Proposição 3.3.3. As configurações topológicas locais de $C$ são:

- em $C_{a}, C$ é uma sela.

- em $C_{b}, C$ é um nó atrator.

- em $T C_{1} a, C$ é uma sela-nó cuja parte nodal esta voltada para $y<0$.

- em $T C_{1} b, C$ é uma sela-nó cuja parte nodal esta voltada para $y>0$.

- em $T C_{2} a, C$ é um nó atrator não hiperbólico.

- em $T C_{2} b, C$ é uma sela não hiperbólica.

- em $T C_{3}, C$ é uma sela-nó cuja parte nodal esta voltada para y $>0$. 
Demonstração. Como a definição das componentes não depende de $(\delta, \lambda)$ e as superfícies de bifurcação não se alteram ao mudarmos $(\delta, \lambda)$, podemos fixar $(\delta, \lambda) \in \Delta$ e estudar os estratos de $\mathcal{W}$ relativos a esse ponto. Assim, para facilitar os calculos, vamos assumir que $\delta \lambda<\frac{3}{8}$. Dessa forma, teremos uma figura parecida com a 3.1, uma vez que $T C_{3}$ ocorre para $\beta>0$.

Lembremos que para $(\alpha, \beta, \mu) \in T C$ devemos ter:

$$
\alpha=-\frac{\lambda(\delta \beta+\delta \lambda-1)}{\delta}
$$

Pelas definições de $C_{i}$, caso $\alpha<-\frac{\lambda(\delta \beta+\delta \lambda-1)}{\delta}$ estamos em $C_{a}$. Assim:

- É fácil ver que a origem é um ponto que pertence a $C_{a}$. Como:

$$
\Gamma_{0}(0,0,0)=\frac{1-\delta \lambda}{\lambda}
$$

é maior que zero por hipótese, $C$ é uma sela. Dessa forma, em todos os pontos de $C_{a}$, $C$ é uma sela.

- Tomando $\alpha=\frac{\lambda}{\delta}$ e $\beta=\lambda$, o ponto $(\alpha, \beta, \mu)$ esté em $C_{b}$. Como:

$$
\Gamma_{0}\left(\frac{\lambda}{\delta}, \lambda, \mu\right)=-2 \frac{\lambda \delta^{2}}{1+2 \delta \lambda}<0
$$

esse ponto é um nó atrator. Dessa forma, em todos os pontos de $C_{b}, C$ é um nó atrator.

Agora vamos proceder para estudar a configuração em TC. Assim, temos que $\alpha=-\frac{\lambda(\delta \beta+\delta \lambda-1)}{\delta}=$ $\alpha_{0}(\beta, \mu)$. Substituindo em $\mathbb{Z}_{1}$ obtemos:

$$
-\frac{\mu-\lambda \delta^{2}+\delta^{3} \beta \lambda+2 \delta^{3} \lambda^{2}}{\delta^{2}}
$$

Logo, para $\mathbb{Z}_{1}=0$, devemos ter:

$$
\mu=-\lambda \delta^{2}(-1+\delta \beta+2 \delta \lambda)=\mu_{0}
$$

Assim, pela definição de $T C_{1} a$, um ponto pertence a esse conjunto caso $\mu<-\lambda \delta^{2}(-1+\delta \beta+2 \delta \lambda)$. Assim: 
- Não é difícil ver que o ponto $\left(\alpha_{0}(0,0), 0,0\right)$ pertence a $T C_{1} a$. Como:

$$
\mathbb{Z}_{1}\left(\alpha_{0}(0,0), 0,0\right)=\lambda(1-2 \delta \lambda)
$$

é maior que zero por hipótese. Dessa forma, como o termo quadrático é positivo, $C$ é um sela-nó cuja parte nodal está voltada para baixo, ou seja, para $y<0$. Isso é válido para todos os pontos de $T C_{1} a$.

- O ponto $\left(\alpha_{0}, 0, \lambda \delta^{2}\right)$ pertence a $T C_{1} b$. Como:

$$
\mathbb{Z}_{1}\left(\alpha_{0}\left(0, \lambda \delta^{2}\right), 0, \lambda \delta^{2}\right)=-2 \delta \lambda^{2}
$$

é menor do que zero. Dessa forma, como o termo quadrático é negativo, $C$ é um sela-nó cuja parte nodal está voltada para cima, ou seja, para $y>0$. Isso é válido para todos os pontos de $T C_{1} b$.

Agora, vamos estudar as componentes de $T C_{2}$. Esta reta é dividida pelo ponto

$$
p_{2}=\left(\frac{1}{4} \frac{\lambda(4 \delta \lambda-1+\sqrt{8 \delta \lambda+1})}{\delta}, \frac{-2 \delta \lambda+\frac{5}{4}-\frac{1}{4} \sqrt{8 \delta \lambda+1}}{\delta}, \frac{1}{4} \delta^{2} \lambda(-1+\sqrt{8 \delta \lambda+1})\right)
$$

Assim, pela definição de $T C_{2} a$, um ponto no qual $\alpha<\alpha_{2}=\frac{1}{4} \frac{\lambda(4 \delta \lambda-1+\sqrt{8 \delta \lambda+1})}{\delta}$ pertence a $T C_{2} a$.

- O ponto:

$$
\left(\frac{\lambda(3 \delta \lambda-1+\sqrt{8 \delta \lambda+1})}{4 \delta},-\frac{7 \delta \lambda-5+\sqrt{8 \delta \lambda+1}}{4 \delta}, 1 / 4 \lambda \delta^{2}(-1-\delta \lambda+\sqrt{8 \delta \lambda+1})\right)
$$

pertence a $T C_{2} a$. Como:

$$
\begin{gathered}
\mathbb{Z}_{2}\left(\frac{\lambda(3 \delta \lambda-1+\sqrt{8 \delta \lambda+1})}{4 \delta},\right. \\
\left.-\frac{7 \delta \lambda-5+\sqrt{8 \delta \lambda+1}}{4 \delta}, 1 / 4 \lambda \delta^{2}(-1-\delta \lambda+\sqrt{8 \delta \lambda+1})\right)= \\
-3 / 4 \lambda^{3} \delta(2 \sqrt{8 \delta \lambda+1}-\delta \lambda)
\end{gathered}
$$

é menor do que zero, o termo cúbico é menor do que zero e temos um nó atrator não hiperbólico. Isso é válido para todos os parâmetros em $T C_{2} a$. 
- O ponto:

$$
\left(-\frac{\lambda(\delta \lambda-1)}{\delta}, 0, \mu_{0}\left(-\frac{\lambda(\delta \lambda-1)}{\delta}, 0\right)\right)
$$

pertence a $T C_{2} b$. Como:

$$
\mathbb{Z}_{2}\left(-\frac{\lambda(\delta \lambda-1)}{\delta}, 0, \mu_{0}\left(-\frac{\lambda(\delta \lambda-1)}{\delta}, 0\right)\right)=6 \lambda^{2}(\delta \lambda-1)(8 \delta \lambda-3)
$$

é maior do que zero, o termo cúbico é maior do que zero e temos uma sela não hiperbólica. Isso é válido para todos os parâmetros em $T C_{2} b$.

Assim, falta apenas estudar o ponto $T C_{3}$. Substituindo o ponto $p_{2}$ em $\mathbb{Z}_{3}$ obtemos:

$$
\mathbb{Z}_{3}\left(p_{2}\right)=-3 / 4 \lambda^{3}(-1+\sqrt{1+8 \delta \lambda})(1+20 \delta \lambda+7 \sqrt{1+8 \delta \lambda})
$$

que é menor do que zero. Logo $C$ é uma sela-nó com a parte nodal voltada para cima, ou seja, pra $y>0$. Isso é válido para todos os parâmetros em $T C_{3}$.

Em seguida vamos estudar a curva de sela-nó de codimensão dois subordinada a $T C_{3}$.

\subsubsection{Análise da curva $S N_{2}$ subordinada a $T C_{3}$}

Vamos achar a posição geométrica da curva $S N_{2}$ associada a $T C_{3}$. Para isso, vamos estudar a dinâmica das bifurcações transcríticas próximas a $T C_{3}$. Vamos começar pela reta $T C_{2}$. Se nos restringirmos a ela, teremos apenas uma bifurcação transcrítica de codimensão um ocorrendo no ponto $T C_{3}$. Não é difícil ver que estamos nas condições da figura 3.2.

Assim, se estivermos suficientemente próximo a $T C_{3}$, temos:

- sobre $T C_{2} a$ temos um equilíbrio na região $y<0$.

- sobre $T C_{2} b$ temos um equilíbrio na região $y>0$.

Agora, tracemos duas retas nas quais $(\alpha, \beta)$ são constantes, que se encontrem em $T C$ e que sejam suficientemente próximas a $T C_{3}$ para que, nos pontos onde essas retas cortem $T C_{2}$, possamos ter os mesmo equilíbrios que já foram encontrados. Cada uma das retas deve cortar uma componente conexa diferente de $T C_{2}$. Assim, a dinâmica é como nas figuras 3.3 e 3.4.

Assim, se estivermos suficientemente próximos a $T C_{3}$, temos o seguinte: 


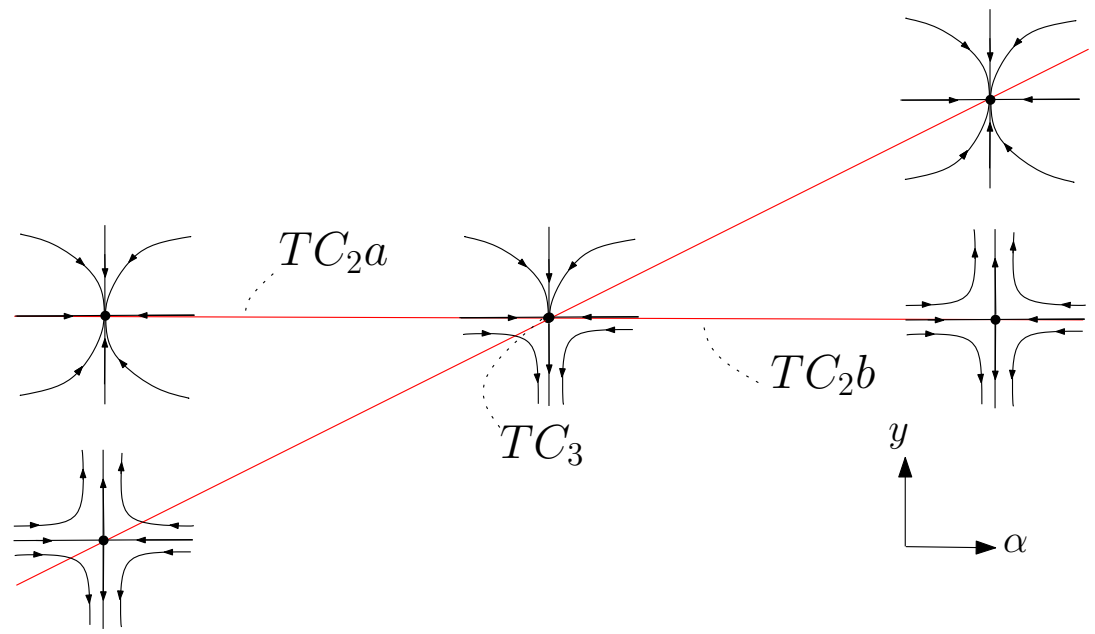

Figura 3.2: Em vermelho, temos as singularidades. A reta horizontal corresponde a $T C_{2}$. Estamos fazendo o parâmetro $\alpha$ evoluir. Apenas os equilíbrios que estão na região $y>0$ são de relevância biológica

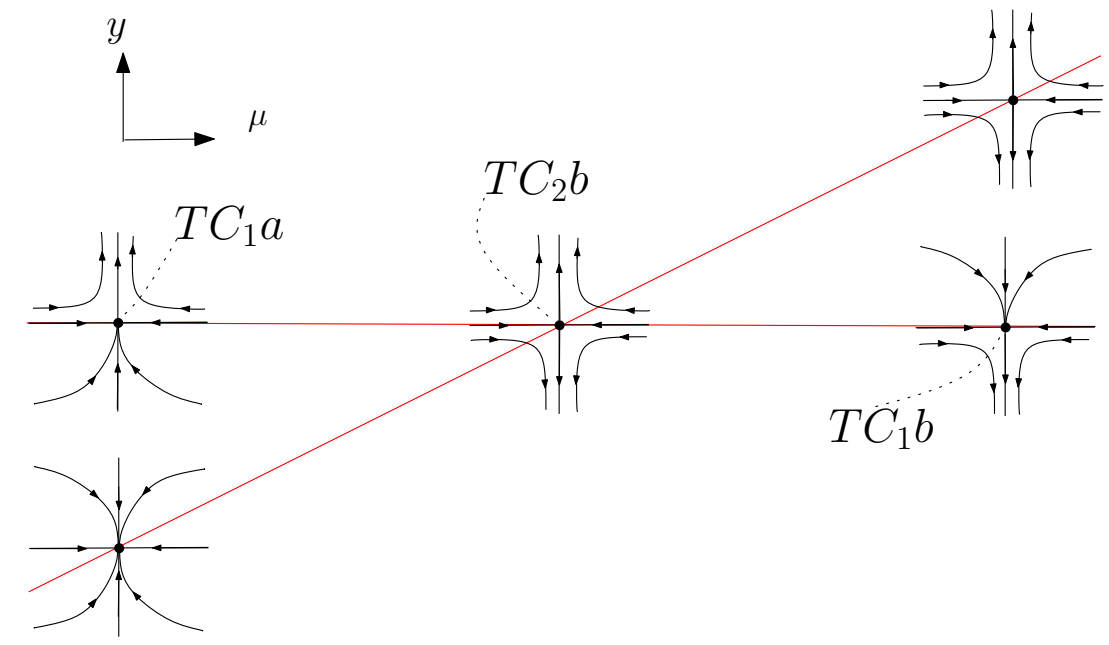

Figura 3.3: Em vermelho, temos as singularidades. A reta está em $T C$ e corta $T C_{2} b$. Estamos fazendo o parâmetro $\mu$ evoluir. Apenas os equilíbrios que estão na região $y>0$ são de relevância biológica 


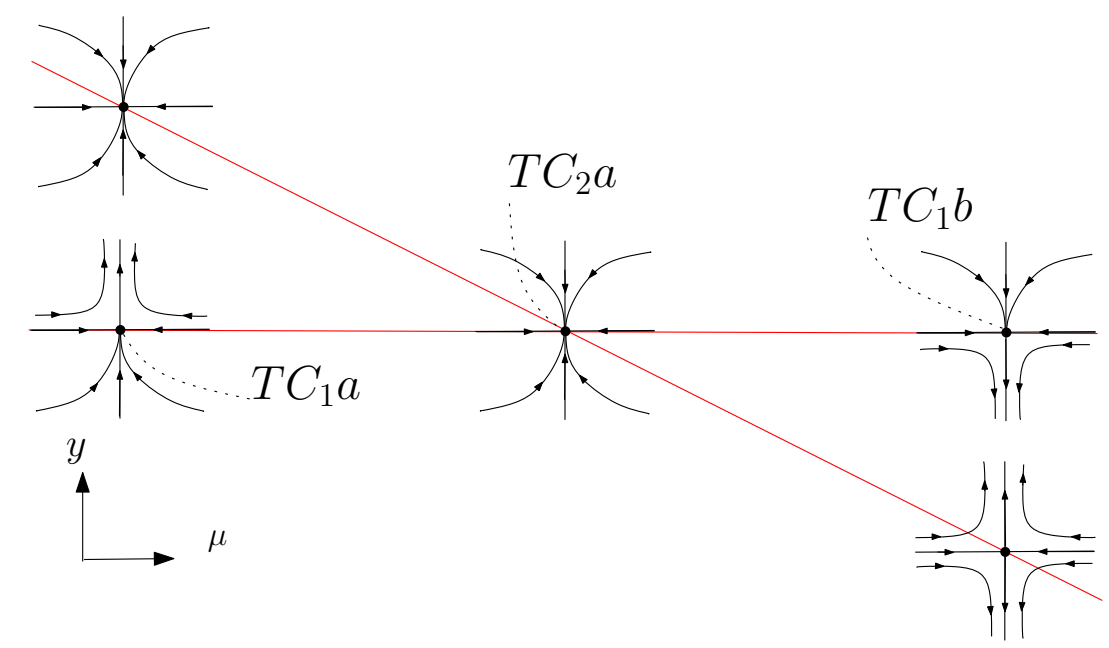

Figura 3.4: Em vermelho, temos as singularidades. A reta está em $T C$ e corta $T C_{2} a$. Estamos fazendo o parâmetro $\mu$ evoluir. Apenas os equilíbrios que estão na região $y>0$ são de relevância biológica

- em $T C_{1} a$, próximo a $T C_{2} a$, temos pelo menos um equilíbrio em $y>0$ e um em $y<0$.

- em $T C_{1}$ a, próximo a $T C_{2} b$, temos pelo menos um equilíbrio em $y>0$ e um em $y<0$.

- em $T C_{1} b$, próximo a $T C_{2} a$, temos pelo menos dois equilíbrios em $y<0$.

- em $T C_{1} b$, próximo a $T C_{2} b$, temos pelo menos dois equilíbrios em $y>0$.

Como em $T C_{1} b$ não pode haver outras bifurcações em $C$, deve ocorrer uma bifurcação do tipo sela-nó nos equilíbrios próximos a $C$. De fato isso já era esperado pelo diagrama de bifurcação de $T C_{3}$ (para maiores informações ver apêndice). Dessa forma, sabemos que existe uma curva $S N_{1}$ sobre $T C$ e que ela encontra-se em $T C_{1} b$. A parte relativa aos equilíbrios do quadrante superior encontra-se próximas a $T C_{2} b$, como ilustra-se na figura 3.5.

Dessa forma, como a curva $S N_{2}$ subordinada a $T C_{3}$ possui uma superfície $S N_{1}$ subordinada a ela que cruza $T C$ fora da reta $T C_{2}$, sabemos que $S N_{2}$ encontra-se próxima a curva $S N_{1}$ sólida da figura 3.5. Resta saber se a curva $S N_{2}$ está em $C_{a}$ ou $C_{b}$. Para isso, tomaremos uma reta transversal a $T C$ e que corte $T C$ em um ponto localizado em $T C_{b}$ suficientemente próximo a $T C_{3}$ para existirem dois equilíbrios em $y>0$. Assim, é fácil ver que a dinâmica 


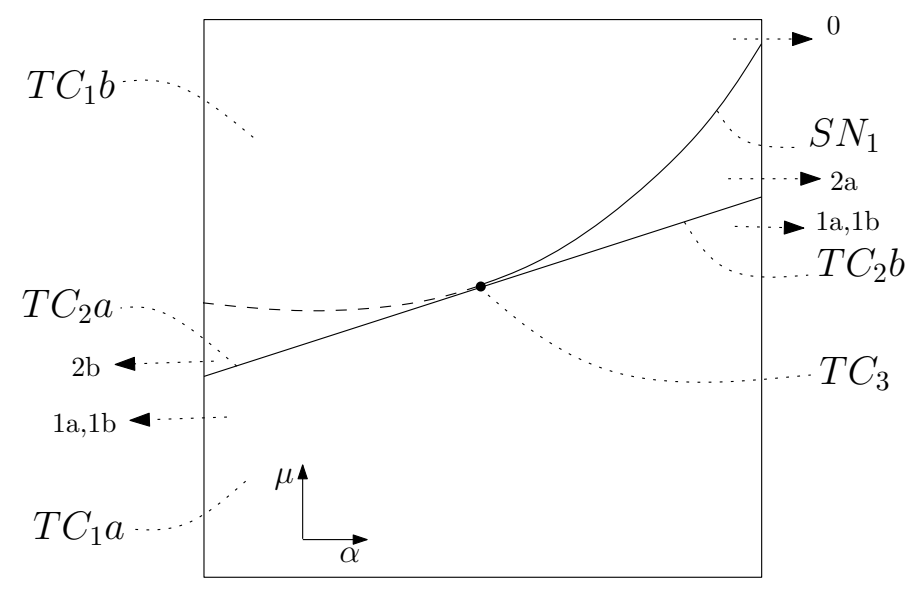

Figura 3.5: O quadrado corresponde ao plano $T C$, a reta corresponde a reta $T C_{2}$ e o ponto a $T C_{3}$. Temos a curva $S N_{1}$ separando $T C_{b}$ em três parte. Em pontilhado temos a parte de $S N_{1}$ relativa aos equilíbrios sem importância biológica. Em cada região colocamos uma legenda do número de equilíbrios nas regiões a e b: $a=\{y>0\}, b=\{y<0\}$.

deve ser a da figura 3.6.

Dessa forma, só podem existir três equilíbrios em $y>0$ próximos a $C$, caso estejamos em $C_{a}$. Assim, $S N_{2}$ encontra-se em $C_{a}$ e tem posição geométrica como na figura 3.1.

\subsection{Estudo da bifurcação transcrítica em $C \operatorname{com} \beta>0$}

Nesta secção estamos nos preparando para um estudo que será realizado a posteriori. Neste novo estudo, vamos exigir $\beta>0$. Lembremos do seguinte conjunto:

$$
\mathcal{W}_{1}=\left\{(\beta, \alpha, \mu) \in \mathbb{R}^{3} ;(\alpha, \beta, \mu) \in \mathcal{W}, \beta>0\right\}
$$

Queremos obter informações sobre a existência da superfície de bifurcação transcrítica:

Proposição 3.4.1. Para $(\delta, \lambda) \in \Delta$ fixos, o plano $T C_{1}$ cruza $\mathcal{W}_{1}$ caso $\delta \lambda<1$, a reta $T C_{2}$ cruza $\mathcal{W}_{1}$ caso $\delta \lambda<\frac{1}{2}$ e o ponto $T C_{3} \in \mathcal{W}_{1}$ caso $\delta \lambda<\frac{3}{8}$.

Demonstração. A equação implícita de TC é:

$$
0=\frac{\delta \alpha+\delta \beta \lambda+\delta \lambda^{2}-\lambda}{\lambda}
$$




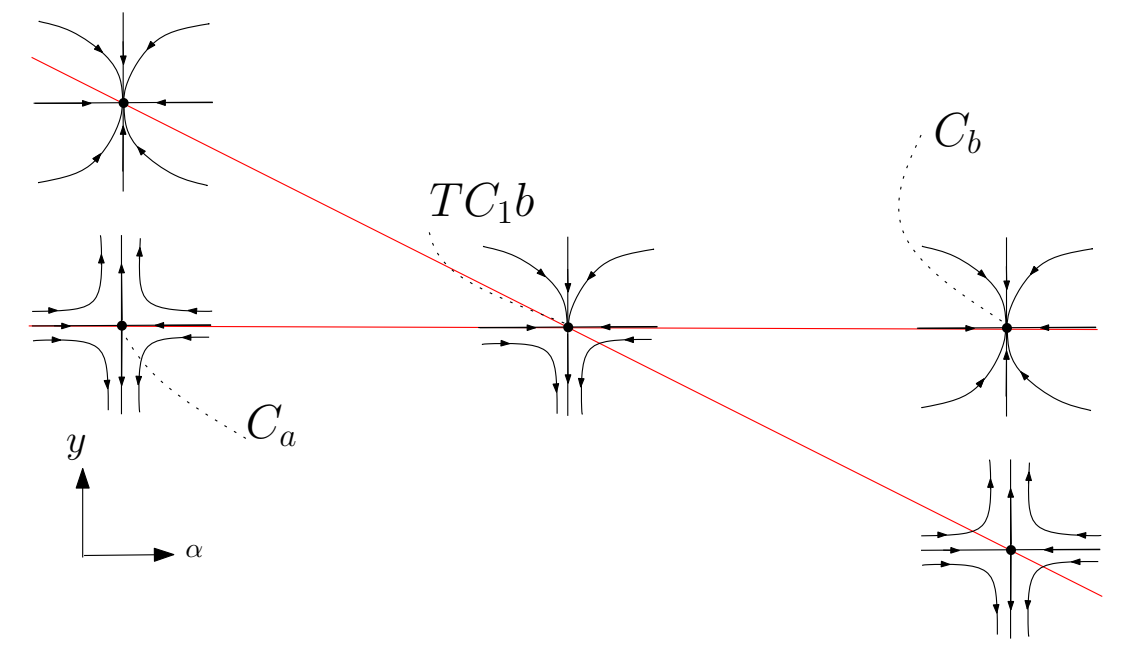

Figura 3.6: Em vermelho, temos as singularidades. A reta está é transversal a $T C$ e corta $T C_{2} b$. Estamos fazendo o parâmetro $\alpha$ evoluir. Apenas os equilíbrios que estão na região $y>0$ são de relevância biológica.

Resolvendo essa equação em função de $\beta$, obtemos:

$$
\beta=\frac{-\delta \alpha+\delta(1-\delta \lambda)}{\delta \lambda}
$$

Assim, para $\beta$ ser positiva, devemos ter:

$$
\delta \alpha<\delta(1-\delta \lambda)
$$

Como $\alpha \geq 0$, devemos ter $1-\delta \lambda>0$ para que $T C$ cruze $\mathcal{W}_{1}$.

Agora, para que ocorra $T C_{2}$, além da última equação ser satisfeita, devemos ter:

$$
-\frac{\mu \alpha^{2}+\lambda^{4} \mu+2 \beta \lambda^{3} \mu+\beta^{2} \lambda^{2} \mu+\lambda^{3}-\beta l^{3} \delta-2 \alpha \lambda^{2} \delta+2 \alpha \mu \beta \lambda+2 \alpha \mu \lambda^{2}}{\lambda^{2}}=0
$$

Substituindo nesta equação, $\alpha$ por $-\frac{\lambda(\delta \beta+\delta \lambda-1)}{\delta}$ obtemos:

$$
0=-\frac{\mu-\delta^{2} \lambda+\delta^{3} \beta \lambda+2 \delta^{3} \lambda^{2}}{\delta^{2} \lambda}
$$

resolvendo em função de $\beta$ obtemos:

$$
\beta=\frac{-\mu}{\lambda \delta^{3}}+\frac{1}{\delta}(1-2 \delta \lambda)
$$


Como $\beta$ deve ser maior que zero, temos:

$$
\frac{\mu}{\lambda \delta^{3}}<+\frac{1}{\delta}(1-2 \delta \lambda)
$$

como $\mu \geq 0$, devemos ter $1-2 \delta \lambda>0$. Assim, para que $T C_{2}$ cruze $\mathcal{W}_{1}$ caso $\delta \lambda<\frac{1}{2}$.

Agora, para ocorrer $T C_{3}$ só precisamos analisar se o ponto

$$
p_{2}=\left(\frac{1}{4} \frac{\lambda(4 \delta \lambda-1+\sqrt{8 \delta \lambda+1})}{\delta}, \frac{-2 \delta \lambda+\frac{5}{4}-\frac{1}{4} \sqrt{8 \delta \lambda+1}}{\delta}, \frac{1}{4} \delta^{2} \lambda(-1+\sqrt{8 \delta \lambda+1})\right)
$$

pertence a $\mathcal{W}_{1}$. Para tal, basta que:

$$
\frac{-2 \delta \lambda+\frac{5}{4}-\frac{1}{4} \sqrt{8 \delta \lambda+1}}{\delta}>0
$$

e, portanto, $\lambda \delta<\frac{3}{8}$. 


\section{Capítulo 4}

\section{Estudo das bifurcações do tipo sela-nó}

Neste capítulo vamos estudar as bifurcações do tipo sela-nó que ocorrem em parâmetros válidos $(\mathcal{W} \times \Delta)$. De forma a melhor organizar o estudo, o dividimos em sete secções: na primeira secção, apresentaremos a estratégia de estudo. Na segunda e terceira seç̧ões obteremos informações sobre as superfícies de selas-nó de codimensão um, e na quarta e quinta secção faremos o mesmo para as selas-nó de codimensão dois. Na sexta secção, discutiremos a condição de não-degenerescência. Por fim, na última secção, organizaremos os principais resultados em um teorema.

\subsection{Estratégia}

Para estudar a bifurcação do tipo sela-nó, comecemos notando que uma condição necessária para que essa bifurcação ocorra é $F(x)$ e $F^{\prime}(x)$ (ver capítulo 2 para a definição) serem iguais a zero. Além disso, para termos uma bifurcação do tipo sela-nó de codimensão 1, devemos satisfazer as condições de transversalidade dos parâmetros e a condição de não-degenerescência (no caso $F^{\prime \prime}(x) \neq 0$ ). Caso $F^{\prime \prime}(x)$ seja igual a zero, genericamente teremos uma bifurcação do tipo sela-nó de codimensão dois.

Neste estudo, vamos nos concentrar nas bifurcações do tipo sela-nó de codimensão um e dois. Acreditamos, com base em indícios numéricos, que não existem bifurcações de codimensão três. Além disso, dado a importância do estudo desse tipo de bifurcação, daremos 
uma demonstração para uma caixa de parâmetros baseada em análise intervalar e com ajuda computacional.

Para a analise das bifurcações de codimensão um e dois, definiremos duas aplicações: $\Psi_{1}$ (para analisar SN1) e $\Psi_{2}$ (para analisar SN2):

$$
\begin{array}{rlcc}
\Psi_{1}: \mathcal{W} \times \Delta \times \mathbb{R}_{+} & \rightarrow & \mathbb{R}^{4} \\
(\alpha, \beta, \mu, \delta, \lambda, x) & \mapsto & \left(F(x), F^{\prime}(x), \delta, \lambda\right) \\
\Psi_{2}: \mathcal{W} \times \Delta \times \mathbb{R}_{+} & \rightarrow & \mathbb{R}^{5} \\
(\alpha, \beta, \mu, \delta, \lambda, x) & \mapsto & \left(F(x), F^{\prime}(x), \delta, \lambda, F^{\prime \prime}(x)\right)
\end{array}
$$

Através dessas aplicações mostraremos a regularidade de SN1 e SN2 e que as condições de transversalidade são válidas.

\subsection{Estudo de $\Psi_{1}$}

Lembremos que a condição necessária para que um ponto seja um candidato a bifurcação selanó é que $F(x)$ e $F^{\prime}(x)$ se anulem simultaneamente. Assim sendo, o conjunto $\Psi_{1}^{-1}((0,0, \delta, \lambda))$ corresponde a todos os candidatos a bifurcação do tipo sela-nó. Vamos analisar esse conjunto.

Lema 4.2.1. A aplicação $\Psi_{1}$ restrita à $\Psi_{1}^{-1}((0,0, \delta, \lambda))$ é uma submersão.

Demonstração. Comecemos tomando o jacobiano de $\Psi_{1}$ que chamaremos de $J \Psi_{1}$. Tomamos a base canônica $e_{1}, e_{2}, \ldots, e_{6}$ do $\mathbb{R}^{6}$ e a base canônica $f_{1}, f_{2}, f_{3}, f_{4}$ do $\mathbb{R}^{4}$. Assim, temos que:

$$
\begin{gathered}
J \Psi_{1} \cdot e_{1}=\left(\begin{array}{c}
-\delta x^{2}+2 \mu(-1+\lambda x)\left(\alpha x^{2}+\beta x+1\right) x^{2} \\
-2 \delta x+\left(2 \mu \lambda x^{2}+4 \mu x(-1+\lambda x)\right)\left(\alpha x^{2}+\beta x+1\right)+2 \mu x^{2}(-1+\lambda x)(2 \alpha x+\beta) \\
0 \\
0 \\
J \Psi_{1} \cdot e_{2}=\left(\begin{array}{c}
-\delta x+2 \mu(-1+\lambda x)\left(\alpha x^{2}+\beta x+1\right) x \\
-\delta+(2 \mu \lambda x+2 \mu(-1+\lambda x))\left(\alpha x^{2}+\beta x+1\right)+2 \mu x(-1+\lambda x)(2 \alpha x+\beta) \\
0 \\
0
\end{array}\right)
\end{array}\right.
\end{gathered}
$$


4.2. ESTUDO DE $\Psi_{1}$

$$
\begin{aligned}
& J \Psi_{1} \cdot e_{3}=\left(\begin{array}{c}
(-1+\lambda x)\left(\alpha x^{2}+\beta x+1\right)^{2} \\
\lambda\left(\alpha x^{2}+\beta x+1\right)^{2}+2(-1+\lambda x)(2 \alpha x+\beta)\left(\alpha x^{2}+\beta x+1\right) \\
0 \\
0
\end{array}\right) \\
& J \Psi_{1} \cdot e_{4}=\left(\begin{array}{c}
-\left(\alpha x^{2}+\beta x+1\right) \\
-(2 \alpha x+\beta) \\
1 \\
0
\end{array}\right) \\
& J \Psi_{1} \cdot e_{5}=\left(\begin{array}{c}
\mu x\left(\alpha x^{2}+\beta x+1\right)^{2} \\
\mu\left(\alpha x^{2}+\beta x+1\right)^{2}+2 \mu x\left(\alpha x^{2}+\beta x+1\right)^{2}(2 \alpha x+\beta) \\
0 \\
1
\end{array}\right) \\
& J \Psi_{1} \cdot e_{6}=\left(\begin{array}{c}
F^{\prime}(x) \\
F^{\prime \prime}(x) \\
0 \\
0
\end{array}\right)
\end{aligned}
$$

Note que apenas em $J \Psi_{1} \cdot e_{4}$ o termo relativo a $f_{3}$ é diferente de zero. A mesma coisa acontece em $J \Psi_{1} \cdot e_{5}$ em relação à $f_{4}$. Assim, para que a aplicação seja sobrejetiva, basta que $J \Psi_{1} \cdot e_{1}, J \Psi_{1} \cdot e_{2}, J \Psi_{1} \cdot e_{3}$ e $J \Psi_{1} \cdot e_{6}$ gerem o subespaço $\left\{f_{1}, f_{2}\right\}$. Na verdade, apenas os vetores $\left\{J \Psi_{1} \cdot e_{1}, J \Psi_{1} \cdot e_{2}, J \Psi_{1} \cdot e_{3}\right\}$ já serão suficientes. De fato:

Como

$$
0=F(x)=-\delta\left(\alpha x^{2}+\beta x+1\right)+\mu(-1+\lambda x)\left(\alpha x^{2}+\beta x+1\right)^{2}+x
$$

então:

$$
2 \mu(-1+\lambda x)\left(\alpha x^{2}+\beta x+1\right)=2 \frac{x}{\left(\alpha x^{2}+\beta x+1\right)}+2 \delta
$$


e portanto:

$$
J \Psi_{1} \cdot e_{1}=\left(\begin{array}{c}
x^{2}\left(\frac{-2 x}{\alpha x^{2}+\beta x+1}+\delta\right) \\
E q 1 \\
0 \\
0
\end{array}\right)
$$

onde,

$E q 1=-2 \delta x+2 \mu \lambda x^{2}\left(\alpha x^{2}+\beta x+1\right)+4 \mu x\left(\alpha x^{2}+\beta x+1\right)(-1+\lambda x)+2 \mu x^{2}(-1+\lambda x)(2 \alpha x+\beta)$

$$
J \Psi_{1} \cdot e_{2}=\left(\begin{array}{c}
x\left(\frac{-2 x}{\alpha x^{2}+\beta x+1}+\delta\right) \\
E q 2 \\
0 \\
0
\end{array}\right)
$$

onde,

$$
E q 2=-\delta+2 \mu \lambda x\left(\alpha x^{2}+\beta x+1\right)+2 \mu\left(\alpha x^{2}+\beta x+1\right)(-1+\lambda x)+2 \mu x(-1+\lambda x)(2 \alpha x+\beta)
$$

Da mesma forma $(-1+\lambda x)\left(\alpha x^{2}+\beta x+1\right)^{2}=\frac{1}{\mu}\left(-x+\delta\left(\alpha x^{2}+\beta x+1\right)\right)$. Além disso, como $0=F^{\prime}(x)=-\delta(2 \alpha x+\beta)+\mu \lambda\left(\alpha x^{2}+\beta x+1\right)^{2}+2 \mu\left(\alpha x^{2}+\beta x+1\right)(-1+\lambda x)(2 \alpha x+\beta)+1$ então:

$$
\lambda\left(\alpha x^{2}+\beta x+1\right)^{2}+2(-1+\lambda x)(2 \alpha x+\beta)\left(\alpha x^{2}+\beta x+1\right)=\frac{1}{\mu}(\delta(2 \alpha x+\beta)-1)
$$

Assim:

$$
J \Psi_{1} \cdot e_{3}=\left(\begin{array}{c}
\frac{1}{\mu}\left(-x+\delta\left(\alpha x^{2}+\beta x+1\right)\right) \\
\frac{1}{\mu}(\delta(2 \alpha x+\beta)-1) \\
0 \\
0
\end{array}\right)
$$

Isso se $\mu \neq 0$. Trataremos o caso $\mu=0$ separadamente a posteriori. Por hora, vamos trabalhar com $\mu \neq 0$. Agora considere as seguintes funções:

$$
\begin{array}{cccc}
\psi_{1}: & \mathbb{R}^{3} & \rightarrow & \mathbb{R} \\
(A, B, C) & \mapsto & \left(A\left(J \Psi_{1} \cdot e_{1}\right)+B\left(J \Psi_{1} \cdot e_{2}\right)+C\left(J \Psi_{1} \cdot e_{3}\right)\right) \cdot f_{1}
\end{array}
$$




$$
\begin{array}{cccc}
\psi_{2}: & \mathbb{R}^{3} & \rightarrow & \mathbb{R} \\
(A, B, C) & \mapsto & \left(A\left(J \Psi_{1} \cdot e_{1}\right)+B\left(J \Psi_{1} \cdot e_{2}\right)+C\left(J \Psi_{1} \cdot e_{3}\right)\right) \cdot f_{2}
\end{array}
$$

Essas duas funções têm como núcleo planos no espaço $\mathbb{R}^{3}$. Caso os dois planos sejam distintos, então $\left\{J \Psi_{1} \cdot e_{1}, J \Psi_{1} \cdot e_{2}, J \Psi_{1} \cdot e_{3}\right\}$ gera o subespaço $\left\{f_{1}, f_{2}\right\}$. Nossa estratégia é assumir, por absurdo, que os planos são iguais e chegar em uma contradição. Comecemos por entender melhor esses dois planos:

$0=\psi_{1}(A, B, C)=A x^{2}\left(\frac{-2 x}{\alpha x^{2}+\beta x+1}+\delta\right)+B x\left(\frac{-2 x}{\alpha x^{2}+\beta x+1}+\delta\right)+C\left(\frac{1}{\mu}\left(-x+\delta\left(\alpha x^{2}+\beta x+1\right)\right)\right)$

Assim sendo, caso $\left.-x+\delta\left(\alpha x^{2}+\beta x+1\right)\right) \neq 0$ :

$$
C=\frac{A\left(x^{2}\left(\frac{-2 x}{\alpha x^{2}+\beta x+1}+\delta\right)\right)+B\left(x\left(\frac{-2 x}{\alpha x^{2}+\beta x+1}+\delta\right)\right)}{\left(\frac{1}{\mu}\left(-x+\delta\left(\alpha x^{2}+\beta x+1\right)\right)\right)}
$$

Logo, os vetores que geram o núcleo de $\psi_{1}$ são:

$$
\begin{aligned}
& v_{1}:=\left(1,0, \frac{\mu x^{2}\left(\frac{-2 x}{\alpha x^{2}+\beta x+1}+\delta\right)}{\left(-x+\delta\left(\alpha x^{2}+\beta x+1\right)\right)}\right) \\
& v_{2}:=\left(0,1, \frac{\mu x\left(\frac{-2 x}{\alpha x^{2}+\beta x+1}+\delta\right)}{\left(-x+\delta\left(\alpha x^{2}+\beta x+1\right)\right)}\right)
\end{aligned}
$$

Agora:

$$
0=\psi_{2}=A(E q 1)+B(E q 2)+C\left(\frac{1}{\mu}(\delta(2 \alpha x+\beta)-1)\right)
$$

Assim sendo, caso $\delta(2 \alpha x+\beta)-1 \neq 0$ então:

$$
C=-\mu \frac{A(E q 1)+B(E q 2)}{\delta(2 \alpha x+\beta)-1}
$$

Logo, os vetores que geram o kernel de $\psi_{2}$ são:

$$
\begin{aligned}
& w_{1}:=\left(1,0,-\mu \frac{(E q 1)}{\delta(2 \alpha x+\beta)-1)}\right) \\
& w_{2}:=\left(0,1,-\mu \frac{(E q 2)}{\delta(2 \alpha x+\beta)-1)}\right)
\end{aligned}
$$

Assim, para os dois planos serem iguais, devemos ter: $v_{1}=w_{1}$ e $v_{2}=w_{2}$. Assim, chegamos que $E q 1=x E q 2$ ou $\frac{-2 x}{\alpha x^{2}+\beta x+1}+\delta=0$. No entanto, caso $\frac{-2 x}{\alpha x^{2}+\beta x+1}+\delta=0$, então $E q 1=0=$ $E q 2$ e portanto $E q 1=x E q 2$ de qualquer forma. Assim, temos que:

$$
\mu \lambda\left(\alpha x^{2}+\beta x+1\right)^{2}+1=0
$$


o que é absurdo!

Assim, resta tratar de quatro casos que fomos assumindo como não sendo verdadeiros:

- $x=0$

- $\mu=0$

$-x+\delta\left(\alpha x^{2}+\beta x+1\right)=0$

- $\delta(2 \alpha x+\beta)-1=0$

Caso 1: $x=0$. Neste caso $F(x)=-\delta-\mu \neq 0$. Logo isso contraria a hipótese de estarmos sobre o conjunto $\Psi_{1}^{-1}(0,0, \delta, \lambda)$.

Caso 2: $\mu=0$. Substituindo $\mu$ por zero, obtemos:

$$
\begin{aligned}
& J \Psi_{1} \cdot e_{1}=\left(\begin{array}{c}
-\delta x^{2} \\
-2 \delta x \\
0 \\
0
\end{array}\right) \\
& J \Psi_{1} \cdot e_{2}=\left(\begin{array}{c}
-\delta x \\
-\delta \\
0 \\
0
\end{array}\right)
\end{aligned}
$$

Pelo caso anterior, podemos assumir $x \neq 0$. Assim sendo, temos que:

$$
\begin{aligned}
& \left\{\begin{array}{c}
\left(\frac{1}{\delta x^{2}}\right)\left(-\delta x^{2}\right)+\left(-\frac{2}{\delta x}\right)(-\delta x)=1 \\
\left(\frac{1}{\delta x^{2}}\right)(-2 \delta x)+\left(-\frac{2}{\delta x}\right)(-\delta)=0
\end{array}\right. \\
& \left\{\begin{array}{c}
\left(-\frac{1}{\delta x}\right)\left(-\delta x^{2}\right)+\left(\frac{1}{\delta}\right)(-\delta x)=0 \\
\left(-\frac{1}{\delta x}\right)(-2 \delta x)+\left(\frac{1}{\delta}\right)(-\delta)=1
\end{array}\right.
\end{aligned}
$$

Logo $f_{1}, f_{2}$ é gerado por $J \Psi_{1} \cdot e_{1}, J \Psi_{1} \cdot e_{2}$. Logo $\Psi_{1}$ é uma submersão.

Caso 3: $-x+\delta\left(\alpha x^{2}+\beta x+1\right)=0$. Isso implica que $x=\delta\left(\alpha x^{2}+\beta x+1\right)$ e portanto 
$\delta=\frac{x}{\alpha x^{2}+\beta x+1}$. Assim, $0=F(x)=\mu(-1+\lambda x)\left(\alpha x^{2}+\beta x+1\right)^{2}$. Isso implica que: ou $\mu=0$ e então estamos no caso anterior, ou $x=\frac{1}{\lambda}$. Neste caso, substituindo $x$ por $\frac{1}{\lambda}$ obtemos:

$$
\begin{gathered}
J \Psi_{1} \cdot e_{2}=\left(\begin{array}{c}
-\frac{\delta}{\lambda} \\
E q 2 \\
0 \\
0
\end{array}\right) \\
J \Psi_{1} \cdot e_{3}=\left(\begin{array}{c}
\lambda\left(\alpha\left(\frac{1}{\lambda}\right)^{2}+\beta\left(\frac{1}{\lambda}\right)+1\right) \\
0 \\
0
\end{array}\right)
\end{gathered}
$$

Note que $J \Psi_{1} \cdot e_{3} \neq(0,0,0,0)$ e portanto gera o subespaço $f_{2}$. Agora, como o termo em $f_{1}$ de $J \Psi_{1} \cdot e_{2}$ é diferente de zero, temos que ambos os vetores geram $\left\{f_{1}, f_{2}\right\}$ e portanto temos uma submersão.

Caso 4: $\delta(2 \alpha x+\beta)-1=0$. Como podemos assumir pelo caso 2 que $\mu \neq 0$, é fácil ver que:

$$
\left(J \Psi_{1} \cdot e_{3}\right) \cdot f_{2}=0
$$

Logo $(0,0,1) \in \operatorname{Núcleo}\left(\psi_{2}\right)$. Agora, para que este vetor também esteja no Núcleo de $\psi_{1}$ devemos ter: ou $\mu=0$, o que não ocorre pelo caso 2 ou $-x+\delta\left(\alpha x^{2}+\beta x+1\right)=0$ o que foi estudado no caso 3. Assim os Núcleos são diferentes e portanto a aplicação $\Psi_{1}$ é uma submersão.

\section{3 $S N_{1}$}

Já sabemos que $\Psi_{1}$ restrita ao conjunto $\Psi_{1}^{-1}(0,0, \delta, \lambda)$ é uma submersão. Fixado $\left(\delta_{0}, \lambda_{0}\right) \in \Delta$, isso significa que a superfície $\Psi_{1}^{-1}\left(0,0, \delta_{0}, \lambda_{0}\right)$ é mergulhada e fechada em $\mathcal{W} \times \mathbb{R}$.

Para ser de nosso interesse, $x$ deve ser menor ou igual a $\frac{1}{\lambda_{0}}$ e, portanto, o conjunto relevante será $\Psi_{1}^{-1}\left(0,0, \delta_{0}, \lambda_{0}\right) \cap\left(\mathcal{W} \times\left(\delta_{0}, \lambda_{0}\right) \times\left[0, \frac{1}{\lambda_{0}}\right]\right)$. Esse conjunto continua sendo uma 
superfície mergulhada fechada, mas passa a ter bordo em $x=\frac{1}{\lambda_{0}}$. Esse bordo corresponde à bifurcação transcrítica de codimensão dois. Agora, podemos definir:

$$
\begin{aligned}
\Pi: \mathcal{W} \times \Delta \times \mathbb{R}_{+} & \rightarrow \mathcal{W} \\
(\alpha, \beta, \mu, \delta, \lambda, x) & \mapsto(\alpha, \beta, \mu)
\end{aligned}
$$

Assim sendo, o conjunto que é de nosso interesse em $\mathcal{W}$ corresponde a $\Pi\left(\Psi_{1}^{-1}\left(0,0, \delta_{0}, \lambda_{0}\right) \cap\right.$ $\left.\mathcal{W} \times\left(\delta_{0}, \lambda_{0}\right) \times\left[0, \frac{1}{\lambda_{0}}\right]\right):$

Definição 4.3.1. Fixado $\left.\left(\delta_{0}, \lambda_{0}\right) \in \Delta\right)$, chamaremos de superficie de dobra geral o conjunto

$$
\Pi\left(\Psi_{1}^{-1}\left(0,0, \delta_{0}, \lambda_{0}\right) \cap \mathcal{W} \times\left(\delta_{0}, \lambda_{0}\right) \times\left[0, \frac{1}{\lambda_{0}}\right]\right)
$$

A superfície de dobra geral permanece fechada pois $\left[0, \frac{1}{\lambda_{0}}\right]$ é compacto. Vamos estudar este conjunto.

Lema 4.3.2. Um ponto da dobra geral é regular se, e somente se, ele pertence a superfície de sela-nó de codimensão um $\left(S N_{1}\right)$. Em particular, $S N_{1}$ é uma superfície imersa $C^{\infty}$ de $\mathcal{W}$.

Demonstração. Tome $\left(\alpha_{0}, \beta_{0}, \mu_{0}\right)=\Pi\left(\alpha_{0}, \beta_{0}, \mu_{0}, \delta_{0}, \lambda_{0}, x_{0}\right)$. Este ponto será regular caso a aplicação $\Pi$ restrita ao plano tangente da superfície $\Psi_{1}^{-1}\left(0,0, \delta_{0}, \lambda_{0}\right)$ em $\left(\alpha_{0}, \beta_{0}, \mu_{0}, \delta_{0}, \lambda_{0}, x_{0}\right)$ seja difeomorfismo. Isso ocorre sempre que a intersecção do subespaço gerado por $e_{4}, e_{5} \mathrm{e}$ $e_{6}$ com o plano tangente do conjunto $\Psi_{1}^{-1}\left(0,0, \delta_{0}, \lambda_{0}\right)$ for vazio. Isso é verdade sempre que $F^{\prime \prime}(x) \neq 0$.

De fato, tome um vetor nesse subespaço: $V=a e_{4}+b e_{5}+c e_{6}$. Queremos saber quando $J \Psi_{1} \cdot V \neq(0,0,0,0)$. Vamos assumir por absurdo que $J \Psi_{1} \cdot V=(0,0,0,0)$. Agora, $J \Psi_{1} \cdot e_{4}$ é o único vetor com termo $f_{3}$ não nulo e $J \Psi_{1} \cdot e_{5}$ é o único vetor com termo $f_{4}$ não nulo. Assim, já podemos assumir $a$ e $b$ iguais a zero. Assim, basta mostrar que $e_{6}$ não é tangente ao conjunto. De fato, $J \Psi_{1} \cdot e_{6}=\left(F^{\prime}(x), F^{\prime \prime}(x), 0,0\right)=\left(0, F^{\prime \prime}(x), 0,0\right)$, que só é diferente de $(0,0,0,0)$ caso $F^{\prime \prime}(x) \neq 0$.

Um ponto onde $F(x)=0, F^{\prime}(x)=0$ mas $F^{\prime \prime}(x) \neq 0$ é um ponto de sela-nó de codimensão um. Dessa forma, o subconjunto da dobra geral que equivale a $S N_{1}$ é uma superfície imersa em $\mathcal{W}$. Ela é $C^{\infty}$ pois é a projeção sem singularidades de uma superfície $C^{\infty}$. 
Para que $S N_{1}$ seja mergulhada, como $\left[0, \frac{1}{\lambda_{0}}\right]$ é compacto e a dobra geral é fechada, só precisamos que $\Pi$ restrita à dobra geral seja injetora. De fato:

Lema 4.3.3. Sejam $S$ uma superfície com bordo mergulhada e fechada de $K \times \mathbb{R}^{n}$, onde $K$ é um intervalo fechado de $\mathbb{R}$ e $M \subset S$ subconjunto aberto de $S$ sem bordo. Se a restrição da projeção $\Pi: K \times \mathbb{R}^{n} \mapsto \mathbb{R}^{n}$ sobre $S$ for injetora e $\Pi(M)$ for imersa, então $\Pi(M)$ é mergulhada.

Demonstração. Suponha por absurdo que a superfície $\Pi(M)$ não seja mergulhada. Nesse caso, existe $(x, y) \in M$ tal que, não existe vizinhança de $\Pi((x, y))=y$ que não intersecte outras componentes de $\Pi(M)$. Nos referimos aqui as possíveis componentes afastadas na topologia intrínsica mas próximas na topologia induzida. Assim, existe $\left(x_{n}, y_{n}\right) \in S$ tal que $\Pi\left(\left(x_{n}, y_{n}\right)\right)$ converge para $\Pi((x, y))$, mas $\Pi\left(\left(x_{n}, y_{n}\right)\right)$ não pertence à mesma componente de $\Pi(x, y)$.

Assim, $y_{n}$ converge para $y$ e como $\left(x_{n}\right)$ é uma sequência em um conjunto compacto, podemos assumir, sem perda de generalidade, que ela converge para um $x_{0}$. Como $S$ é fechado, e $\left(x_{n}, y_{n}\right)$ converge para $\left(x_{0}, y\right)$, então $\left(x_{0}, y\right) \in S$. Assim, concluímos que $x_{0}=x$ pois $\Pi$ restrita a $S$ é injetora. Mas então $\left(x_{n}, y_{n}\right)$ converge para $(x, y)$ e chegamos a um absurdo uma vez que $S$ é mergulhada.

Agora, devemos tratar da condição de transversalidade para as bifurcações de sela-nó de codimensão um.

Lema 4.3.4. A superfície onde $F(x)=0, F^{\prime}(x)=0$ e $F^{\prime \prime}(x) \neq 0$ corresponde a bifurcações de sela-nó de codimensão um.

Demonstração. As condições necessárias e de não-degenerescência são colocadas como hipóteses. Basta verificar a condição de transversalidade. Para isso a aplicação $(x, \alpha, \beta, \mu, \delta, \lambda) \mapsto$ $\left(F(x), F^{\prime}(x)\right)$ deve ser uma submersão. Mais do que isso, já mostramos (ver lema 4.2.1) que, fixado $\left(\delta_{0}, \lambda_{0}\right)$ a aplicação $(x, \alpha, \beta, \mu) \mapsto\left(F(x), F^{\prime}(x)\right)$ é uma submersão e portanto temos o resultado. 
Notemos, por fim, que quando $F(x), F^{\prime}(x)$ e $F^{\prime \prime}(x)$ se anulam temos uma sela-nó de codimensão dois. Caso esse conjunto seja não vazio (e o é), temos que SN1 será subordinada a ele pela continuidade de $\Psi_{2}$. Além disso, pela própria análise da bifurcação SN2, sabemos que existem pelo menos duas superfícies SN1 subordinadas a uma curva cuspidal SN2.

\subsection{Estudo de $\Psi_{2}$}

A condição necessária para que um ponto seja um candidato a bifurcação sela-nó de codimensão dois é que $F(x), F^{\prime}(x)$ e $F^{\prime \prime}(x)$ se anulem simultaneamente. Assim sendo, o conjunto $\Psi_{2}^{-1}((0,0, \delta, \lambda, 0))$ corresponde a todos os candidatos a bifurcação do tipo sela-nó de codimensão dois. Devemos estudar a função $\Psi_{2}$ restrita a esse conjunto:

Lema 4.4.1. A aplicação $\Psi_{2}$ restrita à $\Psi_{2}^{-1}((0,0, \delta, \lambda, 0))$ é uma submersão quando $F^{\prime \prime \prime}(x) \neq$ 0 .

Demonstração. Comecemos notando que $\Psi_{2}$ é igual a $\left(\Psi_{1}, F^{\prime \prime}(x)\right)$. Além disso $\Psi_{2}^{-1}(0,0, \delta, \lambda, 0) \subseteq$ $\Psi_{1}^{-1}(0,0, \delta, \lambda)$. Logo, já percebemos que o Jacobiano de $\Psi_{2}$ tem pelo menos posto 4 .

Vamos assumir agora que $F^{\prime \prime \prime}(x) \neq 0$. Assim:

$$
J \Psi_{2} \cdot e_{6}=\left(\begin{array}{c}
0 \\
0 \\
0 \\
0 \\
F^{\prime \prime \prime}(x)
\end{array}\right)
$$

que gera $f_{5}$. Agora, pela prova de que $\Psi_{1}$ é uma submersão, temos que existem $A, B, C, D$, $E$ e $G$, números reais, tais que:

$$
\begin{aligned}
& \left\{\begin{array}{l}
\left(A J \Psi_{2} \cdot e_{1}+B J \Psi_{2} \cdot e_{2}+C J \Psi_{2} \cdot e_{3}\right) \cdot f_{1}=1 \\
\left(A J \Psi_{2} \cdot e_{1}+B J \Psi_{2} \cdot e_{2}+C J \Psi_{2} \cdot e_{3}\right) \cdot f_{2}=0
\end{array}\right. \\
& \left\{\begin{array}{l}
\left(D J \Psi_{2} \cdot e_{1}+E J \Psi_{2} \cdot e_{2}+G J \Psi_{2} \cdot e_{3}\right) \cdot f_{1}=0 \\
\left(D J \Psi_{2} \cdot e_{1}+E J \Psi_{2} \cdot e_{2}+G J \Psi_{2} \cdot e_{3}\right) \cdot f_{2}=1
\end{array}\right.
\end{aligned}
$$


Assim sendo, temos que $A J \Psi_{2} \cdot e_{1}+B J \Psi_{2} \cdot e_{2}+C J \Psi_{2} \cdot e_{3}=f_{1}+a f_{5}$ para algum $a \in \mathbb{R}$ e $D J \Psi_{2} \cdot e_{1}+E J \Psi_{2} \cdot e_{2}+G J \Psi_{2} \cdot e_{3}=f_{2}+b f_{6}$ para algum $b \in \mathbb{R}$. No entanto, como $f_{5}$ é gerado por $J \Psi_{2} \cdot e_{6}$, então $\left\{J \Psi_{2} \cdot e_{1}, J \Psi_{2} \cdot e_{2}, J \Psi_{2} \cdot e_{3}, J \Psi_{2} \cdot e_{1}\right\}$ geram $\left\{f_{1}, f_{2}, f_{5}\right\}$. Por fim, como $J \Psi_{2} \cdot e_{4}$ e $J \Psi_{2} \cdot e_{5}$ são os únicos vetores com componentes não nulas em $f_{3}$ e $f_{4}$ respectivamente, temos que $\Psi_{2}$ é uma submersão.

Assim, sempre que $F^{\prime \prime \prime}(x)$ seja diferente de zero, $\Psi_{2}$ é uma submersão. Isso já nos basta, pois, para que uma bifurcação seja de codimensão dois, a condição de não-degenerescência é justamente $F^{\prime \prime \prime}(x) \neq 0$.

\section{5 $S N_{2}$}

Já sabemos que $\Psi_{2}$ restrita ao conjunto $\Psi_{2}^{-1}(0,0, \delta, \lambda, 0)$ é uma submersão caso $F^{\prime \prime \prime}(x) \neq 0$. Fixado $\left(\delta_{0}, \lambda_{0}\right) \in \Delta$, isso significa que a superfície $\Psi_{2}^{-1}\left(0,0, \delta_{0}, \lambda_{0}, 0\right)$ onde $F^{\prime \prime \prime}(x) \neq 0$ é mergulhada em $\mathbb{R} \times \mathcal{W}$.

No entanto, sabemos que $x$ deve ser menor ou igual a $\frac{1}{\lambda_{0}}$ e, portanto, o conjunto que é de nosso interesse é $\left(\Psi_{2}^{-1}\left(0,0, \delta_{0}, \lambda_{0}, 0\right) /\left\{F^{\prime \prime \prime}=0\right\}\right) \cap \mathcal{W} \times\left(\delta_{0}, \lambda_{0}\right) \times\left[0, \frac{1}{\lambda_{0}}\right]$. Esse conjunto é uma curva mergulhada, mas passa a ter bordo em $x=\frac{1}{\lambda_{0}}$. Esse bordo corresponde a bifurcação transcrítica de codimensão três. Agora, podemos fazer uso da projeção П que já foi definida.

O conjunto que é de nosso presente interesse em $\mathcal{W}$ corresponde a $\Pi\left(\left(\Psi_{2}^{-1}\left(0,0, \delta_{0}, \lambda_{0}, 0\right) /\left\{F^{\prime \prime \prime}=\right.\right.\right.$ $\left.0\}) \cap \mathcal{W} \times\left(\delta_{0}, \lambda_{0}\right) \times\left[0, \frac{1}{\lambda_{0}}\right]\right):$

Definição 4.5.1. Fixado $\left(\delta_{0}, \lambda_{0}\right) \in \Delta$, chamaremos de curva de cúspide o conjunto

$$
\Pi\left(\left(\Psi_{2}^{-1}\left(0,0, \delta_{0}, \lambda_{0}, 0\right) /\left\{F^{\prime \prime \prime}=0\right\}\right) \cap \mathcal{W} \times\left(\delta_{0}, \lambda_{0}\right) \times\left[0, \frac{1}{\lambda_{0}}\right]\right)
$$

Lema 4.5.2. Um ponto da curva de cúspide é regular se, e somente se, ele pertence a superfície de sela-nó de codimensão dois $\left(S N_{2}\right)$. Em particular, $S_{2} N_{2}$ é uma curva imersa $C^{\infty}$ de $\mathcal{W}$ 
Demonstração. Tome $\left(\alpha_{0}, \beta_{0}, \mu_{0}\right)=\Pi\left(\alpha_{0}, \beta_{0}, \mu_{0}, \delta_{0}, \lambda_{0}, x_{0}\right)$. Este ponto será regular caso a aplicação $\Pi$ restrita ao espaço tangente da curva $\Psi_{2}^{-1}\left(0,0, \delta_{0}, \lambda_{0}, 0\right)$ em $\left(\alpha_{0}, \beta_{0}, \mu_{0}, \delta_{0}, \lambda_{0}, x_{0}\right)$ seja difeomorfismo. Isso ocorre sempre que a intersecção do subespaço gerado por $e_{4}, e_{5} \mathrm{e}$ $e_{6}$ com o espaço tangente da curva $\Psi_{2}^{-1}\left(0,0, \delta_{0}, \lambda_{0}, 0\right)$ for vazio. Isso é verdade sempre que $F^{\prime \prime \prime}(x) \neq 0$.

De fato, tome um vetor nesse subespaço: $V=a e_{4}+b e_{5}+c e_{6}$. Queremos saber quando $J \Psi_{2} \cdot V \neq(0,0,0,0,0)$. Vamos assumir por absurdo que $J \Psi_{2} \cdot V=(0,0,0,0,0)$. Agora, $J \Psi_{2} \cdot e_{4}$ é o único vetor com termo $f_{3}$ não nulo e $J \Psi_{2} \cdot e_{5}$ é o único vetor com termo $f_{4}$ não nulo. Assim, já podemos assumir $a$ e $b$ iguais a zero. Assim, basta mostrar que $e_{6}$ não é tangente à curva. De fato, $J \Psi_{1} \cdot e_{6}=\left(F^{\prime}(x), F^{\prime \prime}(x), 0,0, F^{\prime \prime \prime}(x)\right)=\left(0,0,0,0, F^{\prime \prime \prime}(x)\right)$, que só é diferente de $(0,0,0,0)$ caso $F^{\prime \prime \prime}(x) \neq 0$.

Um ponto onde $F(x)=0, F^{\prime}(x)=0, F^{\prime \prime}(x)=0$ mas $F^{\prime \prime \prime}(x) \neq 0$ é um ponto de selanó de codimensão dois. Dessa forma, o subconjunto da dobra geral que equivale a $S N_{2}$ é uma curva imersa em $\mathcal{W}$. Ela é $C^{\infty}$ pois é a projeção sem singularidade de uma superfície $C^{\infty}$

Além disso, podemos garantir mais:

Lema 4.5.3. Fixado $\left(\delta_{0}, \lambda_{0}\right)$, caso não exista ponto onde $F(x)=0, F^{\prime}(x)=0, F^{\prime \prime}(x)=0$ e $F^{\prime \prime \prime}(x)=0$, então $S N_{2}$ é mergulhada.

Demonstração. Neste caso, a curva de cúspides passa a ser fechada. Assim, para que a projeção seja um mergulho, basta que $\Pi$ restrita a curva seja injetora. De fato, suponha que não seja verdade. Logo existe $\left(\alpha_{0}, \beta_{0}, \mu_{0}\right)$ e dois pontos $x_{1} \neq x_{2}$ onde $F(x)=0, F^{\prime}(x)=0$, $F^{\prime \prime}(x)=0$. Isso implica que $F$ tem grau pelo menos seis em $x$. Absurdo. Logo $\Pi$ restrito a curva de cúspides é injetora e sua projeção é um mergulho.

Agora, devemos tratar da condição de transversalidade das bifurcações de sela-nó de codimensão dois.

Lema 4.5.4. A superfície onde $F(x)=0, F^{\prime}(x)=0, F^{\prime \prime}(x)=0$ e $F^{\prime \prime \prime}(x) \neq 0$ corresponde a bifurcações de sela-nó de codimensão dois. 
Demonstração. As condições necessárias e de não-degenerescência são colocadas como hipóteses. Basta verificar a condição de transversalidade. Para isso a aplicação $(x, \alpha, \beta, \mu, \delta, \lambda) \mapsto$ $\left(F(x), F^{\prime}(x), F^{\prime \prime}(x)\right)$ deve ser uma submersão. Mais do que isso, já mostramos que, fixado $\left(\delta_{0}, \lambda_{0}\right)$ a aplicação $(x, \alpha, \beta, \mu) \mapsto\left(F(x), F^{\prime}(x), F^{\prime \prime}(x)\right)$ é uma submersão e portanto temos o resultado.

Resta apenas garantir que não temos mais degenerescências.

\subsection{Análise de $\left(F(x), F^{\prime}(x), F^{\prime \prime}(x), F^{\prime \prime \prime}(x)\right)$}

Conjecturamos que a aplicação $(\alpha, \beta, \mu, \delta, \lambda, x) \mapsto\left(F(x), F^{\prime}(x), F^{\prime \prime}(x), F^{\prime \prime \prime}(x)\right)$ nunca se anula para os parâmetros válidos. Nos baseamos em indícios numéricos e em Análise Intervalar (ver $[16])$.

Além disso, provaremos no capítulo 6 que a dobra geral não se intersecta caso $\delta \lambda>\frac{1}{8} \mathrm{e}$ $\beta>0$. Em particular, provaremos que $F(x)$ não possui uma raiz de multiplicidade maior do que três caso $\delta \lambda>\frac{1}{8}$ e $\beta>0$. Dessa forma, provaremos que para certos parâmetros não existe bifurcação do tipo sela-nó de codimensão três

\subsection{Conclusão}

Nesta secção, chegamos no seguinte resultado:

Teorema 4.7.1. Para todo $(\delta, \lambda) \in \Delta$ fixo, o conjunto de bifurcações em $\mathcal{W}$ possui uma superfície de bifurcação do tipo sela-nó subordinada a uma curva de bifurcação do tipo selanó de codimensão dois.

Conjecturamos que não ocorrem bifurcação do tipo sela-nó de codimensão três. 


\section{Capítulo 5}

\section{Estudo sobre as bifurcações de Hopf}

Neste capítulo vamos estudar as bifurcações de Hopf que ocorrem para parâmetros válidos $(\mathcal{W} \times \Delta)$. Para isso, vamos dividir este capítulo em oito seç̧ões: na primeira secção, apresentaremos a estratégia de estudo. Na segunda secção, vamos fazer mudanças na forma normal de forma a calcular os coeficientes de Lyapunov do sistema. Na terceira e quarta secções obteremos informações sobre as superfícies de bifurcações de Hopf de codimensão um, na quinta seç̧ão faremos considerações sobre as bifurcações de Bautin. Na secção seis, discutiremos a possibilidade de haver bifurcações de Hopf mais degeneradas. Por fim, traremos as conclusões finais na sétima secção e a última secção será um apêndice com os cálculos do capítulo.

\section{$5.1 \quad$ Estratégia}

Uma condição necessária para que a bifurcação de Hopf ocorra é que $F(x)$ e o traço do linearizado na singularidade se anulem. No entanto, neste capítulo, temos interesse em analisar as bifurcações de Hopf de codimensão um, dois, ou maiores e não nos preocuparemos com outros tipos de degenerescência (como Bogdanov-Takens por exemplo). Logo, podemos assumir que $F^{\prime}(x) \neq 0$, uma vez que se $F^{\prime}(x)=0$ teriamos uma bifurcação de BogdanovTakens.

Para realizar esse estudo começaremos fazendo uma mudança de variáveis de forma a trazer 
o equilíbrio para a origem. Além disso, seguindo o procedimento padrão para esse tipo de análise (vide [8]) transformaremos o sistema em um sistema complexo:

$$
\dot{z}=(H 1+i \omega) z+H 2 z^{2} \bar{z}+H 3 z^{3} \bar{z}^{2}+\mathcal{O}\left((|z|)^{7}\right)
$$

onde H1, H2 e H3 são os coeficientes de Lyapunov. Uma bifurcação de Hopf ocorre quando $H 1$ se anula e $H 2$ é não nulo, além de respeitar as condições de transversalidade. Uma bifurcação de Hopf de codimensão 2 ocorre quando $H 1$ e $H 2$ se anulam mas $H 3$ é não nulo, além de respeitar as condições de transversalidade. Acreditamos que $H 1, H 2$ e $H 3$ não se anulam simultaneamente e portanto não teremos mais degenerescências.

Para analisar as superfícies de bifurcações usaremos as aplicações: $\mathcal{H}_{1}$ (Hopf de codimensão 1) e $\mathcal{H}_{2}$ (Bautin). Elas são definidas como:

$$
\begin{array}{rlcc}
\mathcal{H}_{1}: \mathcal{W} \times \Delta \times \mathbb{R}^{+} & \rightarrow & \mathbb{R}^{4} \\
(\alpha, \beta, \mu, \delta, \lambda, x) & \mapsto & (F(x), H 1(x), \delta, \lambda) \\
\mathcal{H}_{2}: & \mathcal{W} \times \Delta \times \mathbb{R}^{+} \quad \rightarrow & \mathbb{R}^{5} \\
(\alpha, \beta, \mu, \delta, \lambda, x) & \mapsto & (F(x), H 1(x), \delta, \lambda, H 2(x))
\end{array}
$$

Através dessas aplicações mostraremos a regularidade das superfícies de bifurcação e que elas respeitam as condições de transversalidade.

\subsection{Forma Normal}

Começamos o estudo das bifurcações de Hopf, colocando o sistema na forma normal padrão para esse tipo de análise. Tal forma normal é encontrada no livro [8].

Seja $\left(x_{0}, y_{0}\right)$ com parâmetros $\left(\alpha_{0}, \beta_{0}, \mu_{0}, \delta_{0}, \lambda_{0}\right)$ um equilíbrio no qual o traço do Jacobiano é zero, mas o determinante é positivo. Vamos começar localizando a singularidade na origem:

$$
\begin{aligned}
& u=x-x_{0} \\
& v=y-y_{0}
\end{aligned}
$$


Assim, transformamos o sistema em:

$$
\begin{gathered}
\dot{u}=\left(u+x_{0}\right)\left(1-\lambda\left(u+x_{0}\right)\right)-\frac{\left(v+y_{0}\right)\left(u+x_{0}\right)}{\alpha\left(u+x_{0}\right)^{2}+\beta\left(u+x_{0}\right)+1} \\
\dot{v}=\left(v+y_{0}\right)\left(-\delta-\mu\left(v+y_{0}\right)\right)+\frac{\left(v+y_{0}\right)\left(u+x_{0}\right)}{\alpha\left(u+x_{0}\right)^{2}+\beta\left(u+x_{0}\right)+1}
\end{gathered}
$$

A origem é um equilíbrio onde ocorre uma bifurcação de Hopf para $\left(\alpha_{0}, \beta_{0}, \mu_{0}, \delta_{0}, \lambda_{0}\right)$. Agora vamos considerar uma expansão do sistema:

$$
\begin{gathered}
\dot{u}=c_{0,1} u+c_{0,2} v+n_{1} u^{2}+n_{2} u v+n_{3} v^{2}+n_{4} u^{3}+n_{5} u^{2} v+n_{6} u v^{2}+n_{7} v^{3} \\
+n_{8} u^{4}+n_{9} u^{3} v+n_{10} u^{2} v^{2}+n_{11} u v^{3}+n_{12} v^{4}+n_{13} u^{5} \\
+n_{14} u^{4} v+n_{15} u^{3} v^{2}+n_{16} u^{2} v^{3}+n_{17} u v^{4}+n_{18} v^{5}+\mathcal{O}\left((x+y)^{6}\right) \\
\dot{v}=c_{0,3} u+c_{0,4} v+h_{1} u^{2}+h_{2} u v+h_{3} v^{2}+h_{4} u^{3}+h_{5} u^{2} v+h_{6} u v^{2}+h_{7} v^{3} \\
+h_{8} u^{4}+h_{9} u^{3} v+h_{10} u^{2} v^{2}+h_{11} u v^{3}+h_{12} v^{4}+h_{13} u^{5} \\
+h_{14} u^{4} v+h_{15} u^{3} v^{2}+h_{16} u^{2} v^{3}+h_{17} u v^{4}+h_{18} v^{5}+\mathcal{O}\left((x+y)^{6}\right)
\end{gathered}
$$

onde os valores desses termos estão no apêndice deste capítulo. Feita essa transformação, notemos que o valor de $c_{0,2}=-\frac{x_{0}}{\alpha x_{0}^{2}+1+\beta x_{0}}$ é sempre diferente de zero, pois $x_{0}$ não se encontra na origem, e que a equação dos autovalores do linearizado é dado por:

$$
\Lambda_{+-}=1 / 2 c_{0,4}+1 / 2 c_{0,1}+-\sqrt{c_{0,1}^{2}-2 c_{0,1} c_{0,4}+c_{0,4}^{2}+4 c_{0,2} c_{0,3}}
$$

assim, assumir que estamos sobre um equilíbrio com autovalores complexos, significa que $-\left(c_{0,1}^{2}-2 c_{0,1} c_{0,4}+c_{0,4}^{2}+4 c_{0,2} c_{0,3}\right)>0$. Dessa forma, podemos fazer a seguinte mudança de variáveis:

$$
\begin{gathered}
x=u \\
y=\frac{\left(c_{0,4}-c_{0,1}\right) u-2 c_{0,2} v}{\sqrt{-\left(c_{0,1}^{2}-2 c_{0,1} c_{0,4}+c_{0,4}{ }^{2}+4 c_{0,2} c_{0,3}\right)}}
\end{gathered}
$$

Com essa mudança de coordenadas obtemos um sistema cuja linearização calculada na origem vale:

$$
\left[\begin{array}{cc}
1 / 2 c_{0,4}+1 / 2 c_{0,1} & -1 / 2 \sqrt{-c_{0,1}{ }^{2}+2 c_{0,1} c_{0,4}-c_{0,4}{ }^{2}-4 c_{0,2} c_{0,3}} \\
1 / 2 \sqrt{-c_{0,1}{ }^{2}+2 c_{0,1} c_{0,4}-c_{0,4}{ }^{2}-4 c_{0,2} c_{0,3}} & 1 / 2 c_{0,4}+1 / 2 c_{0,1}
\end{array}\right]
$$


Assim, obtemos um sistema do tipo:

$$
\begin{gathered}
\dot{x}=\quad \text { Rn } x-\text { In } y+c_{1} x^{2}+c_{2} x y+c_{3} y^{2}+c_{4} x^{3}+c_{5} x^{2} y+c_{6} x y^{2}+c_{7} y^{3} \\
+c_{8} x^{4}+c_{9} x^{3} y+c_{10} x^{2} y^{2}+c_{11} x y^{3}+c_{12} y^{4}+c_{13} x^{5} \\
+c_{14} x^{4} y+c_{15} x^{3} y^{2}+c_{16} x^{2} y^{3}+c_{17} x y^{4}+c_{18} y^{5}+\mathcal{O}\left((x+y)^{6}\right) \\
\dot{y}=\quad \text { inx }+ \text { Rny }+d_{1} x^{2}+d_{2} x y+d_{3} y^{2}+d_{4} x^{3}+d_{5} x^{2} y+d_{6} x y^{2}+d_{7} y^{3} \\
+d_{8} x^{4}+d_{9} x^{3} y+d_{10} x^{2} y^{2}+d_{11} x y^{3}+d_{12} y^{4}+d_{13} x^{5} \\
+d_{14} x^{4} y+d_{15} x^{3} y^{2}+d_{16} x^{2} y^{3}+d_{17} x y^{4}+d_{18} y^{5}+\mathcal{O}\left((x+y)^{6}\right)
\end{gathered}
$$

onde $R n=1 / 2 c_{0,4}+1 / 2 c_{0,1}$ e $I n=1 / 2 \sqrt{-c_{0,1}{ }^{2}+2 c_{0,1} c_{0,4}-c_{0,4}{ }^{2}-4 c_{0,2} c_{0,3}}$. As expressões dos demais termos encontram-se no apêndice desse capítulo. Já estamos em condições de passar para variáveis complexas. Tomando $z=x+i y$ temos que:

$$
\dot{z}=(R n+i I N) z+\sum_{k+l>1}^{n} \frac{1}{k ! l !} g_{k l} z^{k} \bar{z}^{l}+\mathcal{O}\left((|z|)^{n+1}\right)
$$

No apêndice deste capítulo temos as expressões dos $g_{k l}$ para $K+l \leq 5$.

Ao colocarmos o sistema nessa forma, podemos usar as formulas encontradas no livro [8] para obter:

$$
\dot{z}=(H 1+i \omega) z+H 2 z^{2} \bar{z}+H 3 z^{3} \bar{z}^{2}+\mathcal{O}\left((|z|)^{7}\right)
$$

onde $H 1$ é igual ao traço do linearizado calculado no equilíbrio. Dessa forma:

$$
H 1\left(x_{0}, y_{0}, \alpha_{0}, \beta_{0}, \mu_{0}, \delta_{0}, \lambda_{0}\right)=0
$$

pela hipótese de estarmos trabalhando sobre um ponto no qual o traço vale zero.

\subsection{Estudo de $\mathcal{H}_{1}$}

Lembremos da aplicação $\mathcal{H}_{1}$ :

$$
\begin{array}{rlcc}
\mathcal{H}_{1}: & \mathcal{W} \times \Delta \times \mathbb{R}^{+} & \rightarrow & \mathbb{R}^{4} \\
(\alpha, \beta, \mu, \delta, \lambda, x) & \mapsto & (F(x), H 1(x), \delta, \lambda)
\end{array}
$$


onde

$$
\begin{gathered}
H 1(x)=\mu \lambda x^{5} \alpha^{2}+\left(-\mu \alpha^{2}+2 \mu \lambda \alpha \beta\right) x^{4}+\left(2 \mu \lambda \alpha-2 \mu \alpha \beta-3 \lambda \alpha+\mu \lambda \beta^{2}\right) x^{3} \\
+\left(2 \alpha-2 \lambda \beta-2 \mu \alpha-\mu \beta^{2}+2 \mu \lambda \beta\right) x^{2}+(\mu \lambda-2 \mu \beta+\beta-\lambda) x-\mu
\end{gathered}
$$

Sabemos que uma condição necessária para uma bifurcação de Hopf ocorrer é $F(x)=0$ e $H 1(x)=0$. Assim, $\mathcal{H}_{1}^{-1}(0,0, \delta, \lambda)$ é o conjunto de candidatos a bifurcação de Hopf.

Lema 5.3.1. A aplicação $\mathcal{H} 1$ é uma submersão quando restrita ao conjunto $\mathcal{H} 1^{-1}(0,0, \delta, \lambda)$.

Demonstração. Tomemos o jacobiano de $\mathcal{H} 1$ que chamaremos de $J H 1$, a base canônica $\left\{e_{1}\right.$, $\left.e_{2}, \ldots, e_{6}\right\}$ do $\mathbb{R}^{6}$ e a base canônica $\left\{f_{1}, f_{2}, f_{3}, f_{4}\right\}$ do $\mathbb{R}^{4}$. Assim, temos que:

$$
\begin{gathered}
J H 1 \cdot e_{1}=\left[\begin{array}{c}
-\delta x^{2}+2 \mu(-1+\lambda x)\left(\alpha x^{2}+\beta x+1\right) x^{2} \\
2 \mu \lambda x^{5} \alpha+(-2 \mu \alpha+2 \mu \lambda \beta) x^{4}+(2 \mu \lambda-2 \mu \beta-3 \lambda) x^{3}+(2-2 \mu) x^{2} \\
0 \\
0
\end{array}\right] \\
J H 1 \cdot e_{2}=\left[\begin{array}{c}
-\delta x+2 \mu(-1+\lambda x)\left(\alpha x^{2}+\beta x+1\right) x \\
2 \mu \lambda \alpha x^{4}+(-2 \mu \alpha+2 \mu \lambda \beta) x^{3}+(-2 \lambda-2 \mu \beta+2 \mu \lambda) x^{2}+(-2 \mu+1) x \\
0 \\
0 \\
J H 1 \cdot e_{3}=\left[\begin{array}{c}
(-1+\lambda x)\left(\alpha x^{2}+\beta x+1\right)^{2} \\
\lambda x^{5} \alpha^{2}+\left(-\alpha^{2}+2 \lambda \alpha \beta\right) x^{4}+\left(2 \lambda \alpha-2 \beta \alpha+\lambda \beta^{2}\right) x^{3}+ \\
+\left(-2 \alpha-\beta^{2}+2 \lambda \beta\right) x^{2}+(\lambda-2 \beta) x-1 \\
0
\end{array}\right]
\end{array}\right]
\end{gathered}
$$




$$
J H 1 \cdot e_{4}=\left(\begin{array}{c}
-\left(\alpha x^{2}+\beta x+1\right) \\
0 \\
1 \\
0
\end{array}\right) e_{5}=\left[\begin{array}{c}
\mu x\left(\alpha x^{2}+\beta x+1\right)^{2} \\
\mu x^{5} \alpha^{2}+2 \mu \alpha \beta x^{4}+\left(2 \mu \alpha-3 \alpha+\mu \beta^{2}\right) x^{3}+(-2 \beta+2 \mu \beta) x^{2}+(\mu-1) x \\
0 \\
1 \\
F^{\prime}(x) \\
H 1^{\prime}(x) \\
0 \\
0
\end{array}\right)
$$

Note que apenas em $J H 1 \cdot e_{4}$ o termo relativo a $f_{3}$ é diferente de zero. A mesma coisa acontece em $J H 1 \cdot e_{5}$ em relação à $f_{4}$. Assim, para que a aplicação seja sobrejetiva, basta que $J H 1 \cdot e_{1}, J H 1 \cdot e_{2}, J H 1 \cdot e_{3}$ e $J H 1 \cdot e_{6}$ gerem o subespaço $\left\{f_{1}, f_{2}\right\}$. Na verdade, apenas os vetores $\left\{J H 1 \cdot e_{1}, J H 1 \cdot e_{2}, J H 1 \cdot e_{3}\right\}$ já serão suficientes. De fato, como

$$
0=F(x)=-\delta\left(\alpha x^{2}+\beta x+1\right)+\mu(-1+\lambda x)\left(\alpha x^{2}+\beta x+1\right)^{2}+x
$$

então:

$$
2 \mu(-1+\lambda x)\left(\alpha x^{2}+\beta x+1\right)=2 \frac{-x}{\left(\alpha x^{2}+\beta x+1\right)}+2 \delta
$$

Dessa forma:

$$
\begin{aligned}
& J H 1 \cdot e_{1}=\left(\begin{array}{c}
x^{2}\left(\frac{-2 x}{\alpha x^{2}+\beta x+1}+\delta\right) \\
x E q 1 \\
0 \\
0
\end{array}\right) \\
& J H 1 \cdot e_{2}=\left(\begin{array}{c}
x\left(\frac{-2 x}{\alpha x^{2}+\beta x+1}+\delta\right) \\
E q 1+x(\lambda x-1) \\
0 \\
0
\end{array}\right)
\end{aligned}
$$


Aqui, já estamos em condições de considerar um caso especifico. Suponha que $\frac{-2 x}{\alpha x^{2}+\beta x+1}+\delta \neq$ 0. Afirmo que esses dois vetores já são suficientes. De fato, note que esses vetores estão contidos no subespaço $\left\{f_{1}, f_{2}\right\}$. Assim, vamos nos restringir as coordenadas referentes a esse subespaço. Passamos a ter dois vetores com duas entradas. Para mostrar que eles são LI's e que, assim, geram o subespaço $\left\{f_{1}, f_{2}\right\}$ basta tomar o determinante da restrição desses vetores. O que nos gera:

$$
x^{3}(\lambda x-1)\left(\frac{-2 x}{\alpha x^{2}+\beta x+1}+\delta\right)
$$

Como $x \neq 0$ pois a única singularidade quando $x=0$ é uma sela, como $x \neq \frac{1}{\lambda}$ pois a única singularidade nesse ponto é quando $y=0$ e portanto estamos no ponto analisado na bifurcação transcrítica que em nenhum momento passa por uma bifurcação de Hopf, temos que o determinante é diferente de zero e portanto esses dois vetores geram o subespaço $\left\{f_{1}, f_{2}\right\}$. Agora vamos tratar o caso em que $\frac{-2 x}{\alpha x^{2}+\beta x+1}+\delta=0$

Assumindo também que $\mu \neq 0$, como

$$
0=F(x)=-\delta\left(\alpha x^{2}+\beta x+1\right)+\mu(-1+\lambda x)\left(\alpha x^{2}+\beta x+1\right)^{2}+x
$$

temos que:

$$
(-1+\lambda x)\left(\alpha x^{2}+\beta x+1\right)^{2}=\frac{1}{\mu}\left(\delta\left(\alpha x^{2}+\beta x+1\right)-x\right)
$$

Além disso, como:

$$
\begin{gathered}
H 1=0=\left(\lambda x^{5} \alpha^{2}+\left(-\alpha^{2}+2 \lambda \alpha \beta\right) x^{4}+\left(2 \lambda \alpha-2 \beta \alpha+\lambda \beta^{2}\right) x^{3}+\left(-2 \alpha-\beta^{2}+2 \lambda \beta\right) x^{2}\right. \\
+(\lambda-2 \beta) x-1) \mu+(2 \alpha-2 \lambda \beta) x^{2}-3 \lambda x^{3} \alpha+(\beta-\lambda) x
\end{gathered}
$$

então:

$$
\begin{gathered}
\lambda x^{5} \alpha^{2}+\left(-\alpha^{2}+2 \lambda \alpha \beta\right) x^{4}+\left(2 \lambda \alpha-2 \beta \alpha+\lambda \beta^{2}\right) x^{3}+\left(-2 \alpha-\beta^{2}+2 \lambda \beta\right) x^{2}+(\lambda-2 \beta) x-1 \\
=-\frac{1}{\mu}\left((2 \alpha-2 \lambda \beta) x^{2}-3 \lambda x^{3} \alpha+(\beta-\lambda) x\right)
\end{gathered}
$$

Assim:

$$
J H 1 \cdot e_{3}=\left(\begin{array}{c}
\frac{1}{\mu}\left(-x+\delta\left(\alpha x^{2}+\beta x+1\right)\right) \\
\frac{1}{\mu}\left(3 \lambda \alpha x^{3}-(2 \alpha-2 \lambda \beta) x^{2}-(\beta-\lambda) x\right) \\
0 \\
0
\end{array}\right)
$$


logo o primeiro termo deste vetor é sempre diferente de zero. Além disso, notemos que o segundo termo dos vetores $J H 1 \cdot e_{1}$ e $J H 1 \cdot e_{2}$ não zeram simultaneamente já que, caso $E q 1=0$, então o segundo termo de $J H 1 \cdot e_{2}$ vale $x(\lambda x-1)$ que é diferente de zero por motivos já discutidos. Assim sendo, o vetor $f_{2}$ pode ser gerado por uma combinação de $J H 1 \cdot e_{1}$ e $J H 1 \cdot e_{2}$. Por fim, como o primeiro termo de $J H 1 \cdot e_{3}$ não zera, temos que $J H 1 \cdot e_{3}=A f_{1}+B f_{2}$, com $A \neq 0$. Além disso, como existe $C, D \in \mathbb{R}$ tais que $C J H 1 \cdot e_{1}+D J H 1 \cdot e_{2}=f_{2}$, temos que: $f_{1}=\frac{J H 1 \cdot e_{3}}{A}-\frac{B\left(C J H 1 \cdot e_{1}+D J H 1 \cdot e_{2}\right)}{A}$ e, portanto, $\mathcal{H}_{1}$ é uma submersão.

Assim, resta o caso em que $\mu=0$ e $\frac{-2 x}{\alpha x^{2}+\beta x+1}+\delta=0$. Mas este caso é facilmente descartado com uma análise de $F(x)$, uma vez que, substituindo $\mu=0$ em $F$ obtemos:

$$
-\delta\left(\alpha x^{2}+\beta x+1\right)+x
$$

que é diferente de zero uma vez que $\frac{-2 x}{\alpha x^{2}+\beta x+1}+\delta=0$ e $x \neq 0$. Logo $\mathcal{H}_{1}$ é uma submersão quando restrita ao conjunto $\mathcal{H}_{1}^{-1}(0,0, \delta, \lambda)$.

\subsection{Superfície $H_{1}$}

Já sabemos que $\mathcal{H}_{1}$ restrita ao conjunto $\mathcal{H}_{1}^{-1}(0,0, \delta, \lambda)$ é uma submersão. Fixado $\left(\delta_{0}, \lambda_{0}\right) \in \Delta$, isso significa que a superfície $\mathcal{H}_{1}^{-1}\left(0,0, \delta_{0}, \lambda_{0}\right)$ é mergulhada e fechada em $\mathcal{W} \times \mathbb{R}$.

Fixado $\left(\delta_{0}, \lambda_{0}\right) \in \Delta$, para que esta superfície tenha interesse para o nosso caso, temos que considerar duas restrições:

- $0 \leq x \leq \frac{1}{\lambda_{0}}$

- o determinante do linearizado do sistema deve ser positivo;

Dessa forma, vamos tomar a intersecção da superfície $\mathcal{H}_{1}^{-1}\left(0,0, \delta_{0}, \lambda_{0}\right) \operatorname{com} \mathcal{W} \times\left(\delta_{0}, \lambda_{0}\right) \times$ $\left[0, \frac{1}{\lambda_{0}}\right]$ e com $\operatorname{Det}^{-1}(] 0, \infty[)$, onde Det é a função determinante do linearizado do sistema. Chamaremos essa superfície de $S$. 
Agora queremos projetar $S$ em $\mathcal{W}$. Para isso, lembremos da seguinte projeção definida no capítulo anterior:

$$
\begin{aligned}
\Pi: \mathcal{W} \times \Delta \times \mathbb{R}_{+} & \rightarrow \mathcal{W} \\
(\alpha, \beta, \mu, \delta, \lambda, x) & \mapsto(\alpha, \beta, \mu)
\end{aligned}
$$

Lema 5.4.1. A superfície $\Pi(S)$ é imersa.

Demonstração. Para $\Pi(y)$ ser regular, basta que a diferencial de $\Pi$ restrita ao plano tangente a $S$ no ponto y seja um difeomorfismo. Usando argumento análogo ao apresentado no capítulo 4, chegamos a conclusão que basta que $J \mathcal{H}_{1} \cdot e_{6} \neq 0$ quando estivermos sobre $\Pi(S)$. Assim, devemos ter:

$$
0 \neq J \mathcal{H}_{1} \cdot e_{6}=\left(\begin{array}{c}
F^{\prime}(x) \\
H 1^{\prime}(x) \\
0 \\
0
\end{array}\right)
$$

Sempre que $F(x)=0=H 1(x)$. Isto é sempre verdade, já que $F^{\prime}(x)=0$ implica que o determinante do linearizado é igual a zero.

Assim, definimos $H_{1}$ como sendo igual a superfície $\Pi(S)$ onde $H 2(x) \neq 0$. Para que esta superfície seja constituida de bifurcações de Hopf, resta mostrar a condição de transversalidade:

Lema 5.4.2. A superfície $H_{1}$ é constituída de bifurcações de Hopf de codimensão um.

Demonstração. As condições necessárias e de não-degenerescência são verificadas por hipóteses. Basta verificar a condição de transversalidade. Para isso a aplicação $(x, \alpha, \beta, \mu, \delta, \lambda) \mapsto$ $(F(x), H 1(x))$ deve ser uma submersão. Mais do que isso, já mostramos (ver lema 5.3.1) que, fixado $\left(\delta_{0}, \lambda_{0}\right)$ a aplicação $(x, \alpha, \beta, \mu) \mapsto(F(x), H 1(x))$ é uma submersão e portanto temos o resultado.

\subsection{Conjectura sobre a curva $H_{2}$}

Gostaríamos de prosseguir com a análise da mesma forma que fizemos para $H_{1}$ e no caso da sela-nó de codimensão dois. No entanto, continuar esse estudo esbarra na difícil missão de 
trabalhar com o coeficiente H2. Tal função possui muitos termos com grau alto, e, portanto, um tratamento algébrico ou analítico não é uma tarefa fácil. De todo modo, já sabemos que a diferencial da função $\mathcal{H}_{2}$ tem posto, no mínimo quatro. Através de uma análise computacional, obtemos indícios numéricos de que esta aplicação seja uma submersão sobre o conjunto $\mathcal{H}_{2}^{-1}(0,0, \delta, \lambda, 0)$.

Assumindo essa conjectura, podemos obter mais informações sobre a curva de bifurcações de Hopf de codimensão dois (Bautin). Primeiro, sabemos que o conjunto $\mathcal{H}_{2}^{-1}(0,0, \delta, \lambda, 0)$ é uma curva mergulhada em $\mathcal{W} \times \mathbb{R}$. Além disso, sabemos que devemos submeter essa curvas as mesmas restrições que impusemos a $H_{1}$ :

- $0 \leq x \leq \frac{1}{\lambda_{0}}$

- o determinante do linearizado do sistema deve ser positivo;

Apenas então podemos projeta-la em $\mathcal{W}$ e retirar o subconjunto relativo a $H 3(x)=0$. Esta curva chamaremos de $H_{2}$. Prosseguindo de forma análoga a $H_{1}$ podemos mostrar que:

- $\mathrm{H}_{2}$ é uma curva imersa em $\mathcal{W}$.

- $\mathrm{H}_{2}$ é constiuída de pontos de bifurcação de Bautin

De fato, analogamente ao lema 4.3.3, para mostrar a primeira afirmação basta que essa curva nunca tenha como vetor tangente o vetor $e_{6}$. Equivalentemente, devemos ter $J \mathcal{H}_{2} \cdot e_{6} \neq 0$ quando estivermos sobre $\Pi\left(\mathcal{H}_{2}^{-1}\left(0,0, \delta_{0}, \lambda_{0}, 0\right)\right)$. Assim, devemos ter:

$$
0 \neq J \mathcal{H}_{1} \cdot e_{6}=\left(\begin{array}{c}
F^{\prime}(x) \\
H 1^{\prime}(x) \\
0 \\
0 \\
H_{2}^{\prime}(x)
\end{array}\right)
$$

Sempre que $F(x)=0=H 1(x)=H 2(x)$. Como estamos sobre um ponto no qual o determinante do linearizado é positivo, temos que $F^{\prime}(x) \neq 0$ e, portanto, $J \mathcal{H}_{2} \cdot e_{6} \neq(0,0,0,0,0)$. 
Resta tratar da condição de transversalidade. Para que o conjunto $H_{2}$ possa ser caracterizado como uma bifurcação de Hopf, a aplicação $(x, \alpha, \beta, \mu, \delta, \lambda) \mapsto(F(x), H 1(x), H 2(x))$ deve ser uma submersão quando restrita a $H_{2}$. Mas isso é equivalente a conjectura que estamos assumindo.

Assim, aceitando a conjectura, acreditamos que exista uma curva de Bautin em $\mathcal{W}$. Resta tratar de possíveis bifurcações mais degeneradas.

\subsection{Conjectura sobre bifurcações de Hopf mais dege- neradas}

Se trabalhar algebricamente com o coeficiente $H 2$ era complicado, trabalhar com o coeficiente $H 3$, mesmo numericamente, é ainda mais complicado. Este coeficiente possui muitos termos, e os métodos de simplificação que utilizamos não foram suficientes para que sua expressão fosse trabalhável algebricamente.

Desta forma, tratamos do problema de forma puramente computacional. Através de uma análise numérica obtemos indícios de que não existem bifurcação de Hopf de codimensão três. Assim, conjecturamos que este seja o caso.

\subsection{Conclusões}

O principal resultado do capítulo é:

Teorema 5.7.1. Para todo $(\delta, \lambda) \in \Delta$ fixo, o conjunto de bifurcações em $\mathcal{W}$ possui uma superfície de bifurcação do tipo Hopf.

Além disso conjecturamos que:

- Para todo $(\delta, \lambda) \in \Delta$ fixo, o conjunto de bifurcações em $\mathcal{W}$ possui uma curva de bifurcação do tipo Bautin. 
- Não ocorrem bifurcações de Hopf de codimensão maior do que dois.

\subsection{Apêndice - Valores dos coeficientes}

Temos, para a primeira transformação os seguintes coeficientes:

$$
\begin{aligned}
& c_{0,1}=\quad 1-2 \lambda x_{0}-\frac{y_{0}}{\alpha x_{0}^{2}+1+\beta x_{0}}+\frac{y_{0} x_{0}\left(\beta+2 \alpha x_{0}\right)}{\left(\alpha x_{0}^{2}+1+\beta x_{0}\right)^{2}} \\
& c_{0,2}=\quad-\frac{x_{0}}{\alpha x_{0}^{2}+1+\beta x_{0}} \\
& c_{0,3}=\quad \frac{y_{0}}{\alpha x_{0}^{2}+1+\beta x_{0}}-\frac{y_{0} x_{0}\left(\beta+2 \alpha x_{0}\right)}{\left(\alpha x_{0}^{2}+1+\beta x_{0}\right)^{2}} \\
& c_{0,4}=\quad-2 \mu y_{0}-\delta+\frac{x_{0}}{\alpha x_{0}^{2}+1+\beta x_{0}} \\
& n_{1}=\quad-\lambda+\frac{y_{0}\left(\beta+2 \alpha x_{0}\right)}{\left(\alpha x_{0}^{2}+1+\beta x_{0}\right)^{2}}-\frac{y_{0} x_{0}\left(\beta+2 \alpha x_{0}\right)^{2}}{\left(\alpha x_{0}^{2}+1+\beta x_{0}\right)^{3}}+\frac{y_{0} x_{0} \alpha}{\left(\alpha x_{0}^{2}+1+\beta x_{0}\right)^{2}} \\
& n_{2}=\quad \frac{y_{0}\left(-\beta+\alpha^{2} x_{0}{ }^{3}-3 \alpha x_{0}\right)}{\left(\alpha x_{0}{ }^{2}+1+\beta x_{0}\right)^{3}} \\
& n_{3}=\quad 0 \\
& n_{4}=\quad \frac{y_{0}\left(-\beta^{2}-4 \beta \alpha x_{0}+\alpha^{3} x_{0}{ }^{4}-6 \alpha^{2} x_{0}{ }^{2}+\alpha\right)}{\left(\alpha x_{0}{ }^{2}+1+\beta x_{0}\right)^{4}} \\
& n_{5}=\quad-\frac{-\beta+\alpha^{2} x_{0}{ }^{3}-3 \alpha x_{0}}{\left(\alpha x_{0}{ }^{2}+1+\beta x_{0}\right)^{3}} \\
& n_{6}=\quad 0 \\
& n_{7}=\quad 0 \\
& n_{8}=\quad-\frac{y_{0}\left(-\beta^{3}-10 \beta \alpha^{2} x_{0}{ }^{2}-5 \beta^{2} \alpha x_{0}-10 \alpha^{3} x_{0}{ }^{3}+\alpha^{4} x_{0}{ }^{5}+2 \alpha \beta+5 \alpha^{2} x_{0}\right)}{\left(\alpha x_{0}{ }^{2}+1+\beta x_{0}\right)^{5}} \\
& n_{9}=\quad \frac{-\beta^{2}-4 \beta \alpha x_{0}+\alpha^{3} x_{0}{ }^{4}-6 \alpha^{2} x_{0}{ }^{2}+\alpha}{\left(\alpha x_{0}{ }^{2}+1+\beta x_{0}\right)^{4}} \\
& n_{10}=\quad 0 \\
& n_{11}=\quad 0 \\
& n_{12}=\quad 0 \\
& n_{13}=\frac{y_{0}\left(-\alpha^{2}-\beta^{4}-15 \alpha^{4} x_{0}{ }^{4}+\alpha^{5} x_{0}{ }^{6}+3 \alpha \beta^{2}+15 \alpha^{3} x_{0}{ }^{2}-20 \beta \alpha^{3} x_{0}{ }^{3}-6 \beta^{3} \alpha x_{0}-15 \beta^{2} \alpha^{2} x_{0}{ }^{2}+12 \beta \alpha^{2} x_{0}\right)}{\left(\alpha x_{0}{ }^{2}+1+\beta x_{0}\right)^{6}} \\
& n_{14}=\quad-\frac{-\beta^{3}-10 \beta \alpha^{2} x_{0}^{2}-5 \beta^{2} \alpha x_{0}-10 \alpha^{3} x_{0}^{3}+\alpha^{4} x_{0}{ }^{5}+2 \alpha \beta+5 \alpha^{2} x_{0}}{\left(\alpha x_{0}{ }^{2}+1+\beta x_{0}\right)^{5}} \\
& n_{15}=\quad 0 \\
& n_{16}=\quad 0 \\
& n_{17}=\quad 0 \\
& n_{18}=\quad 0
\end{aligned}
$$




\begin{tabular}{|c|c|}
\hline $\begin{array}{l}h_{1}= \\
h_{2}=\end{array}$ & $\begin{array}{c}\frac{y_{0}\left(-\beta+\alpha^{2} x_{0}^{3}-3 \alpha x_{0}\right)}{\left(\alpha x_{0}^{2}+1+\beta x_{0}\right)^{3}} \\
-\frac{\alpha x_{0}^{2}-1}{\left(\alpha x_{0}^{2}+1+\beta x_{0}\right)^{2}}\end{array}$ \\
\hline$h_{3}=$ & $-\mu$ \\
\hline$h_{4}=$ & $-\frac{y_{0}\left(-\beta^{2}-4 \beta \alpha x_{0}+\alpha^{3} x_{0}{ }^{4}-6 \alpha^{2} x_{0}^{2}+\alpha\right)}{\left(\alpha x_{0}^{2}+1+\beta x_{0}\right)^{4}}$ \\
\hline$h_{5}=$ & $\frac{-\beta+\alpha^{2} x_{0}^{3}-3 \alpha x_{0}}{\left(\alpha x_{0}^{2}+1+\beta x_{0}\right)^{3}}$ \\
\hline$h_{6}=$ & 0 \\
\hline$h_{7}=$ & 0 \\
\hline$h_{8}=$ & $\frac{y_{0}\left(-\beta^{3}-10 \beta \alpha^{2} x_{0}^{2}-5 \beta^{2} \alpha x_{0}-10 \alpha^{3} x_{0}^{3}+\alpha^{4} x_{0}^{5}+2 \alpha \beta+5 \alpha^{2} x_{0}\right)}{\left(\alpha x_{0}^{2}+1+\beta x_{0}\right)^{5}}$ \\
\hline$h_{9}=$ & $-\frac{-\beta^{2}-4 \beta \alpha x_{0}+\alpha^{3} x_{0}^{4}-6 \alpha^{2} x_{0}^{2}+\alpha}{\left(\alpha x_{0}^{2}+1+\beta x_{0}\right)^{4}}$ \\
\hline$h_{10}=$ & 0 \\
\hline$h_{11}=$ & 0 \\
\hline$h_{12}=$ & 0 \\
\hline & 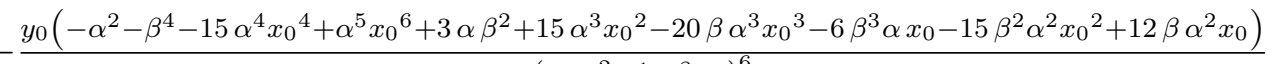 \\
\hline$h_{14}=$ & $\begin{array}{c}\left(\alpha x_{0}^{2}+1+\beta x_{0}\right)^{6} \\
\frac{-\beta^{3}-10 \beta \alpha^{2} x_{0}{ }^{2}-5 \beta^{2} \alpha x_{0}-10 \alpha^{3} x_{0}{ }^{3}+\alpha^{4} x_{0}{ }^{5}+2 \alpha \beta+5 \alpha^{2} x_{0}}{\left(\alpha x_{0}{ }^{2}+1+\beta x_{0}\right)^{5}}\end{array}$ \\
\hline$h_{15}=$ & 0 \\
\hline$h_{16}=$ & 0 \\
\hline$h_{17}=$ & 0 \\
\hline$h_{18}=$ & 0 \\
\hline
\end{tabular}


Na segunda transformação, já zerando os termos que $n$ e $h$ que zeraram no cálculo anterior temos:

$$
\begin{array}{lc}
c_{1}= & -\frac{-n_{1} c_{0,2}+1 / 2 n_{2} c_{0,1}-1 / 2 n_{2} c_{0,4}}{c_{0,2}} \\
c_{2}= & -1 / 2 \frac{n_{2} \sqrt{-c_{0,1}{ }^{2}+2 c_{0,1} c_{0,4}-c_{0,4}{ }^{2}-4 c_{0,2} c_{0,3}}}{c_{0,2}} \\
c_{3}= & 0 \\
c_{4}= & -\frac{-n_{4} c_{0,2}+1 / 2 n_{5} c_{0,1}-1 / 2 n_{5} c_{0,4}}{c_{0,2}} \\
c_{5}= & -1 / 2 \frac{n_{5} \sqrt{-c_{0,1}{ }^{2}+2 c_{0,1} c_{0,4}-c_{0,4}{ }^{2}-4 c_{0,2} c_{0,3}}}{c_{0,2}} \\
c_{6}= & 0 \\
c_{7}= & 0 \\
c_{8}= & -\frac{-n_{8} c_{0,2}+1 / 2 n_{9} c_{0,1}-1 / 2 n_{9} c_{0,4}}{c_{0,2}} \\
c_{9}= & -1 / 2 \frac{n_{9} \sqrt{-c_{0,1}{ }^{2}+2 c_{0,1} c_{0,4}-c_{0,4}{ }^{2}-4 c_{0,2} c_{0,3}}}{c_{0,2}} \\
c_{10}= & 0 \\
c_{11}= & 0 \\
c_{12}= & 0 \\
c_{13}= & -\frac{-n_{13} c_{0,2}+1 / 2 n_{14} c_{0,1}-1 / 2 n_{14} c_{0,4}}{c_{0,2}} \\
c_{14}= & -1 / 2 \frac{n_{14} \sqrt{-c_{0,1}{ }^{2}+2 c_{0,1} c_{0,4}-c_{0,4}{ }^{2}-4 c_{0,2} c_{0,3}}}{c_{0,2}} \\
c_{15}= & 0 \\
c_{16}= & 0 \\
c_{17}= & 0 \\
c_{18}= & 0
\end{array}
$$




$$
\begin{aligned}
& d_{1}=-\left(h_{2} c_{0,2} c_{0,4}-1 / 2 n_{2} c_{0,4}^{2}-1 / 2 n_{2} c_{0,1}^{2}-c_{0,4} n_{1} c_{0,2}-h_{2} c_{0,2} c_{0,1}+1 / 2 h_{3} c_{0,4}{ }^{2}\right. \\
& \left.-h_{3} c_{0,1} c_{0,4}+1 / 2 h_{3} c_{0,1}^{2}+2 h_{1} c_{0,2}^{2}+c_{0,1} n_{2} c_{0,4}+c_{0,1} n_{1} c_{0,2}\right) \\
& \left(c_{0,2} \sqrt{-c_{0,1}^{2}+2 c_{0,1} c_{0,4}-c_{0,4}^{2}-4 c_{0,2} c_{0,3}}\right)^{-1} \\
& d_{2}=\quad-\frac{-1 / 2 n_{2} c_{0,1}-h_{3} c_{0,4}-h_{2} c_{0,2}+1 / 2 n_{2} c_{0,4}+h_{3} c_{0,1}}{c_{0,2}} \\
& d_{3}=\quad \frac{-h_{3} c_{0,1} c_{0,4}+2 h_{3} c_{0,2} c_{0,3}+1 / 2 h_{3} c_{0,1}{ }^{2}+1 / 2 h_{3} c_{0,4}{ }^{2}}{c_{0,2} \sqrt{-c_{0,1}^{2}+2 c_{0,1}}} \\
& d_{4}=\frac{1 / 2 n_{5} c_{0,4}{ }^{2}+1 / 2 n_{5} c_{0,1}^{2}+c_{0,4} n_{4} c_{0,2}-c_{0,1} n_{5} c_{0,4}-c_{0,1} n_{4} c_{0,2}-2 h_{4} c_{0,2}{ }^{2}-h_{5} c_{0,2} c_{0,4}+h_{5} c_{0,2} c_{0,1}}{c_{0,2} \sqrt{-c_{0,1}{ }^{2}+2 c_{0,1} c_{0,4}-c_{0,4}{ }^{2}-4 c_{0,2} c_{0,3}}} \\
& d_{5}=\quad \frac{1 / 2 n_{5} c_{0,1}+h_{5} c_{0,2}-1 / 2 n_{5} c_{0,4}}{c_{0,2}} \\
& d_{6}=\quad 0 \\
& d_{7}=\quad 0 \\
& d_{8}=\frac{-h_{9} c_{0,2} c_{0,4}-c_{0,1} n_{9} c_{0,4}-c_{0,1} n_{8} c_{0,2}+h_{9} c_{0,2} c_{0,1}+1 / 2 n_{9} c_{0,1}{ }^{2}-2 h_{8} c_{0,2}{ }^{2}+c_{0,4} n_{8} c_{0,2}+1 / 2 n_{9} c_{0,4}{ }^{2}}{c_{0,2} \sqrt{-c_{0,1}{ }^{2}+2 c_{0,1} c_{0,4}-c_{0,4}{ }^{2}-4 c_{0,2} c_{0,3}}} \\
& d_{9}=\quad \frac{1 / 2 n_{9} c_{0,1}-1 / 2 n_{9} c_{0,4}+h_{9} c_{0,2}}{c_{0,2}} \\
& d_{10}=\quad 0 \\
& d_{11}=\quad 0 \\
& d_{12}=\quad 0 \\
& d_{13}=\frac{1 / 2 n_{14} c_{0,1}^{2}+1 / 2 n_{14} c_{0,4}^{2}+h_{14} c_{0,2} c_{0,1}-h_{14} c_{0,2} c_{0,4}-c_{0,1} n_{13} c_{0,2}-c_{0,1} n_{14} c_{0,4}+c_{0,4} n_{13} c_{0,2}-2 h_{13} c_{0,2}{ }^{2}}{c_{0,2} \sqrt{-c_{0,1}{ }^{2}+2 c_{0,1} c_{0,4}-c_{0,4}{ }^{2}-4 c_{0,2} c_{0,3}}} \\
& d_{14}=\quad \frac{h_{14} c_{0,2}+1 / 2 n_{14} c_{0,1}-1 / 2 n_{14} c_{0,4}}{c_{0,2}} \\
& d_{15}=\quad 0 \\
& d_{16}=\quad 0 \\
& d_{17}=\quad 0 \\
& d_{18}=\quad 0
\end{aligned}
$$


Agora, o calculo das $g_{k l}$ nos fornece:

$$
\begin{aligned}
& g_{20}=\quad 1 / 2 d_{2}-1 / 2 c_{3}+1 / 2 c_{1}+i\left(-1 / 2 c_{2}-1 / 2 d_{3}+1 / 2 d_{1}\right) \\
& g_{11}=\quad 1 / 2 c_{3}+1 / 2 c_{1}+i\left(1 / 2 d_{3}+1 / 2 d_{1}\right) \\
& g_{02}=\quad-1 / 2 d_{2}-1 / 2 c_{3}+1 / 2 c_{1}+i\left(1 / 2 c_{2}-1 / 2 d_{3}+1 / 2 d_{1}\right) \\
& g_{30}=3 / 4 d_{5}+3 / 4 c_{4}-3 / 4 d_{7}-3 / 4 c_{6}+i\left(-3 / 4 c_{5}+3 / 4 c_{7}+3 / 4 d_{4}-3 / 4 d_{6}\right) \\
& g_{21}=1 / 4 c_{6}+1 / 4 d_{5}+3 / 4 c_{4}+3 / 4 d_{7}+i\left(-1 / 4 c_{5}-3 / 4 c_{7}+3 / 4 d_{4}+1 / 4 d_{6}\right) \\
& g_{12}=1 / 4 c_{6}-1 / 4 d_{5}+3 / 4 c_{4}-3 / 4 d_{7}+i\left(1 / 4 c_{5}+3 / 4 c_{7}+3 / 4 d_{4}+1 / 4 d_{6}\right) \\
& g_{03}=-3 / 4 c_{6}-3 / 4 d_{5}+3 / 4 c_{4}+3 / 4 d_{7}+i\left(3 / 4 c_{5}-3 / 4 c_{7}+3 / 4 d_{4}-3 / 4 d_{6}\right) \\
& g_{40}=\quad-3 / 2 d_{11}-3 / 2 c_{10}+3 / 2 d_{9}+3 / 2 c_{12}+3 / 2 c_{8}+ \\
& i\left(-3 / 2 c_{9}+3 / 2 c_{11}+3 / 2 d_{8}+3 / 2 d_{12}-3 / 2 d_{10}\right) \\
& g_{31}=3 / 4 d_{9}-3 / 2 c_{12}+3 / 2 c_{8}+3 / 4 d_{11}+i\left(-3 / 4 c_{9}-3 / 4 c_{11}+3 / 2 d_{8}-3 / 2 d_{12}\right) \\
& g_{22}=\quad 3 / 2 c_{8}+1 / 2 c_{10}+3 / 2 c_{12}+i\left(3 / 2 d_{12}+1 / 2 d_{10}+3 / 2 d_{8}\right) \\
& g_{13}=3 / 2 c_{8}-3 / 2 c_{12}-3 / 4 d_{9}-3 / 4 d_{11}+i\left(-3 / 2 d_{12}+3 / 2 d_{8}+3 / 4 c_{9}+3 / 4 c_{11}\right) \\
& g_{04}=\quad-3 / 2 c_{10}+3 / 2 d_{11}-3 / 2 d_{9}+3 / 2 c_{12}+3 / 2 c_{8}+ \\
& i\left(3 / 2 c_{9}-3 / 2 c_{11}+3 / 2 d_{8}+3 / 2 d_{12}-3 / 2 d_{10}\right) \\
& g_{50}=\quad \frac{15}{4} d_{18}-\frac{15}{4} d_{16}+\frac{15}{4} c_{17}-\frac{15}{4} c_{15}+\frac{15}{4} d_{14}+\frac{15}{4} c_{13}+ \\
& i\left(-\frac{15}{4} c_{18}-\frac{15}{4} d_{15}+\frac{15}{4} d_{17}+\frac{15}{4} c_{16}-\frac{15}{4} c_{14}+\frac{15}{4} d_{13}\right) \\
& g_{41}=\quad-3 / 4 c_{15}+3 / 4 d_{16}-9 / 4 c_{17}-\frac{15}{4} d_{18}+9 / 4 d_{14}+\frac{15}{4} c_{13}+ \\
& i\left(-3 / 4 d_{15}+\frac{15}{4} d_{13}-9 / 4 d_{17}+\frac{15}{4} c_{18}-3 / 4 c_{16}-9 / 4 c_{14}\right) \\
& g_{32}=\quad 3 / 4 c_{15}+3 / 4 d_{16}+3 / 4 c_{17}+\frac{15}{4} d_{18}+3 / 4 d_{14}+\frac{15}{4} c_{13}+ \\
& i\left(-\frac{15}{4} c_{18}+3 / 4 d_{15}+3 / 4 d_{17}-3 / 4 c_{16}-3 / 4 c_{14}+\frac{15}{4} d_{13}\right) \\
& g_{23}=\quad-3 / 4 d_{16}+3 / 4 c_{15}+3 / 4 c_{17}-\frac{15}{4} d_{18}-3 / 4 d_{14}+\frac{15}{4} c_{13}+ \\
& i\left(3 / 4 d_{15}+\frac{15}{4} c_{18}+3 / 4 d_{17}+3 / 4 c_{16}+3 / 4 c_{14}+\frac{15}{4} d_{13}\right) \\
& g_{14}=\quad-3 / 4 c_{15}-3 / 4 d_{16}-9 / 4 c_{17}+\frac{15}{4} d_{18}-9 / 4 d_{14}+\frac{15}{4} c_{13}+ \\
& i\left(-\frac{15}{4} c_{18}-3 / 4 d_{15}-9 / 4 d_{17}+3 / 4 c_{16}+9 / 4 c_{14}+\frac{15}{4} d_{13}\right) \\
& g_{05}=\quad-\frac{15}{4} d_{18}+\frac{15}{4} d_{16}-\frac{15}{4} d_{14}+\frac{15}{4} c_{17}-\frac{15}{4} c_{15}+\frac{15}{4} c_{13}+ \\
& i\left(-\frac{15}{4} c_{16}+\frac{15}{4} d_{17}-\frac{15}{4} d_{15}+\frac{15}{4} d_{13}+\frac{15}{4} c_{14}+\frac{15}{4} c_{18}\right)
\end{aligned}
$$




\section{Capítulo 6}

\section{Estudo preliminar das bifurcações que ocorrem no interior de $Q \operatorname{com} \beta>0$}

Estamos estudando o sistema:

$$
\left\{\begin{array}{c}
\dot{x}=x\left(1-\lambda x-\frac{y}{\alpha x^{2}+\beta x+1}\right)=f_{1}(x, y, \alpha, \beta, \mu, \delta, \lambda) \\
\dot{y}=y\left(-\delta-\mu y+\frac{x}{\alpha x^{2}+\beta x+1}\right)=f_{2}(x, y, \alpha, \beta, \mu, \delta, \lambda)
\end{array}\right.
$$

onde os parâmetros $(\alpha, \beta, \mu, \delta, \lambda) \in \mathcal{W} \times \Delta(\mathcal{W}=\{(\alpha, \beta, \mu) ; \alpha \geq 0, \beta>-2 \sqrt{\alpha}, \mu \geq 0\}$ e $\Delta=\{(\delta, \lambda) ; \delta>0, \lambda>0\})$.

Nos capítulos anteriores, estudamos as bifurcações que ocorrem no bordo do quadrante positivo $Q$ e também estudamos as condições de não-degenerescência e transversalidade das bifurcações do tipo sela-nó e Hopf. Agora, vamos aprofundar o estudo das bifurcações de equilíbrios que ocorrem no interior de $Q$. Trataremos especificamente dos casos onde $\beta>0$. Para isso, vamos localizar candidatos para cada tipo de bifurcação de forma algébrica e geométrica.

\subsection{Estudo da região de equilíbrios}

O conjunto de parâmetros no qual vamos trabalhar é:

$$
\mathcal{W}_{1}=\left\{(\alpha, \mu, \beta) \in \mathbb{R}^{3} ;(\alpha, \beta, \mu) \in \mathcal{W}, \beta>0\right\}
$$


Para localizar os equilíbrios do sistema devemos resolver o seguinte sistema de equações:

$$
\left\{\begin{array}{c}
0=x\left(1-\lambda x-\frac{y}{\alpha x^{2}+\beta x+1}\right) \\
0=y\left(-\delta-\mu y+\frac{x}{\alpha x^{2}+\beta x+1}\right)
\end{array}\right.
$$

Como estamos fora dos eixos, podemos simplificar a expressão para:

$$
\left\{\begin{array}{c}
0=1-\lambda x-\frac{y}{\alpha x^{2}+\beta x+1} \\
0=-\delta-\mu y+\frac{x}{\alpha x^{2}+\beta x+1}
\end{array}\right.
$$

resolvendo essas equações em função de $\alpha$ e $\mu$ obtemos:

$$
\begin{gathered}
\alpha=-\frac{-\beta x-1+\lambda x^{2} \beta+\lambda x+y}{x^{2}(-1+\lambda x)}=A(x, y, \beta, \delta, \lambda) \\
\mu=-\frac{\delta y-x+\lambda x^{2}}{y^{2}}=M(x, y, \beta, \delta, \lambda)
\end{gathered}
$$

As funções $A(x, y, \beta, \delta, \lambda)$ e $M(x, y, \beta, \delta, \lambda)$ nos fornecem parâmetros $\alpha$ e $\mu$ para os quais o ponto $(x, y)$ com os parâmetros $(\alpha, \beta, \mu, \delta, \lambda)$ é um equilíbrio.

Note que, caso $x \lambda \geq 1$, temos que $M(x, y, \beta, \delta, \lambda)<0$ o que significa que o parâmetro $\mu$ relativo a esses pontos não têm interesse biológico. Assim, vamos considerar como domínio de nossas funções uma faixa que chamaremos de $\mathcal{F}$ :

\section{Definição 6.1.1.}

$$
\begin{gathered}
\mathcal{F}=\left\{(x, y, \beta, \delta, \lambda) \in \mathbb{R}^{5} ; 0<x<\frac{1}{\lambda}, y>0, \beta>0, \delta>0, \lambda>0\right\} \\
\mathcal{F}_{\delta_{0}, \lambda_{0}}=\left\{(x, y, \beta) \in \mathbb{R}^{3} ;\left(x, y, \beta, \delta_{0}, \lambda_{0}\right) \in \mathcal{F}\right\}
\end{gathered}
$$

$\operatorname{para}\left(\delta_{0}, \lambda_{0}\right) \in \Delta$

Com $\mathcal{F}$ como domínio, as funções $A(x, y, \beta, \delta, \lambda)$ e $M(x, y, \beta, \delta, \lambda)$ estão bem-definidas. Assim, estabelecemos a seguinte notação:

\section{Definição 6.1.2.}

$$
\begin{array}{rccc}
\Psi_{S}: & \mathcal{F} & \rightarrow & \mathbb{R}^{2} \\
(x, y, \beta, \delta, \lambda) & \mapsto & (A(x, y, \beta, \delta, \lambda), M(x, y, \beta, \delta, \lambda))
\end{array}
$$


Geometricamente estamos considerando os equilíbrios do sistema como um gráfico em $\mathbb{R}^{+} \times \mathbb{R}^{+} \times \mathcal{W}_{1} \times \Delta$.

Não havendo perigo de confusão, vamos nos referir às funções $A(x, y, \beta, \delta, \lambda)$ e $M(x, y, \beta, \delta, \lambda)$ como $A$ e $M$ simplesmente.

Para que os parâmetros sejam de interesse biológico, devemos ter $\alpha \geq 0$ e $\mu \geq 0$. Assim, definimos:

\section{Definição 6.1.3.}

$$
\begin{aligned}
& \mathcal{S}_{\delta_{0}, \lambda_{0}}=\left\{(x, y, \beta) \in \mathbb{R}^{3} ; 0<x<\frac{1}{\lambda_{0}}, y>0, \beta>0, A\left(x, y, \beta, \delta_{0}, \lambda_{0}\right) \geq 0, M\left(x, y, \beta, \delta_{0}, \lambda_{0}\right) \geq 0\right\} \\
& \text { para }\left(\delta_{0}, \lambda_{0}\right) \in \Delta \text { ede: } \\
& \qquad \mathcal{S}_{\beta_{0}, \delta_{0}, \lambda_{0}}=\left\{(x, y) \in \mathbb{R}^{2} ; 0<x<\frac{1}{\lambda_{0}}, y>0, A\left(x, y, \beta_{0}, \delta_{0}, \lambda_{0}\right) \geq 0, M\left(x, y, \beta_{0}, \delta_{0}, \lambda_{0}\right) \geq 0\right\} \\
& \text { para }\left(\delta_{0}, \lambda_{0}\right) \in \Delta e \beta_{0}>0 .
\end{aligned}
$$

Quando estiver claro que fixamos $(\delta, \lambda)$, vamos nos referir ao conjunto $\mathcal{S}_{\delta_{0}, \lambda_{0}}$ simplesmente como $\mathcal{S}$ para não carregar a notação. Da mesma forma, vamos nos referir ao conjunto $\mathcal{S}_{\beta_{0}, \delta_{0}, \lambda_{0}}$ simplesmente como $\mathcal{S}_{\beta_{0}}$.

O conjunto que desejamos estudar é $\mathcal{S}_{\delta, \lambda}$ para todo $(\delta, \lambda) \in \Delta$. Para isso devemos estudar o sinal de $A$ e $M$. Note que o denominador de $M$ é sempre positivo e o denominador de $A$ é sempre negativo em $\mathcal{F}$. Assim, tomando o numerador de $M$ e o numerador de $-A$ obtemos expressões suficientes para determinar o sinal de $A$ e $M$ em $\mathcal{F}$ :

$$
\begin{gathered}
P A=y+(\lambda x-1)(\beta x+1) \\
P M=-\delta y-\lambda x^{2}+x
\end{gathered}
$$

Como $P A$ e $P M$ são contínuas e queremos que eles sejam positivos, vamos começar verificando onde que eles são iguais a zero. Fixado $(\delta, \lambda) \in \Delta$ e $\beta>0, P A=0$ e $P M=0$ são parábolas. De fato, podemos isolar $y$ de forma a exprimir as curvas como gráficos:

$$
\begin{gathered}
y A=-(\lambda x-1)(\beta x+1) \\
y M=-\frac{x(\lambda x-1)}{\delta}
\end{gathered}
$$




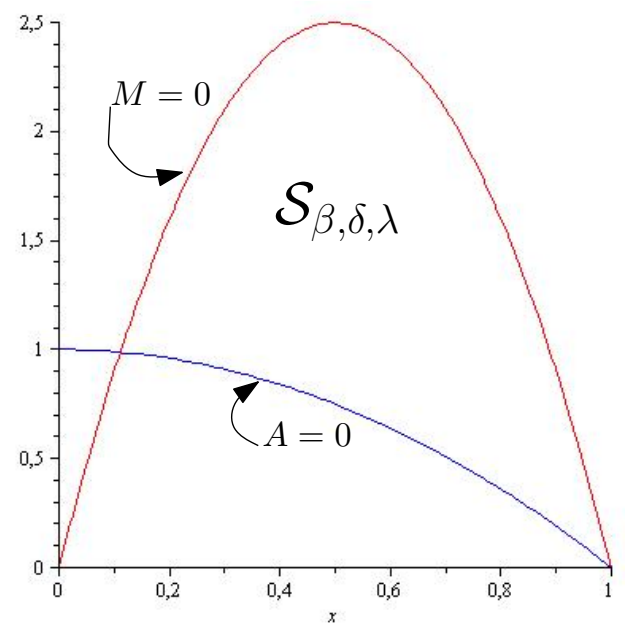

Figura 6.1: Em azul a curva $P A=0$ e em vermelho a curva $P M=0$. Os equilíbrios do sistema se encontram abaixo da parábola vermelha e acima da azul. Este conjunto é igual a $\mathcal{S}_{\delta, \lambda \beta} \operatorname{com} \beta=1$, $\delta=0.1$ e $\lambda=1$.

No caso de $P M=0$ temos uma parábola cujos zeros se encontram em $x=0$ e $x=\frac{1}{\lambda}$. Já no caso de $P A=0$ obtemos uma parábola cujos zeros estão em $x=\frac{1}{\lambda}$ e $-\frac{1}{\beta}<0$. Além disso, a curva $P A=0$ passa pelo ponto $(0,1)$. Analisando os sinais de $P A$ e $P M$ nota-se que, fixado $\beta$, temos $A>0$ caso $(x, y)$ esteja acima da parábola $P A=0$ e $M>0$ caso $(x, y)$ esteja abaixo de parábola $P M=0$. Ver figura 6.1 .

Assim, $\mathcal{S}_{\beta}$ é o conjunto de pontos abaixo da parábola $P M=0$ e acima da parábola $P A=0$. Vamos obter algumas informações sobre este conjunto:

Lema 6.1.4. Para $\left(\delta_{0}, \lambda_{0}\right) \in \Delta$ fixado temos que:

- $\mathcal{S}$ é diferente de vazio se, e somente se, $\delta_{0} \lambda_{0}<1$;

- $\mathcal{S}_{\beta_{0}}$ é diferente de vazio se, e somente se, $\delta_{0}\left(\beta_{0}+\lambda_{0}\right)<1$;

Demonstração. Para que $\mathcal{S}_{\beta}$ seja diferente de vazio, a curva $P M=0$ deve ficar acima da curva $P A=0$. Como a curva $P A=0$ passa pelo ponto $(0,1)$ e a curva $P M=0$ passa pela origem, $\mathcal{S}_{\beta}$ é não vazio caso essas curvas se cruzem com $0<x<\frac{1}{\lambda}$. Tomando a equação 
$y A=y M$ e resolvendo em função de $x$ obtemos.

$$
\begin{gathered}
x=\frac{1}{\lambda}, \\
x=\frac{\delta}{1-\delta \beta}
\end{gathered}
$$

Assim, para que estejamos tratando de um caso cujo conjunto de equilíbrios seja diferente de vazio, devemos ter $0<\frac{\delta}{1-\delta \beta}<\frac{1}{\lambda}$ o que significa que:

$$
\begin{gathered}
\beta \delta<1, \\
\delta(\beta+\lambda)<1
\end{gathered}
$$

Quando $\beta=0$, devemos ter apenas $\delta \lambda<1$. Assim, caso fixemos $(\delta, \lambda) \in \Delta$ tal que $\delta \lambda<1$ então teremos equilíbrios para o sistema para $\beta<\frac{1-\delta \lambda}{\delta}$.

Corolário 6.1.5. Fixado $\left(\delta_{0}, \lambda_{0}\right) \in \Delta$ :

- fixado $\beta_{0}>0$, o conjunto $\mathcal{S}_{\beta_{0}}$ é conexo.

- o conjunto $\mathcal{S}$ é conexo;

Demonstração. Como $\mathcal{S}_{\beta_{0}}$ é o conjunto entre duas parábolas, ele é obviamente conexo. Agora, para cada $\beta<\frac{1-\delta \lambda}{\delta}, \mathcal{S}_{\beta}$ é diferente de vazio e conexo. Como $\mathcal{S}=\bigcup_{\beta<\frac{1-\delta \lambda}{\delta}}\left(\mathcal{S}_{\beta} \times \beta\right)$ e a variação da fronteira é contínua, pois $A$ e $M$ são analíticas em $\mathcal{F}, \mathcal{S}$ é conexo.

O próximo passo é estudar se um equilíbrio é ou não é hiperbólico. Para que um equilíbrio seja não hiperbólico, uma das seguintes condições devem ser satisfeitas:

- o determinante do Jacobiano do sistema em relação as variáveis dinâmicas $(x, y)$, calculado no equilíbrio deve ser nulo

- o determinante deve ser positivo e o traço do Jacobiano do sistema em relação as variáveis dinâmicas $(x, y)$, calculado no equilíbrio deve ser nulo

Nas duas próximas secções estudaremos esses casos. A partir de agora, vamos nos referir ao Jacobiano do sistema em relação as variáveis dinâmicas $(x, y)$, calculado no equilíbrio simplesmente como Jacobiano (se houver dúvida quanto ao equilíbrio, o explicitaremos). 


\subsection{Estudo dos equilíbrios não hiperbólicos nos quais o determinante é igual a 0}

Começamos a nossa análise dos equilíbrios não hiperbólicos pelos equilíbrios cujo linearizado tenha determinante nulo. Para isso basta verificar em quais pontos o determinante do jacobiano em relação a $x$ e $y$ da aplicação $\Psi_{S}$ se anula.

\section{Definição 6.2.1.}

$$
\begin{array}{cccc}
W: & \mathcal{F} & \rightarrow & \mathbb{R} \\
(x, y, \beta, \delta, \lambda) & \mapsto & \frac{\partial(A, M)}{\partial(x, y)}(x, y, \beta, \delta, \lambda)
\end{array}
$$

Assim, temos que:

$$
W=\frac{1}{x^{3}(-1+\lambda x)^{2} y^{3}}\left(a_{w} y^{2}+b_{w} y+c_{w}\right)
$$

onde:

$$
\begin{gathered}
a_{w}=3 \lambda x \delta-2 \delta \\
b_{w}=(-1+\lambda x)\left(\lambda x^{2} \beta \delta+4 \lambda x^{2}+2 \lambda x \delta-3 x-\delta \beta x-2 \delta\right) \\
c_{w}=2 x(-1+\lambda x)^{3}(\beta x+2)
\end{gathered}
$$

Notemos primeiro que o denominador de $W$ :

$$
x^{3}(-1+\lambda x)^{2} y^{3}
$$

é não nulo e sempre positivo em $\mathcal{F}$. Assim, $W$ está bem definido para todo ponto em $\mathcal{F}$ e, consequentemente, para todo ponto em $\mathcal{S}_{\delta, \lambda}$, qualquer $(\delta, \lambda) \in \Delta$.

Assim, estamos interessados em estudar a superfície $W=0$ restrita a $\mathcal{S}_{\delta, \lambda}$. Chamaremos essa superfície de $\mathcal{W S}$ :

Definição 6.2.2. Para $(\delta, \lambda) \in \Delta$ fixo:

$$
\mathcal{W} \mathcal{S}_{\delta, \lambda}=\left\{p \in \mathcal{S}_{\delta, \lambda} ; W(p)=0\right\}
$$

Quando estiver claro que fixamos $(\delta, \lambda) \in \Delta$, vamos nos referir a este conjunto simplesmente como $\mathcal{W S}$. 


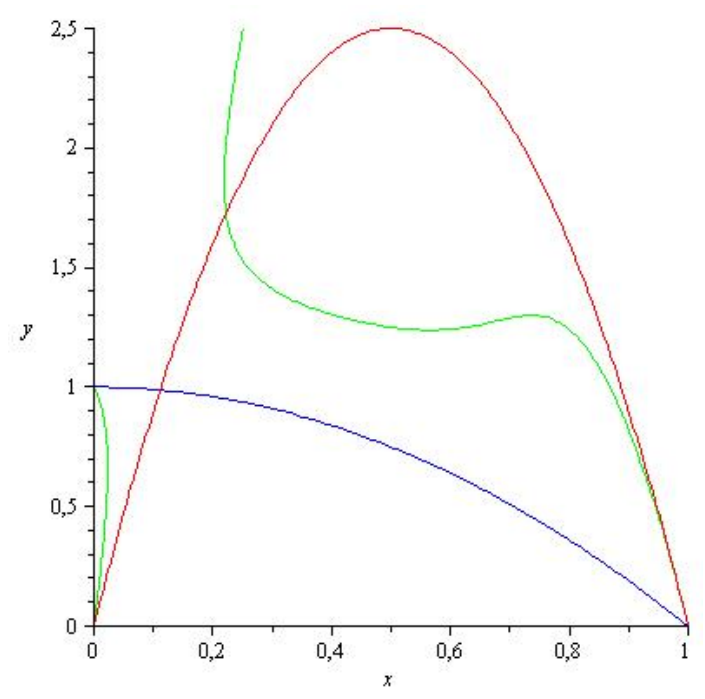

Figura 6.2: Em azul a curva $P A=0$, em vermelho a curva $P M=0$ e em verde a curva $W=0$. Todos os pontos em $\mathcal{S}$ em verde são equilíbrios não-hiperbólicos para $\alpha=A$ e $\mu=M$. Para obter a figura fixamos $\delta=0.1, \lambda=1$ e $\beta=1$.

Vamos estudar o conjunto $\mathcal{W S}$ : quantas componentes conexas existem e se é uma superfície regular. Esta superfície representa as dobras da aplicação $\Psi_{S}$. No entanto, o mais importante é que, do ponto de vista dinâmico, ela será igual a superfície onde ocorrem bifurcações do tipo $S N_{1}$ e suas degenerescências $\left(S N_{2}, B T\right.$ etc). Assim, a usaremos para obter informações sobre a superfície de dobra geral, definida no capítulo 4 .

\subsubsection{Estratégia}

Queremos estudar a superfície $W=0$. Inicialmente ela deve ser, genericamente, uma hipersuperfície de $\mathbb{R}^{5}$. No entanto, vamos fixar $(\delta, \lambda) \in \Delta$ e $\beta>0$. Dessa forma, devemos ter, genericamente, uma curva em $\mathbb{R}^{2}$.

Começaremos nossa análise achando uma parametrização para as curvas $W=0$. Isso será facilmente obtido pois $W$ é uma expressão quadrática em y e portanto temos dois ramos de 
solução. Com esses ramos, provaremos que só podem existir duas curvas conexas na faixa $\mathcal{F}$ e que apenas uma delas intersecta $\mathcal{S}$. Em seguida, analisaremos quando que $A \geq 0$.

Por fim, utilizaremos a análise realizada até então para obter alguns resultados acerca da superfície de dobra geral definida no capítulo 4.

\subsubsection{Parametrização dos ramos}

Notemos que $W$ é quadrática em $y$ e portanto, quando o coeficiente $a_{w}$ for não nulo, podemos parametrizar a superfície como dois gráficos de $x, \beta, \delta$ e $\lambda$ em $y$. Assim, temos dois ramos de solução:

$$
\begin{aligned}
& y_{1}=\frac{-b_{w}-\sqrt{b_{w}^{2}-4 a_{w} c_{w}}}{2 a_{w}} \\
& y_{2}=\frac{-b_{w}+\sqrt{b_{w}^{2}-4 a_{w} c_{w}}}{2 a_{w}}
\end{aligned}
$$

onde a união de $\left(x, y_{1}, \beta, \delta, \lambda\right)$ e $\left(x, y_{2}, \beta, \delta, \lambda\right)$ é igual a superfície $W=0$.

No caso em que o coeficiente $a_{w}$ se anule, então $b_{w} \neq 0$. De fato, $a_{w}=0$ quando $x=\frac{2}{3 \lambda} \mathrm{e}$ calculando $b_{w}$ em $x=\frac{2}{3 \lambda}$ obtemos $\frac{2}{27} \frac{\delta \beta+1+3 \lambda \delta}{\lambda}$ que é sempre não nulo. Neste ponto temos uma única solução em $y$. Esta solução já faz parte de um dos ramos anteriores:

Lema 6.2.5. Quando $x=\frac{2}{3 \lambda}$, o ramo $\left(x, y_{1}, \beta, \delta, \lambda\right)$ não está bem-definido mas o ramo $\left(x, y_{2}, \beta, \delta, \lambda\right)$ está. Além disso, fixado $(\delta, \lambda) \in \Delta e \beta>0$, a curva $\left(x, y_{2}, \beta, \delta, \lambda\right)$ é regular quando $x=\frac{2}{3 \lambda}$.

Demonstração. Notemos que o numerador de $y_{1}$ no ponto $x=\frac{2}{3 \lambda}$ é diferente de zero enquanto o seu numerador é igual a zero. Assim, $y_{1}$ não está definido neste ponto. Já o numerador de $y_{2}$ calculado no ponto $x=\frac{2}{3 \lambda}$ é igual a zero e portanto, a priori, não podemos afirmar nada. Usando l'Hospital podemos provar que o limite existe. Além disso, fixando $(\delta, \lambda) \in \Delta$ e $\beta>0$, o numerador de $y_{2}$ é analítico em relação a $x$, e pode localmente ser escrito como uma função analítica multiplicada pelo denominador. Assim a curva $\left(x, y_{2}\right)$ é analítica em relação a $x$ neste ponto.

Assim, temos uma parametrização para $W=0$. Devemos nos preocupar com a validade destas parametrizações: 
Lema 6.2.6. O ramo $\left(x, y_{1}, \beta, \delta, \lambda\right)$ não é admíssivel caso $\frac{2}{3 \lambda}<x<\frac{1}{\lambda}$.

Demonstração. Afirmo que $y_{1}<0$ caso $x>\frac{2}{3 \lambda}$ e portanto o ramo é não admissível. De fato, caso $x>\frac{2}{3 \lambda}$, então, claramente, $a_{w}>0$. Além disso, $c_{w}<0$ qualquer que seja $0<x \lambda<1$. Assim, $a_{w} c_{w}<0$ e portanto: $b_{w}^{2}-4 a_{w} c_{w}>b_{w}^{2}$. Dessa forma:

$$
-b_{w}-\sqrt{b_{w}^{2}-4 a_{w} c_{w}>b_{w}^{2}}<0
$$

e, portanto, $y_{1}<0$.

Assim, só devemos nos preocupar com $\left(x, y_{1}, \beta, \delta, \lambda\right)$ caso $x<\frac{2}{3 \lambda}$. Já para o outro ramo é diferente:

Lema 6.2.7. $y_{2}$ é sempre positivo quando $\frac{2}{3 \lambda}<x<\frac{1}{\lambda}$.

Demonstração. Pela mesma análise anterior, temos $b_{w}^{2}-4 a_{w} c_{w}>b_{w}^{2}$. Logo:

$$
-b_{w}+\sqrt{b_{w}^{2}-4 a_{w} c_{w}}>0
$$

e, portanto, $y_{2}>0$.

Além disso, é fácil ver que ambas as curvas se aproximam de $y=0$ quando $x$ se aproxima de $\frac{1}{\lambda}$.

Ainda precisamos estudar os ramos quando $x<\frac{2}{3 \lambda}$. O primeiro resultado que podemos provar nos mostrará que esses ramos só tem uma possibilidade de não serem válidos:

Lema 6.2.8. Fixado $(\delta, \lambda) \in \Delta$, tomando $0<x<\frac{2}{3 \lambda}$, então, caso $y_{1}$ e $y_{2}$ sejam reais, eles serão positivos.

Demonstração. Comecemos notando que $a_{w}<0$ neste intervalo. Estamos assumindo que $b_{w}^{2}-4 a_{w} c_{w} \geq 0$. No entanto, como $a_{w}>0$ e $c_{w}>0, b_{w}^{2}-4 a_{w} c_{w}<b_{w}^{2}$ e, portanto, para identificar o sinal de $y_{1}$ e $y_{2}$, basta verificar o sinal de $\frac{-b_{w}}{a_{w}}$. Para que ele seja positivo, devemos ter $b_{w}>0$ neste intervalo. Para isso, vamos achar as raizes de $b_{w}$ em relação a x:

$$
\begin{array}{ll}
x 0= & \frac{1}{\lambda} \\
x 1= & 1 / 2 \frac{3-2 \lambda \delta+\delta \beta+\sqrt{9+20 \lambda \delta+6 \delta \beta+4 \lambda^{2} \delta^{2}+4 \lambda \beta \delta^{2}+\delta^{2} \beta^{2}}}{\lambda(4+\delta \beta)} \\
x 2= & -1 / 2 \frac{-3+2 \lambda \delta-\delta \beta+\sqrt{9+20 \lambda \delta+6 \delta \beta+4 \lambda^{2} \delta^{2}+4 \lambda \beta \delta^{2}+\delta^{2} \beta^{2}}}{\lambda(4+\delta \beta)}
\end{array}
$$


Não é difícil ver que $x 0$ e $x 2$ não pertencem a faixa $\mathcal{F}$ e que $x 1$ é sempre maior do que $\frac{2}{3 \lambda}$. Isso implica que $b_{w}$ conserva sempre o mesmo sinal quando $0<x<\frac{2}{3 \lambda}$. Por fim, avaliando $b_{w}$ em qualquer ponto desse intervalo, obtemos um valor positivo e assim, $b_{w}$ é sempre positivo neste intervalo. Isso encerra a demonstração.

Assim, a única forma de $y_{1}$ e $y_{2}$ não serem válidos para $0<x<\frac{2}{3 \lambda}$ é eles se tornarem complexos. Assim devemos nos preocupar com o discriminante de $W$ em $y$ :

\section{Definição 6.2.9.}

$$
\begin{gathered}
\text { disc }=b_{w}^{2}-4 a_{w} c_{w}=(1-\lambda x)^{2}\left(\left(16 \lambda^{2}+\lambda^{2} \beta^{2} \delta^{2}-16 \lambda^{2} \beta \delta\right) x^{4}+\right. \\
\left(-32 \lambda^{2} \delta+4 \lambda^{2} \beta \delta^{2}-2 \lambda \beta^{2} \delta^{2}-24 \lambda+26 \lambda \beta \delta\right) x^{3} \\
+\left(52 \lambda \delta-10 \delta \beta+9+4 \lambda^{2} \delta^{2}-8 \lambda \beta \delta^{2}+\delta^{2} \beta^{2}\right) x^{2} \\
\left.+\left(-20 \delta+4 \delta^{2} \beta-8 \lambda \delta^{2}\right) x+4 \delta^{2}\right)
\end{gathered}
$$

O fator da função disc que pode se anular em $\mathcal{F}$, é quártico na variável $x$. Dessa forma, fixando $\delta, \lambda$ e $\beta$ deveríamos trabalhar com a hipótese de existirem até três curvas desconexas na faixa, sendo uma delas uma ilha. No entanto, vamos mostrar que não ocorre nenhuma ilha e que a configuração que nos interessa sempre possui duas curvas conexas e um espaço entre elas, caracterizado pelo discriminante negativo e, consequentemente, por ramos complexos.

\subsubsection{Estudo da função disc}

Nesta secção, temos como intuito verificar quantas curvas conexas de $W=0$ podem ocorrer em $\mathcal{F}$ para $(\delta, \lambda) \in \Delta$ e $\beta>0$ fixos. Pelos últimos resultados, isto é equivalente a procurar o número de zeros da função disc para $0<x<\frac{1}{\lambda}, \beta>0$ e $(\delta, \lambda) \in \Delta$.

Pela continuidade de disc, ao mudarmos os parâmetros $(\delta, \lambda, \beta)$ de forma contínua, o surgimento ou o desaparecimento de uma raiz não pode ser "brusco", ou seja, deve ocorrer uma bifurcação nas raízes. Assim, vamos estudar as bifurcações de codimensão um das raízes de disc. Vamos considerar $x$ como a variável dinâmica e $(\beta, \lambda, \delta)$ os parâmetros. 
Vamos começar mostrando que a função disc não recebe raízes dos extremo de seu domínio:

Lema 6.2.11. Para todos os valores dos parâmetros $(\beta, \delta, \lambda)$ :

- $x=0$ não é raiz de disc;

- $x=\frac{1}{\lambda}$ é raiz dupla de disc.

Demonstração. Comecemos por $x=0$ :

$$
\operatorname{disc}(0)=4 \delta^{2} \neq 0
$$

Já para $x=\frac{1}{\lambda}$ devemos fazer a seguinte consideração:

$$
\frac{\operatorname{disc}}{(1-\lambda x)^{2}}\left(\frac{1}{\lambda}\right)=\frac{1}{\lambda^{2}} \neq 0
$$

Assim, este extremo permanece como um zero de multiplicidade dois para todos os valores dos demais parâmetros.

Desta forma, a única forma de aparecerem raízes com a variação dos parâmetros é aparecerem raízes de multiplicidade dois ou maiores no interior do domínio da função disc. Para nos auxiliar neste estudo, vamos começar fazendo uma ligação entre as raízes dupla da função disc e o gradiente da função $W$ :

Lema 6.2.12. Fixado $(\delta, \lambda) \in \Delta e \beta>0$, então:

- $W_{y}(p) \neq 0$ se, e somente se $\operatorname{disc}\left(x_{p}\right) \neq 0$

- $\operatorname{se} \operatorname{disc}\left(x_{p}\right)=0$, então $W_{x}(p) \neq 0$ se, e somente se, $\operatorname{disc}_{x}\left(x_{p}\right) \neq 0$.

ou seja, caso o gradiente de $W$ seja diferente de zero em $\left(x_{0}, \beta_{0}, \delta_{0}, \lambda_{0}\right)$ então $x_{0}$ ou não é raiz ou é raiz simples de disc.

Demonstração. Para começar, notemos que, quando estamos sobre $W=0$, tomar o gradiente de $W$ ou o gradiente do numerador de $W$ é indiferente. Chamaremos de $w n$ o numerador de $W$. Comecemos notando que, caso $\operatorname{disc}\left(x_{p}\right)=0$, então, $y_{1}=y_{2}$ e, portanto, $y=-\frac{b_{w}}{2 a_{w}}$. Substituindo em $w n_{y}$, temos que $w n_{y}\left(x_{p},-\frac{b_{w}}{2 a_{w}}\right)=0$. Assim, pela contra positiva, caso 
$w n_{y}(p) \neq 0$, então $\operatorname{disc}\left(x_{p}\right) \neq 0$. Além disso, é fácil ver que, caso $w n_{y}=0$, então $y=-\frac{b_{w}}{2 a_{w}}$ $\mathrm{e}$, portanto, $\operatorname{disc}\left(x_{p}\right)=0$.

Agora, suponha que $\operatorname{disc}\left(x_{p}\right)=0$ e, portanto $w n_{y}(p)=0$. Afirmo que se $w n\left(x_{p}\right)$ for não nulo, então $x_{p}$ não pode ser zero de multiplicidade maior do que um da função disc. De fato, resolvendo a equação $\operatorname{disc}\left(x_{p}\right)=0$ em função de $\delta$, obtemos:

$$
\begin{aligned}
d 1 & =\frac{\left(8 \lambda x-5+2 \sqrt{12 \lambda^{2} x^{2}-14 \lambda x+4}\right) x}{(-1+\lambda x)(\beta x+2)} \\
d 2 & =\frac{\left(8 \lambda x-5-2 \sqrt{12 \lambda^{2} x^{2}-14 \lambda x+4}\right) x}{(-1+\lambda x)(\beta x+2)}
\end{aligned}
$$

Que só são válidos caso $0<x \lambda \leq \frac{1}{2}$. Agora, para que $x_{p}$ seja um zero de multiplicidade maior do que um de disc, devemos ter $\operatorname{disc}_{x}\left(x_{p}\right)=0$. Vamos mostrar que isso não é possível caso $w n_{x}(p) \neq 0$. Para isso, lembremos que $y_{p}$ deve ser igual a $-\frac{b_{w}}{2 a_{w}}$ e que $\delta=d_{1}$ ou $d_{2}$ com $0<x \lambda \leq \frac{1}{2}$. Assim, dividindo $\operatorname{disc}_{x}\left(x, \beta, d_{1}, \lambda\right)$ por $w n_{x}\left(x,-\frac{b_{w}}{2 a_{w}}, \beta, d_{1}, \lambda\right)$ obtemos:

$$
\frac{\operatorname{disc}_{x}\left(x, \beta, d_{1}, \lambda\right)}{w n_{x}\left(x,-\frac{b_{w}}{2 a_{w}}, \beta, d_{1}, \lambda\right)}=-4 \frac{(8 \lambda x-5+2 \sqrt{2} \sqrt{(2 \lambda x-1)(3 \lambda x-2)}) x(3 \lambda x-2)}{(-1+\lambda x)(\beta x+2)}
$$

Note que o denominador e o numerador não se anulam em para $0<x \lambda \leq \frac{1}{2}$. Assim, temos:

$$
\operatorname{disc}_{x}\left(x, \beta, d_{1}, \lambda\right)=p_{1}(x, \beta, \lambda) w n_{x}\left(x,-\frac{b_{w}}{2 a_{w}}, \beta, d_{1}, \lambda\right)
$$

onde $p_{1} \neq 0 . \quad \operatorname{Logo}_{\operatorname{lisc}} \operatorname{disc}_{x}\left(x, \beta, d_{1}, \lambda\right)=0$ se, e somente se, $w n_{x}\left(x,-\frac{b_{w}}{2 a_{w}}, \beta, d_{1}, \lambda\right)=0$. Analogamente, substituindo $\delta$ por $d_{2}$, obtemos:

$$
\frac{\operatorname{disc}_{x}\left(x, \beta, d_{2}, \lambda\right)}{w n_{x}\left(x,-\frac{b_{w}}{2 a_{w}}, \beta, d_{2}, \lambda\right)}=4 \frac{(-8 \lambda x+5+2 \sqrt{2} \sqrt{(2 \lambda x-1)(3 \lambda x-2)}) x(3 \lambda x-2)}{(-1+\lambda x)(\beta x+2)}
$$

Assim, $\operatorname{disc}_{x}\left(x, \beta, d_{2}, \lambda\right)=0$ se, e somente se $w n_{x}\left(x,-\frac{b_{w}}{2 a_{w}}, \beta, d_{2}, \lambda\right)=0$. Logo, caso $W_{x}(p) \neq$ $0, \operatorname{disc}_{x}\left(x_{p}\right) \neq 0$ e $x_{p}$ é uma raiz simples de disc.

Desta forma, procurar os zeros duplos de disc é o mesmo que procurar os pontos onde o gradiente de $W$ se anula:

Lema 6.2.13. O conjunto de pontos onde $W, W_{x}$ e $W_{y}$ se anulam simultaneamente é uma superfície: $\left(x, \frac{-b_{w}}{2 a_{w}}(x, \lambda), \beta_{2}(x, \lambda), \delta_{2}(x, \lambda), \lambda\right)$, onde $0<x \lambda<\frac{7-\sqrt{17}}{8}$. Quando projetamos ela nos parâmetros $(\beta, \delta, \lambda)$, ela permanece uma superfície. 
Demonstração. De $W=0$, obtemos $y=-\frac{b_{w}}{2 a_{w}}$. Além disso, como $W_{y}=0$, então disc $=0$. Assim, vamos resolver a equação disc $=0$ em função de $\delta$ :

$$
\begin{aligned}
& d 1=\frac{\left(8 \lambda x-5+2 \sqrt{12 \lambda^{2} x^{2}-14 \lambda x+4}\right) x}{(-1+\lambda x)(\beta x+2)} \\
& d 2=\frac{\left(8 \lambda x-5-2 \sqrt{12 \lambda^{2} x^{2}-14 \lambda x+4}\right) x}{(-1+\lambda x)(\beta x+2)}
\end{aligned}
$$

Ambos são reais e positivos caso $\lambda x<\frac{1}{2}$. Agora, substituindo $d_{1}$ em $W_{x}$ e resolvendo a equação $W_{x}=0$ em $\beta$ obtemos uma equação para $\beta$ que depende apenas de $x$ e $\lambda$ (o fator que multiplica $\beta$ é sempre não nulo para $0<x \lambda \leq \frac{1}{2}$ ). Tal $\beta$ é sempre negativa quando $0<x \lambda<\frac{1}{2}$ e portanto podemos descarta-la. Já quando fazemos a mesma conta com $d_{2}$ obtemos uma expressão para $\beta$ que chamaremos de $\beta_{2}$ :

$$
\beta_{2}=2 \frac{-24 \lambda^{3} x^{3}+62 \lambda^{2} x^{2}-42 \lambda x+8\left(+8 \lambda^{2} x^{2}-16 \lambda x+5\right) \sqrt{2} \sqrt{(2 \lambda x-1)(-2+3 \lambda x)}}{\lambda x^{2}(-10 \lambda x+3 \sqrt{2} \sqrt{(2 \lambda x-1)(-2+3 \lambda x)}+6)}
$$

essa expressão é positiva quando $x \lambda<\frac{7-\sqrt{17}}{8}$. Assim, vamos assumir $x \lambda<\frac{7-\sqrt{17}}{8}, \beta=\beta_{2} \mathrm{e}$ $\delta=\delta_{2}$ onde $\delta_{2}$ é $d_{2} \operatorname{com} \beta=\beta_{2}$.

Claramente o conjunto $\left(x, \frac{-b_{w}}{2 a_{w}}(x, \lambda), \beta_{2}(x, \lambda), \delta_{2}(x, \lambda), \lambda\right)$ é uma superfície de dimensão dois (a diferencial desta aplicação tem, claramente, posto dois). Mas, resta provar que apenas $\left(\beta_{2}(x, \lambda), \delta_{2}(x, \lambda), \lambda\right)$ também é uma aplicação com posto dois e portanto uma superfície em $\mathcal{W}_{\beta} \times \Delta$. Para isso, basta verificar que $\left(\beta_{2}\right)_{x}$ e $\left(\delta_{2}\right)_{x}$ não se anulam simultaneamente. De fato $\left(\beta_{2}\right)_{x}$ é uma função não nula para $0<x \lambda<\frac{7-\sqrt{17}}{8}$. Basta tomar a derivada e mostrar que o numerador é diferente de zero. O numerador é do tipo:

$$
p(x, \lambda)+q(x, \lambda) \sqrt{h(x, \lambda}
$$

Uma condição necessária para esta expressão se anular é $p^{2}-q^{2} h=0$, ou seja:

$$
\lambda x\left(1391 \lambda^{5} x^{5}-4452 \lambda^{4} x^{4}+5682 \lambda^{3} x^{3}-3612 \lambda^{2} x^{2}+1143 \lambda x-144\right)(-3+4 \lambda x)^{2}=0
$$

o que não ocorre para $x \lambda<\frac{7-\sqrt{17}}{8}$.

Por fim, substituindo esses valores nas derivadas de disc com respeito a $x$ obtemos:

$$
\begin{gathered}
\operatorname{disc}_{x}\left(x,-\frac{b_{w}}{2 a_{w}}, \beta_{2}, \delta_{2}, \lambda\right)=0 \\
\operatorname{disc}_{x, x}\left(x,-\frac{b_{w}}{2 a_{w}}, \beta_{2}, \delta_{2}, \lambda\right)>0
\end{gathered}
$$


Tais afirmações são válidas para $0<x<\frac{7-\sqrt{17}}{8}$.

Desta forma, chegamos a conclusão de que a superfície no espaço de parâmetros onde ocorre zeros duplos da função discriminante é conexa. Agora, vamos obter informações sobre o número de raízes da função disc quando $(\delta, \lambda) \in \Delta$ estiver fixo.

Lema 6.2.14. Fixado $\lambda>0$, a equação $\delta_{2}(x)$ é inversível. Tal inversão é válida para qualquer $\delta>0$.

Demonstração. Comecemos mostrando que essa função é injetora para $0<x \lambda<\frac{1}{2}$ : O denominador da derivada de $\delta_{2}(x)$ não se anula, uma vez que o denominador de $\delta_{2}(x)$ não se anula. Tomando o numerador, temos uma expressão do tipo:

$$
p(x, \lambda)+q(x, \lambda) \sqrt{h(x, \lambda)}
$$

Assim, para que o numerador seja igual a zero, devemos ter $p^{2}-q^{2} h=0$, ou seja:

$$
-\lambda x\left(1391 \lambda^{5} x^{5}-4452 \lambda^{4} x^{4}+5682 \lambda^{3} x^{3}-3612 \lambda^{2} x^{2}+1143 \lambda x-144\right)(4 \lambda x-3)^{4}=0
$$

o que não ocorre para $0<x \lambda<\frac{1}{2}$. Assim sendo, essa função é injetora neste domínio. Além disso, temos que:

$$
\begin{gathered}
\delta_{2}(0)=0 \\
\text { denominador }\left(\delta_{2}\left(\frac{1}{2 \lambda}\right)\right)=0 \\
\text { numerador }\left(\delta_{2}\left(\frac{1}{2 \lambda}\right)\right)=-\frac{1}{4 \lambda}
\end{gathered}
$$

Desta forma, para todo $\delta_{0}>0$, existe $0<x_{0}<\frac{1}{2}$ tal que $\delta_{2}\left(x_{0}\right)=\delta_{0}$.

Assim sendo, fixado $(\delta, \lambda) \in \Delta$, existe um único ponto $\left(x_{0}, \beta_{0}\right)$ onde a função disc possui um zero duplo. Isso implica o seguinte corolário:

Corolário 6.2.15. A superfície de raízes duplas da função disc divide o espaço de parâmetros $(\beta, \delta, \lambda)$ em duas componentes conexas.

Demonstração. A superfície de raízes duplas da função disc é $\left(\beta_{2}(x, \lambda), \delta_{2}(x, \lambda), \lambda\right)$ com $0<$ $x \lambda<\frac{7-\sqrt{17}}{8}$. Pelo lema anterior, fixado $\left(\lambda_{0}, \delta_{0}\right)$ existe um único $x_{0}$ tal que $\delta_{2}\left(x_{0}, \lambda_{0}\right)=\delta_{0}$. Assim sendo, fixado $\left(\delta_{0}, \lambda_{0}\right) \in \Delta$ existe um único $\beta_{0}>0$ tal que $\left(\beta_{0}, \delta_{0}, \lambda_{0}\right) \in\left(\beta_{2}(x, \lambda), \delta_{2}(x, \lambda), \lambda\right)$. Portanto o espaço de parâmetros é dividido entre aqueles acima e aqueles abaixo da superfície de raízes duplas. 

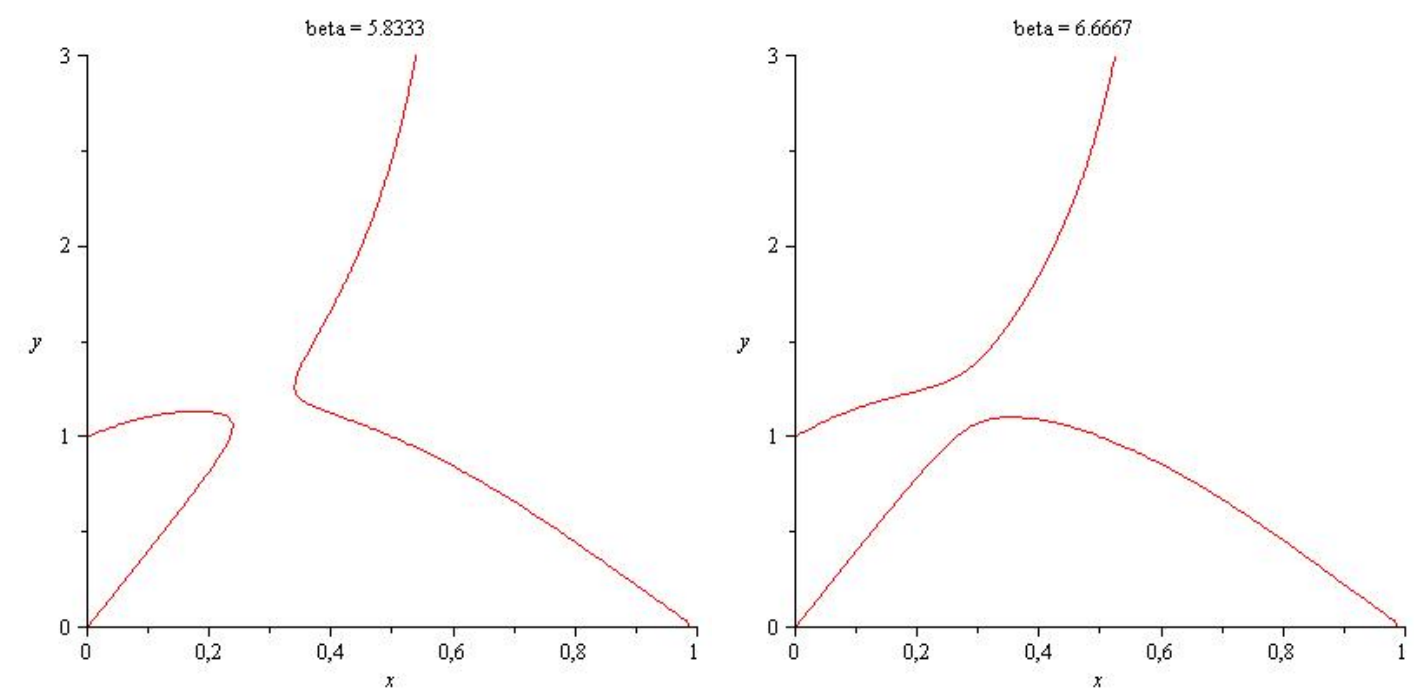

Figura 6.3: Em vermelho a curva $W=0$. Para obter a figura fixamos $\delta=0.5$ e $\lambda=1$.

Estamos prontos para a seguinte proposição:

Proposição 6.2.16. Qualquer que seja $(\delta, \lambda) \in \Delta$ e $\beta$ fixos, a função disc possui: 0 ou 1 ou 2 zeros.

Demonstração. Como não aparecem raízes nos extremos do domínio da função disc, novas raízes só ocorrem caso exista um zero duplo desta função. No espaço de parâmetros, a superfície de zeros duplos da função disc é conexa divide $(\beta, \delta, \lambda)$ em duas componentes conexas. Em cada uma delas o número de equilíbrios é fixo. Assim, temos que:

- a função disc possui: 0 ou 1 ou 2 zeros ou

- a função disc possui: 1 ou 2 ou 3 zeros ou

- a função disc possui: 2 ou 3 ou 4 zeros

Para encerrar a demonstração, basta a análise de um único caso. Podemos faze-la, inclusive, de modo gráfico, como na figura 6.3: Na esquerda, o discriminante se anula duas vezes. Já na direita, o discriminante não se anula. Isso conclui a proposição. 


\subsubsection{Estudo preliminar da intersecção de $W=0 \operatorname{com} \mathcal{S}$}

Comecemos limitando ainda mais as opções da secção anterior:

Lema 6.2.18. Para $(\delta, \lambda) \in \Delta$ fixo, para todo $\beta>0$ tal que $\mathcal{S}_{\beta}$ é não vazio, a função disc possui dois zeros.

Demonstração. Comecemos lembrando que uma condição necessária e suficiente para que, fixado um $\beta>0, \mathcal{S}_{\beta}$ seja diferente de vazio é:

$$
\delta \lambda+\delta \beta-1<0
$$

Assim, ao substituir $\delta$ por $\delta_{2}$ e $\beta$ por $\beta_{2}$ em $\delta \lambda+\delta \beta-1$ e fazer a mudança de variáveis $x \lambda=z$ obtemos:

$$
\begin{gathered}
8\left(z \left(1944-16563 z-27152 z^{5}-6582 \sqrt{2} \sqrt{(2 z-1)(-2+3 z)} z\right.\right. \\
-95407 z^{3}-18700 z^{3} \sqrt{2} \sqrt{(2 z-1)(-2+3 z)}+7840 z^{4} \sqrt{2} \sqrt{(2 z-1)(-2+3 z)} \\
\left.\left.+16668 z^{2} \sqrt{2} \sqrt{(2 z-1)(-2+3 z)}+56298 z^{2}+80600 z^{4}+972 \sqrt{2} \sqrt{(2 z-1)(-2+3 z)}\right)\right) \\
\left(-24 z^{2}+8 \sqrt{2} \sqrt{(2 z-1)(-2+3 z)} z+28 z-5 \sqrt{2} \sqrt{(2 z-1)(-2+3 z)}-8\right)^{-2}
\end{gathered}
$$

note que esta expressão só depende da variável $z$ e é sempre maior que zero para $0<z=$ $x \lambda<\frac{7-\sqrt{17}}{8}$ (mostre que ela não se anula neste intervalo e tome o valor em qualquer ponto).

Assim, o zero duplo do discriminante não ocorre em parâmetros admissíveis. Além disso, fixando $(\delta, \lambda)$, notamos que a mudança ocorre quando $\beta=\beta_{2}$. Agora, a mudança se dá em um ponto tal que $\delta \beta+\delta \lambda-1>0$. Assim, caso tomemos $\beta>\beta_{2}$, a inequação se mantém e portanto, para esses $\beta \mathrm{s} \mathcal{W}_{\beta}$ é vazia. Assim, a única configuração que nos interessa ocorre para $\beta<\beta_{2}$ e corresponde a figura 6.3 à esquerda.

Assim, temos duas curvas conexas: uma à esquerda que chamaremos de $\gamma_{1}$ e a outra à direita que chamaremos de $\gamma_{2}$. Antes de continuarmos, enunciemos um corolário:

Corolário 6.2.19. Fixados $(\delta, \lambda) \in \Delta$ e $\beta>0$ tal que $\delta \beta+\delta \lambda-1<0$, as curvas $\gamma_{1}$ e $\gamma_{2}$ são regulares 




Figura 6.4: Em vermelho a curva $\delta_{2} \lambda+\delta_{2} \beta_{2}-1$. Note que o gráfico tende a zero quando se aproxima de $\frac{7-\sqrt{17}}{8}$. De fato, ao substituirmos $z$ por $\frac{7-\sqrt{17}}{8}$, a equação se anula

Demonstração. No lema anterior, mostramos que os pontos nos quais $W_{x}, W_{y}$ e $W$ zeram simultaneamente ocorrem quando $\delta \beta+\delta \lambda-1 \geq 0$. Assim, quando $\delta \beta+\delta \lambda-1<0$, e $W=0$, uma das derivadas não se anula e pelo teorema da função implícita temos o resultado.

Agora, vamos restringir ainda mais as curvas de interesse:

Lema 6.2.20. Para $(\delta, \lambda) \in \Delta$ fixo e $\beta>0$ qualquer, a curva $\gamma_{1}$ não pertence a $\mathcal{S}_{\beta}$. Além disso, a curva $\gamma_{2}$ cruza em um único ponto a curva $M=0$. Este ponto é $\left(\frac{2 \delta}{1-\delta \beta}, \frac{-2(-1+\delta \beta+2 \lambda \delta)}{\delta^{2} \beta^{2}-2 \delta \beta+1}\right)$.

Demonstração. Comecemos notando que a curva $W=0$ cruza uma única vez com a $M=0$. De fato, de $M=0$, tiramos que $y=-\frac{x(-1+\lambda x)}{\delta}$. Substituindo na expressão de $W$, obtemos:

$$
W(y M)=-\frac{\delta^{2}(\delta \beta x-x+2 \delta)}{x^{5}(-1+\lambda x)^{2}}
$$

e portanto, $W$ só se anula sobre $M=0$ caso $x=\frac{2 \delta}{1-\delta \beta}$. Assim, para cada $(\delta, \lambda)$ e $\beta$ fixados, o cruzamento entre as duas curvas só é feito em um ponto. Agora, afirmo que a curva $\gamma_{2}$ é responsável por este cruzamento. De fato, comecemos notando, que a expressão de $y_{1}$ tende a infinito quanto $x$ tende a $\frac{2}{3 \lambda}$. Assim, se em algum momento, a curva $\gamma_{2}$ estiver abaixo da curva $M=0$, então, como $M=0$ é limitada em relação a $y$, essas curvas devem se cruzar. 
Agora, afirmo que para $x$ próximo a $\frac{1}{\lambda}$ a curva $\gamma_{2}$ deve ficar abaixo da curva $M=0$. De fato, ambas as curvas tendem a $y=0$ quando $x$ tende a $\frac{1}{\lambda}$, ou seja, elas tendem a um mesmo ponto. Assim, para que $\gamma_{2}$ fique abaixo da curva $M=0$, basta que o vetor tangente a ela, quando $x$ tende a $\frac{1}{\lambda}$, esteja mais próximo do eixo $x$ do que o vetor tangente de $M=0$ quando $x$ tende a $\frac{1}{\lambda}$. De fato, quando $x$ tende a $\frac{1}{\lambda}$ o vetor tangente de $M=0$ tende a $(\delta,-1)$. Já para o vetor tangente de $\gamma_{2}$ temos uma dificuldade extra, já que a sua norma tende a infinito. Assim, como só estamos interessados na direção do vetor, podemos normaliza-lo em cada ponto e tomar o seu limite. Tal vetor tende a direção: $(2 \delta,-1)$. Note que o vetor relativo a $\gamma_{2}$ está mais próximo do eixo $x$ do que o relativo a curva $M=0$ e portanto, $\gamma_{2}$ pertencea $M \geq 0$ para $x$ próximo de $\frac{1}{\lambda}$.

Desta forma, $\gamma_{2}$ deve curzar $M=0$ e, portanto, $\gamma_{1}$ não pode cruzar esta curva. Assim, $\gamma_{1}$ está totalmente forá de $M \geq 0$ ou totalmente dentro. Afirmo que está totalmente fora. De fato, note que ou $\gamma_{1}$ é vazio, ou está definido para $x$ suficientemente próximo a zero. Caso $\gamma_{1}$ seja vazio, não temos nada a fazer. Assim, vamos assumir $\gamma_{1}$ diferente de vazio. Quando tomamos a parametrização de $y_{1}$ e tomamos $x$ suficientemente próximo a zero, estamos na curva $\gamma_{1}$. Agora, $y_{1}$ tende a 1 quando $x$ tende a zero, o que significa que $\gamma_{1}$ fica tão próximo quanto se queira do ponto $(0,1)$ que está fora do conjunto $M \geq 0$. Assim, $\gamma_{1}$ deve estar fora de $M \geq 0$ e, consequentemente, fora de $\mathcal{S}_{\beta}$.

Assim, só temos interesse na curva $\gamma_{2}$. Tal curva cruza $M=0$ no ponto $\left(\frac{2 \delta}{1-\delta \beta}, \frac{-2(-1+\delta \beta+2 \lambda \delta)}{\delta^{2} \beta^{2}-2 \delta \beta+1}\right)$. Sabemos, portanto, que essa curva restrita a $M \geq 0$ é conexa. No entanto ainda precisamos estudar a restrição $A \geq 0$.

\subsubsection{Estudo da conexidade de $\gamma_{2}$ nos parâmetros admissíveis}

Nesta secção faremos um estudo da conexidade de $\gamma_{2}$ quando restrita aos parâmetros admissíveis. Em um primeiro momento, mostraremos que, ao fixar $(\delta, \lambda) \in \Delta$ e $\beta>0$ podemos perder a conexidade da curva. Em um segundo momento, mostraremos que a superfície $\mathcal{W}$ é conexa. 
Para começar o estudo, já mostramos que $\gamma_{2}$ cruza uma única vez com $M=0$ em $\mathcal{F}$. Assim, a parte de $\gamma_{2}$ restrita a $M \geq 0$ deve ser conexa. Resta estudar quando $\gamma_{2}$ pertence ao conjunto $A \geq 0$ :

Lema 6.2.22. Fixado $(\delta, \lambda) \in \Delta$ tal que $0<\lambda \delta<17-12 \sqrt{2}$, então existem $0<b_{2}(\delta, \lambda)<$ $b_{1}(\delta, \lambda)$ tais que caso $\left.\beta \in\right] b_{2}(\delta, \lambda), b_{1}(\delta, \lambda)\left[\right.$, então a curva $\gamma_{2}$ restrita a $\mathcal{S}_{\beta}$ tem duas componentes conexas distintas. Em quaisquer outros parâmetros, a curva $\gamma_{2}$ restrita a $\mathcal{S}_{\beta}$ é conexa.

Demonstração. Vamos começar procurando os pontos de encontro da curva $\gamma_{2}$ e de $A=0$. Com vista a esse objetivo, resolvemos a equação $A=0$ em função de $y$ e obtemos:

$$
y A=(\beta x+1)(1-\lambda x)
$$

substituindo $y$ por $y A$ na equação de $W$, obtemos:

$$
W(x, y A, \beta, \delta, \lambda)=-\frac{2 x^{2} \delta \beta^{2} \lambda-2 \lambda x^{2} \beta+3 x \delta \lambda \beta-x \delta \beta^{2}+\beta x-\delta \beta-1+\delta \lambda}{x^{2}(\beta x+1)^{3}(-1+\lambda x)^{3}}
$$

note que o denominador não se anula em $\mathcal{F}$ e portanto está bem definido. Assim, igualando $W(y A)$ a zero e resolvendo em relação a variável $x$ obtemos duas soluções:

$$
\begin{aligned}
& x_{1}=1 / 4 \frac{-\beta-3 \delta \lambda \beta+\delta \beta^{2}+\sqrt{\beta^{2}+6 \delta \beta^{2} \lambda-2 \delta \beta^{3}+\delta^{2} \lambda^{2} \beta^{2}+2 \delta^{2} \lambda \beta^{3}+\delta^{2} \beta^{4}-8 \lambda \beta+8 \delta \lambda^{2} \beta}}{\beta \lambda(\delta \beta-1)} \\
& x_{2}=-1 / 4 \frac{\beta+3 \delta \lambda \beta-\delta \beta^{2}+\sqrt{\beta^{2}+6 \delta \beta^{2} \lambda-2 \delta \beta^{3}+\delta^{2} \lambda^{2} \beta^{2}+2 \delta^{2} \lambda \beta^{3}+\delta^{2} \beta^{4}-8 \lambda \beta+8 \delta \lambda^{2} \beta}}{\beta \lambda(\delta \beta-1)}
\end{aligned}
$$

Caso ambos sejam reais, temos $x_{1}<x_{2}$.

A primeira coisa que devemos nos preocupar é saber quando que essas raízes são reais. Assim, comecemos estudando o radicando de $x_{1}$ e $x_{2}$ :

$$
\beta(\delta \lambda-1+\delta \beta)\left(\delta \lambda \beta+8 \lambda-\beta+\delta \beta^{2}\right)
$$

assim, podemos notar que $\beta>0$ por causa de nossas restrições. Além disso, podemos assumir que $\delta \lambda-1+\delta \beta<0$. De fato, para que $\mathcal{S}_{\beta}$ seja não vazio, devemos ter $\frac{\delta}{1-\delta \beta}<\frac{1}{\lambda}$, ou seja, $\delta \lambda<1-\delta \beta$ o que implica que, de fato:

$$
0<1-\delta \beta-\delta \lambda
$$


e temos a afirmação. Assim, para que o radicando seja positivo, devemos ter:

$$
\delta \lambda \beta+8 \lambda-\beta+\delta \beta^{2}<0
$$

resolvendo essa expressão em função de $\beta$ obtemos duas soluções:

$$
\begin{aligned}
& b_{1}=\frac{1}{2} \frac{-\delta \lambda+1+\sqrt{\delta^{2} \lambda^{2}-34 \delta \lambda+1}}{\delta} \\
& b_{2}=\frac{1}{2} \frac{-\delta \lambda+1-\sqrt{\delta^{2} \lambda^{2}-34 \delta \lambda+1}}{\delta}
\end{aligned}
$$

onde o radicando destas expressões vale: $\delta^{2} \lambda^{2}-34 \delta \lambda+1$. Para que $b_{1}$ e $b_{2}$ sejam reais, devemos ter $\delta^{2} \lambda^{2}-34 \delta \lambda+1>0$, além disso, para os parâmetros serem válidos devemos ter $\delta \lambda>0$. Assim:

$$
0<\lambda \delta<17-12 \sqrt{2}
$$

$\mathrm{Ou}$

$$
\lambda \delta>17+12 \sqrt{2}
$$

Agora, para que $0<1-\delta \beta-\delta \lambda$, como $\beta>0$, devemos ter $\delta \lambda<1$ e portanto:

$$
0<\lambda \delta<17-12 \sqrt{2}
$$

com essas restrições, temos que $b_{1}>0$ e $b_{2}>0$. Além disso, para todo $\beta \in\left[b_{2}, b_{1}\right]$, o radicando de $x_{1}$ e $x_{2}$ é maior ou igual a zero. Isso implica que $x_{1}$ e $x_{2}$ são reais. Ainda é necessário saber se esses valores de $x$ são admissíveis, ou seja, se são maiores do que zero e menores do que $\frac{1}{\lambda}$.

Munido das desigualdades: $0<\lambda \delta<17-12 \sqrt{2}, 0<1-\delta \beta-\delta \lambda$ e do fato de nossos parâmetros serem positivos, pode-se concluir que $x_{1}$ e $x_{2}$ são sempre positivos e menores do que $\frac{1}{\lambda}$. Além disso, também podemos concluir que $\frac{\delta}{1-\delta \beta}<x_{1}<x_{2}$. Isso é especialmente importante, pois garante que $x_{1}$ e $x_{2}$ não podem ser descartados, já que são maiores do o valor no qual as curvas $A=0$ e $M=0$ se cruzam. Desta forma, existem $y 1$ e $y 2$ tais que $\left(x_{1}, y 1\right)$ e $\left(x_{2}, y 2\right)$ pertencem a $\mathcal{W S}$ e $A\left(x_{1}, y 1\right)=0=A\left(x_{2}, y 2\right)$.

Agora, notemos que os pontos $\left(x_{1}, y 1\right)$ e $\left(x_{2}, y 2\right)$ pertencem ao ramo $\left(x, y_{2}, \beta, \delta, \lambda\right)$ (lembremos das soluções de $W=0$ ). Assim, a parte da curva cuja imagem de $A$ muda de sinal 


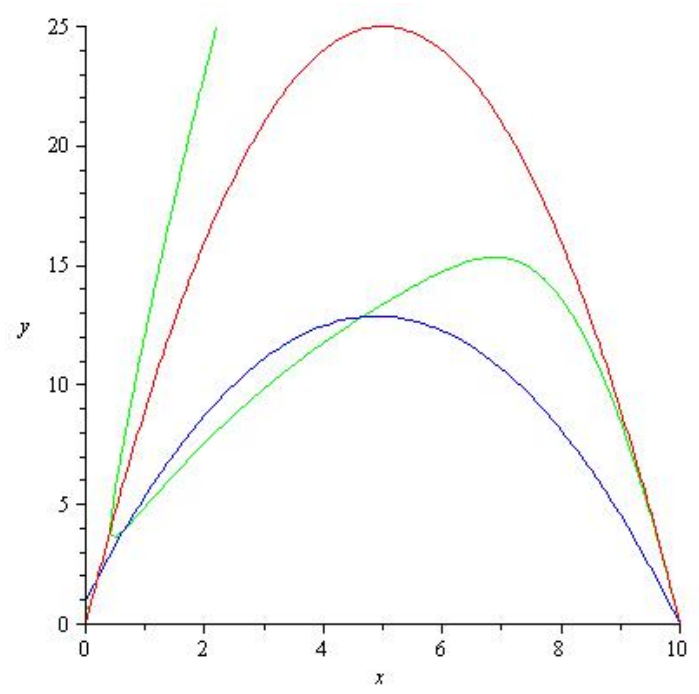

Figura 6.5: Em vermelho a curva $M=0$, em azul a curva $A=0$ e em verde a curva $W=0$. Note como a intersecção de $W=0 \operatorname{com} \mathcal{S}_{\beta}$ é não conexa. A figura foi obtida com $\delta=0.1$, $\lambda=0.1, \beta=\frac{99}{20}$

esta contida neste ramo e portanto é limitada. Como $y_{1}$ tende a infinito quando $x$ tende a $\frac{2}{3 \lambda}$ pela esquerda, o ramo $\left(x, y_{1}, \beta, \delta, \lambda\right)$ está contido no conjunto $A \geq 0$. Assim, essa parte de $y_{2}$ cuja imagem em $A$ mudou de sinal, ficou negativa e, portanto, fora dos parâmetros admissíveis.

Assim, caso $x_{2} \neq x_{1}$, ou seja, $b_{1} \neq b_{2}$, a curva $\gamma_{2}$ sai dos parâmetros admissíveis em $\left(x_{2}, y_{2}\right)$ e volta em $\left(x_{1}, y_{1}\right)$ (esses pontos ainda são admissíveis). Desta forma, para $\left.\beta \in\right] b_{2}, b_{1}[$, a curva $\gamma_{2}$ possui duas componentes conexas.

Queremos mostrar que $\mathcal{W S}$ é conexo. Para isso, vamos demostrar um resultado preliminar:

Lema 6.2.23. Seja I um intervalo conexo de $\mathbb{R}$. Suponha que, para todo $p \in I$, exista uma curva $\Gamma_{p}$ em $\mathbb{R}^{2}$ contínua, conexa e não fechada, onde sua fronteira vale $f(p)$ e $g(p)$. Suponha que a função $f$ ou a função g seja contínua. Nesse caso o conjunto $J=\cup_{p \in I} p \times \Gamma_{p}$ é conexo em $\mathbb{R}^{3}$

Demonstração. Suponha por absurdo que $J$ não seja conexo. Então existem $U$ e $V$ abertos 
de $\mathbb{R}^{3}$ que desconectam $J$. Como $U \cap J \neq \emptyset$, existe $x=\left(p_{0}, q_{0}\right) \in U$ que pertence a $J$. Agora, notemos que, $p \times \Gamma_{p}$ é um conjunto conexo de $\mathbb{R}^{3}$. Assim, $U$ deve conter $p_{0} \times \Gamma_{p_{0}}$. Suponha que $f$ seja contínua (demonstração é análoga caso seja $g$ ). Nesse caso, a curva $(p, f(p))$ é conexa em $\mathbb{R}^{3}$. Como $U$ contém $\left(p_{0}, f\left(p_{0}\right)\right)$, ele deve conter a curva $(p, f(p))$. Então, ele contém $\cup_{p \in I} p \times \Gamma_{p}=J$. Logo $V$ e $U$ se intersectam e temos um absurdo. Logo $J$ é conexo.

Assim, estamos em condições de mostrar que:

Proposição 6.2.24. Fixado $(\delta, \lambda) \in \Delta$, a superfície $\mathcal{W S}$ é conexa.

Demonstração. Para isso, comecemos notando que para $\delta \lambda \geq 17-12 \sqrt{2}$, fixado $\beta, \mathcal{W S}_{\beta}$ é conexo. Além disso, uma vez que as funções $M$ e $W$ são contínuas, sabemos que a fronteira de $\mathcal{W} \mathcal{S}_{\beta}$ varia continuamente em relação a $\beta$. Desta forma, estamos nas condições do lema anterior e temos a conexidade.

Agora, caso $\delta \lambda<17-12 \sqrt{2}$ notemos que:

$$
W(x, y A, 0, \delta, \lambda)=\delta \lambda-1
$$

que é sempre diferente de zero. Assim, para $(\delta, \lambda)$ fixados e $\beta$ suficientemente próximo de zero, $W(y A)$ não zera para nenhum $x$. Logo, $b_{2}>0$ e para todo $\beta<b_{2}, W(y A)$ não se anula para nenhum valor de $x$ válido. Tal curva só deixará de ser conexa quando $\beta \in] b_{2}, b_{1}[$.

Agora, notemos que, para $\beta \in] b_{2}, b_{1}$ [ fixado, a curva $\mathcal{W} \mathcal{S}_{\beta}$ tem duas componentes conexas. Chamaremos a que fica mais a esquerda de de $C 1_{\beta}$ e a outra de $C 2_{\beta}$. Pelo menos a fronteira de um dos lados de $C_{1}$ e $C_{2}$ permanece contínua devido a continuidade da função $M$. Assim, em ambos estamos nas condições do lema anterior. Assim sendo, $\cup_{\beta \in] b_{2}, b_{1}[} C 1_{\beta}$ e $\cup_{\beta \in] b_{2}, b_{1}[} C 2_{\beta}$ são conjuntos conexos. Pelo mesmo argumento, tomando: $\left(\cup_{\beta \in] b_{2}, b_{1}[} C 1_{\beta}\right) \cup\left(\cup_{\left.\beta \in] 0, b_{2}\right] \cup\left[b_{1}, \infty[\right.} \mathcal{W} \mathcal{S}_{\beta}\right)$ e $\left(\cup_{\beta \in] b_{2}, b_{1}[} C 2_{\beta}\right) \cup\left(\cup_{\left.\beta \in] 0, b_{2}\right] \cup\left[b_{1}, \infty[\right.} \mathcal{W} \mathcal{S}_{\beta}\right)$ concluímos que eles são conjuntos conexos. Além disso, pela primeira observação, ambos os conjuntos tem intersecção não vazia. Assim sendo, $\mathcal{W S}$ é conexo.

Reunimos esses resultados no seguinte teorema: 
Teorema 6.2.25. Para todo $(\delta, \lambda) \in \Delta$ fixo, $\mathcal{W S}$ é uma superfície regular e conexa. No entanto, se também fixarmos $\beta \in] b_{1}, b_{2}\left[\right.$, então a intersecção de $W=0$ e $\mathcal{S}_{\beta}$ é não conexa.

\subsubsection{Estudo sobre a dobra geral}

Com os resultados já provados, somos capazes de tratar da superfície de dobra geral (ver capítulo 4). No capítulo 4, mostramos que ela é formado por uma superfície imersa $S N_{1}$ e por uma curva mergulhada $S N_{2}$. Para que $S N_{1}$ seja mergulhada, mostramos que basta a superfície de dobras não se auto-intersectar. Dessa forma, seria interessante provar:

- a superfície de dobras geral é conexa.

- a superfície de dobras geral não se auto-intersecta.

A primeira condição é direta do último teorema:

Corolário 6.2.27. Para todo $(\delta, \lambda) \in \Delta$ fixo, a dobra geral ( $S N_{1}$ e suas degenerescências) é conexa em $\mathcal{W}_{1}$. Note que $\mathcal{W}_{1}$ nada mais é do que $\mathcal{W}$ com $\beta>0$ e portanto, temos o resultado em $\mathcal{W}$ para $\beta$ positivo.

Demonstração. Fixado $(\delta, \lambda) \in \Delta$, sabemos que a superfície $\mathcal{W S}$ é conexa e regular. Assim, como $\Psi=(A, M)$ é contínua, temos que o gráfico $g$ de $\Psi_{\mid \mathcal{W S}}$ é um conjunto conexo. Definindo $\Pi$ como sendo a projeção de $\mathbb{R}^{2} \times \mathcal{W}_{1}$ em $\mathcal{W}_{1}$, temos que $\Pi$ é contínua e portanto $\Pi(g)$ é um conjunto conexo. Agora, $\Pi(g)$ corresponde à dobra geral e concluímos o resultado.

Agora, queremos mostrar que a dobra geral não se auto-intersecta. Isso é equivalente a mostrar que não existe $(\alpha, \beta, \mu, \delta, \lambda)$ onde a função $F(x)$ possui duas raízes $\left(x_{1}\right.$ e $\left.x_{2}\right)$ distintas de multiplicidade dois válidas. Para provar isso vamos assumir por absurdo que existem essas duas raízes e chegar a uma contradição. Assim, supondo que existam tais $x_{1}$ e $x_{2}$, podemos provar que:

Lema 6.2.28. Para $\beta>0, x_{1}+x_{2}<\frac{1}{2 \lambda}$.

Demonstração. Tome o seguinte polinômio:

$$
\alpha^{2} \mu \lambda\left(x-x_{1}\right)^{2}\left(x-x_{2}\right)^{2}\left(x-x_{3}\right)=Q(x)
$$


Imaginar que $F(x)$ possui duas raízes de multiplicidade dois é imaginar que $F(x)$ é igual a $Q(x)$ para algum $(\alpha, \beta, \mu, \delta, \lambda) \in \mathcal{W}_{1} \times \Delta$. Assim, para que isso seja verdade, o polinômio $F(x)-Q(x)$ tem que ser igual a zero para qualquer $x$, ou seja, todos os seus coeficientes devem se anular. Assim, para procurar possíveis parâmetros onde isso possa ocorrer devemos resolver o sistema formado pelos coeficientes de $F(x)-Q(x)$ igualados a zero. Isso gera cinco equações. Do coeficiente livre de $x$ obtemos:

$$
x_{3}=\frac{\mu+\delta}{\alpha^{2} \mu \lambda x_{1}^{2} x_{2}^{2}}
$$

Aplicando ao coeficiente de $x^{4}$ obtemos a seguinte equação:

$$
-x_{1}{ }^{2} \mu x_{2}{ }^{2}\left(2 \lambda x_{2}+2 x_{1} \lambda-1\right) \alpha^{2}-2 \alpha \mu \lambda \beta x_{1}{ }^{2} x_{2}{ }^{2}-\mu-\delta=0
$$

Como $\alpha$ é positivo, para que essa equação possa se anular, devemos ter:

$$
2 \lambda x_{2}+2 x_{1} \lambda-1<0
$$

e portanto:

$$
x_{1}+x_{2}<\frac{1}{2 \lambda}
$$

Uma maneira de prosseguir no estudo seria mostrar que a função $Q(x)$ definida na demonstração anterior nunca pode ser igual a $F(x)$. No entanto, resolver o sistema necessário para isso é tarefa difícil. Assim, vamos proceder de uma outra forma. Como $x_{1}+x_{2}<\frac{1}{2 \lambda}$ e $x_{1} \neq x_{2}$, isso significa que, sem perda de generalidade, $x_{1}<\frac{1}{4 \lambda}$. A estratégia é encontrar parâmetros onde esses pontos não são válidos.

Lema 6.2.29. Caso $\lambda \delta>\frac{1}{7}$, não existe auto-interseç̧ão da superfície de dobra em $\mathcal{W}_{1}$.

Demonstração. Para obter esse resultado, vamos fazer uma análise da função disc previamente definida e delimitar os casos onde existe $x<\frac{1}{4 \lambda}$ válido. Fazendo a mudança de coordenadas $x \lambda=z$ essa função passa a valer:

$$
\begin{gathered}
(z-1)^{2}\left(\left(\delta^{2} \beta^{2}+16 z^{2}-10 \delta \beta+z^{2} \beta^{2} \delta^{2}-24 z+26 z \beta \delta-2 z \beta^{2} \delta^{2}-16 z^{2} \beta \delta+9\right) x^{2}+\right. \\
\left.\left(-32 z^{2} \delta+4 z^{2} \beta \delta^{2}-20 \delta+52 z \delta+4 \delta^{2} \beta-8 z \beta \delta^{2}\right) x+4 z^{2} \delta^{2}-8 z \delta^{2}+4 \delta^{2}\right)
\end{gathered}
$$






Figura 6.6: Em azul a curva $\frac{z \delta}{x 2}$ e em vermelho $\frac{z \delta}{x 1}$.

Resolvendo essa expressão em $x$ obtemos:

$$
\begin{aligned}
& X 1=2 \frac{\left(8 z-z \beta \delta+\delta \beta-5+2 \sqrt{12 z^{2}-14 z+4}\right) \delta(z-1)}{16 z^{2}-24 z+9-10 \delta \beta+\delta^{2} \beta^{2}-2 z \beta^{2} \delta^{2}+26 z \beta \delta+z^{2} \beta^{2} \delta^{2}-16 z^{2} \beta \delta}, \\
& X 2=2 \frac{\left(8 z-z \beta \delta+\delta \beta-5-2 \sqrt{12 z^{2}-14 z+4}\right) \delta(z-1)}{16 z^{2}-24 z+9-10 \delta \beta+\delta^{2} \beta^{2}-2 z \beta^{2} \delta^{2}+26 z \beta \delta+z^{2} \beta^{2} \delta^{2}-16 z^{2} \beta \delta} .
\end{aligned}
$$

Tomando $\beta=0$, temos:

$$
\begin{aligned}
& x 1=2 \frac{\left(8 z-5+2 \sqrt{12 z^{2}-14 z+4}\right) \delta(z-1)}{9+16 z^{2}-24 z}, \\
& x 2=2 \frac{\left(8 z-5-2 \sqrt{12 z^{2}-14 z+4}\right) \delta(z-1)}{9+16 z^{2}-24 z} .
\end{aligned}
$$

Assim, tomando $\frac{z \delta}{x 1}$ e $\frac{z \delta}{x 2} \operatorname{com} x \lambda=z<\frac{1}{4}$ obtemos a figura 6.6.

Para prosseguir a análise, devemos interpretar o gráfico. Nele fixamos $\beta=0$. O contradomínio das funções corresponde a multiplicação entre $\delta$ e $\lambda$. Assim, fixado $\left(\delta_{0}, \lambda_{0}\right) \in \Delta$, devemos fixar $\delta_{0} \lambda_{0}$ na imagem e observar quais pontos no domínio são levados a ele.

Fazendo a análise da função discriminante, chegamos a conclusão que entre os dois gráficos $\frac{z \delta}{x 1}$ e $\frac{z \delta}{x 2}$ o discriminante é negativo. Além disso, fixado $\lambda \delta$ a componente acima de $\frac{z \delta}{x 1}$ corresponde aos pontos da curva $\gamma_{1}$ e, portanto, podem ser descartados. Isso implica que, para existir $x \lambda<\frac{1}{4}$ válido com $\beta=0$, devemos estar abaixo do gráfico $\frac{z \delta}{x 2}$ e, para isso acontecer, devemos ter $\delta \lambda<\frac{1}{7}$. 
Quando tomamos $\beta>0$, notemos que $X 2>x 2$. Dessa forma, $\frac{z \delta}{X 2}<\frac{z \delta}{x 2}$ e portanto, fixando $\beta$ positivo, o máximo da função $\frac{z \delta}{X 2}$ em $0 \leq z \leq \frac{1}{4}$ é menor do que da função $\frac{z \delta}{x 2}$. Assim, fixado $(\delta, \lambda)$, se não existir $x<\frac{1}{4 \lambda}$ válido para $\beta=0$, não existirá para $\beta>0$.

Dessa forma, caso $\delta \lambda \geq \frac{1}{7}$, então não existe $x<\frac{1}{4 \lambda}$ válido. Assim, a dobra geral não se auto-intersecta.

Esse resultado ainda pode ser melhorado caso consideremos diretamente a restrição da função $M$.

Lema 6.2.30. Caso $\lambda \delta>\frac{1}{8}$ não existe auto-intersecção da superfície de dobra em $\mathcal{W}_{1}$.

Demonstração. Considerando que $\delta \lambda<\frac{1}{7}, x \lambda<\frac{1}{4}$ e a função $M$ chegamos a conclusão de que, para $M$ ser maior do que zero devemos ter:

$$
x>\frac{2 \delta}{1-\delta \beta}
$$

Assim, temos que:

$$
\frac{1}{4}>x \lambda>\frac{2 \delta \lambda}{1-\delta \beta}
$$

o que implica que:

$$
1-\delta \beta-8 \delta \lambda>0
$$

e portanto $\delta \lambda<\frac{1}{8}$.

Isso encerra os resultados sobre a dobra geral. Note que, como não pedimos que $x 1 \neq x 2$ e, portanto, em particular mostramos que não existe raiz quarta de $F(x)$ para $\beta>0$ e $\delta \lambda>\frac{1}{8}$.

\subsection{Estudo dos equilíbrios não hiperbólicos nos quais traço $=0$}

Uma outra forma de um equilíbrio deixar de ser hiperbólico é o traço ser igual a zero e o determinante ser positivo. Em um primeiro momento, não vamos nos preocupar com o sinal 
do determinante, mas apenas com os pontos que anulam a função traço.

Assim estamos interessados no traço do linearizado do sistema 1.1:

\section{Definição 6.3.1.}

$$
\begin{aligned}
& \begin{array}{llll}
t r: & \mathcal{F} & \rightarrow & \mathbb{R}
\end{array} \\
& (x, y, \beta, \delta, \lambda) \mapsto \operatorname{traco}\left(\mathcal{J}_{x, y}\left(f_{1}, f_{2}\right)(x, y, A((x, y, \beta, \delta, \lambda)), \beta, M((x, y, \beta, \delta, \lambda)), \delta, \lambda)\right)
\end{aligned}
$$

onde $\mathcal{J}$ é a matriz Jacobiano. Portanto:

$$
t r=\left((2+\delta-3 \lambda x) y+\left(2 \lambda x^{2}-\lambda^{2} x^{3}-x\right) \beta-x+4 \lambda x-2 \lambda^{2} x^{2}-2+\lambda x^{2}\right) y^{-1}
$$

Note que o denominador de $t r$ é sempre diferente de zero em $\mathcal{F}$ e, portanto, esta função está bem definida. Além disso, ela é linear em y e $\beta$, sendo o coeficiente de $\beta$ igual a $-x(-1+\lambda x)^{2}$. Assim, caso fixemos $(\delta, \lambda) \in \Delta$ e $0<x<\frac{1}{\lambda}$, a curva $t r=0$ é uma reta no plano $(y, \beta)$. Desta forma, fixado $(\delta, \lambda) \in \Delta$, a superfície $t r=0$ é regrada.

Aproveitando esta interpretação, começamos nos perguntando, para quais $x \in] 0, \frac{1}{\lambda}[$ a reta $t r=0$ é não vazia.

Lema 6.3.2. Fixado $(\delta, \lambda) \in \Delta$, a curva $t r=0$ para $x$ fixo é nãa vazia caso $x \lambda<\frac{2+\delta}{3}$. Caso $\delta \geq 1$, então para qualquer $x$, a curva é não vazia. Além disso, fixado $(\delta, \lambda) \in \Delta e$ resolvendo $t r=0$ em função de $\beta$ encontramos uma um expressão analítica que chamaremos de $\beta_{t r}$.

Para provar o lema, utilizaremos a seguinte função:

Definição 6.3.3. Chamaremos de $\beta_{\text {tr }}(x, y, \delta, \lambda)$ o único valor de $\beta$ em $(x, y, \delta, \lambda)$ tal que $\operatorname{tr}\left(x, y, \beta_{t r}(x, y, \delta, \lambda), \delta, \lambda\right)=0$. Esta função é igual a:

$$
\beta_{t r}(x, y, \delta, \lambda)=-\frac{-2 y-\delta y+3 \lambda x y+2+x-4 \lambda x+2 \lambda^{2} x^{2}-\lambda x^{2}}{x(-1+\lambda x)^{2}}
$$

Demonstração. Note que o denominador de $\beta_{t r}$ é positivo em $\mathcal{F}$ e, portanto, está bem definida e, como o numerador é um polinômio, essa função é analítica em $\mathcal{F}$. Além disso, o sinal de $\beta_{t r}$ só depende do numerador. Tomando o numerador, obtemos:

$$
y(2+\delta-3 \lambda x)-2(1-\lambda x)^{2}-x(1-\lambda x)
$$




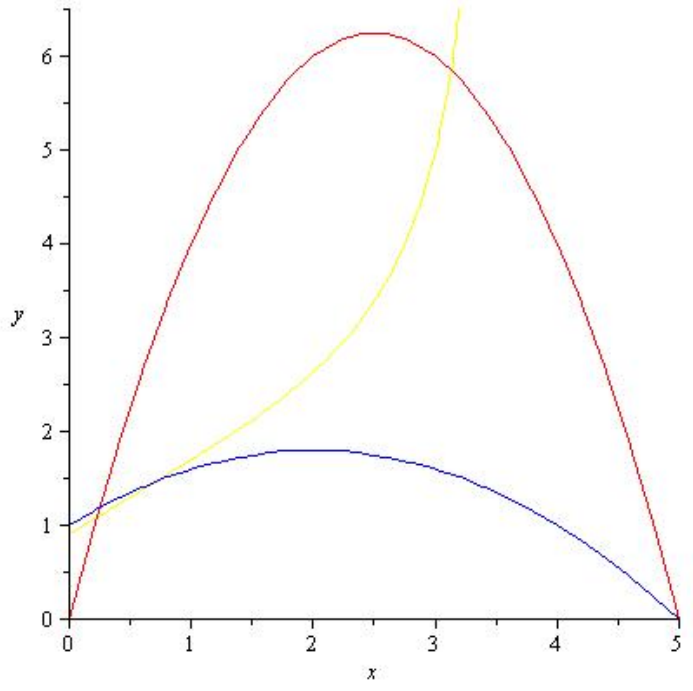

Figura 6.7: Em vermelho a curva $M=0$, em azul a curva $A=0$ e em amarelo a curva $\operatorname{tr}=0$. A figura foi obtida $\operatorname{com} \delta=0.2, \lambda=0.2, \beta=1$

note que o único termo que pode ser positivo é o primeiro. Como $y>0$, precisamos ter $2+\delta-3 \lambda x>0$, ou seja, $x \lambda<\frac{2+\delta}{3}$. Desta forma, existe um $y$ suficientemente grande, tal que $\beta_{t r}$ fique positivo. Caso contrário, $\beta_{t r}$ é sempre negativo e estamos fora dos parâmetros admissíveis.

Agora, estamos interessados em ter uma ideia geométrica a respeito da superfície $t r=0$.

Lema 6.3.4. A curva tr $=0$ é contínua e conexa.

Demonstração. Fixado $(\delta, \lambda) \in \Delta$ e $\beta>0$, restringindo $x$ a $] 0, \frac{2+\delta}{3 \lambda}[$ (lema anterior) e $\operatorname{tr}$ é linear em $y$, podemos resolver $t r=0$ em função de $y$, obtendo, para cada $x$ um único $y(x)$. Assim, a curva $t r=0$ passa a ser representada pelo gráfico $(x, y(x)), \operatorname{com} x \in] 0, \frac{2+\delta}{3 \lambda}[$. Como tr é contínua, essa curva é contínua e conexa.

\subsection{Estudo dos equilíbrios hiperbólicos}

Agora que já analisamos as curvas det $=0$ e $t r=0$, estamos prontos para tratar dos equilíbrios hiperbólicos. Com esse intuito, vamos fixar $(\delta, \lambda) \in \Delta$ e $\beta>0$ e tratar dos 


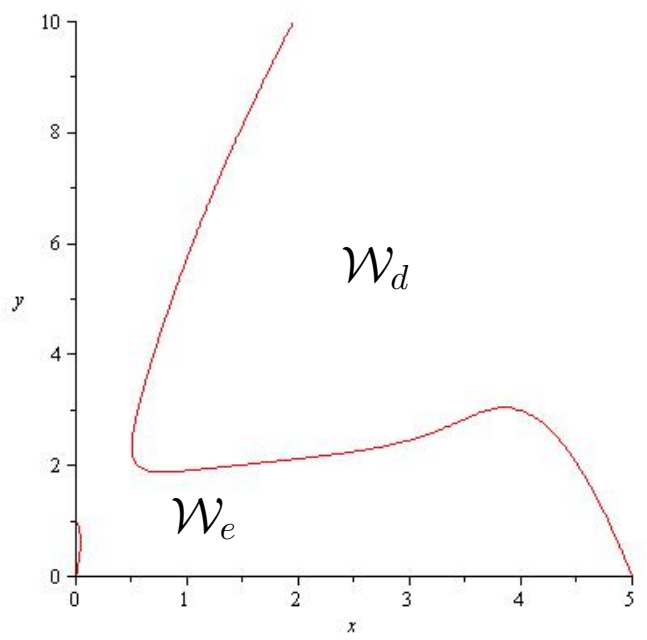

Figura 6.8: A esquerda temos $\mathcal{W}_{e}$ e a direita temos $\mathcal{W}_{d}$. Para o desenho, fixamos $\lambda=0.2, \delta=0.2$ e $\beta=1$.

equilíbrios em $\mathcal{S}_{\beta}$. Neste caso, como estamos interestados no sinal do determinante do linearizado do campo, calculado no equilíbrio, não podemos continuar a conta com $W$, pelo menos em um primeiro momento. Assim, notemos que:

$$
\left(\left(f_{1}\right)_{x}\left(f_{2}\right)_{y}-\left(f_{1}\right)_{y}\left(f_{2}\right)_{x}\right)(x, y, A(x, y, \beta, \delta, \lambda), \beta, M(x, y, \beta, \delta, \lambda), \delta, \lambda)=W 2
$$

e

$$
\frac{W 2}{W}=-y x^{3}(-1+\lambda x)^{2}<0
$$

Logo o sinal do determinante do linearizado do campo é o oposto ao de $W$. Assim, podemos continuar a trabalhar com $W$, mas sempre levando em conta uma mudança de sinal.

Vamos dividir $\mathcal{F}$ em duas componentes dependendo da curva $\gamma_{2}$ (uma das componentes conexas de $W=0$ ). Temos: uma componente à esquerda que chamaremos de $\mathcal{W}_{e}$ e outra à direita que chamaremos de $\mathcal{W}_{d}(6.8)$. Com esta divisão, obtemos:

Lema 6.4.1. Caso estejamos sobre parâmetros válidos, em $\mathcal{W}_{e}$ temos nós e focos, enquanto em $\mathcal{W}_{d}$ temos selas.

Demonstração. Pela continuidade de $W$, basta tomar um ponto de $\mathcal{W}_{e}$ e um ponto de $\mathcal{W}_{d}$ para decidirmos o sinal de toda a região. Assim, sendo, fixemos $x=\frac{4}{5 \lambda}$. Neste ponto, existe 
apenas um único $y_{0}>0$ tal que $\left(\frac{4}{5 \lambda}, y_{0}\right) \in\{W=0\}$. Pelas análises feitas sobre $W=0$, sabemos que:

$y_{0}=\frac{1}{50}\left(-2 \delta \beta+2-5 \lambda \delta+\sqrt{\frac{140 \lambda \delta+4+25 \lambda^{2} \delta^{2}+4 \delta^{2} \beta^{2}+56 \delta \beta+20 \lambda \beta \delta^{2}}{\lambda^{2}}} \lambda\right) \lambda^{-1} \delta^{-1}$

e que:

$$
W\left(\frac{4}{5 \lambda}, y, \beta, \delta, \lambda\right)=1 / 32 \frac{\left(50 y \delta \beta \lambda-16 \beta+125 \delta y \lambda^{2}-40 \lambda+625 \delta y^{2} \lambda^{2}-50 y \lambda\right) \lambda}{y^{3}}
$$

Assim, vamos tomar $0<y^{\prime}<y_{0}$ e $y_{0}<y^{\prime \prime}$ :

$$
\begin{aligned}
& y^{\prime}=\frac{1}{50}\left(-2 \delta \beta+1-5 \lambda \delta+\sqrt{\frac{140 \lambda \delta+4+25 \lambda^{2} \delta^{2}+4 \delta^{2} \beta^{2}+56 \delta \beta+20 \lambda \beta \delta^{2}}{\lambda^{2}}} \lambda\right) \lambda^{-1} \delta^{-1} \\
& y^{\prime \prime}=\frac{1}{50}\left(-2 \delta \beta+3-5 \lambda \delta+\sqrt{\frac{140 \lambda \delta+4+25 \lambda^{2} \delta^{2}+4 \delta^{2} \beta^{2}+56 \delta \beta+20 \lambda \beta \delta^{2}}{\lambda^{2}}} \lambda\right) \lambda^{-1} \delta^{-1}
\end{aligned}
$$

Desta forma, temos:

$$
\begin{aligned}
& \left(\frac{4}{5 \lambda}, y^{\prime}\right) \in \mathcal{W}_{e} \\
& \left(\frac{4}{5 \lambda}, y^{\prime \prime}\right) \in \mathcal{W}_{d}
\end{aligned}
$$

e como:

$$
\begin{gathered}
W\left(\frac{4}{5 \lambda}, y^{\prime}\right)=-1 / 4 \lambda\left(-1+2 \sqrt{\frac{140 \lambda \delta+4+25 \lambda^{2} \delta^{2}+4 \delta^{2} \beta^{2}+56 \delta \beta+20 \lambda \beta \delta^{2}}{\lambda^{2}}} \lambda\right) \delta^{-1}<0 \\
W\left(\frac{4}{5 \lambda}, y^{\prime \prime}\right)=1 / 4 \lambda\left(1+2 \sqrt{\frac{140 \lambda \delta+4+25 \lambda^{2} \delta^{2}+4 \delta^{2} \beta^{2}+56 \delta \beta+20 \lambda \beta \delta^{2}}{\lambda^{2}}} \lambda\right) \delta^{-1}>0
\end{gathered}
$$

temos que: os equilíbrios em $\mathcal{W}_{e}$ são nós ou focos e os equilíbrios em $\mathcal{W}_{d}$ são selas.

Agora vamos estudar o sinal do traço nestes equilíbrios. Caso o traço seja positivo, então, como estamos em $\mathbb{R}^{2}$ podemos ter uma sela ou um repulsor. Caso o traço seja negativo, temos uma sela ou um atrator. Assim, começamos notando que a curva $\operatorname{tr}=0$ divide $\mathcal{F}_{\beta, \delta, \lambda}$ em duas componentes conexas. Chamaremos de $\mathcal{T}_{e}$ a que fica à esquerda e de $\mathcal{T}_{d}$ à que fica a direita da curva (6.9). Assim, obtemos o seguinte resultado:

Lema 6.4.2. Em $\mathcal{T}_{e}$ o traço é positivo e em $\mathcal{T}_{d}$ o traço é negativo.

Demonstração. Para provar isso, notemos que, por causa da continuidade de $t r$, basta tomar um único ponto de cada região e calcular o seu sinal com relação a tr. Assim, lembremos que:

$$
0<x<\frac{2+\delta}{3 \lambda}
$$




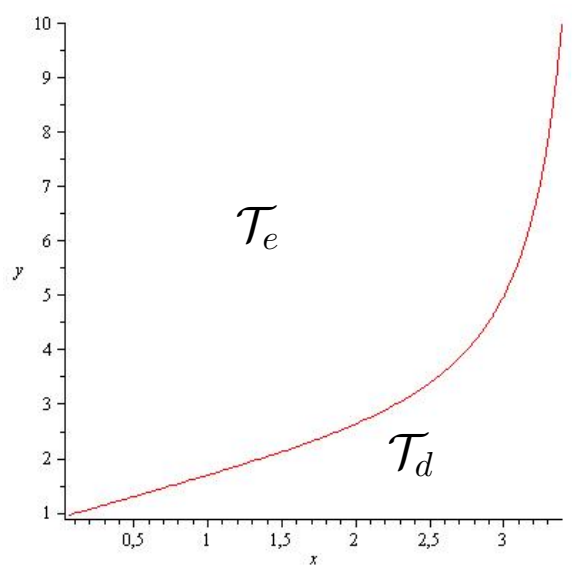

Figura 6.9: A esquerda temos $\mathcal{T}_{e}$ e a direita temos $\mathcal{T}_{d}$. Para o desenho, fixamos $\lambda=0.2, \delta=0.2 \mathrm{e}$ $\beta=1$.

para que exista $(x, y)$ no quadrante positivo tal que $\operatorname{tr}(x, y)=0$. Assim, $x=\frac{2}{3 \lambda}$ é sempre válido pois $\delta>0$. Assim, para que $\operatorname{tr}\left(\frac{2}{3 \lambda}, y_{0}\right)=0$, devemos ter:

$$
y_{0}=\frac{2}{27} \frac{3 \lambda+\beta+3}{\lambda \delta}
$$

Tomemos $0<y^{\prime}<y_{0}$ e $y_{0}<y^{\prime \prime}:$

$$
\begin{gathered}
y^{\prime}=2 / 9 \frac{\lambda+1}{\lambda \delta} \\
y^{\prime \prime}=\frac{2}{27} \frac{3 \lambda+2 \beta+3}{\lambda \delta}
\end{gathered}
$$

Substituindo em $t r$, obtemos:

$$
\begin{gathered}
\operatorname{tr}\left(\frac{2}{3 \lambda}, y^{\prime}\right)=-\frac{2}{27} \frac{\beta}{\lambda}<0 \\
\operatorname{tr}\left(\frac{2}{3 \lambda}, y^{\prime \prime}\right)=\frac{2}{27} \frac{\beta}{\lambda}>0
\end{gathered}
$$

Assim sendo, em $\mathcal{T}_{e}$ temos o traço positivo e em $\mathcal{T}_{d}$ o traço é negativo. 


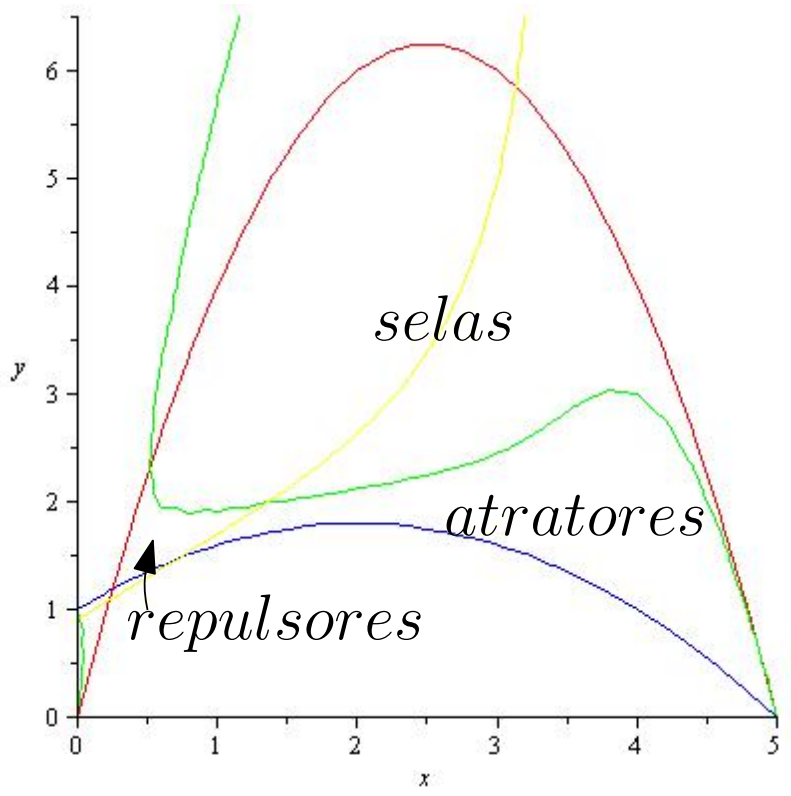

Figura 6.10: Em azul a cruva $A=0$, em vermelho $M=0$, em verde $W=0$ e em amarelo $t r=0$. Para o cálculo deste gráfico, tomamos $\delta=0.2, \lambda=0.2$ e $\beta=1$. Note que temos quatro regiões em $\mathcal{S}$ : duas são de selas, uma de atratores e a outra de repulsores. 


\section{Capítulo 7}

\section{Estudo das bifurcações de Bogdanov-Takens}

Neste capítulo vamos estudar os pontos de bifurcação do tipo Bogdanov-Takens (ver capítulo 10 para mais informações) do sistema 1.1. Queremos fazer um estudo com o mínimo de calculo numérico que nos for permitido, usando procedimentos simples de álgebra e análise clássica.

Começamos definindo o objeto de estudo:

Definição 7.0.3. Chamaremos $\left(x_{0}, y_{0}, \alpha_{0}, \beta_{0}, \mu_{0}, \delta_{0}, \lambda_{0}\right) \in \mathbb{R}^{+} \times \mathbb{R}^{+} \times \mathcal{W} \times \Delta$ de candidato a bifurcação de Bogdanov-Takens caso $\left(x_{0}, y_{0}\right)$ com os parâmetros $\left(\alpha_{0}, \beta_{0}, \mu_{0}, \delta_{0}, \lambda_{0}\right)$ seja um equilíbrio onde o Jacobiano do sistema em relação as variáveis dinâmicas $(x, y)$ calculado em $\left(x_{0}, y_{0}\right)$ seja nilpotente e não nulo.

Definição 7.0.4. Chamaremos de superfície de candidatos a Bogdanov-Takens o conjunto de pontos $\left(x_{0}, y_{0}, \alpha_{0}, \beta_{0}, \mu_{0}, \delta_{0}, \lambda_{0}\right) \in \mathbb{R}^{+} \times \mathbb{R}^{+} \times \mathcal{W} \times \Delta$ que são candidatos a bifurcação de Bogdanov-Takens.

A partir de agora, vamos nos referir ao Jacobiano do sistema em relação às variáveis dinâmicas $(x, y)$ calculado em um equilíbrio simplesmente como Jacobiano (caso haja con-

fusão quanto ao equilíbrio, o explicitaremos). Caso tratemos do cálculo do Jacobiano de outra aplicação vamos nos referir de forma integral a seu cálculo. 
Na última definição, o Jacobiano ser nilpotente é equivalente a dizer que o determinante e o traço do Jacobiano valem zero. Estas são as condições necessárias para uma bifurcação do tipo Bogdanov-Takens. Restam ainda estudar as condições de transversalidade e de nãodegenerescência.

Na secção um apresentaremos dois lemas que serão usados no restante da análise. Na segunda secção vamos encontrar uma parametrização para a superfície de candidatos a BogdanovTakens. Nas secções três, quatro e cinco, realizaremos o estudo sobre as formas normais das bifurcações de Bogdanov-Takens e suas degenerescências. Na secção seis faremos um estudo algébrico analítico e numérico das condições de não-degenerescência. Na secção sete faremos um estudo algébrico, analítico e numérico das condições de transversalidade. Na secção oito vamos obter informações sobre os centros organizadores (Bogdanov-Takens degenerado, foco-nilpotente e elíptica-nilpotente). Na secção nove, estudaremos quando os parâmetros das bifurcações mais degeneradas são válidos. Por fim, na última secção organizaremos os resultados obtidos no capítulo através de provas formais e aqueles conjecturados com apoio em cálculo numérico.

Ressaltamos que muitos conjuntos que aqui serão utilizados foram definidos no capítulo anterior.

\subsection{Lemas Preliminares}

Vamos enunciar dois lemas que serão usados recorrentemente durante o capítulo.

Lema 7.1.1. Seja $f(z, \delta)=p(z, \delta)+q(z, \delta) \sqrt{h(z, \delta)}$ uma função bem-definida em $I \times J \subseteq \mathbb{R}^{2}$ ( $I$ e J subconjuntos conexos de $\mathbb{R}$ ), na qual:

- $p(z, \delta), q(z, \delta)$ e $h(z, \delta)$ são funções polinomiais em relação a z e $\delta$

- $h(z, \delta)>0$ em $I \times J$

Neste caso, $\left(z_{0}, \delta_{0}\right)$ é uma raiz de $f(z, \delta)$ se, e somente se: 


$$
\begin{aligned}
& \text { i } p\left(z_{0}, \delta_{0}\right)^{2}-q\left(z_{0}, \delta_{0}\right)^{2} h\left(z_{0}, \delta_{0}\right)=0 \\
& \text { ii } p\left(z_{0}, \delta_{0}\right) q\left(z_{0}, \delta_{0}\right)<0 \text { ou } p\left(z_{0}, \delta_{0}\right)=0=q\left(z_{0}, \delta_{0}\right)
\end{aligned}
$$

Demonstração. Imediata.

Durante o estudo, quando estivermos nestas condições, vamos simplesmente nos referir as condições acima como [i] e [ii].

O próximo lema será usado para passar resultados que forem obtidos através de uma mudança de variáveis em $\lambda$ para uma nova variável $z$. Em geral, $K=\left[0, \frac{1}{2}\right]$ e $h(z, \delta)=\frac{z}{x_{i}(z, \delta)}$ onde $i$ será um índice diferente para cada caso.

Lema 7.1.2. Sejam $H=K \times \mathbb{R}^{+}$onde $K$ é compacto, $h: H \mapsto \mathbb{R}^{+}$uma função contínua, $p(z, \delta)$ um polinômio em z e $\delta$ de grau em z no máximo n e no mínimo 1 e $A=\{(z, \delta) \in$ $H ; p(z, \delta)=0\}$. Seja $f: H \mapsto \Delta=\left\{(\delta, \lambda) \in \mathbb{R}^{2} ; \delta>0, \lambda>0\right\}$, onde $f(z, \delta)=(\delta, h(z, \delta))$. Assim, $\Delta / f(A)$ é um conjunto aberto e denso.

Demonstração. Vamos mostrar que $f(A)$ é fechado. Como $A$ é curva algébrica, então ele é um conjunto fechado. Seja $\left(x_{n}\right)=\left(\delta_{n}, \lambda_{n}\right)$ uma sequência em $f(A)$ que converge para $x=(\delta, \lambda)$. Vamos mostrar que $x \in f(A)$. De fato, $\left(\delta_{n}\right)$ obviamente converge para $\delta$. Além disso, como $\lambda_{n}=h\left(z_{n}, \delta_{n}\right)$ e $K$ é compacto, podemos assumir que $z_{n}$ converge para $z$. Dessa forma, temos que $\left(z_{n}, \delta_{n}\right)$ converge para $(z, \delta)$ e, por continuidade de $f, f\left(z_{n}, \delta_{n}\right)$ converge para $f(z, \delta)$. Assim, como o limite é único, $x=f(\delta, z)$ e portanto $f(A)$ é fechado.

Resta mostrar que $\Delta / f(A)$ é denso. Para isso, note que, fixado $\delta=\delta_{0}, p\left(z, \delta_{0}\right)$ tem, no máximo $n$ soluções. Assim, como $f^{-1}\left(\delta_{0}, \lambda\right) \subset K \times\left\{\delta_{0}\right\}$ e existe apenas um número finito de pontos que pertencem a $A$ em $K \times\left\{\delta_{0}\right\}$, então existem apenas um número finito de pontos de $f(A)$ em $\left\{\delta_{0}\right\} \times \mathbb{R}^{+}$. Logo esses pontos são isolados, e $\Delta / f(A)$ é denso. 


\subsection{Estudo da superfície de candidatos a Bogdanov- Takens}

Para começar, vale notar que o Jacobiano calculado em equilíbrios fora dos eixos nunca pode se anular. De fato, $\left(f_{1}\right)_{y}=-\frac{x}{\alpha x^{2}+\beta x+1} \neq 0$ sempre que $x>0$. Assim, os equilíbrios com parâmetros onde o Jacobiano é nilpotente são candidatos a bifurcação Bogdanov-Takens.

Para o Jacobiano ser nilpotente, basta que $W=0$ e $t r=0$ (ver capítulo 6 para definições). Assim, vamos começar achando uma parametrização para os pontos nesta condição:

Lema 7.2.1. Fixado $(\delta, \lambda) \in \Delta$, o conjunto de $\mathcal{F}_{\delta, \lambda}$ no qual $W=0$ e tr $=0$ é uma curva. Essa curva pode ser dividida em dois ramos: $\left(x, y_{B T 1}(x, \delta, \lambda), \beta_{t r}\left(x, y_{B T 1}(x, \delta, \lambda), \delta, \lambda\right), \delta, \lambda\right) e$ $\left(x, y_{B T 2}(x, \delta, \lambda), \beta_{t r}\left(x, y_{B T 1}(x, \delta, \lambda), \delta, \lambda\right), \delta, \lambda\right)$, sendo que, cada um deles, é totalmente parametrizada por $x$. Essa curva não pertence a $\mathcal{F}$ caso $x \lambda \notin] 0,3 / 2 \delta+1 / 2-\sqrt{2} \delta]$ e ela é regular caso $x \lambda \in] 0,3 / 2 \delta+1 / 2-\sqrt{2} \delta\left[\right.$ (ver capítulo 6 para definições de $\mathcal{F}_{\delta, \lambda}$ e $\left.\mathcal{F}\right)$.

Demonstração. Já sabemos que $\operatorname{tr}(x, y, \beta, \delta, \lambda)=0$ se, e somente se, $\beta=\beta_{t r}(x, y, \delta, \lambda)$. Assim, vamos substituir $\beta$ por $\beta_{\text {tr }}$ na equação de $W$ :

$$
\begin{gathered}
W\left(x, y, \beta_{t r}, \delta, \lambda\right)= \\
\left(\delta^{2} y^{2}+\left(3 \lambda x^{2}+3 \lambda x^{2} \delta-2 \lambda^{2} x^{3}-x-3 x \delta\right) y+2 \lambda^{2} x^{4}+2 x^{2}-4 \lambda x^{3}\right)\left(x^{3}\right. \\
\left.(-1+\lambda x)^{2} y^{3}\right)^{-1}
\end{gathered}
$$

O termo que multiplica $y^{2}$ é não nulo. Assim, esta equação é quadrática em y e sempre possui duas soluções. Logo, de $W\left(x, y, \beta_{t r}, \delta, \lambda\right)=0$ obtemos duas soluções em $y$ :

$$
\begin{gathered}
y_{B T 1}=1 / 2 \frac{\left(2 \lambda x-1-3 \delta+\sqrt{4 \lambda^{2} x^{2}-4 \lambda x-12 \lambda x \delta+1+6 \delta+\delta^{2}}\right)(-1+\lambda x) x}{\delta^{2}} \\
y_{B T 2}=-1 / 2 \frac{\left(-2 \lambda x+1+3 \delta+\sqrt{4 \lambda^{2} x^{2}-4 \lambda x-12 \lambda x \delta+1+6 \delta+\delta^{2}}\right)(-1+\lambda x) x}{\delta^{2}}
\end{gathered}
$$

Obtemos dois ramos de solução para o sistema $t r=0$ e $W=0$ :

$$
\begin{aligned}
& \left(x, y_{B T 1}(x, \delta, \lambda), \beta_{t r}\left(x, y_{B T 1}(x, \delta, \lambda), \delta, \lambda\right), \delta, \lambda\right) \\
& \left(x, y_{B T 2}(x, \delta, \lambda), \beta_{t r}\left(x, y_{B T 2}(x, \delta, \lambda), \delta, \lambda\right), \delta, \lambda\right)
\end{aligned}
$$

Para não carregar a notação, vamos nos referir a esses ramos simplesmente como:

$$
\begin{aligned}
& \left(x, y_{B T 1}, \beta_{t r}, \delta, \lambda\right) \\
& \left(x, y_{B T 2}, \beta_{t r}, \delta, \lambda\right)
\end{aligned}
$$


Agora é interessante saber quando que esses ramos são válidos, ou seja, quando que $y_{B T 1} \mathrm{e}$ $y_{B T 2}$ são reais positivos. Tomemos o radicando:

$$
(2 \lambda x-3 \delta-1)^{2}-8 \delta^{2}
$$

Note que, caso este seja positivo, ele é menor do que o quadrado de $2 \lambda x-3 \delta-1$. Assim, caso o radicando seja positivo, para que $y_{B T 1}$ e $y_{B T 2}$ sejam positivos (lembremos que em $\left.\mathcal{F}_{\delta, \lambda}, x<\frac{1}{\lambda}\right)$, devemos ter $2 \lambda x-3 \delta-1<0$, ou seja, devemos ter $x \lambda<3 / 2 \delta+1 / 2$.

Agora, para que $y_{B T 1}$ e $y_{B T 2}$ sejam reais, o radicando deve ser não negativo. Igualando o radicando a zero e resolvendo a equação em função de $x \lambda$ obtemos:

$$
\begin{aligned}
& (x \lambda)_{1}=3 / 2 \delta+1 / 2+\sqrt{2} \delta \\
& (x \lambda)_{2}=3 / 2 \delta+1 / 2-\sqrt{2} \delta
\end{aligned}
$$

Como o termo quadrático em $x \lambda$ do radicando é positivo, temos que, caso $x \lambda \notin](x \lambda)_{2},(x \lambda)_{1}[$, então o radicando é não negativo. No entanto devemos ter $x \lambda<3 / 2 \delta+1 / 2$. Assim sendo:

$$
x \lambda \leq 3 / 2 \delta+1 / 2-\sqrt{2} \delta
$$

para que $y_{B T 1}$ e $y_{B T 2}$ sejam reais e positivos.

Por fim, notemos que, fixado $(\delta, \lambda) \in \Delta$, esses ramos nada mais são do que curvas em $\mathcal{F}_{\delta, \lambda}$. Pelas expressões, sabemos que $y_{B T 1}$ e $y_{B T_{2}}$ são certamente regulares caso $x \lambda<3 / 2 \delta+$ $1 / 2-\sqrt{2} \delta$. Dessa forma, $\beta_{t r}$ calculado em $y_{B T 1}$ e $y_{B T_{2}}$ é regular para $x \lambda<3 / 2 \delta+1 / 2-\sqrt{2} \delta$ e consequentemente a curva é regular em $0<x \lambda<3 / 2 \delta+1 / 2-\sqrt{2} \delta$.

Agora vamos estudar quando estes ramos são válidos.

Lema 7.2.2. Fixado $(\delta, \lambda) \in \Delta$, o ramo $\left(x, y_{B T 2}, \beta_{t r}, \delta, \lambda\right)$ não intersecta $\mathcal{S}$ (ver capítulo 6 para a definição de $\mathcal{S})$.

Demonstração. Calculemos a função $M$ sobre o ramo $\left(x, y_{B T 2}, \beta_{t r}, \delta, \lambda\right)$ :

$$
\begin{gathered}
M\left(x, y_{B T 2}, \beta_{t r}, \delta, \lambda\right)= \\
\frac{2 \delta^{3}\left(-2 \lambda x+1+\delta+\sqrt{4 \lambda^{2} x^{2}-4 \lambda x-12 \lambda x \delta+1+6 \delta+\delta^{2}}\right)}{x(-1+\lambda x)\left(-2 \lambda x+1+3 \delta+\sqrt{4 \lambda^{2} x^{2}-4 \lambda x-12 \lambda x \delta+1+6 \delta+\delta^{2}}\right)^{2}}
\end{gathered}
$$




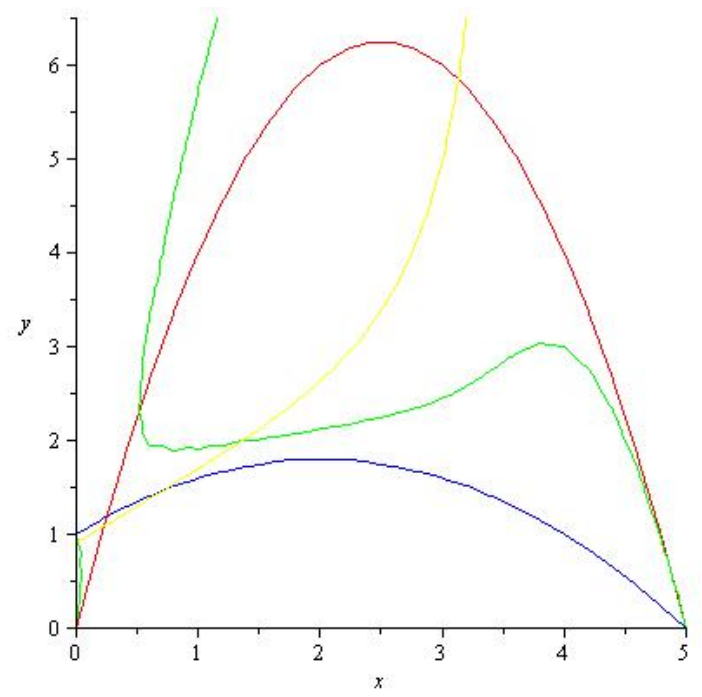

Figura 7.1: Em azul a curva $A=0$, em vermelho $M=0$, em verde $W=0$ e em amarelo $t r=0$. Para o cálculo deste gráfico, tomamos $\delta=0.2, \lambda=0.2$ e $\beta=1$. Note que temos dois cruzamentos da verde com a amarela, apesar de apenas um deles ser para parâmetros válidos

Note que o denominador é negativo em $\mathcal{F}$. Afirmo que o numerador é positivo e portanto $M<0$, o que encerra a demonstração. De fato, como $2 \lambda x<(3-2 \sqrt{2}) \delta+1<\delta+1$, então $2 \lambda x-\delta-1<0$. Isso implica que $-2 \lambda x+\delta+1>0$. Assim, o numerador é soma de termos positivos, multiplicado por um termo positivo. Logo ele é positivo e temos o resultado.

Agora, só precisamos nos preocupar com o primeiro ramo da curva. Comecemos estudando quando que esta curva está contida no conjunto $M \geq 0$ :

Lema 7.2.3. O ramo $\left(x, y_{B T 1}, \beta_{t r}, \delta, \lambda\right)$ está contida em $M \geq 0$ se, e somente se, $\left.\left.x \in\right] 0, \frac{1}{2 \lambda}\right]$.

Demonstração. Calculemos a função $M$ sobre o ramo $\left(x, y_{B T 1}, \beta_{t r}, \delta, \lambda\right)$ :

$$
\begin{gathered}
M\left(x, y_{B T 1}, \beta_{t r}, \delta, \lambda\right)= \\
\frac{-2 \delta^{3}\left(2 \lambda x-1-\delta+\sqrt{4 \lambda^{2} x^{2}-4 \lambda x-12 \lambda x \delta+1+6 \delta+\delta^{2}}\right)}{x(-1+\lambda x)\left(2 \lambda x-1-3 \delta+\sqrt{4 \lambda^{2} x^{2}-4 \lambda x-12 \lambda x \delta+1+6 \delta+\delta^{2}}\right)^{2}}
\end{gathered}
$$

novamente o denominador é negativo. Assim, para ser válido, basta o numerador ser negativo. Para isso $2 \lambda x-1-\delta+\sqrt{4 \lambda^{2} x^{2}-4 \lambda x-12 \lambda x \delta+1+6 \delta+\delta^{2}}>0$. Já sabemos que $2 \lambda x-1-\delta<0$. Dessa forma, o radicando deve ser positivo e ser maior ou igual ao quadrado 
de $2 \lambda x-1-\delta$. Note que o radicando pode ser escrito como:

$$
(2 \lambda x-1-\delta)^{2}-4 \delta(2 \lambda x-1)
$$

Logo, o radicando é maior ou igual ao quadrado de $2 \lambda x-1-\delta$, caso $2 \lambda x-1 \leq 0$, ou seja, $\lambda x \leq \frac{1}{2}$. Caso contrário, $M\left(x, y_{B T 1}, \beta_{t r}, \delta, \lambda\right)<0$.

Resta ainda estudar as restrições com respeito a $A \geq 0$. Com esse objetivo, temos o seguinte lema:

Lema 7.2.4. Existe uma curva $\Gamma_{1}$ em $\Delta$ que divide $\Delta$ em duas componentes conexas: uma acima da curva que chamamos de $\Sigma_{1}$ e uma abaixo da curva que chamamos de $\Sigma_{2}$. Para $(\delta, \lambda) \in \Sigma_{1}$, a função A calculada sobre o ramo $\left(x, y_{B T 1}, \beta_{t r}, \delta, \lambda\right)$ com $0<x \lambda \leq \frac{1}{2}$ é positiva. Já para $(\delta, \lambda) \in \Sigma_{2}$, a função $A$ calculada sobre o ramo $\left(x, y_{B T 1}, \beta_{t r}, \delta, \lambda\right)$ com $0<x \lambda \leq$ $\frac{1}{2}$ se anula duas vezes; dessa forma o ramo $\left(x, y_{B T 1}, \beta_{t r}, \delta, \lambda\right)$ pode ser fatorado em três componentes conexas, duas nas quais $A$ é positivo e uma na qual A é negativo. Sobre a curva $\Gamma_{1}$, o comportamento da função $A$ é igual ao apresentado no conjunto $\Sigma_{1}$.

Para provar este lema, vamos fazer uma mudança de variáveis e interpretar o seu significado. O método usado nesta demonstração será repetido em demonstrações subsequentes e, por isso, vamos dar especial atenção a esta.

Demonstração. Vamos começar calculando a função $A$ sobre o ramo $\left(x, y_{B T 1}, \beta_{t r}, \delta, \lambda\right)$ :

$$
\begin{gathered}
A\left(x, y_{B T 1}, \beta_{t r}, \delta, \lambda\right)= \\
\left(4 \lambda^{2} x^{3}-4 \lambda x^{2}-8 \lambda x^{2} \delta+2 \sqrt{4 \lambda^{2} x^{2}-4 \lambda x-12 \lambda x \delta+1+6 \delta+\delta^{2}} \lambda x^{2}\right. \\
+2 \lambda x \delta^{2}+4 x \delta-x \delta \sqrt{4 \lambda^{2} x^{2}-4 \lambda x-12 \lambda x \delta+1+6 \delta+\delta^{2}}+x \delta^{2}+x \\
\left.-x \sqrt{4 \lambda^{2} x^{2}-4 \lambda x-12 \lambda x \delta+1+6 \delta+\delta^{2}}-2 \delta^{2}\right)\left(2 x^{2}(-1+\lambda x) \delta^{2}\right)^{-1}
\end{gathered}
$$

gostaríamos de fixar $(\delta, \lambda)$ e descobrir para quais $x, A\left(x, y_{B T 1}, \beta_{t r}, \delta, \lambda\right)=0$. Para isso, vamos fazer a seguinte mudança de coordenadas:

$$
z=x \lambda
$$

essa nova variável só pode assumir valores entre zero e meio. Esta variável pode ser interpretada como o domínio da parte válida de $\left(x, y_{B T 1}, \beta_{t r}, \delta, \lambda\right)$ normalizada. Feita essa mudança, 
passamos a ter:

$$
\begin{gathered}
A\left(x, y_{B T 1}, \beta_{t r}, \delta, z\right)= \\
\left(4 z^{2} x+x \delta^{2}+x-4 z x-8 z x \delta+2 \sqrt{4 z^{2}-4 z-12 z \delta+1+6 \delta+\delta^{2}} z x+2 z \delta^{2}+4 x \delta\right. \\
\left.-x \delta \sqrt{4 z^{2}-4 z-12 z \delta+1+6 \delta+\delta^{2}}-x \sqrt{4 z^{2}-4 z-12 z \delta+1+6 \delta+\delta^{2}}-2 \delta^{2}\right) \\
\left(2 x^{2}(-1+z) \delta^{2}\right)^{-1}
\end{gathered}
$$

que não depende mais de $\lambda$. Além disso, o seu denominador não se anula em $\mathcal{F}$ e o numerador é linear em x. Podemos verificar atráves do lema 7.1.1 que o termo que multiplica $x$ não se anula para $\delta>0$ e $0<z<\frac{1}{2}$, de forma que, se $\left.z \in\right] 0, \frac{1}{2}[$, podemos resolver a equação em $x$ sem perder soluções. No entanto, podemos estender esse resultado para $z=\frac{1}{2}$, já que:

$$
A\left(x, y_{B T 1}, \beta_{t r}, \delta, \frac{1}{2}\right)=-\delta^{2} \neq 0
$$

Assim, podemos resolver totalmente a equação $A=0$ em função de x:

$$
\begin{aligned}
x_{A}= & \left(-2 \delta^{2}(-1+z)\right)\left(4 z^{2}+\delta^{2}+1-4 z-8 z \delta+2 \sqrt{4 z^{2}-4 z-12 z \delta+1+6 \delta+\delta^{2}} z\right. \\
& \left.+4 \delta-\delta \sqrt{4 z^{2}-4 z-12 z \delta+1+6 \delta+\delta^{2}}-\sqrt{4 z^{2}-4 z-12 z \delta+1+6 \delta+\delta^{2}}\right)^{-1}
\end{aligned}
$$

Agora, devemos interpretar o que conseguimos. Fixando $\delta>0$ e $z \in] 0, \frac{1}{2}[$, existe um único $x$ tal que $A$ vale zero. Além disso, como $x, z$ e $\lambda$ estão relacionados, sabemos que $\frac{z}{x_{A}}=\lambda$. Assim, fixado $\delta>0$ e $z$, existe um único $x$ e um único $\lambda$ que satisfaz à equação $A=0$. Logo, permitindo que $z$ varie, obtemos uma família a um parâmetro de coordenadas $(x, \lambda)$ que satisfaz à equação $A=0$. Assim, fixando $\lambda$, devemos verificar quais são os valores de $z$ que são levados neste $\lambda$, ou seja, devemos tomar o conjunto $\left(\frac{z}{x_{A}}\right)^{-1}(\lambda)$. Fixado $\lambda$, para cada $z$ em $\left(\frac{z}{x_{A}}\right)^{-1}(\lambda)$, existe um único $x$. Assim, obtemos os pontos $x$ nos quais $A=0$ para $(\delta, \lambda) \in \Delta$ fixo.

Dessa forma, fixando $(\delta, \lambda)$, os parâmetros z que anulam $A$ equivalem à imagem inversa de $\lambda$ pela função $\frac{z}{x_{A}} \operatorname{com} \delta$ fixo. Como $\lambda$ é fixo, é fácil trocar $z$ por $x$, de forma a não utilizar mais esta variável. O caso que escolhemos na figura 7.2 ilustra o caso geral: para cada $\delta$ fixo, existe um $\lambda$ máximo no qual $A$ pode zerar, que chamaremos de $\lambda_{\delta}$. Para $\lambda \geq \lambda_{\delta}$, o ramo $\left(x, y_{B T 1}, \beta_{t r}, \delta, \lambda\right)$ permanece no conjunto $A \geq 0$. Já quando $\lambda<\lambda_{\delta}$, a função $A$ possui dois zeros, sendo que, entre eles, a função $A$ é negativa e portanto o ramo $\left(x, y_{B T 1}, \beta_{t r}, \delta, \lambda\right)$ não está totalmente contido em $\mathcal{S}$. 


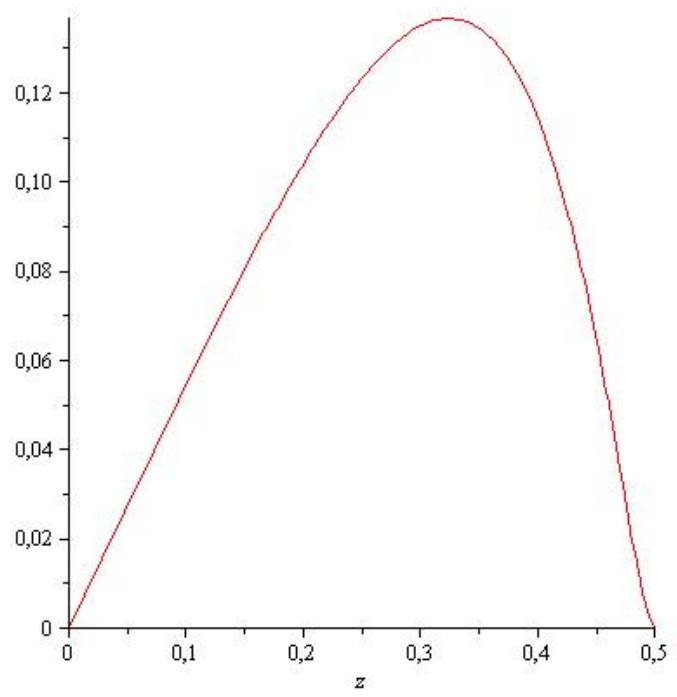

Figura 7.2: Gráfico de $\frac{z}{x_{A}}$. Tal gráfico foi obtido $\operatorname{com} \delta=0.2$

A existência de um $\lambda_{\delta}$ não é difícil de ser provada: de fato, $x_{A}$ não se anula para $z \neq 1$. Dessa forma, a função $\frac{z}{x_{A}}$ é contínua em $\left.z \in\right] 0, \frac{1}{2}$ [. Como em $z=0$ e $z=\frac{1}{2}$ ela possui uma extensão finita $(=0)$, ela é contínua em um compacto e, portanto, possui um máximo que chamamos de $\lambda_{\delta}$. A partir de agora, consideramos essa extensão como a própria função.

Para mostrar que: quando $\lambda<\lambda_{\delta}$ temos exatamente dois zeros, precisamos estudar o comportamento de $\frac{z}{x_{A}}$. Primeiramente, notemos que:

$$
\begin{aligned}
& \frac{z}{x_{A}}(0)=0 \\
& \frac{z}{x_{A}}\left(\frac{1}{4}\right)>0 \\
& \frac{z}{x_{A}}\left(\frac{1}{2}\right)=0
\end{aligned}
$$

Assim, $\frac{z}{x_{A}}$ se anula nos extremos e tem pelo menos um ponto onde ela é positiva. Assim, para estudarmos essa função, vamos começar procurando os zeros de sua derivada em relação a $z$. Mostraremos que, fixada $\delta>0$, a sua derivada se anula em um único ponto $z_{0}$, que, portanto, corresponde ao máximo da função $\left(\frac{z_{0}}{x_{A}\left(z_{0}\right)}=\lambda_{\delta}\right)$. Dessa forma, para $z<z_{0}$ a função é injetora e sua imagem preenche $\left[0, \lambda_{\delta}\left[\mathrm{e}\right.\right.$, para $z>z_{0}$ a função é injetora e sua imagem preenche $\left[0, \lambda_{\delta}\left[\right.\right.$. Logo, para cada $\lambda<\lambda_{\delta}$, existem dois pontos na imagem inversa de $\lambda$. 
Dessa forma, precisamos mostrar que a derivada de $\frac{z}{x_{A}}$ com respeito a $z$ possui um único zero entre ]0, $\frac{1}{2}$ [. Como o denominador de $\frac{z}{x_{A}}$ é diferente de zero, o denominador de sua derivada também é diferente de zero. Assim, o numerador de $\frac{\partial}{\partial z}\left(\frac{z}{x_{A}}\right)$ é igual a:

$$
\frac{\partial}{\partial z}\left(\frac{z}{x_{A}}\right)=p(z, \delta)+q(z, \delta) \sqrt{4 z^{2}-4 z-12 z \delta+1+6 \delta+\delta^{2}}
$$

onde:

$$
\begin{gathered}
p(z, \delta)=-1+10 z+48 z \delta+22 z \delta^{2}-8 z^{2} \delta^{2}-\delta^{3}-7 \delta^{2} \\
-7 \delta-32 z^{2}-88 z^{2} \delta+40 z^{3}-16 z^{4}+40 z^{3} \delta \\
q(z, \delta)=-16 z^{2}-8 z^{2} \delta+8 z-4 \delta+16 z \delta-\delta^{2}-1+8 z^{3}
\end{gathered}
$$

Dessa forma, estamos nas condições do lema 7.1.1. A condição [i] é análoga a:

$$
\begin{aligned}
0= & 4 \delta^{2}(-1+2 z)\left(16 z^{4}+32 z^{4} \delta-112 z^{3} \delta-40 z^{3}+128 z^{2} \delta\right. \\
& \left.+32 z^{2}+4 z^{2} \delta^{2}-10 z-6 z \delta^{2}-48 z \delta+6 \delta+\delta^{2}+1\right)
\end{aligned}
$$

Como $z<\frac{1}{2}$ e $\delta>0$, quando o termo que multiplica $\delta^{2}\left(4 z^{2}-6 z+1\right)$ é diferente de zero, só temos duas possibilidades de solução:

- $\delta=d 1$

- $\delta=d 2$

Agora, $4 z^{2}-6 z+1=0$ com $z<\frac{1}{2}$ se, e somente se, $z=\frac{3}{4}-\frac{\sqrt{5}}{4}$. Ao substituirmos esse resultado na condição [i], obtemos que $\delta=0$ como solução, que pode ser descartada. Logo, as soluções do sistema são apenas $\delta=d 1$ e $\delta=d 2 \operatorname{com} z \neq \frac{3}{4}-\frac{\sqrt{5}}{4}$.

No entanto, ainda resta a restrição [ii] para que seja solução:

$$
p(z, \delta) q(z, \delta)<0
$$

Assim, substituindo em $p(z, \delta) q(z, \delta), \delta$ por $d_{1}$, obtemos que o único zero da função $p(z, \delta) q(z, \delta)$ em $z \in] 0, \frac{1}{2}\left[\right.$ é $1-\frac{\sqrt{2}}{2}$. Da mesma forma, substituindo em $p(z, \delta) q(z, \delta), \delta$ por $d_{2}$, obtemos a mesma coisa. Assim, fazendo uma análise gráfica, obtemos o seguinte:

$$
\text { - } \left.p\left(z, d_{1}\right) q\left(z, d_{1}\right)>0 \text { para } z \in\right] 0, \frac{3}{4}-\frac{\sqrt{5}}{4}[
$$


- $p\left(z, d_{2}\right) q\left(z, d_{2}\right)>0$ para $\left.z \in\right] 0, \frac{3}{4}-\frac{\sqrt{5}}{4}[$

- $p\left(z, d_{1}\right) q\left(z, d_{1}\right) \geq 0$ para $\left.\left.z \in\right] \frac{3}{4}-\frac{\sqrt{5}}{4}, 1-\frac{\sqrt{2}}{2}\right]$

- $p\left(z, d_{1}\right) q\left(z, d_{2}\right)<0$ para $\left.z \in\right] \frac{3}{4}-\frac{\sqrt{5}}{4}, 1-\frac{\sqrt{2}}{2}[$

- $p\left(z, d_{1}\right) q\left(z, d_{1}\right)<0$ para $\left.\left.z \in\right] 1-\frac{\sqrt{2}}{2}, \frac{1}{2}\right]$

- $p\left(z, d_{2}\right) q\left(z, d_{2}\right) \geq 0$ para $z \in\left[1-\frac{\sqrt{2}}{2}, \frac{1}{2}\right]$

Assim, para cada $z \in] \frac{3}{4}-\frac{\sqrt{5}}{4}, \frac{1}{2}\left[\right.$ existe um único $\delta$ que anula $\left(\frac{z}{x_{A}}\right)_{z}$. Além disso, como:

- em $z=\frac{3}{4}-\frac{\sqrt{5}}{4}$, a derivada é um polo

- a expressão da derivada vale zero em $z=\frac{1}{2}$

- a derivada de $d 1$ e $d 2$ pode ser verificada (algebricamente) como diferente de zero em $] \frac{3}{4}-\frac{\sqrt{5}}{4}, 1-\frac{\sqrt{2}}{2}[\cup] 1-\frac{\sqrt{2}}{2}, \frac{1}{2}[$ e numericamente a transição entre $d 1$ e $d 2$ no ponto $z=1-\frac{\sqrt{2}}{2}$ é decrescente.

Assim, temos que, para todo $\delta>0$ fixo, existe um único $z \in] 0, \frac{1}{2}\left[\right.$ no qual $\left(\frac{z}{x_{A}}\right)_{z}$ se anula.

Falta ainda mostrar que existe uma curva contínua em $\Delta$ que divide $\Delta$ em duas componentes conexas, uma na qual o ramo $\left(x, y_{B T 1}, \beta_{t r}, \delta, \lambda\right)$ está totalmente contido em $A \geq 0$ e outra na qual ele possui duas componentes conexas em $A \geq 0$. Parametrizar essa curva não é tão fácil quanto mostrar a sua existência. De fato, para cada $\delta$, existe um único $\lambda_{\delta}$. Tal ponto deve variar continuamente em relação a $\delta$, uma vez que a função $\frac{z}{x_{A}}$ é contínua em $\delta$ e $z \in\left[0, \frac{1}{2}\right]$ que é compacto. Chamemos a curva contínua $\left(\delta, \lambda_{\delta}\right)$ de $\Gamma_{1}$. Tal curva possui um único ponto para cada $\delta$ fixo. Ela, portanto, divide $\Delta$ em duas componentes conexas.

Agora, note que o termo que multiplica $x$ na expressão de $A(x, z, \delta)$ é negativo (faça a devida mudança para o denominador ser positivo). Assim, caso $x>x_{A}$, temos $A(x, \delta, z)<0$. Como $x>x_{A}$ implica $\frac{z}{x}<\frac{z}{x_{A}}$ isso implica que abaixo do gráfico, $A(x, \delta, z)<0$. Analogamente, acima do gráfico, $A(x, \delta, z)>0$. 


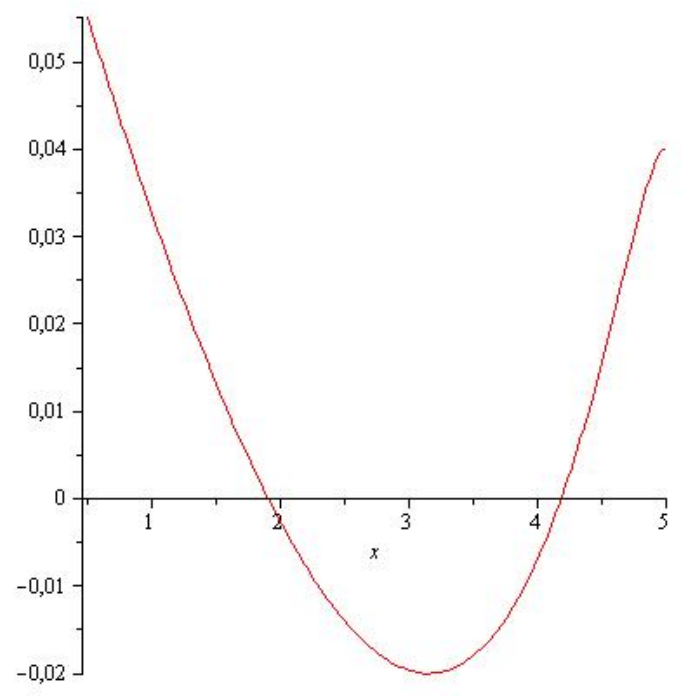

Figura 7.3: Gráfico de $A\left(x, y_{B T 1}, \beta_{t r}, \delta, \lambda\right)$. Para o cálculo deste gráfico, tomamos $\delta=0.2$ e $\lambda=0.1$. Compare com a figura 7.2 .

Desta forma, acima da curva $\Gamma_{1}$, o ramo $\left(x, y_{B T 1}, \beta_{t r}, \delta, \lambda\right)$ está totalmente contido em $A \geq 0$ e abaixo da curva o ramo $\left(x, y_{B T 1}, \beta_{t r}, \delta, \lambda\right)$ possui duas componentes conexas em $A \geq 0$ e uma componente fora.

Antes de continuarmos, podemos tirar um corolário da demonstração do lema anterior:

Corolário 7.2.5. Sobre a curva de candidatos a bifurcação de Bogdanov-Takens, a função A se anula, se e somente se, $x=x_{A}(z, \delta)$, onde $z=x \lambda$. Está função está bem definida para $z \in] 0, \frac{1}{2}\left[\right.$ e qualquer $\delta>0$. Além disso, a função $\frac{z}{x_{A}}$ está bem definida para $z \in\left[0, \frac{1}{2}\right]$ e qualquer $\delta>0$. Fixado $\delta>0$, tal função possui um único ponto onde sua derivada em relação a z é igual a zero, e tal ponto é um máximo. Abaixo deste gráfico $A(x, \delta, z)<0$ e acima deste gráfico $A(x, \delta, z)>0$.

Agora, vamos estudar quando a função $\beta_{t r}\left(x, y_{B T 1}, \delta, \lambda\right)$ é positiva:

Lema 7.2.6. Para todos os pontos de $\left(x, y_{B T 1}, \beta_{t r}, \delta, \lambda\right)$ com $0<x \lambda \leq \frac{1}{2}$, nos quais $A \leq 0$, $\beta_{t r}\left(x, y_{B T 1}, \delta, \lambda\right)>0$.

Demonstração. Vamos proceder da mesma forma que na demonstração anterior. Vamos 
fazer a mudança $x \lambda=z$ em $\beta_{t r}\left(x, y_{B T 1}, \delta, \lambda\right)$ :

$$
\begin{aligned}
& \beta_{t r}\left(x, y_{B T 1}, \delta, z\right)=\left(-6 z^{2} x+11 z x \delta+7 x z-3 \sqrt{4 z^{2}-12 \delta z-4 z+\delta^{2}+6 \delta+1} z x\right. \\
& -4 z \delta^{2}+x \delta \sqrt{4 z^{2}-12 \delta z-4 z+\delta^{2}+6 \delta+1}+2 x \sqrt{4 z^{2}-12 \delta z-4 z+\delta^{2}+6 \delta+1} \\
& \left.-7 x \delta-2 x-x \delta^{2}+4 \delta^{2}\right)\left(2(-1+z) x \delta^{2}\right)^{-1}
\end{aligned}
$$

cujo numerador é linear em relação a $x$. Neste caso, o termo que multiplica $x$ é sempre não nulo e, portanto, é claro que podemos achar os zeros desta função em função de $x$. Assim, resolvendo a equação $\beta_{t r}\left(x, y_{B T 1}, \delta, z\right)=0$ em $x$ obtemos $x_{B}(z, \delta)$, que é sempre maior que zero para $\left.z \in] 0, \frac{1}{2}\right]$ e $\delta>0$. É interessante notar que o termo que multiplica $x$ no numerador de $\beta_{t r}\left(x, y_{B T 1}, \delta, z\right)$ é positivo (faça a devida mudança para que o denominador seja positivo). Assim, caso $x>x_{B}$, então $\beta_{t r}\left(x, y_{B T 1}, \delta, z\right)>0$. Como, nestas condições $\frac{z}{x_{B}}>\frac{z}{x}$, a parte abaixo do gráfico corresponde aos pontos onde $\beta_{t r}\left(x, y_{B T 1}, \delta, z\right)>0$. Agora, vamos comparar $x_{B} \operatorname{com} x_{A}$ :

$$
\begin{aligned}
x_{A}-x_{B} & =2 \delta^{2}(-1+z)\left(2 z^{2}+\sqrt{4 z^{2}-4 z-12 z \delta+1+6 \delta+\delta^{2}} z-5 z \delta\right. \\
-z- & \left.\delta \sqrt{4 z^{2}-4 z-12 z \delta+1+6 \delta+\delta^{2}}+\delta^{2}+\delta\right)(p(z, \delta, \lambda))^{-1}
\end{aligned}
$$

onde $p(z, \delta, \lambda)$ não se anula para $z \in] 0, \frac{1}{2}\left[\right.$, uma vez que os denominadores de $x_{A}$ e $x_{B}$ não se anulam. Como $\delta^{2}(-1+z)$ nunca se anula, a única parte de $x_{A}-x_{B}$ que pode se anular é:

$$
\begin{gathered}
\left(2 z^{2}+\sqrt{4 z^{2}-4 z-12 z \delta+1+6 \delta+\delta^{2}} z-5 z \delta\right. \\
\left.-z-\delta \sqrt{4 z^{2}-4 z-12 z \delta+1+6 \delta+\delta^{2}}+\delta^{2}+\delta\right)
\end{gathered}
$$

no entanto, essa expressão nunca se anula para $\delta>0$ e $0<z<\frac{1}{2}$ (verifique condição [i] e [ii]). Assim sendo, $x_{A}-x_{B} \neq 0$. Como $x_{A}-x_{B}$ é uma função contínua em $\delta$ e $z$, avaliando ela em qualquer ponto nos retornará sempre o mesmo sinal. Fazendo isso, descobrimos que ela é positiva e, portanto, $x_{A}>x_{B}$ qualquer que seja $\delta>0$ e $0<z<\frac{1}{2}$. Isso significa que $\frac{z}{x_{A}}<\frac{z}{x_{B}}$. Isso significa que, fixado $\lambda$, se, em algum momento $A=0$ sobre a curva, então $\beta$ deve ter o mesmo sinal sobre todos os pontos onde $A=0$. Como abaixo do gráfico $\frac{z}{x_{B}}$, $\beta_{t r}\left(x, y_{B T 1}, \delta, \lambda\right)>0$, então concluímos que o sinal nestes pontos deve ser positivo.

Desta demonstração, tiramos um corolário:

Corolário 7.2.7. Sobre a curva de candidatos a bifurcação de Bogdanov-Takens, a função $\beta_{t r}$ se anula, se e somente se, $x=x_{B}(z, \delta)$, onde $z=x \lambda$. Está função está bem definida 


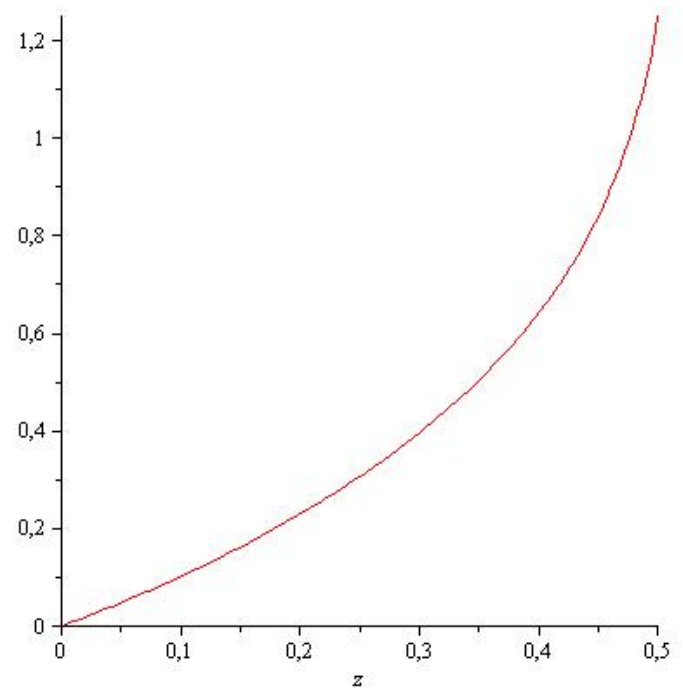

Figura 7.4: Gráfico de $\frac{z}{x_{B}}$. Para o cálculo deste gráfico, tomamos $\delta=0.2$.

para $\left.z \in] 0, \frac{1}{2}\right]$ e qualquer $\delta>0$. Além disso, a função $\frac{z}{x_{B}}$ está bem definida para $z \in\left[0, \frac{1}{2}\right] e$ qualquer $\delta>0$. Abaixo deste gráfico $\beta_{t r}(x, \delta, z)>0$ e acima deste gráfico $\beta_{t r}(x, \delta, z)<0$.

Gostaríamos de provar um resultado para $\beta>0$ semelhante ao do lema sobre $A \geq$ 0. Através da análise gráfica e numérica de $x_{B}$ e $\frac{z}{x_{B}}$, obtemos fortes indícios para uma conjectura:

- Para cada $(\delta, \lambda) \in \Delta$ fixado, existe um único $x$ no qual $\beta_{t r}\left(x, y_{B T 1}, \delta, \lambda\right)=0$.

Para mostrar a conjectura, tentamos tomar a derivada da função $\frac{z}{x_{B}}$ em relação a $z$ e mostrar que ela não possui zeros quando $\delta>0$ e $0<z<\frac{1}{2}$. Não conseguimos provar isso algebricamente, mas temos indícios numéricos de que estejamos neste caso.

Após plotarmos algumas vezes a função $\beta_{t r} \operatorname{com}(\delta, \lambda)$ fixados, como na figura 7.5 , temos a impressão de que essa função é sempre injetora. No entanto, podemos mostrar que isso não é verdade, como vemos na figura 7.6. Desta forma, ao fixarmos $(\delta, \lambda) \in \Delta$ e $\beta>0$, pode existir mais de um candidato a bifurcação do tipo Bogdanov-Takens em $\mathcal{S}_{\beta}$.

Por fim, o principal resultado desta secção pode ser resumido no seguinte teorema: 


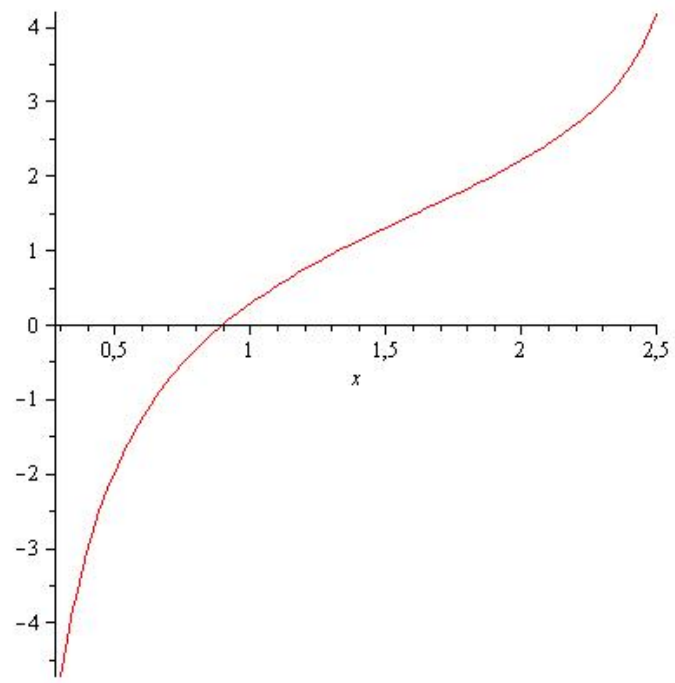

Figura 7.5: Gráfico de $\beta_{t r}\left(x, y_{B T_{1}}(x, \delta, \lambda), \delta, \lambda\right)$. Para o cálculo deste gráfico, tomamos $\delta=0.2 \mathrm{e}$ $\lambda=0.2$. Compare com a figura 7.4.

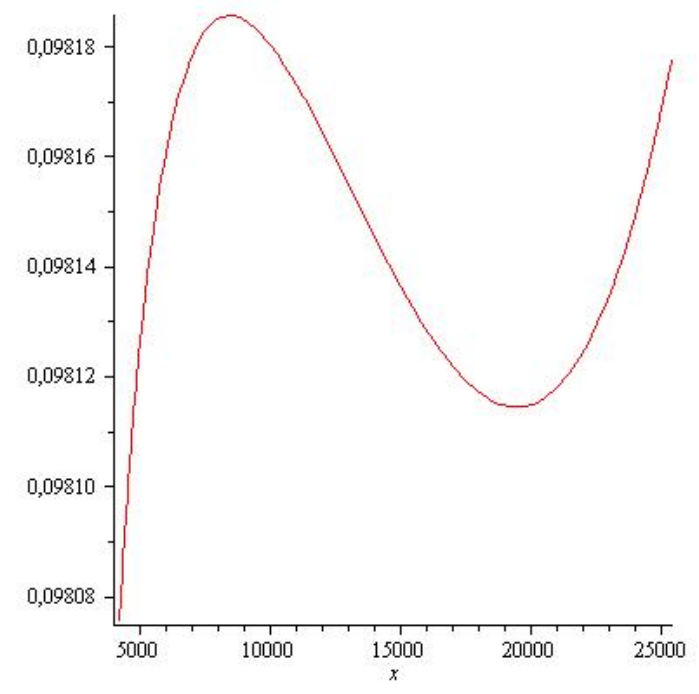

Figura 7.6: Gráfico de $\beta_{t r}\left(x, y_{B T_{1}}(x, \delta, \lambda), \delta, \lambda\right)$. Para o cálculo deste gráfico, tomamos $\delta=10 \mathrm{e}$ $\lambda=\frac{1}{84688.54173}$. Note $x$ é válido até 42344.27086 , e portanto estamos com $x$ válido. A função não é injetora. 
Teorema 7.2.8. Fixado $(\delta, \lambda) \in \Delta$, para todo $0<x \leq \frac{1}{2 \lambda}$ existe um único $y=y_{B T 1}(x, \delta, \lambda)$, um único $\beta=\beta_{t r}\left(x, y_{B T 1}, \delta, \lambda\right)$, um único $\alpha=A\left(x, y_{B T 1}, \beta_{t r}, \delta, \lambda\right)$ e um único $\mu=M\left(x, y_{B T 1}, \beta_{t r}, \delta, \lambda\right)$ tais que o ponto $\left(x, y_{B T 1}\right)$ com os parâmetros $\left(\alpha=A, \beta=\beta_{t r}, \mu=M, \delta, \lambda\right)$ é um equilíbrio no qual o jacobiano do sistema é nilpotente. Nestes pontos, $M \geq 0$. Em nenhum outro ponto $(x, y, \alpha, \beta, \mu, \delta, \lambda),(x, y)$ será um equilíbrio onde o jacobiano é nilpotente e os parâmetros são válidos.

Note que não garantimos que os parâmetros sejam válidos (ver os lemas anteriores) e que a bifurcação seja de fato uma Bogdanov-Takens. Abordamos este assunto a seguir.

\subsection{Forma Normal preliminar}

Agora que já temos uma parametrização para a superfície de candidatos a Bogdanov-Takens precisamos fazer as devidas alterações no sistema dinâmico para comprovar que esses pontos de fato são bifurcações Bogdanov-Takens.

Para isso vamos tratar o caso genericamente, considere que $\left(x_{0}, y_{0}, \alpha_{0}, \beta_{0}, \mu_{0}, \delta_{0}, \lambda_{0}\right)$ seja um candidato a bifurcação de Bogdanov-Takens. Vamos começar localizando o sistema com a mudança de coordenadas:

$$
\begin{aligned}
& x=u+x_{0} \\
& y=v+y_{0}
\end{aligned}
$$

Passamos a ter:

$$
\left\{\begin{array}{c}
\dot{u}=\left(u+x_{0}\right)\left(1-\lambda\left(u+x_{0}\right)\right)-\frac{\left(v+y_{0}\right)\left(u+x_{0}\right)}{\alpha\left(u+x_{0}\right)^{2}+\beta\left(u+x_{0}\right)+1}=g_{1}(u, v, \alpha, \beta, \mu, \delta, \lambda) \\
\dot{v}=\left(v+y_{0}\right)\left(-\delta-\mu\left(v+y_{0}\right)\right)+\frac{\left(v+y_{0}\right)\left(u+x_{0}\right)}{\alpha\left(u+x_{0}\right)^{2}+\beta\left(u+x_{0}\right)+1}=g_{2}(u, v, \alpha, \beta, \mu, \delta, \lambda)
\end{array}\right.
$$

onde $g_{1}\left(0,0, \alpha_{0}, \beta_{0}, \mu_{0}, \delta_{0}, \lambda_{0}\right)=0=g_{2}\left(0,0, \alpha_{0}, \beta_{0}, \mu_{0}, \delta_{0}, \lambda_{0}\right)$. Antes de continuarmos, note que a derivada de $g_{1}$ com respeito a $v$ calculado na origem vale:

$$
-\frac{x_{0}}{\alpha x_{0}^{2}+\beta x_{0}+1}
$$

que é sempre diferente de zero, para todo $\left(x_{0}, y_{0}\right)$ fora dos eixos. 
Agora, tomemos a seguinte mudança de coordenadas:

$$
\begin{gathered}
x=u \\
y=g_{1}(u, v, \alpha, \beta, \mu, \delta, \lambda)
\end{gathered}
$$

Pelo cálculo anterior, esta mudança de coordenadas é um difeomorfismo em uma vizinhança de $\left(0,0, \alpha_{0}, \beta_{0}, \mu_{0}, \delta_{0}, \lambda_{0}\right)$ e portanto é válida. Assim, o sistema 7.1 passa a ser:

$$
\left\{\begin{array}{c}
\dot{x}=y \\
\dot{y}=a 00+a 10 x+a 01 y+a 20 x^{2}+a 11 x y+a 02 y^{2}+\mathcal{O}\left(|(x, y)|^{3}\right)
\end{array}\right.
$$

onde $a 00, a 10, a 01, a 20, a 11$ e $a 02$ dependem de $(\alpha, \beta, \mu, \delta, \lambda)$ e $a 00\left(\alpha_{0}, \beta_{0}, \mu_{0}, \delta_{0}, \lambda_{0}\right)=0$, $a 10\left(\alpha_{0}, \beta_{0}, \mu_{0}, \delta_{0}, \lambda_{0}\right)=0$ e $a 01\left(\alpha_{0}, \beta_{0}, \mu_{0}, \delta_{0}, \lambda_{0}\right)=0$ por hipótese.

Agora, fazendo a mudança de coordenadas:

$$
\begin{gathered}
x=X+\frac{a 02}{2} X^{2} \\
y=Y+a 02 X Y
\end{gathered}
$$

obtemos o sistema:

$$
\left\{\begin{array}{c}
\dot{X}=Y \\
\dot{Y}=a 00+(a 10-a 00 a 02) X+a 01 Y+ \\
+(1 / 2 a 10 a 02+a 20+(-a 10+a 00 \text { a02 }) a 02) X^{2}+a 11 X Y+\mathcal{O}\left(|(X, Y)|^{3}\right)
\end{array}\right.
$$

Assim, quando tivermos $(\alpha, \beta, \mu, \delta, \lambda)=\left(\alpha_{0}, \beta_{0}, \mu_{0}, \delta_{0}, \lambda_{0}\right)$, o sistema passará a ser:

$$
\left\{\begin{array}{c}
\dot{X}=Y \\
\dot{Y}=a 20\left(\alpha_{0}, \beta_{0}, \mu_{0}, \delta_{0}, \lambda_{0}\right) X^{2}+a 11\left(\alpha_{0}, \beta_{0}, \mu_{0}, \delta_{0}, \lambda_{0}\right) X Y+\mathcal{O}\left(|(X, Y)|^{3}\right)
\end{array}\right.
$$

Assim, nos parâmetros $\left(\alpha_{0}, \beta_{0}, \mu_{0}, \delta_{0}, \lambda_{0}\right)$ o sistema 7.1 é $C^{\infty}$-equivalente ao sistema 7.4. Este último, quando $a 11 \neq 0 \neq a 20$, está na forma normal de uma bifurcação do tipo BogdanovTakens. Reunimos este resultado na seguinte proposição:

Proposição 7.3.1. Caso $\left(x_{0}, y_{0}\right)$ com $\left(\alpha_{0}, \beta_{0}, \mu_{0}, \delta_{0}, \lambda_{0}\right)$ seja um equilibrio com jacobiano nilpotente, $a 11\left(x_{0}, y_{0}, \alpha_{0}, \beta_{0}, \mu_{0}, \delta_{0}, \lambda_{0}\right) \neq 0$ e a $20\left(x_{0}, y_{0}, \alpha_{0}, \beta_{0}, \mu_{0}, \delta_{0}, \lambda_{0}\right) \neq 0$ então, o sistema localizado neste equilíbrio é equivalente a:

$$
\left\{\begin{array}{lcc}
\dot{x} & = & y \\
\dot{y} & = & a 20 x^{2}+a 11 x y+\mathcal{O}\left(|(x, y)|^{3}\right)
\end{array}\right.
$$




\subsection{Estudo da forma normal quando $a 11\left(\alpha_{0}, \beta_{0}, \mu_{0}, \delta_{0}, \lambda_{0}\right)$ é igual a zero mas $a 20\left(\alpha_{0}, \beta_{0}, \mu_{0}, \delta_{0}, \lambda_{0}\right)$ é diferente de zero}

Já mostramos que sobre um candidato a bifurcação de Bogdanov-Takens o sistema 1.1 é equivalente ao sistema 7.4. Agora, queremos estudar casos mais degenerados. Vamos começar com o a bifurcação de Bogdanov-Takens de codimensão três. Para esta bifurcação ocorrer devemos ter $a 11\left(\alpha_{0}, \beta_{0}, \mu_{0}, \delta_{0}, \lambda_{0}\right)=0$ mas $a 20\left(\alpha_{0}, \beta_{0}, \mu_{0}, \delta_{0}, \lambda_{0}\right) \neq 0$.

Até agora, o sistema estava na forma:

$$
\left\{\begin{array}{cc}
\dot{x}= & y \\
\dot{y}= & b 20 x^{2}+b 30 x^{3}+b 21 x^{2} y+b 12 x y^{2}+b 03 y^{3}+ \\
& +b 40 x^{4}+b 31 x^{3} y+b 22 x^{2} y^{2}+b 13 x y^{3}+b 04 y^{4}+\mathcal{O}\left(|(x, y)|^{5}\right)
\end{array}\right.
$$

onde $b 20=a 20\left(\alpha_{0}, \beta_{0}, \mu_{0}, \delta_{0}, \lambda_{0}\right) \neq 0$. Agora, vamos fazer a seguinte mudança de coordenadas:

$$
\begin{gathered}
x=\begin{array}{c}
X+1 / 3 \frac{b 21 X Y}{b 20}+1 / 6 b 12 X^{3}+1 / 2 b 03 X^{2} Y+\frac{\left(1 / 12 b 22 b 20+\frac{5}{54} b 21^{2}\right) X^{4}}{b 20}+ \\
\frac{(1 / 6 b 13 b 20+1 / 6 b 12 b 21) X^{3} Y}{b 20}+\frac{(1 / 2 b 21 b 03+1 / 2 b 04 b 20) X^{2} Y^{2}}{b 20}
\end{array} \\
y=Y+1 / 3 \frac{b 21 Y^{2}}{b 20}+1 / 3 b 21 X^{3}+1 / 2 b 12 X^{2} Y+b 03 X Y^{2}+\frac{\left(1 / 3 b 30 b 21+1 / 2 b 03 b 20^{2}\right) X^{4}}{b 20}+ \\
\frac{\left(1 / 3 b 22 b 20+\frac{10}{27} b 21^{2}\right) X^{3} Y}{b 20}+\frac{(1 / 2 b 13 b 20+1 / 2 b 12 b 21) X^{2} Y^{2}}{b 20}+\frac{(b 21 b 03+b 04 b 20) X Y^{3}}{b 20}
\end{gathered}
$$

Desta forma, o sistema passa a ser igual a:

$$
\left\{\begin{array}{cc}
\dot{X}= & Y+\mathcal{O}\left(|(X, Y)|^{5}\right) \\
\dot{Y}= & \text { b20 } X^{2}+b 30 X^{3}-\frac{\left(1 / 6 b 12 b 20^{2}-b 20 b 40\right) X^{4}}{b 20} \\
& -\frac{\left(3 b 03 b 20^{2}-b 31 b 20+b 30 b 21\right) Y X^{3}}{b 20}+\mathcal{O}\left(|(X, Y)|^{5}\right)
\end{array}\right.
$$

Agora, lembremos uma proposição conhecida nestas análises:

Proposição 7.4.1. O sistema:

$$
\left\{\begin{array}{lc}
\dot{x}= & y+\mathcal{O}\left(|(x, y)|^{5}\right) \\
\dot{y}= & x^{2}+c 30 x^{3}+c 40 x^{4}+c 31 x^{3} Y+\mathcal{O}\left(|(x, y)|^{5}\right)
\end{array}\right.
$$


é equivalente a:

$$
\left\{\begin{array}{lc}
\dot{x}= & y+\mathcal{O}\left(|(x, y)|^{5}\right) \\
\dot{y}= & x^{2}+c 31 x^{3} Y+\mathcal{O}\left(|(x, y)|^{5}\right)
\end{array}\right.
$$

Este lema foi inspirado em [?].

Demonstração. De fato, defina $k(x)=x^{2}+c 30 x^{3}+c 40 x^{4}$. Agora, seja $K(x)=\int_{0}^{x} k(s) d s$ e tome:

$$
\begin{gathered}
X=(3 K(x))^{\frac{1}{3}} \\
Y=y
\end{gathered}
$$

Assim, o sistema passa a ser igual a:

$$
\left\{\begin{array}{c}
\dot{X}=(3 K(x))^{\frac{-2}{3}} k(x) Y+\mathcal{O}\left(|(x, Y)|^{5}\right) \\
\dot{Y}=k(x)+c 31 x^{3} Y+\mathcal{O}\left(|(x, Y)|^{5}\right)
\end{array}\right.
$$

Como $(3 K(x))^{\frac{-2}{3}} k(x)=1+\mathcal{O}(x)$, este é um fator que é estritamente positivo em uma vizinhança da origem. Assim, caso tomemos $s=(3 K(x))^{\frac{-2}{3}} k(x) t$ como o novo tempo do sistema, o retrato de fase não vai sofrer alterações qualitativas. Assim sendo, teremos:

$$
\left\{\begin{array}{c}
\dot{X}=Y+\mathcal{O}\left(|(x, Y)|^{5}\right) \\
\dot{Y}=\left(k(x)+c 31 x^{3} Y\right)\left((3 K(x))^{\frac{-2}{3}} k(x)\right)^{-1}+\mathcal{O}\left(|(x, Y)|^{5}\right)
\end{array}\right.
$$

Agora,

$$
\frac{k(x)}{(3 K(x))^{\frac{-2}{3}} k(x)}=\left((3 K(x))^{\frac{1}{3}}\right)^{2}=X^{2}
$$

e

$$
\frac{c 31 x^{3}}{(3 K(x))^{\frac{-2}{3}} k(x)}=\frac{c 31 x^{3} X^{2}}{k(x)}=c 31 X^{2}\left(x+\mathcal{O}\left(x^{2}\right)\right)=c 31 X^{2}\left(X+\mathcal{O}\left(X^{2}\right)\right)
$$

Assim, temos o resultado.

Assim sendo, com uma simples mudança de coordenadas $\left(x=\frac{1}{b 20} X, y=\frac{1}{b 20} Y\right)$ e aplicando a proposição, o sistema passa a ser equivalente a:

$$
\left\{\begin{array}{lc}
\dot{x} & = \\
\dot{y} & =x^{2}-\frac{\left(3 b 03 b 20^{2}-b 31 b 20+b 30 b 21\right) y x^{3}}{b 20^{4}}+\mathcal{O}\left(|(x, y)|^{5}\right)
\end{array}\right.
$$


que está na forma normal relativa a uma bifurcação de Bogdanov-Takens de codimensão três. Para que esta bifurcação ocorra, devemos ter o termo que multiplica $x^{3} y$ diferente de zero. Ou seja:

$$
\begin{gathered}
0 \neq E=3 \text { b03 b20 }{ }^{2}-b 31 \text { b20 }+ \text { b30 b21 }= \\
=\left(\text { a21 a02 a20 - a30 a21 }-3 \text { a03 a20 }{ }^{2}\right)\left(\alpha_{0}, \beta_{0}, \mu_{0}, \delta_{0}, \lambda_{0}\right)
\end{gathered}
$$

onde $E$ é o numerador do coeficiente que multiplica $x^{3} y$.

Assim, o principal resultado da secção pode ser resumida na seguinte proposição:

Proposição 7.4.2. Caso $\left(x_{0}, y_{0}\right)$ com $\left(\alpha_{0}, \beta_{0}, \mu_{0}, \delta_{0}, \lambda_{0}\right)$ seja um equilíbrio com jacobiano nilpotente, $a 11\left(x_{0}, y_{0}, \alpha_{0}, \beta_{0}, \mu_{0}, \delta_{0}, \lambda_{0}\right)=0, a 20\left(x_{0}, y_{0}, \alpha_{0}, \beta_{0}, \mu_{0}, \delta_{0}, \lambda_{0}\right) \neq 0$ e $E\left(x_{0}, y_{0}, \alpha_{0}, \beta_{0}, \mu_{0}, \delta_{0}, \lambda_{0}\right) \neq$ 0 então, o sistema localizado neste equilíbrio é equivalente a:

$$
\begin{cases}\dot{x}= & y+\mathcal{O}\left(|(x, y)|^{5}\right) \\ \dot{y}= & x^{2}+F x^{3} y+\mathcal{O}\left(|(x, y)|^{5}\right)\end{cases}
$$

\subsection{Estudo da forma normal quando $a 20\left(\alpha_{0}, \beta_{0}, \mu_{0}, \delta_{0}, \lambda_{0}\right)$ é igual a zero mas $a 11\left(\alpha_{0}, \beta_{0}, \mu_{0}, \delta_{0}, \lambda_{0}\right)$ é diferente de zero}

Vamos continuar o estudo sobre as bifurcações nilpotentes mais degeneradas do que as de Bogdanov-Takens. Para estes tipos de bifurcação ocorrer devemos ter $a 20\left(\alpha_{0}, \beta_{0}, \mu_{0}, \delta_{0}, \lambda_{0}\right)=$ 0 mas $a 11\left(\alpha_{0}, \beta_{0}, \mu_{0}, \delta_{0}, \lambda_{0}\right) \neq 0$.

Até agora, o sistema era da forma:

$$
\left\{\begin{array}{cc}
\dot{x}= & y \\
\dot{y}= & b 11 x y+b 30 x^{3}+b 21 x^{2} y+b 12 x y^{2}+b 03 y^{3}+ \\
& +b 40 x^{4}+b 31 x^{3} y+b 22 x^{2} y^{2}+b 13 x y^{3}+b 04 y^{4}+\mathcal{O}\left(|(x, y)|^{5}\right)
\end{array}\right.
$$


onde $b 11=a 11\left(\alpha_{0}, \beta_{0}, \mu_{0}, \delta_{0}, \lambda_{0}\right) \neq 0$. Agora, vamos fazer a seguinte mudança de coordenadas:

$$
\begin{gathered}
x=X+1 / 6 b 12 X^{3}+1 / 2 b 03 X^{2} Y+1 / 12 X^{4} b 22-1 / 6 X^{4} b 11 b 03 \\
+1 / 6 b 13 X^{3} Y+1 / 2 b 04 X^{2} Y^{2} \\
y=\quad Y+1 / 2 b 12 X^{2} Y+b 03 X Y^{2}-1 / 6 X^{3} Y b 11 b 03 \\
+1 / 3 X^{3} Y b 22+1 / 2 b 13 X^{2} Y^{2}+b 04 X Y^{3}
\end{gathered}
$$

Desta forma, o sistema passa a ser igual a:

$$
\left\{\begin{array}{cc}
\dot{X}= & Y+\mathcal{O}\left(|(X, Y)|^{5}\right) \\
\dot{Y}= & b 11 X Y+b 30 X^{3}+b 21 X^{2} Y+b 40 X^{4}+ \\
& (1 / 6 b 12 b 11+b 31) Y X^{3}+\mathcal{O}\left(|(X, Y)|^{5}\right)
\end{array}\right.
$$

Aqui já temos a primeira condição de não-degenerescência: devemos ter $b 30 \neq 0$. Definiremos $F 1=b 30$. Dessa forma, temos:

$$
F 1=b 30=a 30
$$

Vamos nos permitir assumir $F 1<0$ (será o caso do sistema trabalhado). Assim, podemos continuar a conta:

Proposição 7.5.1. O sistema:

$$
\left\{\begin{array}{c}
\dot{x}=y+\mathcal{O}\left(|(x, y)|^{5}\right) \\
\dot{y}=c 11 x y-x^{3}+c 21 x^{2} y+c 40 x^{4}+c 31 x^{3} y+\mathcal{O}\left(|(x, y)|^{5}\right)
\end{array}\right.
$$

é equivalente ao sistema:

$$
\left\{\begin{array}{c}
\dot{x}=y+\mathcal{O}\left(|(x, y)|^{5}\right) \\
\dot{y}=d 11 x y-x^{3}+d 21 x^{2} y+d 31 x^{3} y+\mathcal{O}\left(|(x, y)|^{5}\right)
\end{array}\right.
$$

onde $d 11=c 11$ e d21 $=c 21-\frac{3}{5} c 40 c 11$.

Demonstração. Comecemos definindo $k(x)=-x^{3}+c 40 x^{4}$. Agora, seja $K(x)=\int_{0}^{x} k(s) d s$ e tome:

$$
\begin{gathered}
X=(-4 K(x))^{\frac{1}{4}} \\
Y=y
\end{gathered}
$$


Assim, o sistema passa a ser igual a:

$$
\left\{\begin{array}{c}
\dot{X}=(-4 K(x))^{-\frac{3}{4}}(-k(x)) Y+\mathcal{O}\left(|(x, Y)|^{5}\right) \\
\dot{Y}=k(x)+Y\left(c 11 x+c 21 x^{2}+c 31 x^{3}\right)+\mathcal{O}\left(|(x, Y)|^{5}\right)
\end{array}\right.
$$

Como $(-4 K(x))^{-\frac{3}{4}}(-k(x))=1+\mathcal{O}(x)$, este é um fator que é estritamente positivo em uma vizinhança da origem. Assim, caso tomemos $s=(-4 K(x))^{-\frac{3}{4}}(-k(x)) t$ como o novo tempo do sistema, o retrato de fase não vai sofrer alterações qualitativas. Assim sendo, teremos:

$$
\left\{\begin{array}{c}
\dot{X}=Y+\mathcal{O}\left(|(x, Y)|^{5}\right) \\
\dot{Y}=\frac{k(x)}{(-4 K(x))^{-\frac{3}{4}}(-k(x))}+Y \frac{c 11 x+c 21 x^{2}+c 31 x^{3}}{(-4 K(x))^{-\frac{3}{4}}(-k(x))}+\mathcal{O}\left(|(x, Y)|^{5}\right)
\end{array}\right.
$$

Agora, note que:

$$
\frac{k(x)}{(-4 K(x))^{-\frac{3}{4}}(-k(x))}=-\left((-4 K(x))^{-\frac{1}{4}}\right)^{3}=-X^{3}
$$

Procederemos com os cálculos para os coeficientes que multiplicam $X Y$ e $X^{2} Y$. Além destes coeficientes, não precisamos calcular os coeficientes de grau maior, pois não haverá alterações na análise da bifurcação. É interessante notar, no entanto, que não poderão aparecer coeficientes que multipliquem termos de ordem quarta, a não ser que multiplique $X^{3} Y$. Isso é fácil de ver pois $\frac{c 11 x+c 21 x^{2}+c 31 x^{3}}{(-4 K(x))^{-\frac{3}{4}}(-k(x))}$ poderá ser escrito como uma série em $X$.

Assim, temos que:

$$
\frac{c 11 x+c 21 x^{2}+c 31 x^{3}}{(-4 K(x))^{-\frac{3}{4}}(-k(x))}=-X^{3}\left(\frac{c 11 x}{k(x)}+\frac{c 21 x^{2}}{k(x)}+\mathcal{O}\left(x^{0}\right)\right)
$$

Expandindo em série, obtemos que:

$$
\begin{gathered}
\frac{c 11 x}{k(x)}=c 11\left(-\frac{1}{x^{2}}-\frac{c 40}{x}+\mathcal{O}\left(x^{0}\right)\right) \\
\frac{c 21 x^{2}}{k(x)}=c 21\left(-\frac{1}{x}+\mathcal{O}\left(x^{0}\right)\right)
\end{gathered}
$$

Assim, obtemos:

$$
\frac{c 11 x+c 21 x^{2}+c 31 x^{3}}{(-4 K(x))^{-\frac{3}{4}}(-k(x))}=-X^{3}\left(c 11\left(-\frac{1}{x^{2}}-\frac{c 40}{x}\right)+c 21\left(-\frac{1}{x}\right)+\mathcal{O}\left(x^{0}\right)\right)
$$

Assim, precisamos calcular quanto vale $\frac{1}{x^{2}}$ e $\frac{1}{x}$ em função de $X$. Utilizando a equação implícita $X-(-4 K(x))^{-\frac{1}{4}}=0$, podemos calcular os primeiros termos da expansão em série 
de $x(X)$, onde $(-4 K(x(X)))^{-\frac{1}{4}}=X$. Assim, obtemos que:

$$
\begin{gathered}
\frac{1}{x}=\frac{1}{X}+\mathcal{O}\left(X^{0}\right) \\
\frac{1}{x^{2}}=\frac{1}{X^{2}}-\frac{2}{5} c 40 \\
X
\end{gathered}
$$

Substituindo, enfim obtemos:

$$
\frac{c 11 x+c 21 x^{2}+c 31 x^{3}}{(-4 K(x))^{-\frac{3}{4}}(-k(x))}=-X^{3}\left(\frac{-c 11}{X^{2}}+\frac{\frac{3}{5} c 40 c 11-c 21}{X}++\mathcal{O}\left(x^{0}\right)\right)
$$

Assim, temos que o sistema passa a ser igual a:

$$
\left\{\begin{array}{c}
\dot{X}=Y+\mathcal{O}\left(|(X, Y)|^{5}\right) \\
\dot{Y}=-X^{3}+Y\left(c 11 X+\left(c 21-\frac{3}{5} c 40 c 11\right) X^{2}+f 3 X^{3}\right)+\mathcal{O}\left(|(X, Y)|^{5}\right)
\end{array}\right.
$$

como queríamos demonstrar.

Fazendo uso da mudança de coordenadas $x=\sqrt{\|c 30\|} X$ e $y=\sqrt{\|c 30\|} Y$ e assumindo que o $c 30\left(\alpha_{0}, \beta_{0}, \mu_{0}, \delta_{0}, \lambda_{0}\right)<0$ podemos aplicar a proposição. Dessa forma, mostramos que o sistema 7.7 é equivalente a:

$$
\left\{\begin{array}{c}
\dot{x}=y+\mathcal{O}\left(|(x, y)|^{5}\right) \\
\dot{y}=-x^{3}+\frac{b 11}{\sqrt{\|b 30\|}} x y+\frac{\left(b 21\|b 30\|-\frac{3}{5} b 40 b 11\right)}{b 30^{2}} x^{2} y+f 3 x^{3} y+\mathcal{O}\left(|(x, y)|^{5}\right)
\end{array}\right.
$$

Assim, para que a bifurcação seja de codimensão 3 e não seja ainda mais degenerada, devemos ter $\frac{\left(b 21\|b 30\|-\frac{3}{5} b 40 b 11\right)}{b 30^{2}} \neq 0$. Assim, como já assumimos que $b 30 \neq 0$, devemos ter $(b 21\|b 30\|-$ $\left.\frac{3}{5} b 40 b 11\right) \neq 0$. A este termo chamaremos de $F 2$ :

$$
F 2=b 21\|b 30\|-\frac{3}{5} b 40 b 11
$$

Por fim, chamaremos de $F 3$ o modulo do coeficiente que multiplica $x y$ :

$$
F 3=\left\|\frac{b 11}{\sqrt{\|b 30\|}}\right\|
$$

Dependendo do valor de $F 3$ teremos uma bifurcação diferente e um retrato de fase diferente. Além disso $F 3 \neq 2 \sqrt{2}$ para que a bifurcação não seja mais degenerada (ver a referência [10]). Assim, concluímos que, caso $F 1<0, F 2 \neq 0$ e $F 3 \neq 2 \sqrt{2}$, a bifurcação poderá ser de um dos seguintes tipos (ver a referência [10] e o capítulo 10 para maiores detalhes): 
- foco nilpotente

- elíptica nilpotente

Até agora o sistema 7.7 é equivalente a:

$$
\left\{\begin{array}{c}
\dot{x}=y+\mathcal{O}\left(|(x, y)|^{5}\right) \\
\dot{y}=c 11 x y-x^{3}+c 21 x^{2} y+c 31 x^{3} y+\mathcal{O}\left(|(x, y)|^{5}\right)
\end{array}\right.
$$

Vamos fazer uma última simplificação. Assumindo que $b 11=c 11<0$ e $c 21<0$, vamos tomar:

e obtemos:

$$
\begin{gathered}
X=-\|c 21\| x \\
Y=-c 21^{2} y \\
s=\frac{1}{\|c 21\|} t
\end{gathered}
$$

$$
\left\{\begin{array}{c}
\dot{x}=y+\mathcal{O}\left(|(x, y)|^{5}\right) \\
\dot{y}=d 11 x y-x^{3}-x^{2} y+f x^{3} y+\mathcal{O}\left(|(x, y)|^{5}\right)
\end{array}\right.
$$

onde $d 11$ é igual a $F 3$ e, portanto, é positivo.

Assim, podemos resumir o principal resultado da secção na seguinte proposição:

Proposição 7.5.2. Caso $\left(x_{0}, y_{0}\right)$ com $\left(\alpha_{0}, \beta_{0}, \mu_{0}, \delta_{0}, \lambda_{0}\right)$ seja um equilíbrio com jacobiano nilpotente, $a 20\left(x_{0}, y_{0}, \alpha_{0}, \beta_{0}, \mu_{0}, \delta_{0}, \lambda_{0}\right)=0$ e:

- $F 1\left(x_{0}, y_{0}, \alpha_{0}, \beta_{0}, \mu_{0}, \delta_{0}, \lambda_{0}\right)<0$

- $b 11\left(x_{0}, y_{0}, \alpha_{0}, \beta_{0}, \mu_{0}, \delta_{0}, \lambda_{0}\right)<0$

- $F 2\left(x_{0}, y_{0}, \alpha_{0}, \beta_{0}, \mu_{0}, \delta_{0}, \lambda_{0}\right)<0$

- $F 3\left(x_{0}, y_{0}, \alpha_{0}, \beta_{0}, \mu_{0}, \delta_{0}, \lambda_{0}\right) \neq 2 \sqrt{2}$

então, o sistema localizado neste equilibrio é equivalente a:

$$
\left\{\begin{array}{lc}
\dot{x}= & y+\mathcal{O}\left(|(x, y)|^{5}\right) \\
\dot{y}= & d 11 x y-x^{3}-x^{2} y+f x^{3} y+\mathcal{O}\left(|(x, y)|^{5}\right)
\end{array}\right.
$$

onde d11 > 0. Neste caso, dependendo do valor de d11, teremos uma bifurcação do tipo foco nilpotente ou uma do tipo elíptica nilpotente (ver apêndice). 


\subsection{Estudo algébrico das condições de não-degenerescência.}

Para começar este estudo, devemos localizar os pontos onde a bifurcação do tipo BogdanovTakens pode degenerar. Já sabemos que isso ocorre quando uma das seguintes condições ocorrem.

$$
\begin{aligned}
& a 20\left(\alpha_{0}, \beta_{0}, \mu_{0}, \delta_{0}, \lambda_{0}\right)=0 \\
& a 11\left(\alpha_{0}, \beta_{0}, \mu_{0}, \delta_{0}, \lambda_{0}\right)=0
\end{aligned}
$$

Agora, pela primeira seç̧ão, sabemos que para um ponto $\left(x_{0}, y_{0}, \alpha_{0}, \beta_{0}, \mu_{0}, \delta_{0}, \lambda_{0}\right)$ ser candidato a bifurcação Bogdanov-Takens devemos ter: $y_{0}=y_{B T 1}\left(x_{0}, \delta_{0}, \lambda_{0}\right), \alpha_{0}=A\left(x_{0}, y_{B T 1}, \beta \operatorname{tr}, \delta_{0}, \lambda_{0}\right)$, $\mu_{0}=M\left(x_{0}, y_{B T 1}, \beta t r, \delta_{0}, \lambda_{0}\right)$ e $\beta_{0}=\beta_{t r}\left(x_{0}, y_{B T 1}, \delta_{0}, \lambda_{0}\right)$. Assim, podemos fazer essas substituições e as funções $a 20$ e a11 passam a depender apenas de três parâmetros: $x, \delta$ e $\lambda$.

Tomando a mudança de coordenadas $z=\lambda x$, as duas expressões passam a ser lineares em $x$. De fato, temos:

$$
\begin{aligned}
a 11 & =\frac{A_{1}(z, \delta) x+A_{2}(z, \delta)}{x^{2}\left(2 z-1-3 \delta+\sqrt{4 z^{2}-4 z-12 z \delta+1+6 \delta+\delta^{2}}\right)^{3}(z-1)} \\
a 20 & =\delta \frac{B_{1}(z, \delta) x+B_{2}(z, \delta)}{x^{2}\left(2 z-1-3 \delta+\sqrt{4 z^{2}-4 z-12 z \delta+1+6 \delta+\delta^{2}}\right)^{2}(z-1)}
\end{aligned}
$$

Note que o denominador de ambas as expressões são diferentes de zero caso $0<z \leq \frac{1}{2}$. Queremos resolver essas expressões em função de $x$. Para isso, $A_{1}$ e $B_{1}$ não devem se anular:

Lema 7.6.1. Para $\delta>0, A_{1}$ não se anula para $0<z \leq \frac{1}{2}$ e $B_{1}$ não se anula para $0<z<\frac{1}{2}$. Além disso, quando $z=\frac{1}{2}$, a20 não se anula.

Demonstração. Comecemos notando que:

$$
A_{1}=p_{1}(z, \delta)+p_{2}(z, \delta) \sqrt{4 z^{2}-4 z-12 z \delta+1+6 \delta+\delta^{2}}
$$

se encaixa nas condições do lema 7.1.1. Assim, a condição [i] é igual a:

$$
\left(p_{1}\right)^{2}-\left(p_{2}\right)^{2}\left(4 z^{2}-4 z-12 z \delta+1+6 \delta+\delta^{2}\right)=0
$$

o que significa que:

$$
\begin{gathered}
0=8 \delta^{3}(-1+2 z) \\
\left(24 z^{4}-48 z^{3}-12 z^{3} \delta+18 \delta z^{2}+2 z^{2} \delta^{2}+44 z^{2}-20 z-2 z \delta^{2}-14 z \delta+4+\delta^{2}+4 \delta\right)
\end{gathered}
$$


Como $\delta>0$ e $z<1$, temos duas opções: ou $z=\frac{1}{2}$ ou devemos ter $\delta$ igual a:

$$
\begin{aligned}
& d 1=\frac{6 z^{3}-9 z^{2}+7 z-2+\sqrt{-12 z^{6}+36 z^{5}-43 z^{4}+26 z^{3}-7 z^{2}}}{2 z^{2}-2 z+1} \\
& d 2=\frac{6 z^{3}-9 z^{2}+7 z-2-\sqrt{-12 z^{6}+36 z^{5}-43 z^{4}+26 z^{3}-7 z^{2}}}{2 z^{2}-2 z+1}
\end{aligned}
$$

No entanto, como $-12 z^{6}+36 z^{5}-43 z^{4}+26 z^{3}-7 z^{2}<0$ para $0<z \leq \frac{1}{2}$, então $d 1$ e $d 2$ são complexos. Dessa forma, só resta o caso $z=\frac{1}{2}$. Substituindo em $A_{1}$ obtemos $-\delta^{2} \neq 0$. Assim, $A_{1}$ não se anula para $\delta>0$ e $0<z \leq \frac{1}{2}$.

Agora, note que:

$$
B_{1}=q_{1}(z, \delta)+q_{2}(z, \delta) \sqrt{4 z^{2}-4 z-12 z \delta+1+6 \delta+\delta^{2}}
$$

Procedendo da mesma forma, obtemos que a condição [i] equivale a:

$$
\begin{gathered}
0=256 \delta^{4}(-1+2 z)^{2} \\
\left(24 z^{4}-48 z^{3}+12 z^{3} \delta+41 z^{2}-18 \delta z^{2}+z^{2} \delta^{2}-17 z+10 z \delta-z \delta^{2}-2 \delta+3\right)
\end{gathered}
$$

Assim, como $\delta>0$ : ou $z=\frac{1}{2}$ ou $\delta$ é igual a:

$$
\begin{aligned}
& d 1=\frac{-6 z^{3}+9 z^{2}-5 z+1+\sqrt{12 z^{6}-36 z^{5}+52 z^{4}-44 z^{3}+23 z^{2}-7 z+1}}{z(z-1)} \\
& d 2=\frac{-6 z^{3}+9 z^{2}-5 z+1-\sqrt{12 z^{6}-36 z^{5}+52 z^{4}-44 z^{3}+23 z^{2}-7 z+1}}{z(z-1)}
\end{aligned}
$$

Apenas $d 2$ é positivo. Assim, ele inicialmente é um candidato. No entanto, substituindo ele na expressão de $B_{1}$ obtemos uma função que só depende de $z$. Agora a condição [ii] do lema 7.1.1 equivale a:

$$
q_{1}(z, \delta) q_{2}(z, \delta)<0
$$

Substituindo $\delta$ por $d 2$, obtemos que $q_{1}(z, d 1) q_{2}(z, d 2)$ só se anula em $z=\frac{1}{2}$. Avaliando em $0<z<\frac{1}{2}$, obtemos um valor positivo, e como a função é contínua chegamos a conclusão que para $0<z<\frac{1}{2}$ a condição [ii] não é satisfeita.

Assim, resta o caso no qual $z=\frac{1}{2}$. De fato, neste caso $B_{1}=0$. No entanto $a 20\left(x, \frac{1}{2}, \delta\right)=$ $\frac{\delta^{2}}{x^{2}} \neq 0$. Assim, para $z=\frac{1}{2}$, a20 não se anula.

Dessa forma, podemos resolver as equações $a 11=0$ e $a 20=0$ em função de x: 
Definição 7.6.2. Para cada $\delta>0$ e $0<z \leq \frac{1}{2}$ fixados existe um único $x(z, \delta)$ que anula a11 que chamaremos de $x_{B T 3}(z, \delta)$. Para cada $\delta>0$ e $0<z<\frac{1}{2}$ fixados existe um único $x(z, \delta)$ que anula a20 que chamaremos de $x_{N 3}(z, \delta)$.

e temos o seguinte corolário:

Corolário 7.6.3. Para $\delta>0$ :

- $a 20(x, z, \delta)=0$ se, e somente se, $x=x_{N 3}$.

- $a 11(x, z, \delta)=0$ se, e somente se, $x=x_{B T 3}$.

Assim, localizamos os pontos onde podem ocorrer bifurcações mais degeneradas. Agora, temos a intenção de mostrar que tais pontos não podem ser ainda mais degenerados.

Lema 7.6.4. $x_{B T 3}(z, \delta) \neq x_{N 3}(z, \delta)$ qualquer $\delta>0$ e $0<z<\frac{1}{2}$

Demonstração. Tomando o numerador de $x_{N 3}-x_{B T 3}$ obtemos:

$$
x_{N 3}-x_{B T 3}=p(z, \delta)+q(z, \delta) \sqrt{4 z^{2}-4 z-12 z \delta+1+6 \delta+\delta^{2}}
$$

Estamos na condição do lema 7.1.1. Assim, a condição [i] equivale a:

$$
\begin{gathered}
16 \delta^{6}(z-1)^{4} \\
\left(24 z^{3} \delta-6 z^{2}-2 z^{2} \delta^{2}-36 \delta z^{2}+6 z+2 z \delta^{2}+24 z \delta-1-6 \delta-\delta^{2}\right)=0
\end{gathered}
$$

Como $z<\frac{1}{2}$ e $\delta>0$, devemos ter:

$$
\left(24 z^{3} \delta-6 z^{2}-2 z^{2} \delta^{2}-36 \delta z^{2}+6 z+2 z \delta^{2}+24 z \delta-1-6 \delta-\delta^{2}\right)=0
$$

Resolvendo em função de $\delta$ obtemos:

$$
\begin{aligned}
& d 1=\frac{12 z^{3}-18 z^{2}+12 z-3+2 \sqrt{36 z^{6}-108 z^{5}+150 z^{4}-120 z^{3}+58 z^{2}-16 z+2}}{1-2 z+2 z^{2}} \\
& d 2=\frac{12 z^{3}-18 z^{2}+12 z-3-2 \sqrt{36 z^{6}-108 z^{5}+150 z^{4}-120 z^{3}+58 z^{2}-16 z+2}}{1-2 z+2 z^{2}}
\end{aligned}
$$

sendo que $d 2<0$. Assim, apenas $d 1$ é um candidato válido. No entanto, $d 1$ só é válido para $z>1 / 2-1 / 6 \sqrt{3}$. Tomando a condição [ii], não é difícil mostrar que $p(z, d 1) q(z, d 1)$ não se anula para $z \in] 1 / 2-1 / 6 \sqrt{3}, 1 / 2[\mathrm{e}$, calculando em qualquer ponto, temos que essa expressão é positiva e portanto a condição [ii] não é válida.

Dessa forma, $x_{B T 3} \neq x_{N 3}$. 
Já sabemos que a20 e a11 não podem se anular simultaneamente. Assim, devemos nos preocupar com as degenerescências quando cada um desses coeficientes se anula. Vamos começar com o caso $a 11=0$. Na secção 3, concluímos que a condição de não-degenerescência para esse caso é igual a função $E$ ser diferente de zero. Lembremos que:

$$
E=\text { a21 a02 a20 - a30 a21 - } 3 \text { a03 a20 } 2
$$

Tomando: $y_{0}=y_{B T 1}\left(x_{0}, \delta_{0}, \lambda_{0}\right), \alpha_{0}=A\left(x_{0}, y_{B T 1}, \beta t r, \delta_{0}, \lambda_{0}\right), \mu_{0}=M\left(x_{0}, y_{B T 1}, \beta t r, \delta_{0}, \lambda_{0}\right)$ e $\beta_{0}=\beta_{t r}\left(x_{0}, y_{B T 1}, \delta_{0}, \lambda_{0}\right)$, obtemos uma expressão que depende apenas de três variáveis para $E$. Fazendo a mudança de coordenadas $z=x \lambda$, podemos substituir $x$ por $x_{B T 3}$, já que apenas neste caso $E$ nós interessa. O denominador de $E$ é sempre não nulo para $\delta>0$ e $0<z \leq \frac{1}{2}$. Assim, resta estudar quando que o numerador se anula. Conjecturamos que ele nunca se anula. Comecemos restringindo as possibilidades:

Lema 7.6.5. Tome $(x, y, \alpha, \beta, \mu, \delta, \lambda)$ um candidato a bifurcação de Bogdanov-Takens tal que, $a 11=0$. Neste caso, se:

$$
\begin{gathered}
p o l=\left(4 z^{2}-4 z+2\right) \delta^{4}+\left(-66 z^{3}+102 z^{2}-65 z+16\right) \delta^{3}+ \\
\left(78 z^{4}-156 z^{3}+125 z^{2}-56 z+11\right) \delta^{2}+\left(-24 z^{5}+114 z^{4}+2-108 z^{3}+48 z^{2}-13 z\right) \delta \\
-36 z^{5}+12 z^{4}-6 z^{3} \neq 0
\end{gathered}
$$

onde $z=x \lambda$, então $E \neq 0$ e as condições de não-degenerescência são satisfeitas.

Demonstração. Comecemos tomando o numerador de $E$ :

$$
p(z, \delta)+q(z, \delta) \sqrt{4 z^{2}-12 z \delta-4 z+\delta^{2}+6 \delta+1}
$$

Que claramente respeita as condições do lema 7.1.1. Assim, para a condição [i] ser satisfeita, devemos ter:

$$
p^{2}-q^{2}\left(4 z^{2}-12 z \delta-4 z+\delta^{2}+6 \delta+1\right)=0
$$

Tal condição implica que:

$$
\begin{aligned}
& \text { - } \delta=\frac{6 z^{3}-9 z^{2}+7 z-2+\sqrt{-12 z^{6}+36 z^{5}-43 z^{4}+26 z^{3}-7 z^{2}}}{2 z^{2}-2 z+1} \\
& \text { - } \delta=\frac{6 z^{3}-9 z^{2}+7 z-2-\sqrt{-12 z^{6}+36 z^{5}-43 z^{4}+26 z^{3}-7 z^{2}}}{2 z^{2}-2 z+1}
\end{aligned}
$$




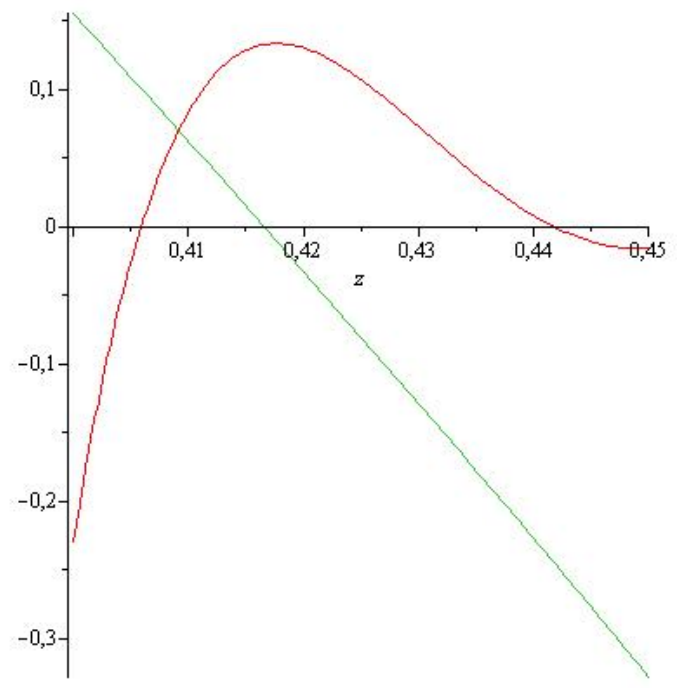

Figura 7.7: Em verde a função pol e em vermelho $100 p(z, \delta) q(z, \delta)$. Note que $z$ está restrito apenas a $0.4<z<0.45$. Este gráfico foi feito $\operatorname{com} \delta=\frac{1}{2}$.

- $\left(2 z^{3}-4 z^{2}+3 z-1\right) \delta^{4}+\left(8 z^{4}-8 z^{3}+5 z-2\right) \delta^{3}+\left(8 z^{5}-32 z^{4}+24 z^{3}-4 z^{2}+z-1\right) \delta^{2}+$ $\left(-48 z^{5}+64 z^{4}-32 z^{3}+8 z^{2}-z\right) \delta+32 z^{6}-24 z^{5}+8 z^{4}-2 z^{3}=0$

- $\left(4 z^{2}-4 z+2\right) \delta^{4}+\left(-66 z^{3}+102 z^{2}-65 z+16\right) \delta^{3}+\left(78 z^{4}-156 z^{3}+125 z^{2}-56 z+11\right) \delta^{2}+$ $\left(-24 z^{5}+114 z^{4}+2-108 z^{3}+48 z^{2}-13 z\right) \delta-36 z^{5}+12 z^{4}-6 z^{3}=0$

os dois primeiros são facilmente descartados uma vez que $\delta$ é complexo para $0<z \leq \frac{1}{2}$. O terceiro também pode ser descartado pois todos os coeficientes são negativos para $0<z \leq \frac{1}{2}$ e portanto não existe raiz com $\delta$ positivo. Assim, resta apenas a última igualdade:

$$
\begin{gathered}
p o l=\left(4 z^{2}-4 z+2\right) \delta^{4}+\left(-66 z^{3}+102 z^{2}-65 z+16\right) \delta^{3}+ \\
\left(78 z^{4}-156 z^{3}+125 z^{2}-56 z+11\right) \delta^{2}+\left(-24 z^{5}+114 z^{4}+2-108 z^{3}+48 z^{2}-13 z\right) \delta \\
-36 z^{5}+12 z^{4}-6 z^{3}=0
\end{gathered}
$$

Tal polinômio pode se anular em parâmetros válidos.

O lema anterior diminui o conjunto onde $E(z, \delta)$ pode se anular. Conjecturamos que $E(z, \delta)$ nunca se anula para parâmetros válidos. Isso se baseia em um estudo numérico. A dificuldade de uma demonstração algébrica é que não conseguimos encontrar explicitamente o ponto onde $p o l=0$ e o intervalo onde $p(z, \delta) q(z, \delta)>0$ normalmente é muito pequeno, como 
vemos na figura 7.7. Se aceitarmos a conjectura, concluímos que a maior degenerescência é de codimensão 3 .

Agora, vamos estudar as condições de não-degenerescência relativas à bifurcação que ocorre quando $a 20=0$. Já sabemos que $a 11 \neq 0$ e portanto estamos nas condições da quarta secção. A primeira condição é $F 1 \neq 0$. Assim, como no caso anterior, começamos substituindo: $y_{0}=y_{B T 1}\left(x_{0}, \delta_{0}, \lambda_{0}\right), \alpha_{0}=A\left(x_{0}, y_{B T 1}, \beta t r, \delta_{0}, \lambda_{0}\right), \mu_{0}=M\left(x_{0}, y_{B T 1}, \beta t r, \delta_{0}, \lambda_{0}\right)$ e $\beta_{0}=\beta_{t r}\left(x_{0}, y_{B T 1}, \delta_{0}, \lambda_{0}\right)$. Obtemos uma expressão para $F 1$ que só depende de $x, \delta$ e $\lambda$. Tomando $z=x \lambda$ e tomando $x=x_{N 3}$ obtemos uma expressão para $F 1$ que só depende de $z$ e $\delta$. Nossa intenção é mostrar que $F 1(z, \delta) \neq 0$ para $\delta>0$ e $0<z<\frac{1}{2}$.

Lema 7.6.6. Sobre um ponto que é candidato a bifurcação de Bogdanov-Takens e que a20 = 0 , temos que $F 1 \neq 0$. Mais especificamente, $F 1<0$.

Demonstração. Para começar note que o denominador de $F 1$ é sempre não nulo para $\delta>0$ e $0<z<\frac{1}{2}$. Assim, devemos estudar quando o numerador se anula. O numerador vale:

$$
\begin{gathered}
16\left(p_{1}\left(z, \delta+q_{2}(z, \delta) \sqrt{4 z^{2}-4 z-12 z \delta+1+6 \delta+\delta^{2}}\right)^{2}\right. \\
\left(p_{2}(z, \delta)+q_{2}(z, \delta) \sqrt{4 z^{2}-4 z-12 z \delta+1+6 \delta+\delta^{2}}\right)
\end{gathered}
$$

Como $\delta>0, z<\frac{1}{2}$ e estamos nas condições do lema 7.1.1, a condição [i] equivale a:

$$
p_{i}(z, \delta)^{2}-q_{i}(z, \delta)^{2}\left(4 z^{2}-4 z-12 z \delta+1+6 \delta+\delta^{2}\right)=0
$$

para $i=1$ ou 2 .

Resolvendo $p_{1}(z, \delta)^{2}-q_{1}(z, \delta)^{2}\left(4 z^{2}-4 z-12 z \delta+1+6 \delta+\delta^{2}\right)=0$ em função de $\delta$, obtemos que $\delta$ tem duas soluções, sendo uma delas negativa. A solução positiva vale:

$$
d 1=-\frac{6 z^{3}-9 z^{2}+5 z-1+\sqrt{12 z^{6}-36 z^{5}+52 z^{4}-44 z^{3}+23 z^{2}-7 z+1}}{z(-1+z)}
$$

No entanto não é difícil mostrar que $p_{1}(z, d 1) q_{1}(z, d 1)>0$ para $\left.z \in\right] 0, \frac{1}{2}[$. Logo esta parte da expressão de $F 1$ não se anula.

Já quando resolvemos $p_{2}(z, \delta)^{2}-q_{2}(z, \delta)^{2}\left(4 z^{2}-4 z-12 z \delta+1+6 \delta+\delta^{2}\right)=0$ obtemos que: 
- $\delta=0$

- $\delta=\frac{z}{1-z}$

- $\left(z^{2}-z\right) \delta^{3}+\left(20 z^{3}-23 z^{2}+10 z-2\right) \delta^{2}+\left(-14 z^{3}+5 z^{2}+16 z^{4}-z\right) \delta+16 z^{5}+6 z^{3}-$ $16 z^{4}-z^{2}=0$

O primeiro caso pode ser descartado. Note que, no terceiro caso, todos os coeficientes do polinômio em $\delta$ são negativos para $0<z<\frac{1}{2}$. Dessa forma, fixando $z$, ele não possui raízes positivas e pode ser descartado. Assim, resta apenas o caso em que $\delta=\frac{z}{1-z}$. No entanto, considerando a condição [ii] temos:

$$
16 \frac{z^{20}(2 z-1)^{2}\left(2 z^{2}-1\right)\left(4 z^{5}-16 z^{4}+26 z^{3}-18 z^{2}+6 z-1\right)^{2}}{(-1+z)^{17}}>0
$$

Assim sendo, $F 1$ não se anula.

Como $F 1(z, \delta)$ nunca se anula e é uma função contínua e bem definida em um conjunto conexo, ela deve ter sempre o mesmo sinal. Calculando ele em qualquer ponto, obtemos que ele é negativo.

Agora, vamos tratar da condição $F 2 \neq 0$. Lembremos que $F 2$ vale:

$$
\begin{gathered}
F 2=b 21\|b 30\|-\frac{3}{5} b 40 b 11= \\
=(5 / 2|a 30| a 11 \text { a02 }+5 \mid \text { a30|a21 }-3 / 2 \text { a11 a02 a30 })\left(\alpha_{0}, \beta_{0}, \mu_{0}, \delta_{0}, \lambda_{0}\right)
\end{gathered}
$$

Pelo lema anterior $a 30<0$ e, portanto, temos que $F 2 \neq 0$ se, e somente se, (4a11a02$5 a 21)\left(\alpha_{0}, \beta_{0}, \mu_{0}, \delta_{0}, \lambda_{0}\right) \neq 0$. Assim, chegamos no seguinte lema:

Lema 7.6.7. Seja $\left(x, y, \alpha_{0}, \beta_{0}, \mu_{0}, \delta_{0}, \lambda_{0}\right)$ um ponto candidato a bifurcação de BogdanovTakens no qual a20 $\left(\alpha_{0}, \beta_{0}, \mu_{0}, \delta_{0}, \lambda_{0}\right)=0$. Caso:

$$
\begin{gathered}
\left(40+130 z^{2}-130 z\right) \delta^{4}+\left(2442 z^{2}-1243 z+242-1743 z^{3}\right) \delta^{3}+ \\
\left(-11577 z^{3}-3022 z+427+8563 z^{2}+5901 z^{4}\right) \delta^{2}+ \\
\left(2346 z^{2}+1860 z^{5}-671 z+930 z^{4}-3459 z^{3}+52\right) \delta+195 z^{3}-615 z^{4}+900 z^{5} \neq 0
\end{gathered}
$$

onde $z=x \lambda$. Então, F2 é não nulo. 
Demonstração. Já sabemos que para $F 2$ se anular, devemos ter

$$
(4 a 11 a 02-5 a 21)\left(x, y, \alpha_{0}, \beta_{0}, \mu_{0}, \delta_{0}, \lambda_{0}\right)=0
$$

Tomando essa expressão e substituindo $y_{0}$ por $y_{B T 1}, \beta_{0}$ por $\beta_{t r}, \alpha_{0}$ por $A$ e $\mu_{0}$ por $M$ obtemos uma expressão que depende apenas de $x, \delta$ e $\lambda$. Tomando $z=x \lambda$ e substituindo $x$ por $x_{N 3}$ obtemos uma expressão que só depende de $z$ e $\delta$. Assim, o numerador de F2 vale:

$$
\begin{gathered}
a 30\left(-8\left(p_{1}(z, \delta)+q_{1}(z, \delta) \sqrt{4 z^{2}-4 z-12 z \delta+1+6 \delta+\delta^{2}}\right)\right. \\
\left.\left(p_{2}(z, \delta)+q_{2}(z, \delta) \sqrt{4 z^{2}-4 z-12 z \delta+1+6 \delta+\delta^{2}}\right)^{2}\right)
\end{gathered}
$$

o denominador de F2 é não nulo e portanto não altera a análise. Basta verificar quando o numerador se anula. Assim, como para existir $x_{N 3}$ devemos ter $0<z<\frac{1}{2}$ e estamos nas condições do lema 7.1.1, uma das duas condições deve ocorrer:

$$
p_{i}(z, \delta)^{2}-q_{i}(z, \delta)^{2}\left(4 z^{2}-4 z-12 z \delta+1+6 \delta+\delta^{2}\right)=0
$$

para $i$ igual a 1 ou 2. No caso em que consideramos $i=2$, essa equação nos gera:

$$
24 z^{4}+(12 \delta-48) z^{3}+\left(-18 \delta+41+\delta^{2}\right) z^{2}+\left(-\delta^{2}-17+10 \delta\right) z-2 \delta+3=0
$$

resolvendo em função de $\delta$, obtemos duas soluções, sendo que uma delas é sempre negativa. Assim, a única possibilidade de solução é:

$$
\delta=d 1=-\frac{6 z^{3}-9 z^{2}+5 z-1+\sqrt{12 z^{6}-36 z^{5}+52 z^{4}-44 z^{3}+23 z^{2}-7 z+1}}{z(-1+z)}
$$

No entanto, ao considerarmos a condição [ii], podemos mostrar que $p_{2}(z, d 1) q_{2}(z, d 1)$ não possui soluções para $z \in] 0, \frac{1}{2}$ [ e é sempre positiva neste intervalo. Logo não temos soluções provenientes desta parte da expressão de $F 2$.

Já quando trabalhamos com a condição $i=1$, obtemos:

$$
16 \delta^{8} \operatorname{pol}(z, \delta)
$$

onde:

$$
\begin{gathered}
\operatorname{pol}(z, \delta)=\left(40+130 z^{2}-130 z\right) \delta^{4}+\left(2442 z^{2}-1243 z+242-1743 z^{3}\right) \delta^{3}+ \\
\left(-11577 z^{3}-3022 z+427+8563 z^{2}+5901 z^{4}\right) \delta^{2}+ \\
+\left(2346 z^{2}+1860 z^{5}-671 z+930 z^{4}-3459 z^{3}+52\right) \delta+195 z^{3}-615 z^{4}+900 z^{5} .
\end{gathered}
$$


Assim, apenas se $\operatorname{pol}(z, \delta)=0$ a condição [i] do lema 7.1.1 é satisfeita e tem-se chance do termo $F 2$ ser igual a zero.

Nesta última demonstração restringimos o conjunto de equilíbrios e parâmetros onde podemos ter uma bifurcação nilpotente com $a 20=0$ e $F 2=0$. No entanto, conjecturamos que este conjunto é vazio. De fato, a condição [ii] do lema 7.1.1 precisa ser verificada. Já tivemos um problema parecido para delimitarmos quando $E$ era igual a zero. Lá conjecturamos baseados em indícios numéricos que $E$ era sempre diferente de zero. Neste caso, novos indícios numéricos nos levam a conjecturar que $F 2$ é sempre diferente de zero. Acreditamos que se acharmos um método para provar uma das duas conjecturas, o mesmo método provará a outra.

Antes de continuarmos com a próxima condição de não-degenerescência é importante notar que: como a11 é uma função contínua não nula e bem definida em um conjunto conexo, ela permanece com o mesmo sinal. Substituindo em qualquer ponto, obtemos que este sinal é negativo.

Resta agora estudar a condição $F 3$. Lembremos que deveríamos ter:

$$
F 3=\left\|\frac{b 11}{\sqrt{\|b 30\|}}\right\|=\left\|\frac{a 11}{\sqrt{\|a 30\|}}\right\| \neq 2 \sqrt{2}
$$

Já sabemos que a30 é sempre negativo sobre o conjunto que agora nos interessa. Assim sendo, devemos ter:

$$
-\frac{a 11}{\sqrt{-a 30}} \neq 2 \sqrt{2}
$$

Substituindo $y_{0}$ por $y_{B T 1}, \beta_{0}$ por $\beta_{t r}, \alpha_{0}$ por $A$ e $\mu_{0}$ por $M$ obtemos uma expressão que depende apenas de $x, \delta$ e $\lambda$. Tomando $z=x \lambda$ e substituindo $x$ por $x_{N 3}$ obtemos uma expressão que só depende de $z$ e $\delta$. Assim, devemos ter:

$$
-\frac{a 11}{\sqrt{-a 30}}\left(z, \delta_{0}\right) \neq 2 \sqrt{2}
$$

Esta não é um condição verdadeira para todo $\delta>0$ e $0<z<\frac{1}{2}$. Isso pode ser verificado graficamente através da figura 7.8. Neste trabalho não temos a intenção de estudar o que 


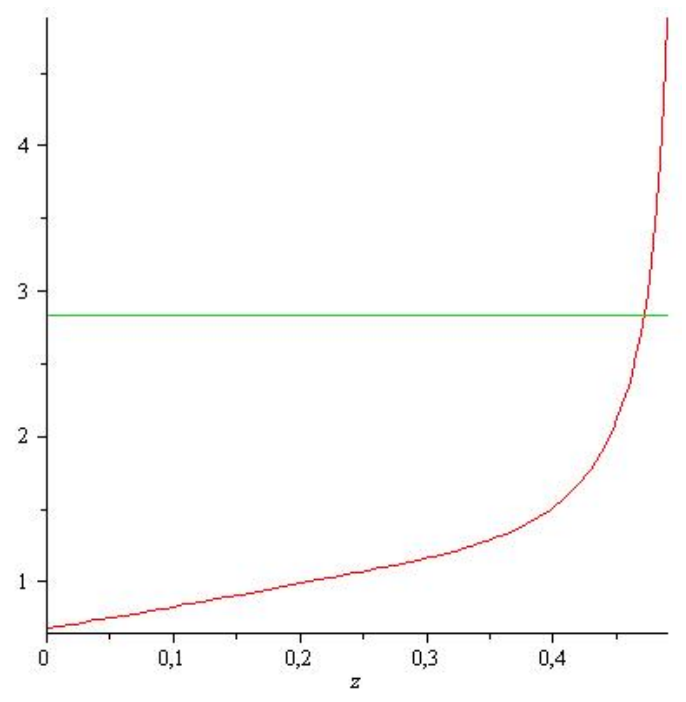

Figura 7.8: Fixamos $\delta=\frac{1}{2}$. Em vermelho temos o gráfico de $F 3$ e em verde a gráfico constante $2 \sqrt{2}$. Note que o cruzamento se dá para $z$ bem próximo de $\frac{1}{2}$. Isso leva em uma bifurcação de codimensão quatro de transição entre um foco nilpotente e um elíptico nilpotente, ainda não estudada.

ocorre quando a condição F3 falha. Através de um cálculo numérico, notamos que para cada $\delta$ fixado, temos duas situações possíveis: $F 3-2 \sqrt{2}$ se anula uma ou duas vezes. Isso implica que para cada $\delta$ existe pelo menos um $\lambda$ e no máximo dois $\lambda$ 's onde ocorre uma transição entre um foco nilpotente e um elíptico nilpotente. Mostraremos em uma análise subsequente que tal bifurcação ocorre para parâmetros válidos e, portanto, deve ser considerada no diagrama de bifurcação. Até o presente momento, não é de nosso conhecimento nenhum estudo acerca desta bifurcação.

Assim, podemos reunir os principais resultados da secção nas seguintes proposições (usando o lema 7.1.2 para enuncia-los):

Proposição 7.6.8. Existe um conjunto aberto e denso contido em $\Delta$, que chamaremos de $A$, tal que para todo $\left(\delta_{0}, \lambda_{0}\right) \in A$, caso $\left(x_{0}, y_{0}\right)$ com parâmetros $\left(\alpha_{0}, \beta_{0}, \mu_{0}, \delta_{0}, \lambda_{0}\right)$ seja um equilíbrio com jacobiano nilpotente e a11( $\left.x_{0}, y_{0}, \alpha_{0}, \beta_{0}, \mu_{0}, \delta_{0}, \lambda_{0}\right)=0$, então o sistema 
localizado no equilíbrio é equivalente a:

$$
\left\{\begin{array}{c}
\dot{x}=y+\mathcal{O}\left(\|(x, y)\|^{5}\right) \\
\dot{y}=x^{2}+E x^{3} y+\mathcal{O}\left(\|(x, y)\|^{5}\right)
\end{array}\right.
$$

com $E \neq 0$.

Proposição 7.6.9. Existe um conjunto aberto e denso contido em $\Delta$, que chamaremos de $B$, tal que para todo $\left(\delta_{0}, \lambda_{0}\right) \in B$, caso $\left(x_{0}, y_{0}\right)$ com parâmetros $\left(\alpha_{0}, \beta_{0}, \mu_{0}, \delta_{0}, \lambda_{0}\right)$ seja um equilíbrio com jacobiano nilpotente e a $20\left(x_{0}, y_{0}, \alpha_{0}, \beta_{0}, \mu_{0}, \delta_{0}, \lambda_{0}\right)=0$, então o sistema localizado no equilíbrio é equivalente a:

$$
\left\{\begin{array}{c}
\dot{x}=y+\mathcal{O}\left(\|(x, y)\|^{5}\right) \\
\dot{y}=-x^{3}+d 11 x y+d 21 x^{2} y+d 31 x^{3} y+\mathcal{O}\left(\|(x, y)\|^{5}\right)
\end{array}\right.
$$

com $d 11<0$ e $d 21 \neq 0$.

Além disso, através de indícios numéricos, chegamos às seguintes conjecturas:

- $A=\Delta$

- $B=\Delta$

- Podemos melhorar a segunda proposição de forma que o sistema seja equivalente a:

$$
\left\{\begin{array}{c}
\dot{x}=y+\mathcal{O}\left(\|(x, y)\|^{5}\right) \\
\dot{y}=-x^{3}+d 11 x y-x^{2} y+d 31 x^{3} y+\mathcal{O}\left(\|(x, y)\|^{5}\right)
\end{array}\right.
$$

$\operatorname{com} d 11>0$

Por fim, também concluímos que existe $\delta>0$ e $0<z<\frac{1}{2}$ tal que $F 3=2 \sqrt{2}$ e, portanto, temos uma bifurcação mais degenerada.

\subsection{Condições de transversalidade}

Nesta secção, vamos tratar das condições de transversalidade dos candidatos a bifurcações do tipo Bogdanov-Takens e nilpotentes mais degenerados. Começamos este estudo pelos 
candidatos a bifurcação Bogdanov-Takens que não sejam mais degenerados. Pela primeira secção, sabemos que os pontos $(x, y, \alpha, \beta, \mu, \delta, \lambda)$ que são candidatos estão localizados em uma curva: $\left(x, y_{B T 1}, A, \beta_{t r}, M, \delta, \lambda\right)$.

Lema 7.7.1. Para os pontos $(x, y, \alpha, \beta, \mu, \delta, \lambda)$ candidatos a bifurcação de Bogdanov-Takens tais que $a 11 \neq 0 \neq a 20$, ocorre uma bifurcação do tipo Bogdanov-Takens.

Demonstração. A condição de transversalidade relativa a este tipo de bifurcação é:

$$
\operatorname{Det}\left(\mathcal{J}_{x, y, \gamma}\left(f_{1}, f_{2}, \operatorname{Det}\left(\mathcal{J}_{x, y}\left(f_{1}, f_{2}\right)\right), \operatorname{Tr}\left(\mathcal{J}_{x, y}\left(f_{1}, f_{2}\right)\right)\right)(x, y, \alpha, \beta, \mu, \delta, \lambda) \neq 0\right.
$$

onde $\gamma \in \mathbb{R}^{2}$ é o parâmetro. Para o cálculo das condições de transversalidade, vamos escolher $\gamma=(\alpha, \beta)$. Assim:

$$
C 1=\operatorname{Det}\left(\mathcal{J}_{x, y, \alpha, \beta}\left(f_{1}, f_{2}, \operatorname{Det}\left(\mathcal{J}_{x, y}\left(f_{1}, f_{2}\right)\right), \operatorname{Tr}\left(\mathcal{J}_{x, y}\left(f_{1}, f_{2}\right)\right)\right)(x, y, \alpha, \beta, \mu, \delta, \lambda)\right.
$$

quando calculamos sobre a curva $\left(x, y_{B T 1}, A, \beta_{t r}, M, \delta, \lambda\right)$ e fazemos a mudança de coordenadas $z=x \lambda$ obtemos:

$$
\frac{16 x \delta^{7}(z-1)\left(p(z, \delta)+q(z, \delta) \sqrt{4 z^{2}-12 z \delta-4 z+\delta^{2}+6 \delta+1}\right)}{\left(2 z-3 \delta-1+\sqrt{4 z^{2}-12 z \delta-4 z+\delta^{2}+6 \delta+1}\right)^{7}}
$$

onde $\delta>0$ e $0<z \leq \frac{1}{2}$. Dessa forma a única forma dessa expressão se anular é as condições [i] e [ii] do lema 7.1.1 serem satisfeitas. Comecemos pela condição [i]:

$$
p(z, \delta)^{2}-q(z, \delta)^{2}\left(4 z^{2}-12 z \delta-4 z+\delta^{2}+6 \delta+1\right)=0
$$

no entanto essa expressão vale:

$$
32 \delta^{7}(-1+2 z)\left(24 z^{3} \delta-2 z^{2} \delta^{2}-6 z^{2}-36 \delta z^{2}+6 z+2 z \delta^{2}+24 z \delta-1-6 \delta-\delta^{2}\right)
$$

Igualando a zero e resolvendo obtemos:

$$
\begin{aligned}
& \text { - } z=\frac{1}{2} \\
& \text { - } \delta=d 1=\frac{12 z^{3}+12 z-18 z^{2}-3+2 \sqrt{36 z^{6}+150 z^{4}-108 z^{5}-120 z^{3}+58 z^{2}-16 z+2}}{2 z^{2}-2 z+1}
\end{aligned}
$$


- $\delta=d 2=\frac{12 z^{3}+12 z-18 z^{2}-3-2 \sqrt{36 z^{6}+150 z^{4}-108 z^{5}-120 z^{3}+58 z^{2}-16 z+2}}{2 z^{2}-2 z+1}$

Como $d 2<0$, podemos descartar esta opção. Agora, tomando $z=\frac{1}{2}$ e substituindo em $C 1$ obtemos:

$$
1 / 4 x \delta^{4} \neq 0
$$

assim, podemos descartar esta opção também. Por fim, devemos considerar a opção [ii]. É fácil ver que $d 1>0$ apenas se $z \in] 1 / 2-1 / 6 \sqrt{3}, \frac{1}{2}[$. Também é fácil ver que $p(z, d 1) q(z, d 1) \neq$ 0 com $z \in] 1 / 2-1 / 6 \sqrt{3}, \frac{1}{2}[$. Avaliando em um ponto, chegamos a conclusão que está expressão é sempre positiva e, portanto, $d 1$ pode ser descartado. Assim sendo, a condição de transversalidade é respeitada e temos uma bifurcação do tipo Bogdanov-Takens.

Agora, vamos tratar do caso do candidato a bifurcação Bogdanov-Takens de codimensão 3.

Lema 7.7.2. Para os pontos $(x, y, \alpha, \beta, \mu, \delta, \lambda)$ candidatos a bifurcação de Bogdanov-Takens, com $a 11=0, E \neq 0$ e

- $(288+384 \delta) z^{7}+(-1080-2280 \delta) z^{6}+\left(5040 \delta+1584+288 \delta^{2}\right) z^{5}+$

$$
\begin{aligned}
& \left(-1308-5214 \delta+2 \delta^{4}-1050 \delta^{2}-18 \delta^{3}\right) z^{4}+\left(3140 \delta+1080 \delta^{2}+696-4 \delta^{4}+120 \delta^{3}\right) z^{3}+ \\
& \left(-1206 \delta-240-611 \delta^{2}-96 \delta^{3}-3 \delta^{4}\right) z^{2}+\left(2 \delta^{4}+206 \delta^{2}+276 \delta+48+44 \delta^{3}\right) z-4- \\
& \delta^{4}-29 \delta^{2}-28 \delta-10 \delta^{3} \neq 0,
\end{aligned}
$$

onde $z=x \lambda$, ocorre uma bifurcação do tipo Bogdanov-Takens de codimensão 3.

Demonstração. A condição de transversalidade relativa a este tipo de bifurcação é:

$$
\operatorname{Det}\left(\mathcal{J}_{x, y, \gamma}\left(f_{1}, f_{2}, \operatorname{Det}\left(\mathcal{J}_{x, y}\left(f_{1}, f_{2}\right)\right), \operatorname{Tr}\left(\mathcal{J}_{x, y}\left(f_{1}, f_{2}\right)\right), a 11\right)(x, y, \alpha, \beta, \mu, \delta, \lambda) \neq 0\right.
$$

onde $\gamma \in \mathbb{R}^{3}$ é o parâmetro. Para o cálculo das condições de transversalidade, vamos escolher $\gamma=(\alpha, \beta, \mu)$. Assim:

$$
C 2=\operatorname{Det}\left(\mathcal{J}_{x, y, \alpha, \beta, \mu}\left(f_{1}, f_{2}, \operatorname{Det}\left(\mathcal{J}_{x, y}\left(f_{1}, f_{2}\right)\right), \operatorname{Tr}\left(\mathcal{J}_{x, y}\left(f_{1}, f_{2}\right)\right), a 11\right)(x, y, \alpha, \beta, \mu, \delta, \lambda)\right.
$$

quando calculamos sobre a curva $\left(x, y_{B T 1}, A, \beta_{t r}, M, \delta, \lambda\right)$, fazemos a mudança de coordenadas $z=x \lambda \mathrm{e}$, por fim, tomamos $x=x_{B T 3}$, obtemos:

$$
C 2=\frac{-64 \delta^{6}(z-1)^{3}\left(p(z, \delta)+q(z, \delta) \sqrt{4 z^{2}-12 z \delta-4 z+\delta^{2}+6 \delta+1}\right)}{\operatorname{denom}(C 2)}
$$


O denominador de C2 não se anula para $\delta>0$ e $0<z \leq \frac{1}{2}$. O numerador se encaixa nas condições do lema 7.1.1. Analisando a condição [i] obtemos:

- $z=\frac{1}{2}$

- $(288+384 \delta) z^{7}+(-1080-2280 \delta) z^{6}+\left(5040 \delta+1584+288 \delta^{2}\right) z^{5}+$ $\left(-1308-5214 \delta+2 \delta^{4}-1050 \delta^{2}-18 \delta^{3}\right) z^{4}+\left(3140 \delta+1080 \delta^{2}+696-4 \delta^{4}+120 \delta^{3}\right) z^{3}+$ $\left(-1206 \delta-240-611 \delta^{2}-96 \delta^{3}-3 \delta^{4}\right) z^{2}+\left(2 \delta^{4}+206 \delta^{2}+276 \delta+48+44 \delta^{3}\right) z-4-$ $\delta^{4}-29 \delta^{2}-28 \delta-10 \delta^{3}=0$

Calculando na primeira opção temos:

$$
C 2\left(\frac{1}{2}, \delta\right)=1 / 8 \delta^{3}(1+3 \delta) \neq 0
$$

Assim, resta apenas a segunda opção.

Tratando deste caso numericamente, temos fortes indícios de que, na verdade, $C 2$ nunca se anule, e, portanto, o resultado é válido para os pontos nos quais o jacobiano é nilpotente e $a 11=0$.

Resta tratar da condição de transversalidade relativa a bifurcação nilpotente mais degenerada. Neste caso, vamos tratar apenas das condições de transversalidade relativas aos focos nilpotentes e aos elípticos nilpotentes, não tratando da bifurcação mais degenerada que encontramos.

Assim, começamos com o seguinte lema:

Lema 7.7.3. Os pontos $(x, y, \alpha, \beta, \mu, \delta, \lambda)$ candidatos a bifurcação de Bogdanov-Takens, com $a 20=0$, cujas condições de degenerescência são satisfeitas e tais que:

$$
\begin{gathered}
(1152+1920 \delta) z^{9}+\left(576 \delta^{2}-5664-11808 \delta\right) z^{8}+\left(11712+288 \delta^{3}-2880 \delta^{2}+30432 \delta\right) z^{7} \\
+\left(-42528 \delta+16 \delta^{4}-13788-936 \delta^{3}+6724 \delta^{2}\right) z^{6}+\left(36276 \delta-8508 \delta^{2}+10380+1148 \delta^{3}-48 \delta^{4}\right) z^{5} \\
+\left(6302 \delta^{2}-20040 \delta-680 \delta^{3}-5245+43 \delta^{4}\right) z^{4}+\left(180 \delta^{3}-2852 \delta^{2}+7312 \delta-6 \delta^{4}+1790\right) z^{3} \\
+\left(-398+791 \delta^{2}+2 \delta^{3}-7 \delta^{4}-1728 \delta\right) z^{2}+\left(-12 \delta^{3}-126 \delta^{2}+2 \delta^{4}+244 \delta+52\right) z \\
+2 \delta^{3}-3+9 \delta^{2}-16 \delta \neq 0,
\end{gathered}
$$

onde $z=x \lambda$, são um foco nilpotente ou um elíptico nilpotente. 
Demonstração. A condição de transversalidade relativa a este tipo de bifurcação é:

$$
\operatorname{Det}\left(\mathcal{J}_{x, y, \gamma}\left(f_{1}, f_{2}, \operatorname{Det}\left(\mathcal{J}_{x, y}\left(f_{1}, f_{2}\right)\right), \operatorname{Tr}\left(\mathcal{J}_{x, y}\left(f_{1}, f_{2}\right)\right), a 20\right)(x, y, \alpha, \beta, \mu, \delta, \lambda) \neq 0\right.
$$

onde $\gamma \in \mathbb{R}^{3}$ é o parâmetro. Para o cálculo das condições de transversalidade, vamos escolher $\gamma=(\alpha, \beta, \mu)$. Assim:

$$
C 3=\operatorname{Det}\left(\mathcal{J}_{x, y, \alpha, \beta, \mu}\left(f_{1}, f_{2}, \operatorname{Det}\left(\mathcal{J}_{x, y}\left(f_{1}, f_{2}\right)\right), \operatorname{Tr}\left(\mathcal{J}_{x, y}\left(f_{1}, f_{2}\right)\right), a 20\right)(x, y, \alpha, \beta, \mu, \delta, \lambda)\right.
$$

quando calculamos sobre a curva $\left(x, y_{B T 1}, A, \beta_{t r}, M, \delta, \lambda\right)$, fazemos a mudança de coordenadas $z=x \lambda$ e, por fim, tomamos $x=x_{N 3}$, obtemos:

$$
C 3=\frac{64 \delta^{7}(z-1)^{3}\left(p(z, \delta)+q(z, \delta) \sqrt{4 z^{2}-4 z-12 z \delta+1+6 \delta+\delta^{2}}\right)}{\operatorname{denom}(C 3)}
$$

Assim, para que $C 3 \neq 0$ uma condição suficiente é [i] do lema 7.1.1 não ser satisfeita:

$$
p(z, \delta)^{2}-q(z, \delta)^{2}\left(4 z^{2}-4 z-12 z \delta+1+6 \delta+\delta^{2}\right) \neq 0
$$

O que nos fornece a seguinte condição:

$$
32 \delta^{9}(-1+2 z) \operatorname{pol}(z, \delta) \neq 0
$$

Como $\delta>0$ e $0<z<\frac{1}{2}$. Dessa forma, basta que $\operatorname{pol}(z, \delta) \neq 0$, onde:

$$
\begin{gathered}
\text { pol }=(1152+1920 \delta) z^{9}+\left(576 \delta^{2}-5664-11808 \delta\right) z^{8}+\left(11712+288 \delta^{3}-2880 \delta^{2}+30432 \delta\right) z^{7} \\
+\left(-42528 \delta+16 \delta^{4}-13788-936 \delta^{3}+6724 \delta^{2}\right) z^{6}+\left(36276 \delta-8508 \delta^{2}+10380+1148 \delta^{3}-48 \delta^{4}\right) z^{5} \\
+\left(6302 \delta^{2}-20040 \delta-680 \delta^{3}-5245+43 \delta^{4}\right) z^{4}+\left(180 \delta^{3}-2852 \delta^{2}+7312 \delta-6 \delta^{4}+1790\right) z^{3} \\
+\left(-398+791 \delta^{2}+2 \delta^{3}-7 \delta^{4}-1728 \delta\right) z^{2}+\left(-12 \delta^{3}-126 \delta^{2}+2 \delta^{4}+244 \delta+52\right) z \\
+2 \delta^{3}-3+9 \delta^{2}-16 \delta \neq 0 .
\end{gathered}
$$

Neste caso, a condição de transversalidade não será sempre diferente de zero, pelo menos no que diz respeito aos parâmetros $(\alpha, \beta, \mu)$, como podemos ver na figura 7.9. Conjecturamos que isto ocorre uma única vez para cada $\delta>0$ fixado. Resta, portanto, uma pergunta: qual seria a natureza dinâmica desses pontos? 


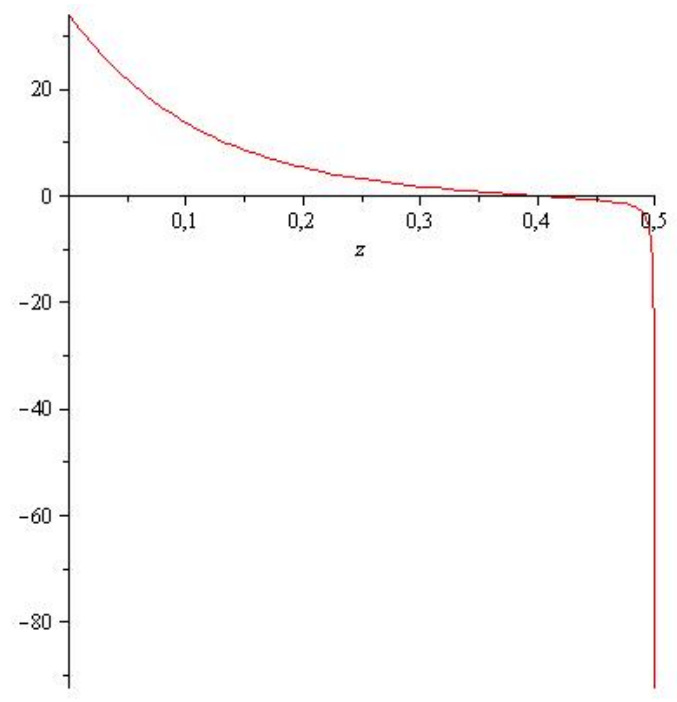

Figura 7.9: Fixamos $\delta=1$ e traçamos o gráfico de $C 3$. Note que ele se anula uma única vez.

Através de um calculo numérico, obtemos fortes indícios de que a aplicação:

$$
\mathcal{J}_{x, y, \alpha, \beta, \mu, \delta, \lambda}\left(f_{1}, f_{2}, \operatorname{Det}\left(\mathcal{J}_{x, y}\left(f_{1}, f_{2}\right)\right), \operatorname{Tr}\left(\mathcal{J}_{x, y}\left(f_{1}, f_{2}\right)\right), a 20\right)(x, y, \alpha, \beta, \mu, \delta, \lambda)
$$

é uma submersão. Assim, todos os pontos $(x, y)$ com parâmetros $(\alpha, \beta, \mu, \delta, \lambda)$ nos quais o Jacobiano é nilpotente, $a 20=0$ e as condições de não-degenerescência sejam satisfeitas serão bifurcação do tipo foco nilpotente ou elíptica nilpotente. O único detalhe é que alguns dentre estes pontos precisam dos parâmetros $(\delta, \lambda)$ para que a transversalidade desta bifurcação seja caracterizada.

Assim, os principais resultados deste capítulo podem ser resumidos nas seguintes proposições (usando o lema 7.1.2 para enuncia-los):

Proposição 7.7.4. Para os pontos $(x, y, \alpha, \beta, \mu, \delta, \lambda)$ candidatos a bifurcação de BogdanovTakens tais que $a 11 \neq 0 \neq a 20$, ocorre uma bifurcação do tipo Bogdanov-Takens.

Proposição 7.7.5. Existe um conjunto aberto e denso contido em $\Delta$, que chamaremos de ABT3 tal que, caso $(\delta, \lambda) \in A B T 3$ e o ponto $(x, y, \alpha, \beta, \mu, \delta, \lambda)$ seja candidato a bifurcação de Bogdanov-Takens, com a11 = 0 e cujas condições de não-degenerescência sejam satisfeitas, então ele é uma bifurcação de Bogdanov-Takens de codimensão três. 
Proposição 7.7.6. Existe um conjunto aberto e denso contido em $\Delta$, que chamaremos de AN tal que, caso $(\delta, \lambda) \in A N$ e o ponto $(x, y, \alpha, \beta, \mu, \delta, \lambda)$ seja candidato a bifurcação de Bogdanov-Takens, com a20 =0 e cujas condições de não-degenerescência sejam satisfeitas, então ele é um foco nilpotente ou um elíptico nilpotente.

Além disso, com fortes indícios numéricos, conjecturamos que:

- $A B T 3=\Delta$

- $A N=\Delta$

\subsection{Estudo da posição geométrica dos centros organi- zadores.}

As bifurcações transcrítica de codimensão três, Bogdanov-Takens de codimensão três e as nilpotentes degeneradas (foco e elíptica) são as bifurcações de codimensão mais alta estudadas neste trabalho. Dessa forma, elas são os chamados centros organizadores. Para o estudo do diagrama de bifurcação é importante termos uma ideia da posição geométrica destes centros organizadores. No capítulo 3 já fizemos este estudo para a bifurcação transcrítica de codimensão três. Nesta secção queremos fazer o mesmo para os outros centros organizadores.

Para isso, nosso principal objetivo é dividir $\Delta$ em regiões tais que, existam os mesmos centros organizadores em $\mathcal{W}$ para todos os $(\delta, \lambda)$ de uma determinada região.

Vamos começar analisando a bifurcação de Bogdanov-Takens de codimensão 3. Lembremos que esta bifurcação ocorre quando $a 11=0$ e que $a 11(x, z, \delta)=0 \mathrm{se}$, e somente se, $x=x_{B T 3}(z, \delta)$. Assim, notemos que, fixado $(z, \delta), \lambda_{B T 3}(z, \delta)=\frac{z}{x_{B T 3}(z, \delta)}$ é o único $\lambda$ tal que $a 11\left(x_{B T 3}, \lambda_{B T 3}, \delta\right)=0$. Em outras palavras, a família de pontos $\left(x_{B T 3}, \lambda_{B T 3}\right) \operatorname{com} 0<z \leq \frac{1}{2}$ é a família de soluções da equação $a 11(x, \lambda, \delta)=0$.

Assim, se quisermos estudar para quais $\lambda$ ocorrem uma bifurcação de Bogdanov-Takens 
de codimensão 3, devemos olhar para a imagem da função $\frac{z}{x_{B T 3}(z, \delta)}$. Mostraremos que essa função está bem definida e é não negativa:

Lema 7.8.1. $\frac{z}{x_{B T 3}(z, \delta)}$ está bem definido e é positivo para $0<z \leq \frac{1}{2}$. Além disso, admite extensão contínua para $z=0$.

Demonstração. Já mostramos que o denominador de $x_{B T 3}$ é não nulo. Assim, basta mostrar que o numerador também não é nulo e avaliar a função para algum $(z, \delta)$. Assim, tomando o numerador de $x_{B T 3}$, não é difícil provar (através do lema 7.1.1) que esta expressão é sempre não nula. Avaliando $x_{B T 3}$ em qualquer ponto, encontramos um valor positivo.

Por fim, o limite de $\frac{z}{x_{B T 3}(z, \delta)}$ quando $z$ tende a zero vale zero. Assim sendo, podemos estender a função para o compacto $\left[0, \frac{1}{2}\right]$.

Isso implica o seguinte corolário:

Corolário 7.8.2. Para cada $\delta_{0}$ fixo, existe um único $\lambda_{\delta_{0}, B T 3}$ tal que:

- caso $\lambda_{0} \leq \lambda_{\delta_{0}, B T 3}$ então existe pelo menos um ponto $\left(x, y, \alpha, \beta, \mu, \delta_{0}, \lambda_{0}\right)$ tal que $(x, y)$ com parâmetros $\left(\alpha, \beta, \mu, \delta_{0}, \lambda_{0}\right)$ é uma bifurcação de Bogdanov-Takens de codimensão três.

- caso $\lambda_{0}>\lambda_{\delta_{0}, B T 3}$ então não ocorre bifurcação de Bogdanov-Takens de codimensão três

Demonstração. Imediata do fato de que, funções contínuas em intervalos compactos têm máximo.

Assim, podemos passar a estudar a função $\frac{z}{x_{B T 3}(z, \delta)}$ quanto a sua injetividade:

Lema 7.8.3. Caso $(z, \delta)$ satisfaça a condição:

$$
\begin{gathered}
(288+384 \delta) z^{7}+(-1080-2280 \delta) z^{6}+\left(5040 \delta+1584+288 \delta^{2}\right) z^{5} \\
+\left(-1308-5214 \delta+2 \delta^{4}-1050 \delta^{2}-18 \delta^{3}\right) z^{4}+\left(3140 \delta+1080 \delta^{2}+696-4 \delta^{4}+120 \delta^{3}\right) z^{3} \\
+\left(-1206 \delta-240-611 \delta^{2}-96 \delta^{3}-3 \delta^{4}\right) z^{2}+\left(2 \delta^{4}+206 \delta^{2}+276 \delta+48+44 \delta^{3}\right) z \\
-4-\delta^{4}-29 \delta^{2}-28 \delta-10 \delta^{3} \neq 0
\end{gathered}
$$

então a derivada de $\frac{z}{x_{B T 3}}$ em z é não nula. 


\subsection{ESTUDO DA POSIÇÃO GEOMÉTRICA DOS CENTROS ORGANIZADORES. 147}

Antes de provarmos o lema, note que esta condição é a mesma que encontramos sobre a transversalidade do ponto de Bogdanov-Takens de codimensão três. Vale ressaltar que, assim como fizemos para a condição de transversalidade, vamos conjecturar, com indícios numéricos, que a derivada de $\frac{z}{x_{B T 3}}$ é sempre não nula.

Demonstração. Tomando a derivada de $\frac{z}{x_{B T 3}}$ em relação a $z$ obtemos uma expressão do tipo:

$$
\frac{p(z, \delta)+q(z, \delta) \sqrt{4 z^{2}-12 z \delta-4 z+\delta^{2}+6 \delta+1}}{h(z, \delta)}
$$

onde $p(z, \delta), q(z, \delta)$ e $h(z, \delta)$ são expressões polinomiais. Além disso, como $\frac{z}{x_{B T 3}}$ estava bemdefinida, a sua derivada também esta, o que significa que $h(z, \delta)$ não se anula no domínio válido (ou seja, $\delta>0$ e $0<z \leq 0$ ). Assim, o numerador é a parte relevante da expressão e está nas condições do lema 7.1.1. Assim, uma condição suficiente para que a derivada seja não nula é que a condição [i] não seja satisfeita, ou seja:

$$
\begin{gathered}
(288+384 \delta) z^{7}+(-1080-2280 \delta) z^{6}+\left(5040 \delta+1584+288 \delta^{2}\right) z^{5} \\
+\left(-1308-5214 \delta+2 \delta^{4}-1050 \delta^{2}-18 \delta^{3}\right) z^{4}+\left(3140 \delta+1080 \delta^{2}+696-4 \delta^{4}+120 \delta^{3}\right) z^{3} \\
+\left(-1206 \delta-240-611 \delta^{2}-96 \delta^{3}-3 \delta^{4}\right) z^{2}+\left(2 \delta^{4}+206 \delta^{2}+276 \delta+48+44 \delta^{3}\right) z \\
-4-\delta^{4}-29 \delta^{2}-28 \delta-10 \delta^{3} \neq 0
\end{gathered}
$$

Novamente, com fortes indícios numéricos, conjecturamos que essa derivada na verdade nunca se anule (ver figura 7.10). Isso implicaria que a função $\frac{z}{x_{B T 3}}$ é injetora para cada $\delta$ fixado. Assim sendo, para cada $\delta_{0}$ fixo e cada $\lambda_{0}$ na imagem fixo, existiria um único ponto $\left(x, y, \alpha, \beta, \mu, \delta_{0}, \lambda_{0}\right)$ tal que $(x, y)$ com parâmetros $\left(\alpha, \beta, \mu, \delta_{0}, \lambda_{0}\right)$ é uma bifurcação de Bogdanov-Takens de codimensão três.

Caso aceitemos a conjectura, podemos melhorar o último corolário. Como podemos mostrar que a derivada é sempre positiva, então $\lambda_{\delta, B T 3}$ é igual a $\frac{z}{x_{B T 3}}\left(\frac{1}{2}, \delta_{0}\right)=\frac{1}{2 \delta}$. Assim sendo teríamos que: abaixo da curva $x \delta=\frac{1}{2}$ ocorrem bifurcações de Bogdanov-Takens de codimensão três. Já acima desta mesma curva, não ocorrem tais bifurcações.

Vamos tratar agora do outro caso, ou seja, das bifurcações nilpotentes mais degeneradas: 




Figura 7.10: Gráfico de $\frac{z}{x_{B T 3}} \operatorname{com} \delta=\frac{1}{2}$. Note que a função é injetora e que a derivada da função nunca se anula.

Lema 7.8.4. $\frac{z}{x_{N 3}(z, \delta)}$ está bem definido e é positivo para $0<z<\frac{1}{2}$. Além disso, admite extensão contínua para $z=0$ e $z=\frac{1}{2}$.

Demonstração. Já mostramos que o denominador de $x_{N 3}$ é não nulo para $\delta>0$ e $0<z<\frac{1}{2}$. Agora, vamos mostrar que o numerador é não nulo. De fato, o numerador de $x_{N 3}$ vale:

$$
\begin{gathered}
-\delta^{2}(z-1)^{2}\left(2 \delta-12 z^{3}+4 z^{4}+10 \delta z^{2}-8 z \delta-4 z^{3} \delta+1-6 z+13 z^{2}+\delta^{2}+z^{2} \delta^{2}-2 z \delta^{2}+\right. \\
\left.+\sqrt{4 z^{2}-12 z \delta-4 z+\delta^{2}+6 \delta+1}(2 z-1+\delta)\right) .
\end{gathered}
$$

Assim, estamos nas condições do lema 7.1.1. A condição [i] equivale a:

$$
16 \delta^{2}(2 z-1)(z-1)^{4}=0
$$

o que não ocorre para $\delta>0$ e $0<z<\frac{1}{2}$. Assim sendo, o numerador está bem definido e a função $\frac{z}{x_{N 3}}$ está bem definida para $\delta>0$ e $0<z<\frac{1}{2}$. Resta mostrar que ela pode ser estendida. De fato, tomando o limite quando $z$ vai para zero e depois o limite quando $z$ vai para meio, obtemos que ambos os limites valem zero e, portanto, a função pode ser estendida.

Novamente isso implica em um corolário: 


\subsection{ESTUDO DA POSIÇÃO GEOMÉTRICA DOS CENTROS ORGANIZADORES. 149}

Corolário 7.8.5. Para cada $\delta_{0}$ fixo, existe um único $\lambda_{\delta_{0}, N 3}$ tal que:

- caso $\lambda_{0} \leq \lambda_{\delta_{0}, N 3}$ então existe pelo menos um ponto $\left(x, y, \alpha, \beta, \mu, \delta_{0}, \lambda_{0}\right)$ tal que $(x, y)$ com parâmetros $\left(\alpha, \beta, \mu, \delta_{0}, \lambda_{0}\right)$ é uma bifurcação nilpotente mais degenerada.

- caso $\lambda_{0}>\lambda_{\delta_{0}, N 3}$ então não ocorre bifurcação nilpotente mais degenerada.

Agora podemos estudar a função $\frac{z}{x_{N 3}}$ quanto a sua injetividade:

Lema 7.8.6. Caso $(z, \delta)$ satisfaça a condição:

$$
\begin{gathered}
(1152+1920 \delta) z^{9}+\left(576 \delta^{2}-5664-11808 \delta\right) z^{8}+\left(11712+288 \delta^{3}-2880 \delta^{2}+30432 \delta\right) z^{7} \\
+\left(-42528 \delta+16 \delta^{4}-13788-936 \delta^{3}+6724 \delta^{2}\right) z^{6}+\left(36276 \delta-8508 \delta^{2}+10380+1148 \delta^{3}-48 \delta^{4}\right) z^{5} \\
+\left(6302 \delta^{2}-20040 \delta-680 \delta^{3}-5245+43 \delta^{4}\right) z^{4}+\left(180 \delta^{3}-2852 \delta^{2}+7312 \delta-6 \delta^{4}+1790\right) z^{3} \\
+\left(-398+791 \delta^{2}+2 \delta^{3}-7 \delta^{4}-1728 \delta\right) z^{2}+\left(-12 \delta^{3}-126 \delta^{2}+2 \delta^{4}+244 \delta+52\right) z \\
+2 \delta^{3}-3+9 \delta^{2}-16 \delta \neq 0
\end{gathered}
$$

então a derivada de $\frac{z}{x_{N 3}}$ é não nula.

Demonstração. Essa demonstração é análoga à do lema 7.8.3.

Note que a condição do lema a mesma que encontramos sobre a transversalidade do foco e elíptica nilpotentes em relação aos parâmetros $(\alpha, \beta, \mu)$. Naquela ocasião mostramos que estes parâmetros podem não ser suficientes para que a bifurcação nilpotente seja transversal. Aqui, vamos mostrar que existe pelo menos um ponto em que a derivada de $\frac{z}{x_{N 3}}$ se anula. Embora não tenhamos uma demonstração formal, parece que a falta de transversalidade em relação aos parâmetros $(\alpha, \beta, \mu)$ está diretamente relacionada com a anulação da derivada de $\frac{z}{x_{N 3}}$.

Corolário 7.8.7. Para cada $\delta$ fixado, a função $\frac{z}{x_{N 3}}$ possui pelo menos um ponto no qual a derivada de $\frac{z}{x_{N 3}}$ em relação a z é nula. Além disso, isso só ocorre para um conjunto finito de pontos (com menos do que dez pontos).

Demonstração. Já mostramos que $\frac{z}{x_{N 3}}$ é positiva quando $0<z<\frac{1}{2}$ e $\delta>0$. Além disso, já mostramos que a extensão da função $\frac{z}{x_{N 3}}$ para $z=0$ e $z=\frac{1}{2}$ vale zero. Logo o máximo da função está no interior do intervalo e temos pelo menos um ponto cuja derivada de $\frac{z}{x_{N 3}}$ 


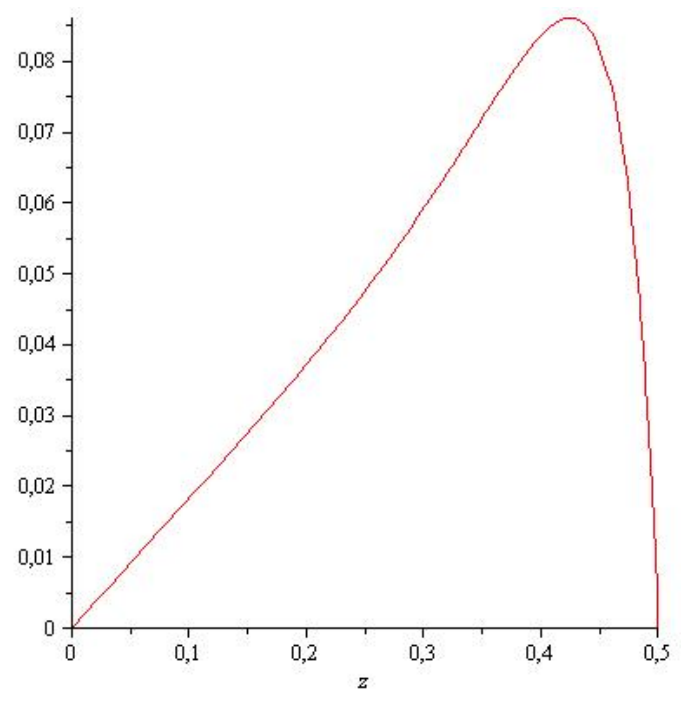

Figura 7.11: Gráfico de $\frac{z}{x N 3} \operatorname{com} \delta=\frac{1}{2}$. Note que em apenas um ponto a derivada se anula.

em relação a $z$ é nula. Além disso, como foi visto no lema anterior, para que a derivada se anule, $(z, \delta)$ tem que anular obrigatoriamente um polinômio com grau nove em $z$. Assim, tal polinômio só pode ter até nove soluções para cada $\delta$ fixo.

Como já foi adiantado, conjecturamos que, para cada $\delta>0$ fixado a derivada de $\frac{z}{x_{N 3}}$ em relação a $z$ se anula uma única vez para $0<z<\frac{1}{2}$ (ver figura 7.11). Isso significa que, para $\lambda<\lambda_{\delta_{0}, N 3}$ existem duas bifurcações do tipo nilpotente (foco ou elíptica).

Os principais resultados da secção podem ser resumidos nas duas seguintes proposições: (lembre-se que: se $f(x, y)$ é uma função contínua com $x \in K$ compacto, então $g(y)=$ $\max _{x \in K} f(x, y)$ é uma função contínua)

Proposição 7.8.8. O conjunto $\left(\delta, \lambda_{\delta, B T 3}\right)=\Omega_{1}$ é uma curva contínua que separa $\Delta$ em duas componentes conexas: uma acima da curva, outra abaixo. Na componente acima da curva, não ocorre nenhuma bifurcação de Bogdanov-Takens de codimensão três. Na componente abaixo da curva, ocorre um candidato a bifurcação de Bogdanov-Takens de codimensão três (não sabemos se com parâmetros válidos). Sobre $\Omega_{1}$ temos o mesmo comportamento da componente abaixo da curva. Ver ilustração 7.12 à esquerda.

Proposição 7.8.9. O conjunto $\left(\delta, \lambda_{\delta, N 3}\right)=\Omega_{2}$ é uma curva contínua que separa $\Delta$ em duas 
7.8. ESTUDO DA POSIÇÃO GEOMÉTRICA DOS CENTROS ORGANIZADORES. 151
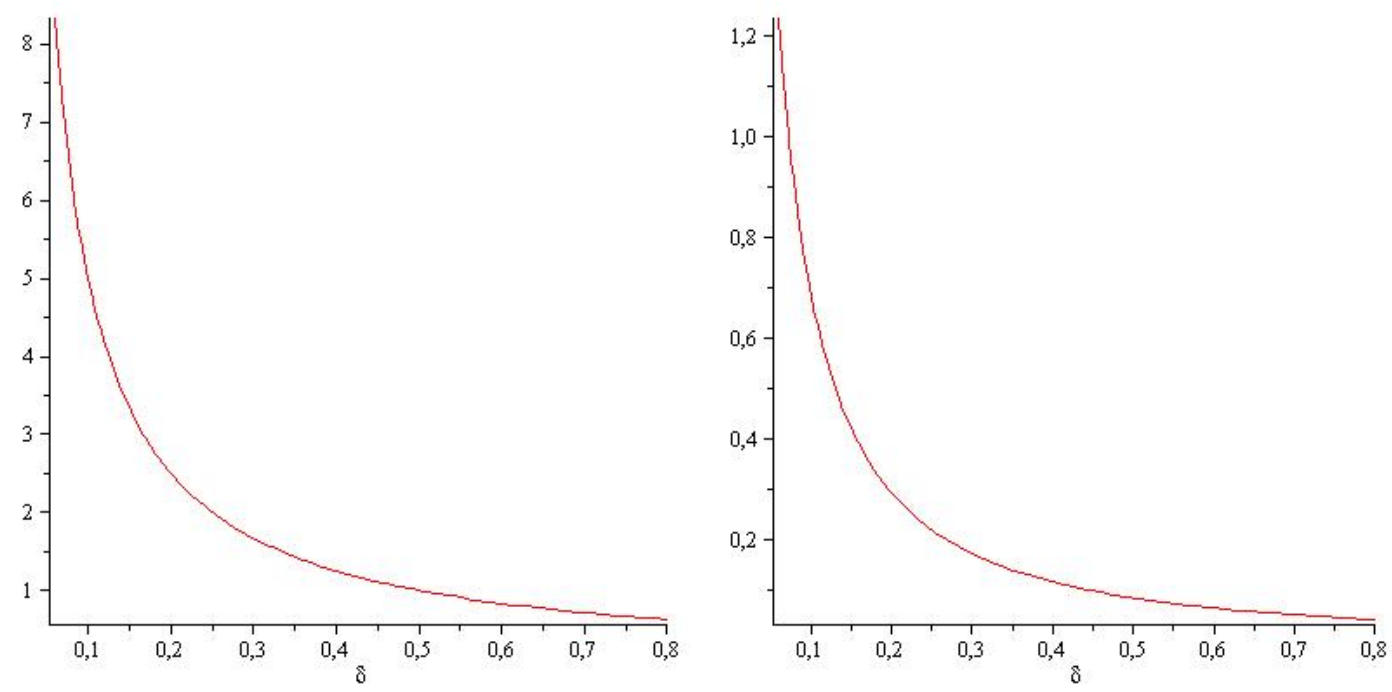

Figura 7.12: À esquerda a curva $\Omega_{1}$ e à direita a curva $\Omega_{2}$.

componentes conexas: uma acima da curva, outra abaixo. Na componente acima da curva, não ocorre nenhuma bifurcação do tipo nilpotente. Na componente abaixo da curva, ocorre pelo menos dois candidatos a bifurcação nilpotente, sendo que este número pode chegar a dez (não sabemos se com parâmetros válidos). Sobre $\Omega_{2}$ temos a ocorrência de um único candidato a bifurcação do tipo nilpotente (não sabemos se com parâmetros válidos). Ver ilustração 7.12 à direita.

Com base em fortes indícios numéricos podemos conjecturar os seguintes refinamentos:

- $\Omega_{1}=\left(\delta, \frac{1}{2 \delta}\right)$

- Caso estejamos na componente abaixo de $\Omega_{1}$, teremos exatamente uma única bifurcação de Bogdanov-Takens de codimensão três.

- Caso estejamos na componente abaixo de $\Omega_{2}$, teremos exatamente duas bifurcações do tipo nilpotente.

Anotamos que a curva $\mathcal{C}$ do trabalho [1] coincide com a curva $\Omega_{2}$ do presente trabalho. 


\subsection{Estudo sobre a validade dos parâmetros onde ocor- rem bifurcações de Bogdanov-Takens mais degen- eradas}

Na primeira secção achamos uma curva de candidatos a bifurcação de Bogdanov-Takens e conseguimos restringir algebricamente o domínio da curva de forma que o parâmetro $\mu$ seja sempre maior ou igual a zero. Entretanto,para os parâmetros $\alpha$ e $\beta$ conseguimos informações implícitas sobre a validade da curva, sem chegar a restringir o domínio da curva. Dessa forma, em todos os estudos precedentes, não impomos restrições sobre $\alpha$ e $\beta$. Isso significa que, as bifurcações de Bogdanov-Takens mais degeneradas encontradas podem não ser vigentes para parâmetros válidos.

Vamos estudar quando ocorre uma bifurcação de Bogdanov-Takens mais degenerada para $\alpha \geq 0$ e $\beta>0$, deixando bem claro qual restrição estamos considerando em cada momento. O intuito disso é separar as restrições sobre $\alpha$ e $\beta$ de forma a poder usa-las em diferentes modelos, já que, tanto em $\mathcal{W}$ quanto em $\mathcal{W}_{1}, \alpha$ é maior ou igual a zero, mas em relação a $\beta$ temos diferença entre ambos os domínios.

Antes de começarmos, vamos apresentar a estratégia: Quando estudamos as bifurcações mais degeneradas e as restrições que $\alpha \geq 0$ e $\beta>0$ impõem na curva de candidatos, procedemos da seguinte forma:

- Avaliamos as devidas funções $\left(A, \beta_{t r}, a 20\right.$ e a11) sobre a curva $\left(z, y_{B T 1}, A, \beta_{t r}, M, \delta, \lambda\right)$.

- Fizemos a mudança de coordenadas $z=x \lambda$.

- Resolvemos a equação em função de $x$ obtendo $x_{i}(z, \delta)$ onde $i$ pode ser $A, B, N 3$ ou BT3.

- Recuperamos informações sobre $(\delta, \lambda)$ através da função $\frac{z}{x_{i}(z, \delta)}$.

Para recuperar as informações, tomávamos $\left(\delta_{0}, \lambda_{0}\right) \in \Delta$, fixávamos $\delta=\delta_{0}$ e tomávamos o inverso da função $\frac{z}{x_{i}\left(z, \delta_{0}\right)}$ em relação a $\lambda_{0}$. Agora, vamos comparar os gráficos $\frac{z}{x_{i}\left(z, \delta_{0}\right)}$ de forma 
a saber se o gráfico relativo a $\lambda_{B T 3}$ e $\lambda_{N 3}$ estão nas regiões válidas dos gráficos de $\lambda_{A}$ e $\lambda_{B}$. Essa estratégia já foi usada para demonstrar o lema 7.2.6.

Assim, vamos começar tratando das bifurcações de Bogdanov-Takens de codimensão três:

Lema 7.9.1. Fixado $(\delta, \lambda) \in \Delta$, seja $(x, y, \alpha, \beta, \mu, \delta, \lambda)$ contido na curva $\left(x, y_{B T 1}, A, \beta_{t r}, M, \delta, \lambda\right)$ com $0<x \lambda \leq \frac{1}{2}$ tal que seja um ponto de bifurcação de Bogdanov-Takens de codimensão três. Neste caso, $\alpha \geq 0$.

Lembremos que, os únicos candidatos a bifurcação de Bogdanov-Takens de codimensão três que podem ter os parâmetros válidos, estão na curva $\left(x, y_{B T 1}, A, \beta_{t r}, M, \delta, \lambda\right)$ com $0<$ $x \lambda \leq \frac{1}{2}$ e portanto essa hipótese não representa nenhuma perda de generalidade para o problema inicial, que é estabelecer as bifurcações de Bogdanov-Takens de codimensão três com parâmetros (todos) válidos.

Demonstração. Para essa demonstração vamos proceder da seguinte maneira:

- Vamos mostrar que $x_{A} \neq x_{B T 3}$ para todo $(z, \delta)$ válido (lembremos que $z \neq \frac{1}{2}$ para que $x_{A}$ esteja bem definido). Assim sendo, mostramos que a função $A\left(x_{B T 3}, y_{B T 1}, \beta_{t r}, \delta, z\right)$ nunca é igual a zero, já que isso ocorre se, e somente se, $x=x_{A}$.

- Como as funções $A$ e $x_{B T 3}$ são contínuas, a função $A\left(x_{B T 3}, y_{B T 1}, \beta_{t r}, \delta, z\right)$ é contínua e, portanto, permanece sempre com o mesmo sinal.

- Avaliamos a função $A\left(x_{B T 3}, y_{B T 1}, \beta_{t r}, \delta, z\right)$ em qualquer ponto e obtemos que ela é positiva.

Para mostrar que $x_{A} \neq x_{B T 3}$ para todo $(z, \delta)$ válido, comecemos lembando que $x_{A}$ e $x_{B T 3}$ estão bem definidos para $0<z<\frac{1}{2}$. Dessa forma, a função $F_{c}=x_{B T 3}-x_{A}$ está bem definida para todo $(z, \delta)$ válido. Assim, como estamos interessados em saber se está expressão é igual a zero ou não, podemos nos preocupar apenas com o numerador de $F_{c}$ que é igual a:

$$
2 \delta^{2}\left(p(z, \delta)+q(z, \delta) \sqrt{4 z^{2}-4 z-12 z \delta+1+6 \delta+\delta^{2}}\right)
$$


onde:

$$
\begin{gathered}
p(z, \delta)=(-1+z)\left(8 z^{4}-8 z^{3}-40 z^{3} \delta+32 z^{2} \delta+58 z^{2} \delta^{2}+2 z^{2}\right. \\
\left.-8 z \delta-38 z \delta^{2}-24 z \delta^{3}+2 \delta^{4}+\delta+10 \delta^{3}+7 \delta^{2}\right) \\
q(z, \delta)=(-1+z)\left(4 z^{3}-2 z^{2}-14 z^{2} \delta+12 z \delta^{2}+6 z \delta-\delta-2 \delta^{3}-4 \delta^{2}\right)
\end{gathered}
$$

Assim, estamos nas condições do lema 7.1.1 e a condição [i] equivale a:

$$
8 \delta^{4}(-1+z)^{2}\left(4 z^{3}+2 z^{2} \delta^{2}-6 z^{2} \delta+2 z \delta-2 z \delta^{2}+\delta^{2}\right)=0
$$

Como $z<\frac{1}{2}$ e $\delta>0$, temos duas possibilidades de solução:

- $\delta=d 1=\frac{\left(3 z-1+\sqrt{17 z^{2}-10 z+1-8 z^{3}}\right) z}{2 z^{2}-2 z+1}$

- $\delta=d 2=\frac{\left(3 z-1-\sqrt{17 z^{2}-10 z+1-8 z^{3}}\right) z}{2 z^{2}-2 z+1}$

Não é difícil notar que $d 1$ e $d 2$ são negativos ou complexos para $0<z \leq \frac{1}{2}$, e portanto não são válidos.

Assim, resta analisar apenas a última parte da demonstração. Avaliando $A\left(x_{B T 3}, y_{B T 1}, \beta_{t r}, \delta, z\right)$ em $(z, \delta)=\left(\frac{1}{4}, 1\right)$ obtemos aproximadamente $0.104>0$. Logo essa função é sempre positiva.

Agora vamos considerar as restrições sobre $\beta$.

Lema 7.9.2. Fixado $\delta>0$, seja $(x, y, \alpha, \beta, \mu, \delta, \lambda)$ contido na curva $\left(x, y_{B T 1}, A, \beta_{t r}, M, \delta, \lambda\right)$ com $0<x \lambda \leq \frac{1}{2}$ um ponto de bifurcação de Bogdanov-Takens de codimensão três. Então, existe $\lambda_{\delta, B T 3, \text { max }}$ tal que, caso $\lambda<\lambda_{\delta, B T 3, \text { max }}$, então $\beta>0$ e, caso $\lambda>\lambda_{\delta, B T 3, \text { max }}$, então $\beta<0$.

Além disso, sempre que $x \lambda \geq \frac{1}{3}$, então $\beta \leq 0$.

Após demonstrar o lema, faremos conjecturas sobre $\lambda=\lambda_{\delta, B T 3, \max }$.

Demonstração. Vamos começar a demonstração exatamente na mesma forma que na anterior: seja $F_{c}=x_{B T 3}-x_{B}$. Sabemos que esta expressão está bem definida para $(z, \delta)$ válidos. 
Como estamos interessados em saber quando $F_{c}$ se anula, podemos considerar apenas o numerador de $F_{c}$ que é igual a:

$$
4 \delta^{2}\left(p(z, \delta)+q(z, \delta) \sqrt{4 z^{2}-4 z-12 z \delta+1+6 \delta+\delta^{2}}\right)
$$

onde:

$$
\begin{gathered}
p(z, \delta)=(-1+z)\left(12 z^{4}-16 z^{3}-56 z^{3} \delta+58 z^{2} \delta+7 z^{2}+73 z^{2} \delta^{2}\right. \\
\left.-58 z \delta^{2}-19 z \delta-z-26 z \delta^{3}+2 \delta^{4}+12 \delta^{3}+2 \delta+12 \delta^{2}\right) \\
q(z, \delta)=(-1+z)\left(6 z^{3}-19 z^{2} \delta-5 z^{2}+12 z \delta+14 z \delta^{2}+z-6 \delta^{2}-2 \delta^{3}-2 \delta\right)
\end{gathered}
$$

Estamos nas condições do lema 7.1.1. Assim, para estudar quando $F_{c}$ se anula, comecemos estudando a condição [i]:

$$
8 \delta^{4}(z-1)^{2}\left(9 z^{3}+3 z^{3} \delta-16 \delta z^{2}-6 z^{2}+z^{2} \delta^{2}+11 z \delta+z-2 \delta\right)=0 .
$$

Como $z<1$ e $\delta>0$ temos três possibilidades:

- $z=\frac{1}{2}$

- $\delta=d 1=1 / 2 \frac{\left(-z^{2}+5 z-2+\sqrt{z^{4}-14 z^{3}+29 z^{2}-20 z+4}\right)(-1+3 z)}{z^{2}}$

- $\delta=d 2=1 / 2 \frac{\left(-z^{2}+5 z-2-\sqrt{z^{4}-14 z^{3}+29 z^{2}-20 z+4}\right)(-1+3 z)}{z^{2}}$

A primeira opção pode ser facilmente descartada já que:

$$
F_{c}\left(\frac{1}{2}, \delta\right)=-\delta \neq 0
$$

Assim, restam $\delta=d 1$ e $\delta=d 2$. Como o domínio em $z$ de $d 1$ e $d 2$ é $\left.] 0, \frac{1}{2}\right]$, não é difícil ver que, $d 1$ e $d 2$ são estritamente positivas e reais se, e somente se, $z \in] 0, \frac{1}{3}[$. Assim, sempre devemos estar neste intervalo. Vamos prosseguir o estudo para a condição [ii].

Agora, não é difícil provar que:

- $p(z, d 1)<0$ para $z \in] 0, \frac{1}{3}[$,

- $p(z, d 2)<0$ para $z \in] 0, \frac{1}{3}[$, 


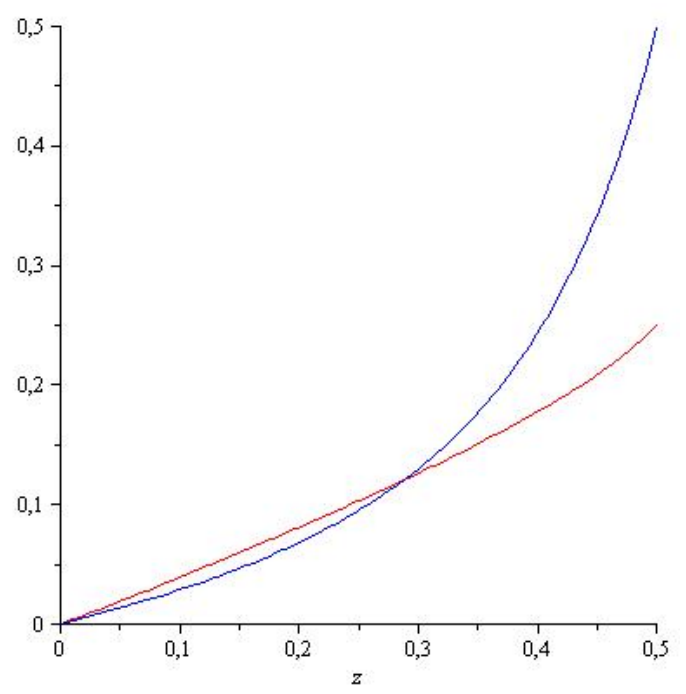

Figura 7.13: Em azul o gráfico $\frac{z}{x_{B T 3}}$ e em vermelho o gráfico $\frac{z}{x_{B}}$. Para o cálculo deste gráfico, tomamos $\delta=1$.

- $q(z, d 1)<0$ para $z \in] 0, \frac{1}{3}[$,

- $q(z, d 2)>0$ para $z \in] 0, \frac{1}{3}[$.

Para mostrar isso basta tomar cada uma dessas funções, notar que elas satisfazem o lema 7.1.1, mostrar que elas não se anulam para $z \in] 0, \frac{1}{3}$ [ já que a condição [i] não é verificada e calculá-las em qualquer ponto de $z \in] 0, \frac{1}{3}[$. Feito isso, notamos que $d 1$ não satisfaz a condição [ii] e portanto não anula $F_{c}$, mas que $d 2$ por sua vez satisfaz essa condição, logo anula $F_{c}$.

Agora, vamos estudar esta raiz. Primeiramente, não é difícil ver que d2 é uma função injetora de ]0, $\frac{1}{3}[$ em ]0, $\infty$ ( tome a derivada, note que ela satisfaz as condições do lema 7.1.1 e mostre que ela não se anula neste intervalo já que a condição [i] não é satisfeita). Dessa forma, ela pode ser invertida, de forma que, para cada $\delta$ fixado, exista um único $z \in] 0, \frac{1}{3}[$ tal que $x_{B}=x_{B T 3}$. Assim sendo, para cada $\delta$ fixado, os gráficos $\frac{z}{x_{B}}$ e $\frac{z}{x_{B T 3}}$ se cruzam uma única vez para algum $z \in] 0, \frac{1}{3}\left[\right.$. Chamemos esse $z$ de $z_{\delta, B-B T 3}$. Ver figura 7.13.

Dessa forma, podemos dividir o domínio ]0, $\left.\frac{1}{2}\right]$ em duas componentes a menos de um ponto: ] $\left.0, z_{\delta, B-B T 3}[\mathrm{e}] z_{\delta, B-B T 3}, \frac{1}{2}\right]$. Em cada uma dessas componentes o gráfico de $\frac{z}{x_{B T 3}}$ estará to- 
talmente acima do gráfico de $\frac{z}{x_{B}}$ ou totalmente abaixo. Lembremos que, $\beta$ é maior que zero, quando estivermos abaixo do gráfico. Com base da figura 7.13 e na continuidade das funções $\frac{z}{x_{B T 3}}$ e $\frac{z}{x_{B}}$ em relação a $z$ e $\delta$, chegamos à conclusão de que na componente $] 0, z_{\delta, B-B T 3}[\mathrm{o}$ gráfico de $\frac{z}{x_{B T 3}}$ está abaixo de $\frac{z}{x_{B}}$ e na componente $\left.] z_{\delta, B-B T 3}, \frac{1}{2}\right]$ está acima de $\frac{z}{x_{B}}$.

Como $\frac{z}{x_{B T 3}}$ é uma função contínua, ela possui um máximo em $\left[0, z_{\delta, B-B T 3}\right]$ que chamaremos de $\lambda_{\delta, B T 3, \max }$. Assim, caso $\lambda>\lambda_{\delta, B T 3, \max }$, não temos bifurcação de Bogdanov-Takens de codimensão três com $\beta>0$.

Por fim, caso $x \lambda \geq \frac{1}{3}$, então isso significa que estamos na componente $\left.] z_{\delta, B-B T 3}, \frac{1}{2}\right]$ e, portanto, $\beta<0$.

Dessa forma, se aceitarmos a conjectura de que $\frac{z}{x_{B T 3}}$ é uma função injetora para $\delta$ fixo, chegamos a seguinte conclusão:

- $\lambda_{\delta, B T 3, \max }=\frac{z}{x_{B T 3}}\left(z_{\delta, B-B T 3}, \delta\right)$.

- Não existe bifurcação de Bogdanov-Takens para $\lambda \geq \lambda_{\delta, B T 3, \max }$.

Este último lema é interessante tanto por mostrar que existe um $\lambda$ único de transição quanto por nos fornecer uma interpretação geométrica: caso $x \lambda \geq \frac{1}{3}$, então a bifurcação de Bogdanov-Takens de codimensão três não ocorre para parâmetros válidos (em $\mathcal{W}_{1}$ ).

Vamos agora tratar do caso das bifurcações nilpotentes: foco e elíptica.

Lema 7.9.3. Fixado $(\delta, \lambda) \in \Delta$, seja $(x, y, \alpha, \beta, \mu, \delta, \lambda)$ contido na curva $\left(x, y_{B T 1}, A, \beta_{t r}, M, \delta\right.$, ג) com $0<x \lambda<\frac{1}{2}$ tal que seja um ponto de bifurcação nilpotente. Neste caso, $\beta>0$.

Demonstração. Para essa demonstração vamos proceder da mesma maneira que nos lemas anteriores:

- Vamos mostrar que $x_{B} \neq x_{N 3}$ para todo $(z, \delta)$ válido. Assim sendo, verificaremos que a função $\beta_{t r}\left(x_{B T 3}, y_{B T 1}, \delta, z\right)$ nunca é igual a zero, já que isso ocorre se, e somente se, $x=x_{B}$. 
- Como as funções $\beta_{t r}$ e $x_{N 3}$ são contínuas, a função $\beta_{t r}\left(x_{B T 3}, y_{B T 1}, \delta, z\right)$ é contínua e, portanto, permanece sempre com o mesmo sinal.

- Avaliamos a função $\beta_{t r}\left(x_{B T 3}, y_{B T 1}, \delta, z\right)=\beta_{t r}(z, \delta)$ em qualquer ponto e obtemos que ela é positiva.

Para mostrar que $x_{B} \neq x_{N 3}$ para todo $(z, \delta)$ válido, comecemos lembando que $x_{B}$ e $x_{N 3}$ estão bem definidos para $0<z<\frac{1}{2}$ e que, para $z=\frac{1}{2}$ não temos candidato a bifurcação nilpotente. Dessa forma, a função $F_{c}=x_{N 3}-x_{B}$ está bem definida para todo $(z, \delta)$ válido com $z \neq \frac{1}{2}$. Assim, como estamos interessados em saber se está expressão é igual a zero ou não, podemos nos preocupar apenas com o numerador de $F_{c}$ que é igual a:

$$
2 \delta^{2}\left(p(z, \delta)+q(z, \delta) \sqrt{4 z^{2}-4 z-12 z \delta+1+6 \delta+\delta^{2}}\right)
$$

onde:

$$
\begin{gathered}
p(z, \delta)=(-1+z)\left(24 z^{5}-44 z^{4}-100 z^{4} \delta+86 z^{3} \delta^{2}+140 z^{3} \delta+30 z^{3}\right. \\
-69 z^{2} \delta+31 z^{2} \delta^{3}-9 z^{2}-81 z^{2} \delta^{2}+14 z \delta+27 z \delta^{2}+z-24 \delta^{4} z \\
\left.-34 z \delta^{3}-\delta+7 \delta^{3}+12 \delta^{4}-4 \delta^{2}+2 \delta^{5}\right) \\
q(z, \delta)=(-1+z)\left(12 z^{4}-16 z^{3}-32 z^{3} \delta+7 z^{2} \delta^{2}+30 z^{2} \delta+7 z^{2}\right. \\
\left.-2 z \delta^{2}-9 z \delta+12 z \delta^{3}-z-2 \delta^{4}-6 \delta^{3}+\delta+\delta^{2}\right)
\end{gathered}
$$

Assim, estamos nas condições do lema 7.1 .1 e a condição [i] equivale a:

$$
8 \delta^{5}(z-1)^{2}(z \delta-\delta+z)\left(36 z^{3}+18 \delta z^{2}-48 z^{2}-z \delta^{2}-20 z \delta+21 z+\delta^{2}+6 \delta-3\right)=0
$$

Como $z<\frac{1}{2}$ e $\delta>0$, temos três possibilidades de solução:

$$
\begin{aligned}
& -\delta=d 1=\frac{9 z^{2}+3-10 z+\sqrt{12+223 z^{2}-84 z+117 z^{4}-264 z^{3}}}{z-1} \\
& \text { - } \delta=d 2=\frac{9 z^{2}+3-10 z-\sqrt{12+223 z^{2}-84 z+117 z^{4}-264 z^{3}}}{z-1} \\
& \text { - } \delta=d 3=-\frac{z}{z-1}
\end{aligned}
$$

Não é difícil notar que $d 1$ é negativo $0<z<\frac{1}{2}$ e portanto não é válido. Além disso, é fácil mostrar que $d 2$ é positivo se, e somente se, $0<z<\frac{1}{3}$ (já que estamos restritos a $\left.\left.z \in\right] 0, \frac{1}{2}\right]$ ). Dessa forma, precisamos analisar a condição [ii] em relação a $d 2$ e $d 3$, sendo que este primeiro apenas para $0<z<\frac{1}{3}$. 
Agora, não é difícil provar que:

- $p(z, d 2)<0$ para $z \in] 0, \frac{1}{3}[$,

- $p(z, d 3)<0$ para $z \in] 0, \frac{1}{2}[$,

- $q(z, d 2)<0$ para $z \in] 0, \frac{1}{3}[$,

- $q(z, d 3)<0$ para $z \in] 0, \frac{1}{2}[$.

Para mostrar isso basta tomar cada uma dessas funções, notar que elas satisfazem o lema 7.1.1, mostrar que elas não se anulam para $z \in] 0, \frac{1}{3}[$ ou $z \in] 0, \frac{1}{2}[$ (dependendo do caso) já que a condição [i] não é verificada e calculá-las em qualquer ponto de $z \in] 0, \frac{1}{3}[$ ou $z \in] 0, \frac{1}{2}[$. Feito isso, notamos que $d 2$ e $d 3$ não satisfazem a condição [ii] e portanto não anulam $F_{c}$.

Assim, resta avaliar a função $\beta_{t r}(z, \delta)$. Calculando no ponto $(z, \delta)=\left(\frac{1}{4}, 1\right)$ obtemos aproximadamente $0.664>0$. Dessa forma, chegamos a conclusão que $\beta_{t r}(z, \delta)>0$.

Assim, resta tratar da última restrição: $\alpha \geq 0$ :

Lema 7.9.4. Fixado $\delta>0$, seja $(x, y, \alpha, \beta, \mu, \delta, \lambda)$ contido na curva $\left(x, y_{B T 1}, A, \beta_{t r}, M, \delta, \lambda\right)$ com $0<x \lambda \leq \frac{1}{2}$ um ponto de bifurcação nilpotente degenerada. Então, existe $z_{\delta, A-N 3}$ tal que, caso $x \lambda<z_{\delta, A-N 3}$ então $\alpha<0$ e, caso $x \lambda \geq z_{\delta, A-N 3}$ então $\alpha \geq 0$. Além disso, sempre que $x \lambda \leq \frac{1}{4}$, então $\alpha<0$, pois $\frac{1}{4}<z_{\delta, A-N 3}<\frac{1}{3}$.

Demonstração. Vamos proceder da mesma forma que nas últimas demonstrações. Começamos definindo a função $F_{c}=x_{N 3}-x_{A}$. Tal função está bem definida para $0<z<\frac{1}{2}$ e devemos lembrar que, caso $z=\frac{1}{2}$, não temos bifurcações nilpotentes. Assim, para saber quando essa expressão é igual a zero, precisamos analisar apenas o numerador:

$$
2 \delta^{2}\left(p(z, \delta)+q(z, \delta) \sqrt{4 z^{2}-4 z-12 z \delta+1+6 \delta+\delta^{2}}\right)
$$


onde:

$$
\begin{gathered}
p(z, \delta)=(-1+z)\left(8 z^{5}-32 z^{4} \delta-12 z^{4}+32 z^{3} \delta+6 z^{3}+22 z^{3} \delta^{2}\right. \\
+20 z^{2} \delta^{3}-8 z^{2} \delta-z^{2}-3 z^{2} \delta^{2}-12 \delta^{4} z-6 z \delta^{2}-24 z \delta^{3} \\
\left.+6 \delta^{4}+6 \delta^{3}+\delta^{5}+\delta^{2}\right) \\
q(z, \delta)=(-1+z)\left(4 z^{4}-4 z^{3}-10 z^{3} \delta+5 z^{2} \delta+z^{2}+6 z \delta^{3}+4 z \delta^{2}-\delta^{2}-3 \delta^{3}-\delta^{4}\right)
\end{gathered}
$$

Assim, estamos nas condições do lema 7.1.1 e a condição [i] equivale a:

$$
4 \delta^{5}(2 z-1)(-1+z)^{2}\left(4 z^{2}+3 z \delta-z-\delta\right)(z \delta+z-\delta)=0
$$

Como $z<\frac{1}{2}$ e $\delta>0$, temos duas possibilidades de solução válidas:

- $\delta=d 1=\frac{z}{1-z}$

- $\delta=d 2=-\frac{z(4 z-1)}{3 z-1}$

Antes de continuarmos note que $d 2$ não está bem definido para $z=\frac{1}{3}$. No entanto, ao substituirmos em $4 z^{2}+3 z \delta-z-\delta$, $z$ por $\frac{1}{3}$ obtemos $\frac{1}{9} \neq 0$ e portanto não estamos perdendo informação ao resolver essa equação em função de $\delta$.

Assim, devemos passar ao estudo da condição [ii]. Começando por $\delta=d 1$ não é difícil mostrar que:

- $p(z, d 1)>0$ para $] 0, \frac{2-\sqrt{2}}{2}[$

- $q(z, d 1)>0$ para $] 0, \frac{2-\sqrt{2}}{2}[$

- $p(z, d 1)<0$ para $] \frac{2-\sqrt{2}}{2}, \frac{1}{2}[$

- $q(z, d 1)<0$ para $] \frac{2-\sqrt{2}}{2}, \frac{1}{2}[$

Dessa forma, fora de $z=\frac{2-\sqrt{2}}{2}, d 1$ não é solução. No entanto, devemos notar que:

$$
d 1\left(\frac{2-\sqrt{2}}{2}\right)=-1 / 2(-2+\sqrt{2}) \sqrt{2}=d 2\left(\frac{2-\sqrt{2}}{2}\right)
$$

$\operatorname{logo}$, tratando o caso $\delta=d 2$ já estamos tratando deste caso particular. Comecemos notando que $d 2$ é positivo e bem definido se, e somente se $\frac{1}{4}<z<\frac{1}{3}$. Assim, não é difícil mostrar que: 


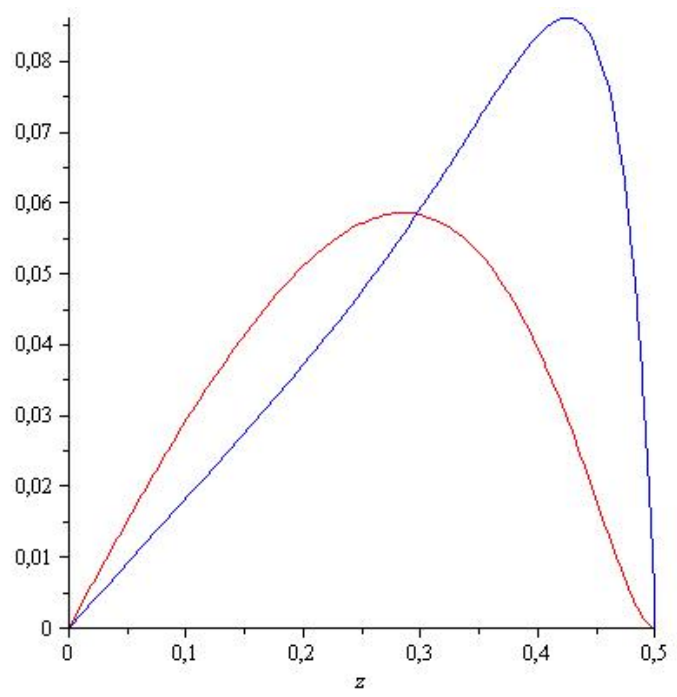

Figura 7.14: Em azul o gráfico $\frac{z}{x_{N 3}}$ e em vermelho o gráfico $\frac{z}{x_{A}}$. Para o cálculo deste gráfico, tomamos $\delta=\frac{1}{2}$.

- $p(z, d 2)>0$ para $] \frac{1}{4}, \frac{2-\sqrt{2}}{2}[$

- $q(z, d 2)<0$ para $] \frac{1}{4}, \frac{2-\sqrt{2}}{2}[$

- $p(z, d 2)<0$ para $] \frac{2-\sqrt{2}}{2}, \frac{1}{3}[$

- $q(z, d 2)>0$ para $] \frac{2-\sqrt{2}}{2}, \frac{1}{3}[$

Dessa forma, chegamos a conclusão que $\delta=d 2$ é solução para todo $z \in] \frac{1}{4}, \frac{1}{3}[$. Além disso, não é difícil mostrar que $d 2(z)$ leva injetivamente $] \frac{1}{4}, \frac{1}{3}[$ em $] 0, \infty[$ (mostre que a derivada é sempre positiva e os limites são zero e $+\infty$ ). Assim, podemos inverter $d 2$ de forma que para todo $\delta>0$ exista um único $z$ onde $x_{A}=x_{N 3}$. Chamaremos esse $z$ de $z_{\delta, A-N 3}$.

Dessa forma, para cada $\delta>0$ fixo, os gráficos de $\frac{z}{x_{A}}$ e $\frac{z}{x_{N} 3}$ se cruzam apenas uma vez em $z=z_{\delta, A-N 3}$. Dessa forma, podemos dividir o domínio ]0, $\left.\frac{1}{2}\right]$ em duas componentes a menos de um ponto: $\left.] 0, z_{\delta, A-N 3}[\mathrm{e}] z_{\delta, A-N 3}, \frac{1}{2}\right]$. Em cada uma dessas componentes o gráfico de $\frac{z}{x_{N 3}}$ estará totalmente acima do gráfico de $\frac{z}{x_{A}}$ ou totalmente abaixo. Lembremos que $\alpha$ é maior do que zero quando estivermos acima do gráfico. Com base na figura 7.14 e na continuidade das funções $\frac{z}{x_{N 3}}$ e $\frac{z}{x_{A}}$ em relação a $z$ e $\delta$, chegamos à conclusão de que na 
componente $] 0, z_{\delta, A-N 3}\left[\right.$ o gráfico de $\frac{z}{x_{N 3}}$ está abaixo de $\frac{z}{x_{A}}$ e na componente $\left.] z_{\delta, A-N 3}, \frac{1}{2}\right]$ o gráfico está acima de $\frac{z}{x_{A}}$.

Assim, fixado $\delta>0$, a bifurcação só ocorre para $\alpha \geq 0$ caso $z \geq z_{\delta, A-N 3}$.

Podemos completar o lema anterior:

Lema 7.9.5. Existe uma curva $\Omega_{3}$ de $\Delta$ que está totalmente abaixo de $\Omega_{2}$. Essa curva divide a componente abaixo de $\Omega_{2}$ em duas componentes com diferenças quanto ao diagrama de bifurcações. Para um conjunto aberto e denso de $\Delta$, temos:

- Se $(\delta, \lambda)$ estiver abaixo de $\Omega_{2}$ e acima de $\Omega_{3}$ ocorrem pelo menos duas bifurcações nilpotentes degeneradas sendo que todas com parâmetros válidos.

- Se $(\delta, \lambda)$ estiver abaixo de $\Omega_{3}$ ocorrem pelo menos duas bifurcações nilpotentes degeneradas, sendo que, pelo menos uma dentre essas bifurcações ocorre para parâmetros não-válidos.

Demonstração. Seja agora $\lambda_{\delta_{0}, A-N 3, \max }$ o supremo da função $\frac{z}{x_{N 3}}$ para $0<z<z_{\delta, A-N 3}$. Então, claramente se $\lambda<\lambda_{\delta_{0}, A-N 3, \max }$, existirá pelo menos uma bifurcação nilpotente que ocorre fora dos parâmetros válidos. Já se $\lambda>\lambda_{\delta_{0}, A-N 3, \max }$ então todas as bifurcações nilpotentes (se existirem) ocorrerão com $\alpha \geq 0$. Assim sendo, podemos definir $\Omega_{3}=$ $\left(\delta, \lambda_{\delta_{0}, A-N 3, \max }\right)$ curva em $\Delta$. Esta é a curva procurada.

Agora, podemos apresentar dois corolários:

Corolário 7.9.6. Existe $\delta_{0}>0$ e $\lambda_{0}>0$ tal que, para $\lambda<\lambda_{0}$ existe apenas um candidato a bifurcação nilpotente, enquanto para $\lambda_{\delta, N 3}>\lambda \geq \lambda_{0}$ existem dois candidatos a bifurcação nilpotente. Além disso, caso $\lambda>\lambda_{\delta, N 3}$ não ocorrem mais bifurcações nilpotentes.

Demonstração. Corolário imediato dos dois lemas anteriores e da figura 7.14. Como já vimos em secções anteriores $\lambda>\lambda_{\delta, N 3}$ não ocorrem mais bifurcações nilpotentes.

Conjecturamos que a conclusão deste corolário é verdadeira para o caso geral, ou seja, que a curva $\Omega_{3}$ é tal que: abaixo dessa curva exista apenas uma bifurcação nilpotente degenerada válida e que, entre essa curva e $\Omega_{2}$ existam duas bifurcações nilpotentes degeneradas 
válidas.

Podemos ainda provar mais um corolário:

Corolário 7.9.7. Pelo menos uma bifurcação nilpotente de transição entre o foco nilpotente e a elíptica nilpotente ocorre para parâmetros válidos.

Demonstração. Basta comparar a figura 7.14 com a figura 7.8 .

Isso significa que, de fato, ocorre uma bifurcação mais degenerada no sistema trabalhado. Conjecturamos que essa bifurcação acontece ao longo de uma curva contínua e conexa em $\Delta$. Essa conjectura é baseada em indícios numéricos.

Assim, podemos resumir os principais resultados da secção nas seguintes proposições:

Proposição 7.9.8. Existe uma curva $\Omega_{3}$ de $\Delta$ que está totalmente abaixo de $\Omega_{2}$. Essa curva divide a componente abaixo de $\Omega_{2}$ em duas componentes com diferenças quanto ao diagrama de bifurcações:

- $S e(\delta, \lambda)$ estiver abaixo de $\Omega_{2}$ e acima de $\Omega_{3}$ ocorrem pelo menos dois candidatos a bifurcação nilpotente sendo que todos com parâmetros válidos.

- Se $(\delta, \lambda)$ estiver abaixo de $\Omega_{3}$ ocorrem pelo menos dois candidatos a bifurcação nilpotente, sendo que, pelo menos uma dentre essas bifurcações ocorre para parâmetros não-válidos.

Proposição 7.9.9. Ocorre uma transição de foco nilpotente para elíptica nilpotente com parâmetros válidos.

\subsection{Conclusões e conjecturas do capítulo}

Por fim, vamos enunciar os principais resultados do capítulo, obtidos como corolários das proposições (todos os resultados são válidos em $\mathcal{W} \times \Delta$ ): 
Teorema 7.10.1. Fixado $(\delta, \lambda) \in \Delta$, para todo $0<x \leq \frac{1}{2 \lambda}$ existe um único $y=y_{B T 1}(x, \delta, \lambda)$, um único $\beta=\beta_{t r}\left(x, y_{B T 1}, \delta, \lambda\right)$, um único $\alpha=A\left(x, y_{B T 1}, \beta_{t r}, \delta, \lambda\right)$ e um único $\mu=M\left(x, y_{B T 1}\right.$, $\left.\beta_{t r}, \delta, \lambda\right)$ tal que o ponto $\left(x, y_{B T 1}\right)$ com os parâmetros $\left(A, \beta_{t r}, M, \delta, \lambda\right)$ é um equilíbrio do sistema no qual o jacobiano do sistema é nilpotente. Caso a20 $\neq 0 \neq$ a11, então estes pontos serão bifurcações de Bogdanov-Takens. Em nenhum outro ponto $(x, y, \alpha, \beta, \mu, \delta, \lambda), o$ jacobiano será nilpotente e os parâmetros serão válidos.

Teorema 7.10.2. Existe um conjunto aberto e denso de $\Delta$, que chamaremos de $A$, tal que para todo $\left(\delta_{0}, \lambda_{0}\right) \in A$, caso $\left(x_{0}, y_{0}\right)$ com parâmetros $\left(\alpha_{0}, \beta_{0}, \mu_{0}, \delta_{0}, \lambda_{0}\right)$ seja um equilíbrio com jacobiano nilpotente e a11 $\left(x_{0}, y_{0}, \alpha_{0}, \beta_{0}, \mu_{0}, \delta_{0}, \lambda_{0}\right)=0$, então temos uma bifurcação de Bogdanov-Takens de codimensão três e o sistema localizado no equilíbrio é equivalente a:

$$
\left\{\begin{array}{c}
\dot{x}=y+\mathcal{O}\left(\|(x, y)\|^{5}\right) \\
\dot{y}=x^{2}+E x^{3} y+\mathcal{O}\left(\|(x, y)\|^{5}\right)
\end{array}\right.
$$

com $E \neq 0$.

Teorema 7.10.3. Existe um conjunto aberto e denso de $\Delta$, que chamaremos de $B$, tal que para todo $\left(\delta_{0}, \lambda_{0}\right) \in B$, caso $\left(x_{0}, y_{0}\right)$ com parâmetros $\left(\alpha_{0}, \beta_{0}, \mu_{0}, \delta_{0}, \lambda_{0}\right)$ seja um equilíbrio com jacobiano nilpotente e a20 $\left(x_{0}, y_{0}, \alpha_{0}, \beta_{0}, \mu_{0}, \delta_{0}, \lambda_{0}\right)=0$, então temos uma bifurcação nilpotente (tipo foco, elíptica ou a transição entre ambas) e o sistema localizado no equilíbrio é equivalente a:

$$
\left\{\begin{array}{c}
\dot{x}=y+\mathcal{O}\left(\|(x, y)\|^{5}\right) \\
\dot{y}=-x^{3}+d 11 x y+d 21 x^{2} y+d 31 x^{3} y+\mathcal{O}\left(\|(x, y)\|^{5}\right)
\end{array}\right.
$$

com $d 11<0$ e $d 21 \neq 0$.

Teorema 7.10.4. (Classificação de $\Delta$ ) Existem três curvas que dividem $\Delta: \Omega_{1}, \Omega_{2}$ e $\Omega_{3}$, sendo que $\Omega_{3}$ está sempre abaixo de $\Omega_{2}$. Dessa forma, podemos dividir um conjunto aberto e denso de $\Delta(A \cap B)$ em três componentes conexas, cada uma com um diagrama de bifurcação diferente:

- Acima da curva $\Omega_{1}$, não ocorrem bifurcações de Bogdanov-Takens de codimensão três. 

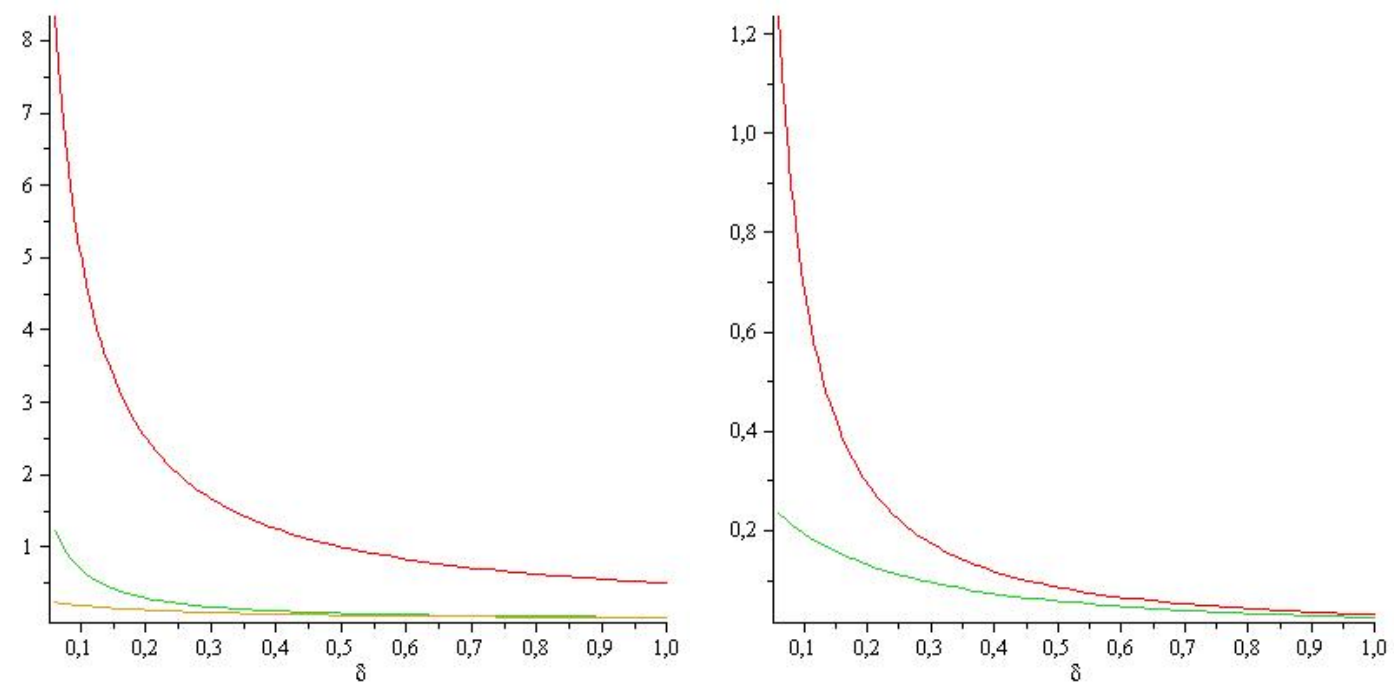

Figura 7.15: À esquerda: A curva acima (vermelho) é $\Omega_{1}$, a do meio (verde) é $\Omega_{2}$ e a de baixo (amarela) é $\Omega_{3}$. À direita: A curva acima (vermelho) é $\Omega_{2}$ e a de baixo (verde) é $\Omega_{3}$. Compare a curva $\Omega_{2}$ com a obtida em [1].

- Abaixo da curva $\Omega_{1}$, ocorre pelo menos uma bifurcação de Bogdanov-Takens de codimensão três.

- Acima da curva $\Omega_{2}$, não ocorrem bifurcações nilpotentes degeneradas.

- Entre a curva $\Omega_{3}$ e $\Omega_{2}$, ocorrem pelo menos duas bifurcações nilpotentes degeneradas, sendo que elas ocorrem para parâmetros válidos (pois $\alpha \geq 0$ ).

- Abaixo da curva $\Omega_{3}$, ocorre pelo menos duas bifurcações nilpotentes degeneradas, sendo que, pelo menos uma dessas bifurcações ocorre para parâmetros não válidos (pois $\alpha<$ $0)$.

\subsection{2}

Teorema 7.10.5. Ocorre uma transição de foco nilpotente para elíptica nilpotente com parâmetros válidos.

Com base em indícios numéricos propomos as seguintes conjecturas: 
- $A=\Delta$.

- $B=\Delta$.

- Entre a curva $\Omega_{3}$ e $\Omega_{2}$, ocorrem duas bifurcações nilpotentes com parâmetros válidos.

- Abaixo da curva $\Omega_{3}$, ocorre apenas uma bifurcação nilpotente com parâmetros válidos. 


\section{Capítulo 8}

\section{Resultados complementares}

Neste trabalho já realizamos um estudo sobre todas as bifurcações dos equilíbrios que podem ocorrer. No entanto, através de duas abordagens diferentes chegamos em resultados complementares. Um dos objetivos deste capítulo é utilizar as informações obtidas através das duas abordagens diferentes e conseguir um resultado complementar sobre o número de equilíbrios que o sistema pode ter: provaremos que, fixados os parâmetros em $\mathcal{W}_{1} \times \Delta$, além de $C$ e a origem, podemos ter: zero, um, dois ou três equilíbrios.

Como segundo objetivo queremos complementar a análise do sistema 1.1. Para isso, apresentaremos resultados de outros autores e um diagrama de bifurcação apresentado em [1]. É importante destacar que o diagrama de bifurcação foi conjecturado através dos mesmos resultados obtidos no presente trabalho, apenas considerando as conjecturas com base numérica, aqui apresentadas, verdadeiras.

\subsection{Número de equilíbrios}

Nesta secção vamos estudar o número de equilíbrios que podem ocorrer no sistema. Em uma primeira abordagem, chegamos a conclusão que, para $(x, y)$ com parâmetros $(\alpha, \beta, \mu, \delta, \lambda)$ ser um equilíbrio, deveríamos ter $F(x, \alpha, \beta, \mu, \delta, \lambda)=0$ e $y=y_{x}(x, \alpha, \beta, \mu, \delta, \lambda)$ (ver capítulo 2). A função $F(x, \alpha, \beta, \mu, \delta, \lambda)$ é um polinômio de grau cinco em $x$ e, portanto, não pode ser resolvida por radicais (de forma genérica). Dessa forma, poderíamos ter até cinco equilíbrios 
válidos para $(\alpha, \beta, \mu, \delta, \lambda)$ fixado.

Note que um equilíbrio não pode desaparecer ou aparecer do sistema sem que isso seja caracterizado por uma bifurcação. As bifurcações desse sistema nas quais isso pode acontecer são: sela-nó e transcríticas.

Assim, vamos dividir este estudo em duas etapas. Em um primeiro momento, estudaremos o número de equilíbrios perto da bifurcação transcrítica de codimensão três. Faremos isso pois esta bifurcação é o único centro organizador do qual surgem equilíbrios no interior de $Q$. Em um segundo momento, trataremos da continuação das superfícies de sela-nó.

Proposição 8.1.1. Fixados $(\alpha, \beta, \mu, \delta, \lambda)$ suficientemente próximos de $T C_{3}$, o sistema 1.1 tem dois equilíbrios nos eixos (origem e $C$ ) e pode ter zero, um, dois ou três equilíbrios no interior do quadrante $Q=\left\{(x, y) \in \mathbb{R}^{2} ; x \geq 0, y \geq 0\right\}$.

Demonstração. Tomemos cortes transversais a $T C_{2}$ suficientemente próximos a $T C_{3}$. Temos apenas duas possibilidades de cortes (ver apêndice sobre $T C_{3}$ ), como vemos nas figuras 8.1 e 8.2. Aproveitamos estas figuras para fazer uma análise das possibilidades do número de equilíbrio $i$ dependendo da região onde o parâmetro se encontra.

Assim, através da análise de possibilidades que fizemos na figura chegamos a conclusão de que o sistema pode ter:

- caso $i=1$, temos $0,1,2$ ou 3 equilíbrios, dependendo dos parâmetros.

- caso $i=2$, temos 1, 2, 3 ou 4 equilíbrios, dependendo dos parâmetros.

- caso $i=3$, temos 2, 3, 4 ou 5 equilíbrios, dependendo dos parâmetros.

Analisando um caso em particular, chegamos a conclusão de que $i=1$ e portanto temos a primeira opção. Por exemplo, calculando $F(x)$ sobre o ponto $T C_{3}$, não obtemos nenhuma raiz $x$ que pertença a ]0, $\frac{1}{\lambda}$ [. Isso significa que a figura 8.1 representa os equilíbrios do sistema próximos a $T C_{3}$. 




Figura 8.1: Primeira possibilidade. Temos duas possibilidades distintas que podem ser vistas com cortes transversais à curva $T C_{2}$. Em cada região, colocamos o número possível de equilíbrios em função de uma das regiões (que recebeu o número $i$ de singularidades). Existe apenas mais uma opção consistente, vista na figura 8.2.

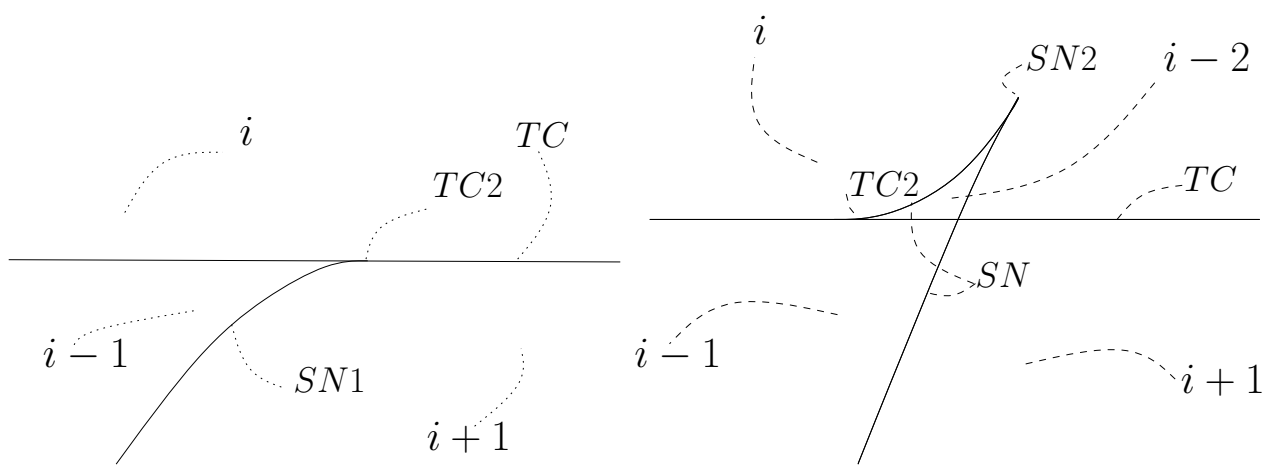

Figura 8.2: Segunda possibilidade. Temos duas possibilidades distintas que podem ser vistas com cortes transversais à curva $T C_{2}$. Em cada região, colocamos o número possível de equilíbrios em função de uma das regiões (que recebeu o número $i$ de singularidades). 
Agora, devemos tratar da continuação da superfície da dobra geral. Fixado $(\delta, \lambda) \in \Delta$, caso esta superfície seja "bem comportada", então a união da dobra geral com o plano transcrítico dividira $\mathcal{W}$ em quatro componentes conexas distintas (já apresentadas na última demonstração) e concluiríamos que existem no máximo três equilíbrios no sistema. Assim, devemos tratar da continuação da superfície de sela-nó e achar as condições necessárias para ela ser "bem comportada".

Lema 8.1.2. A dobra geral cruza o plano transcrítico apenas nas curvas subordinadas a $T C_{3}$.

Demonstração. As equações que devem ser satisfeitas para termos um ponto de sela-nó no interior de $Q$ são:

$$
\begin{aligned}
& F(x, \alpha, \beta, \mu, \delta, \lambda)=0 \\
& F_{x}(x, \alpha, \beta, \mu, \delta, \lambda)=0
\end{aligned}
$$

Além disso, para em um determinado parâmetro ocorrer bifurcação transcrítica, devemos ter:

$$
\alpha=-\frac{(\delta \beta+\delta \lambda-1) \lambda}{\delta}=\alpha_{0}
$$

Substituindo em $F$, podemos resolver em função de $\mu$ :

$$
M_{S N / T C}=-\frac{(x \delta \beta+x \delta \lambda-x+\delta) \delta^{2}}{\left(-\delta-x^{2} \lambda+x^{2} \delta \beta \lambda-x \delta \beta+x^{2} \delta \lambda^{2}\right)^{2}}
$$

que para ser positiva, devemos ter:

$$
x>-\frac{\delta}{\delta \beta+\delta \lambda-1}
$$

Substituindo $\alpha=\alpha_{0}, \mu=M_{S N / T C}$ em $F_{x}$ e considerando apenas os termos que podem se anular obtemos uma expressão quadrática em $x$, onde:

$$
\begin{gathered}
F_{x}\left(-\frac{\delta}{\delta \beta+\delta \lambda-1}\right)=-\delta \\
F_{x}\left(\frac{1}{\lambda}\right)=\frac{3+8 \delta^{2} \lambda^{2}+8 \delta^{2} \beta \lambda-11 \delta \lambda+2 \delta^{2} \beta^{2}-5 \delta \beta}{\lambda}
\end{gathered}
$$

Assim, para haver solução em $x$, deve-se satisfazer duas condições:

$$
\begin{gathered}
\frac{3+8 \delta^{2} \lambda^{2}+8 \delta^{2} \beta \lambda-11 \delta \lambda+2 \delta^{2} \beta^{2}-5 \delta \beta}{\lambda}>0 \\
\frac{1}{\lambda}>-\frac{\delta}{\delta \beta+\delta \lambda-1} \rightarrow-1+\delta \beta+2 \delta \lambda<0
\end{gathered}
$$


Resolvendo $\frac{3+8 \delta^{2} \lambda^{2}+8 \delta^{2} \beta \lambda-11 \delta \lambda+2 \delta^{2} \beta^{2}-5 \delta \beta}{\lambda}=0$ em função de $\beta$ obtemos duas expressões. Cada uma é relativa a um ponto transcrítico de codimensão três (sendo um deles não válido). Analisando o sinal, chegamos à conclusão de que, para que $\mu$ seja positivo, devemos ter $\beta<\frac{-2 \delta \lambda+5 / 4-1 / 4 \sqrt{8 \delta \lambda+1}}{\delta}$. Dessa forma, apenas para $\beta<\beta_{T C 3}$ temos cruzamento da sela-nó com $T C$ fora de $T C_{2}$. Como para cada $(\alpha, \beta)$ fixos, isso ocorre em um único $\mu$, chegamos $\grave{A}$ conclusão desejada.

Assim, sempre que tomarmos uma secção transversal a $T C_{2}$ a superfície de sela-nó cruza no máximo uma vez o plano transcrítico. Dessa forma, se a superfície de dobras for conexa, então como o plano transcrítico divide $\mathcal{W}$ em duas componentes conexas, a superfície de dobras em cada uma dessas componentes será conexa.

Assim, podemos mostrar um resultado suficiente para que a dobra geral seja comportada:

Lema 8.1.3. Fixado $\left(\delta_{0}, \lambda_{0}\right) \in \Delta$ e assumindo que:

- a superfície de dobras é conexa.

- a superfície de dobras não se auto-intersecta.

Então, $\mathcal{W}\left(\mathcal{W}_{1}\right)$ é dividido em (no máximo) quatro componentes conexas.

Demonstração. O plano transcrítico divide $\mathcal{W}\left(\mathcal{W}_{1}\right)$ em duas componentes conexas. Cada uma dessas componentes é homeomorfia a $\mathbb{R}^{3}$.

Sabemos que a superfície de dobras é conexa e fechada em cada uma dessas componentes (lembre-se que a fronteira encontra-se no plano $T C$ ). Sabemos também que a superfície de dobras não é de fato uma superfície, uma vez que tem singularidades (cúspides). No entanto, essas singularidades podem ser regularizadas sem que o seu complementar ganhe novas componentes conexas. Assim, passamos a ter uma superfície $C^{\infty}$ mergulhada (ver capítulo 4), conexa e fechada (logo orientável) em cada uma das componentes de $\mathcal{W}$ divididas por $T C$. Pelo teorema de Jordan-Brouwer diferenciável (ver [17]), essa superfície divide cada uma das componentes conexas de $\mathcal{W}$ em outras duas, obtendo quatro no total. Isso encerra a demonstração. 
No capítulo 6, obtivemos:

- a primeira condição do lema 8.1.3 para $\beta>0$;

- a segunda condição do lema 8.1 .3 para $\beta>0$ e $\delta \lambda>\frac{1}{8}$.

Isso significa que:

Teorema 8.1.4. (Número de equilíbrios) Fixados $(\alpha, \beta, \mu, \delta, \lambda) \in \mathcal{W}_{1} \times \Delta \operatorname{com} \delta \lambda>\frac{1}{8}$, o sistema 1.1 tem dois equilíbrios nos eixos (origem e $C$ ) e pode ter zero, um, dois ou três equilíbrios no interior do quadrante $Q=\left\{(x, y) \in \mathbb{R}^{2} ; x \geq 0, y \geq 0\right\}$.

Em [1] Saleh declara que este resultado é válido para todos os parâmetros em $\mathcal{W} \times \Delta$. Saleh argumenta esse resultado invocando uma análise computacional.

\subsection{Resultados de outros autores}

Nesta secção, queremos completar a análise do modelo. Até agora, fizemos o estudo das bifurcações, mas não nos preocupamos em interpretar os resultados quanto aos equilíbrios, suas estabilidades e possíveis órbitas periódicas. Nesta secção vamos citar os principais resultados obtidos por outros autores de modo a completar ao máximo a análise e possibilitar uma interpretação biológica do modelo.

Começamos citando os resultados obtidos em [1]. Neste trabalho, o autor obtém uma classificação topológica dos equilíbrios do sistema genérico, baseado em dois resultados principais:

- a existência de, no máximo, três equilíbrios no interior do quadrante de interesse biológico;

- a existência de um domínio de captura compacto.

A partir de agora, $X$ vai denotar um campo vetorial nestas condições, onde a origem é um equilíbrio, $C$ é um outro equilíbrio no eixo $x$ e o comportamento no bordo de $Q$ é igual ao do sistema $\left(f_{1}, f_{2}\right)$ 1.1. Lembramos que só estamos interessados em estudar $Q$. 
Com essas premissas, Saleh analisa as configurações topológicas genéricas de um sistema deste tipo. Começamos definindo o que significa ser genérica em nosso caso:

Definição 8.2.1. Um campo de vetores $X$ sobre o plano com um domínio compacto de captura é de Morse-Smale se:

- existe um número finito de equilíbrios e órbitas periódicas, sendo todos hiperbólicos;

- não existem conexões de sela;

Campos de vetores Morse-Smale constituem um conjunto aberto e denso (na devida topologia) do espaço de todos os campos do plano (ver [8] para uma visão superficial ou $[12,13]$ para uma discussão aprofundada). Assim, eles podem ser considerados como campos genéricos.

Note que, mesmo restringindo o campo $X$ ao domínio compacto de nosso interesse, podemos ter uma conexão de sela entre a origem e $C$. No entanto, note que o campo restrito ao bordo do domínio compacto é sempre topologicamente equivalente, não importando o parâmetro tomado. Fazendo as devidas adaptações para esse caso, chegamos a conclusão que o sistema trabalhado poderá ser considerado genérico caso:

- exista um número finito de equilíbrios e órbitas periódicas, sendo todos hiperbólicos;

- não existam conexões de sela no interior de $Q$;

Agora, vamos lembrar dois teoremas que são importantes para os resultados subsequentes. O primeiro é o conhecido teorema de Poincaré-Bendixson. Antes de enuncia-lo, lembremos o seu objeto de estudo (vide [11]):

Seja $X: \Omega \rightarrow \mathbb{R}^{2}$ um campo vetorial definido em $\Omega$ aberto de $\mathbb{R}^{2}$, de classe $C^{k}(k \geq 1)$. Seja $\phi(t)=\phi(t, p)$ a curva integral do campo $X$ passando pelo ponto $p \in \Omega$, definida no seu intervalo máximo $I_{p}=\left(\omega_{-}(p), \omega_{+}(p)\right)$. Se $\omega_{+}(p)=+\infty$, defini-se:

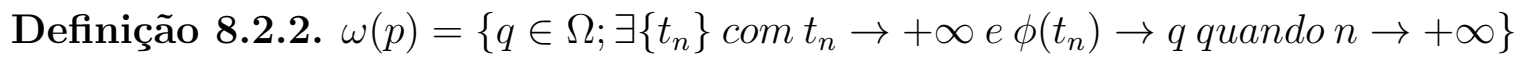
Assim: 
Teorema 8.2.3. (Poincaré-Bendixson) Seja $X: \Omega \rightarrow \mathbb{R}^{2}$ um campo vetorial definido em $\Omega$ aberto de $\mathbb{R}^{2}$, de classe $C^{k}(k \geq 1)$. Seja $\phi(t)=\phi(t, p)$ uma órbita desse campo definida para todo $t \geq 0$ e tal que $\phi(t) \in K$ compacto para todo $t \geq 0$. Nesse caso, se $\omega(p)$ possuir um número finito de equilíbrios ocorre uma das seguintes alternativas:

- $\omega(p)$ contém apenas pontos regulares, então $\omega(p)$ é uma órbita periódica;

- $\omega(p)$ contém apenas pontos singulares, então $\omega(p)$ é um equilíbrio;

- $\omega(p)$ contém pontos regulares e singulares, então $\omega(p)$ consiste em um conjunto de órbitas, cada uma das quais tende a um ponto singular do próprio conjunto $\omega(p)$ quanto $t \rightarrow \pm \infty$.

Já mostramos que o campo $X$ que estamos trabalhando se encaixa nesse caso. Além desse teorema, também temos interesse em estudar os equilíbrios através da teoria de índice. Lembremos que o índice de um equilíbrio $p$ hiperbólico vale 1 caso $p$ seja um atrator ou repulsor e -1 caso seja uma sela. Além disso, o índice em um conjunto $D$ vale:

$$
i_{D}(X)=\sum_{X(p)=0, o \in \operatorname{int}(D)} \operatorname{ind}_{p}(X)
$$

Assim:

Teorema 8.2.4. (Poincaré-Hopf) Nestas condições, caso o campo X seja tangente ou transversal a $\partial D$, então:

$$
i_{D}(X)=\chi_{D}
$$

onde $\chi_{D}$ é o número de Euler de D.

Com esses teoremas, Saleh classifica as configurações topológicas possíveis de $X$. Para isso, Saleh começa fazendo uma operação (que ele chama de cirurgia) para "retirar"' órbitas periódicas. Essa operação consiste em tomar anéis maximais de órbitas periódicas (sem equilíbrios) e fazer transformações no campo de forma a obter um outro campo genérico (que chamaremos de $Y$ ) no qual o anel desaparece, mas o comportamento fora do anel permanece igual. Em outras palavras, se $A$ é esse anel, $\mathcal{O}$ a componente exterior do anel e $\mathcal{J}$ a parte interior do anel, então $\left.X\right|_{\mathcal{O}}=\left.Y\right|_{\mathcal{O}}$ e $\left.X\right|_{\mathcal{J}}= \pm\left. Y\right|_{\mathcal{J}}$, enquanto $\left.Y\right|_{A}$ não possui órbitas 
periódicas. Dessa forma, ele argumenta que pode considerar o sistema sem órbitas periódicas.

Feita essa cirurgia, chamaremos o novo campo de $Y$. Baseados nos dois teoremas anteriores, é possível provar que:

Proposição 8.2.5. Tomando um campo de vetores $X$ Morse-Smale, após realizarmos a cirurgia, $Y$ têm uma das seguintes configurações de equilíbrios, sem considerar a origem e $C:$

a Se C for um atrator, então existem zero ou dois equilíbrios. No caso de haver dois equilíbrios, podemos ter uma sela $S$ e um atrator $A$ ou uma sela $S$ e um repulsor $R$.

b Se C for uma sela, então existem um ou três equilíbrios. Sempre existe um atrator $A_{0}$ e, no caso de existirem outros dois equilíbrios, teremos uma sela $S$ e um atrator $A$ ou uma sela $S$ e um repulsor $R$.

Proposição 8.2.6. (Classificação sem ciclos-limites) Nas condições da proposição anterior e assumindo que $X$ não possua ciclos limites, temos seis possibilidades de espaços de fase, listados na figura: 1.5 .

Proposição 8.2.7. Nas condições da proposição 8.2.5, dado o tipo de espaço de fase em $Y$, um anel maximal pode ser reinserido de forma a recuperar $X$ da seguinte forma:

- No caso $[a]$ :

- Nenhum anel possível;

- Um anel em torno de A;

- Um anel em torno de R;

- No caso $[b]$ :

- Um anel em torno de $A_{0}$;

- Um anel em torno de $A_{0}$, ou de $A$ ou em torno de todos os equilíbrios $A_{0}, S$ e $A$; 



Figura 8.3: Acima: Possíveis configurações dos equilíbrios quando $C$ é um atrator. Abaixo: Possíveis configurações dos equilíbrios quando $C$ é uma sela. $A$ denota atratores, $R$ repulsores e $S$ selas. Figura reproduzida de [1]. 
- Um anel em torno de $A_{0}$, ou de $R$ ou em torno de todos os equilíbrios $A_{0}$, $S$ e $R$;

Cada anel pode conter um número finito de órbitas periódicas (incluindo zero). O tipo topológico de $X$ só depende do tipo topológico de $Y$.

Além dessas proposições obtidas em [1], existe um outro teorema complementar dessa análise apresentado em [5]:

Teorema 8.2.8. O sistema $X=\left(f_{1}, f_{2}\right) 1.1$ possui, no máximo, duas órbitas periódicas em $Q$.

\subsection{Diagrama de Bifurcação}

Vamos apresentar o diagrama de bifurcação do modelo, que foi primeiro desenhado em [1]. Ressaltamos que os resultados utilizados por Saleh em [1] para desenhar os diagramas de bifurcação foram corroborados no presente trabalho.

Apresentaremos dois diagramas de bifurcação de $\mathcal{W}$ : um deles para $(\delta, \lambda)$ abaixo da curva $\Omega_{2}$ e a outra acima (ver 1.2). Isso decorre do fato de em cada um destes conjuntos haver diferentes tipos de centros organizadores. Além da divisão pela curva $\Omega_{2}$, também fizemos outras divisões de $\Delta$. No entanto não vamos considera-las, uma vez que elas não alteram a existência dos centros organizadores, mas apenas suas validades.

Em cada um destes diagramas tomamos alguns planos de especial interesse, onde conjecturamos o diagrama de bifurcação global do sistema. Na figura acima de 1.2 tomamos quatro planos de interesse, sendo três deles entre os quatro centros organizadores e um deles que corresponde a $\alpha=0$. Neste caso, o modelo passa a ter uma função resposta do tipo Holling II e o diagrama de bifurcação deve ser comparado com o estudo de Bazykin (ver [8]). Já na figura abaixo de 1.2, tomamos dois planos de interesse, sendo um deles correspondente a $\mu=0$. Neste caso, estamos no modelo estudado por Zhu em [6]. Os diagramas de bifurcação em cada um desses planos podem ser conjecturados numericamente através do programa Matcom e geram as figuras 8.5, 8.6 e 8.7. 



Figura 8.4: Acima $(\delta, \lambda)$ abaixo da curva $\Omega_{2}$. Abaixo, $(\delta, \lambda)$ acima da curva $\Omega_{2}$. Figura retirada de $[1]$. 


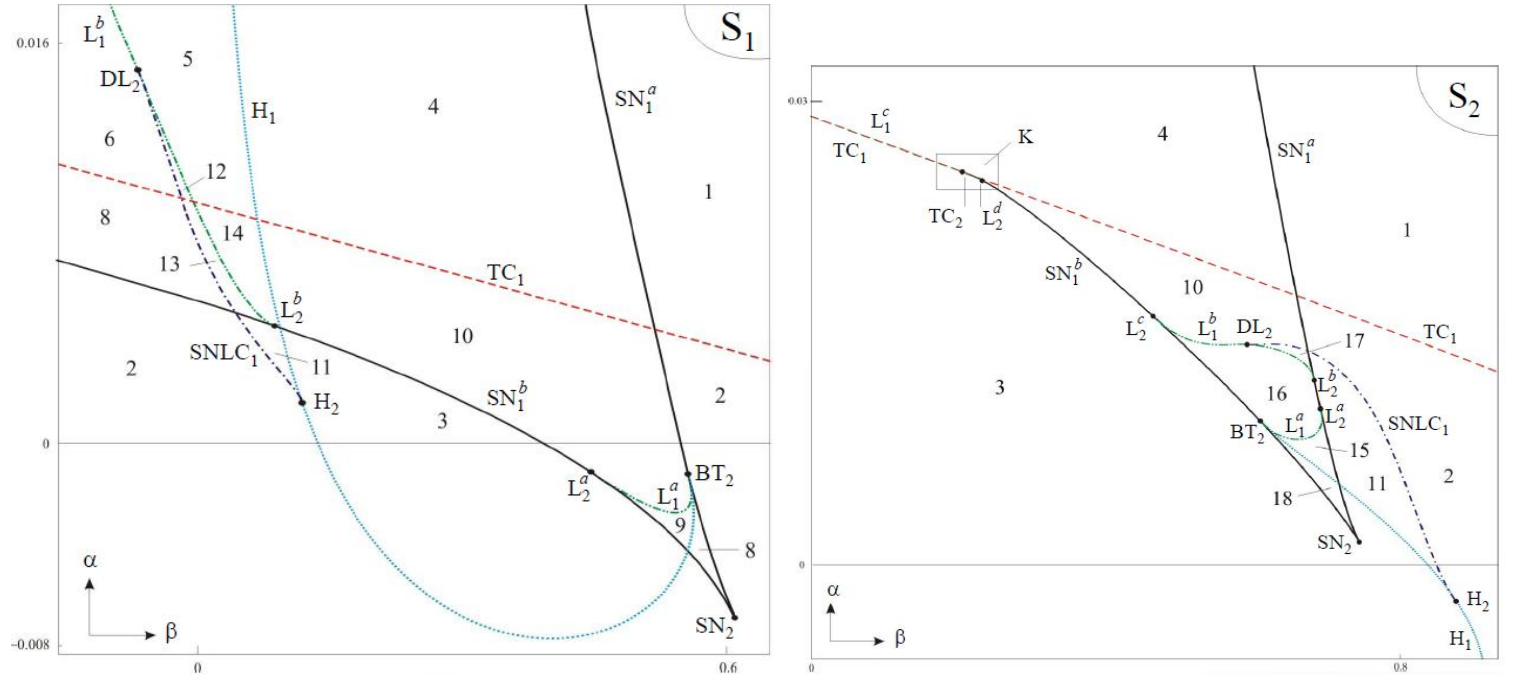

Figura 8.5: Planos $S 1$ e $S 2$. Figura retirada de [1].

Em cada uma das componentes das figuras 8.5, 8.6 e 8.7, colocamos um número referente à configuração topológica do sistema naquele estrato. Essas configurações podem ser vistas em 8.8. 

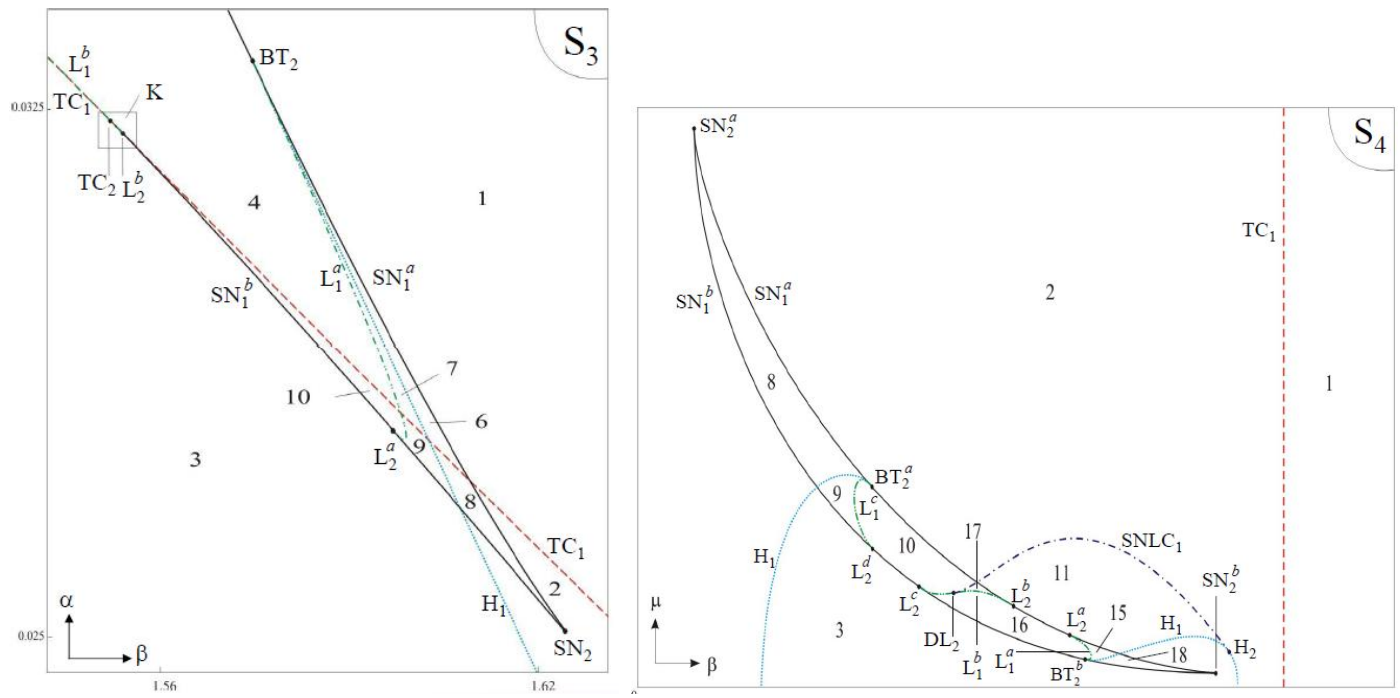

Figura 8.6: Planos $S 3$ e $S 4$. Figura retirada de [1].
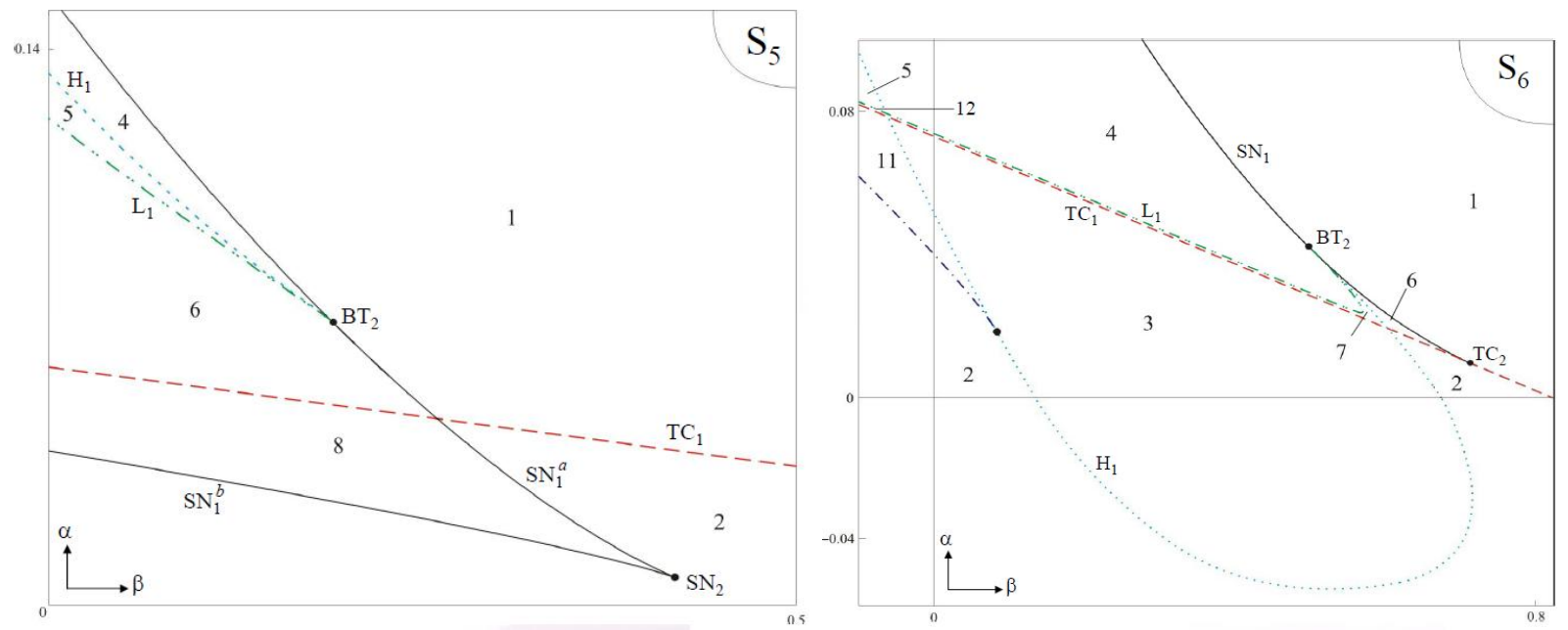

Figura 8.7: Planos $S 5$ e $S 6$. Figura retirada de [1]. 


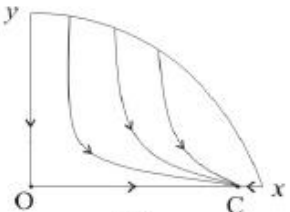

(1)

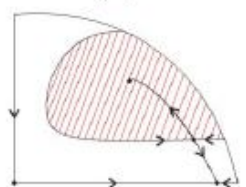

(6)

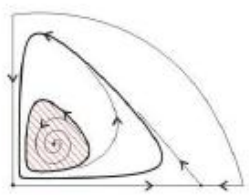

(11)

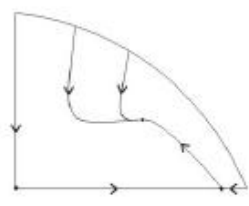

(2)

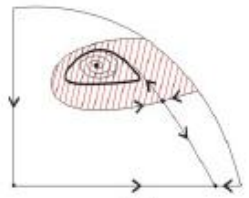

(7)

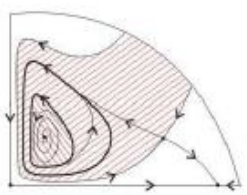

(12)

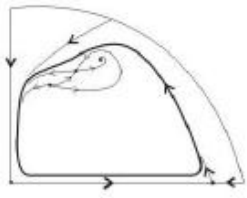

(16)

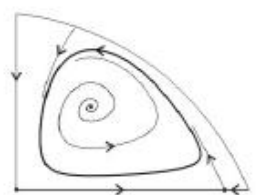

(3)

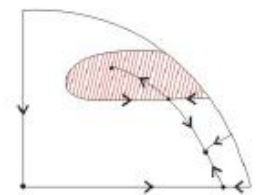

(8)

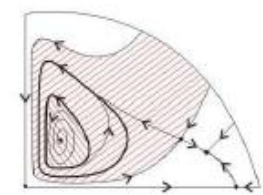

(13)

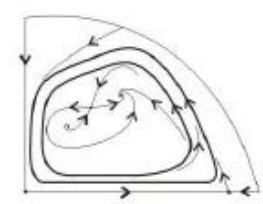

(17)



(4)

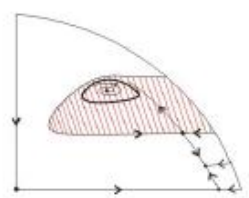

(9)

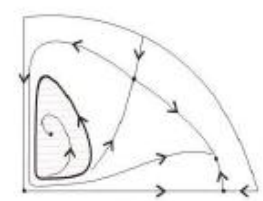

(14)

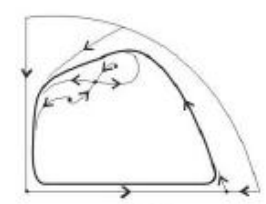

(18)

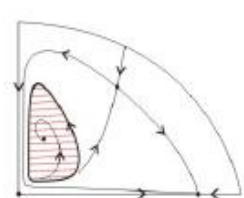

(5)

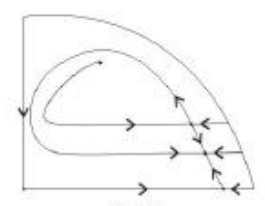

(10)



(15)

Figura 8.8: Espaços de fase relativos a cada estrato (genérico) das figuras 8.5, 8.6 e 8.7. Figura retirada de [1]. 


\section{Capítulo 9}

\section{Conclusões}

Nesta dissertação trabalhamos com o seguinte sistema:

$$
\left\{\begin{array}{c}
\dot{x}=x\left(1-\lambda x-\frac{y}{\alpha x^{2}+\beta x+1}\right)=f_{1}(x, y, \alpha, \beta, \mu, \delta, \lambda) \\
\dot{y}=y\left(-\delta-\mu y+\frac{x}{\alpha x^{2}+\beta x+1}\right)=f_{2}(x, y, \alpha, \beta, \mu, \delta, \lambda)
\end{array}\right.
$$

onde os parâmetros podem pertencer a:

- $\mathcal{W} \times \Delta$ ou

- $\mathcal{W}_{1} \times \Delta$.

onde

$$
\begin{gathered}
\Delta=\left\{\left((\delta, \lambda) \in \mathbb{R}^{2} ; \delta>0, \lambda>0\right\}\right. \\
\mathcal{W}=\left\{(\alpha, \beta, \mu) \in \mathbb{R}^{3} ; \alpha \geq 0, \mu \geq 0, \beta>-2 \sqrt{\alpha}\right\} \\
\mathcal{W}_{1}=\left\{(\alpha, \mu, \beta) \in \mathbb{R}^{3} ;(\alpha, \beta, \mu) \in \mathcal{W}, \beta>0\right\}
\end{gathered}
$$

Para estudar as bifurcações fizemos uma divisão do espaço de parâmetros. O ponto mais interessante desta divisão é a forma como o problema pode ser reinterpretado: uma família a dois parâmetros $(\Delta)$ de sistemas tri-paramétricos $(\mathcal{W})$. Dessa forma, não só conseguimos achar os tipos de bifurcações do sistema, mas também ter uma ideia do comportamento qualitativo dessas bifurcações no espaço de parâmetros.

Para organizar esta análise, estudamos os centros organizadores. Vale ressaltar que para 
achar a posição e o tipo dos centros organizadores usamos apenas álgebra e análise, mas para achar a quantidade de centros organizadores em cada ponto de $\Delta$ e para ter uma ideia qualitativa do diagrama de bifurcação recorremos ao cálculo numérico e à simulação computacional.

Os resultados finais, apresentados de forma organizada, encontram-se nas próximas duas secções.

\subsection{Resultados válidos para $\mathcal{W} \times \Delta$.}

Os principais teoremas provados para esse domínio são:

Teorema 9.1.1. (Dominio de captura) Para $(\alpha, \beta, \mu, \delta, \lambda) \in \mathcal{W} \times \Delta$ e para todo $p \in \mathbb{R}$ tal que $p>\frac{1}{\lambda}\left(\frac{1}{4 \delta}(1-\delta)^{2}+1\right)=f(\delta, \lambda)$ o dominio compacto $B_{p}=\{(x, y) \| x+y \leq p, x \geq 0, y \geq 0\}$ é invariante por $X_{t}$ com $t>0$.

Teorema 9.1.2. (Plano Transcrítico) Para todo $(\delta, \lambda) \in \Delta$ o conjunto de bifurcação do sistema em $\mathcal{W}$ associado ao ponto $C=\left(0, \frac{1}{\lambda}\right)$ possui um ponto transcrítico de codimensão 3 $\left(T C_{3}\right)$, duas semi-retas conexas transcríticas de codimensão $2\left(T C_{2}\right)$ subordinadas a $T C_{3} e$ dois semi-planos conexos transcríticos de codimensão 1 (TC $\left.C_{1}\right)$ subordinadas a $T C_{2}$ e $T C_{3}$. A união de $T C_{1}, T C_{2}$ e $T C_{3}$ é um plano em $\mathcal{W}$ que chamaremos de $T C$. Os conjuntos $T C_{1}$, $\mathrm{TC}_{2}$ e $\mathrm{TC}_{3}$ s ão não vazios para todos os $(\delta, \lambda) \in \Delta$. Não ocorrem mais degenerescências.

Teorema 9.1.3. (Divisão de $\Delta$ ) Existem três curvas que dividem $\Delta$ : $\Omega_{1}, \Omega_{2}$ e $\Omega_{3}$, sendo que $\Omega_{3}$ está sempre abaixo de $\Omega_{2}$. Dessa forma, podemos dividir um conjunto aberto e denso de $\Delta$ em três componentes conexas, cada uma com um diagrama de bifurcação diferente:

- Acima da curva $\Omega_{1}$, não ocorrem bifurcações de Bogdanov-Takens de codimensão três.

- Abaixo da curva $\Omega_{1}$, ocorre pelo menos uma bifurcação de Bogdanov-Takens de codimensão três.

- Acima da curva $\Omega_{2}$, não ocorrem bifurcações nilpotentes. 
- Entre a curva $\Omega_{3}$ e $\Omega_{2}$, ocorrem pelo menos duas bifurcações nilpotentes, sendo que elas ocorrem para parâmetros válidos (pois $\alpha<0$ ).

- Abaixo da curva $\Omega_{3}$, ocorre pelo menos duas bifurcações nilpotentes, sendo que, pelo menos uma dessas bifurcações ocorre para parâmetros não válidos.

\subsection{2}

Teorema 9.1.4. No sistema 1.1 ocorrem as seguintes bifurcações:

- transcrítica de codimensão um, dois e três.

- Sela-nó de codimensão um e dois.

- Hopf.

- Bautin.

- Bogdanov-Takens.

- Bogdanov-Takens de codimensão três.

- foco nilpotente.

- elíptica nilpotente.

- transição entre foco nilpotente e a elíptica nilpotente.

Listamos a seguir propriedades para as quais temos indícios numéricos, mas não temos demonstrações algébricas.

- Não ocorre bifurcação do tipo sela-nó de codimensão maior do que dois.

- Não ocorre bifurcação do tipo Hopf de codimensão maior do que dois.

- As bifurcações de Bautin formam uma curva regular em $\mathcal{W}$.

- Só ocorrem as bifurcações listadas em 9.1.4.

- No teorema 1.3.2 o aberto denso mencionado é igual a $\Delta$. 
- Abaixo de $\Omega_{2}$, para cada $(\delta, \lambda)$ fixado, existe apenas duas bifurcações nilpotentes.

- Abaixo de $\Omega_{1}$, para cada $(\delta, \lambda)$ fixado, existe apenas uma bifurcação Bogdanov-Takens de codimensão três.

\subsection{Resultados demonstrados para $\mathcal{W}_{1} \times \Delta$.}

Aqui, além de todos os teoremas anteriores, provamos os seguintes resultados:

Teorema 9.2.1. Para $(\delta, \lambda) \in \Delta$ fixos, o plano $T C_{1}$ cruza $\mathcal{W}_{1}$ caso $\delta \lambda<1$, a reta $T C_{2}$ cruza $\mathcal{W}_{1}$ caso $\delta \lambda<\frac{1}{2}$ e o ponto $T C_{3} \in \mathcal{W}_{1}$ caso $\delta \lambda<\frac{3}{8}$.

Teorema 9.2.2. (Número de equilíbrios) Fixados $(\alpha, \beta, \mu, \delta, \lambda) \in \mathcal{W}_{1} \times \Delta \operatorname{com} \delta \lambda>\frac{1}{8}$, o sistema 1.1 tem dois equilíbrios nos eixos (origem e $C$ ) e pode ter zero, um, dois ou três equilíbrios no interior do quadrante $Q=\left\{(x, y) \in \mathbb{R}^{2} ; x \geq 0, y \geq 0\right\}$.

É interessante que se diga que o segundo teorema dessa secção parece ser válido para $\mathcal{W}$. Em [1] formula-se esse resultado como válido para todo $\mathcal{W}$ e, para isso, demonstra-se a conexidade da superfície de dobras (sela-nó e suas degenerescências) utilizando técnicas computacionais.

\subsection{Problemas que ficaram em aberto}

Após feito esse estudo, é imediato imaginar os novos desafios. Primeiramente, deixamos algumas conjecturas ao longo do trabalho que se baseiam em indícios numéricos e que reforçam o formulado em [1]. Seria interessante demonstrar ou refuta-las. Acreditamos que essas conjecturas poderiam ser provadas utilizando Análise Intervalar [16]. Uma demonstração analítica ou algébrica também não é descartada, mas esbarra na dificuldade de trabalhar com equações muito longas.

Além disso, achamos uma bifurcação de codimensão quatro (ver capítulo 7.7) que, até nosso presente conhecimento, não foi estudada. O simples desafio de entender o comportamento dessa bifurcação já é matematicamente relevante. Além disso, esse conhecimento poderia ser 
especialmente interessante para o estudo do diagrama de bifurcação global do sistema 1.1.

Por fim, estudar as bifurcações das órbitas periódicas do sistema é um desafio sem nenhuma abordagem algébrica ou analítica que conheçamos até o momento (em [1] ele trata apenas tangencialmente desse problema, sem se aprofundar nele). Mesmo se acreditássemos que todas as bifurcações desse tipo sejam subordinadas a uma bifurcação de equilíbrios, não seria fácil de estabelecer o diagrama completo de bifurcações. Provavelmente um trabalho numérico seria muito mais rico de conclusões e conjecturas do que um trabalho puramente algébrico e analítico. O presente trabalho poderia servir como apoio a um trabalho desse gênero.

\section{4 Últimas considerações}

Ao longo do trabalho, achamos duas discrepâncias com [1]:

- mais divisões em $\Delta$, ocasionada pela demonstração da existência das bifurcações de codimensão três, mas não necessariamente com parâmetros válidos (ver capítulo 7.9).

- a existência de uma bifurcação de codimensão quatro.

No entanto, nenhuma delas causa grandes alterações nos diagramas de bifurcação encontrados em [1].

Além disso, as interpretações biológicas não são alteradas. Demonstramos que pode ocorrer uma bi-estabilidade em sistemas desse tipo (sem extinção do predador), e que, dependendo das condições iniciais, uma órbita tenderá para um ou outro equilíbrio (ou órbita periódica). Além disso, dependendo dos parâmetros chegamos à conclusão que as seguintes configurações devem ocorrer:

- Extinção dos predadores.

- Extinção dos predadores ou coexistência entre presas e predadores, dependendo das condições iniciais. 
- Coexistência de presas e predadores, não importando a condição inicial.

- Dupla possibilidade de coexistência entre presa e predador.

No mais, o método de estudo através dos centros organizadores se mostrou eficiente, uma vez que, ao analisarmos a configuração das bifurcações de codimensão mais alta, obtivemos excelentes informações sobre o comportamento das demais bifurcações e sobre o comportamento do sistema de forma geral, como pode ser visto no capítulo 8.

Acreditamos que o presente estudo da uma contribuição relevante para o entendimento deste modelo, corroborando independentemente, em grande parte, o trabalho realizado em [1]. 


\section{Capítulo 10}

\section{Apêndice}

Neste capítulo queremos apresentar a bifurcação de Bogdanov-Takens e as bifurcações de codimensão três que aparecem no trabalho. As últimas tem especial importância por serem os centros organizadores do sistema 1.1. Para cada uma delas, apresentaremos a forma normal e os seus diagramas de bifurcação.

Observação 10.0.1. Em cada uma das figuras que apresentaremos existirá uma notação para as bifurcações globais diferente. Colocaremos as devidas notações na legenda da figura. Isso ocorre pois cada uma das figura foi retirada de um trabalho diferente.

\subsection{Transcrítica de codimensão 3}

Tomemos a seguinte forma normal:

$$
\left\{\begin{array}{c}
\dot{x}=-x \\
\dot{y}=y\left(a+b y+c y^{2}-y^{3}\right)
\end{array}\right.
$$

onde $(a, b, c) \in \mathbb{R}^{3}$ são os parâmetros. Então o equilíbrio $(0,0)$ com os parâmetros $(0,0,0)$ é um ponto de bifurcação transcrítica de codimensão três. Está bifurcação possui uma reta de bifurcação transcrítica de codimensão dois e duas curvas de sela-nó de codimensão dois subordinada a ela. Uma das curvas de sela-nó de codimensão dois é associadas a bifurcações em equilíbrios localizados em $y<0$. Dessa forma, para o nosso caso, temos apenas uma 


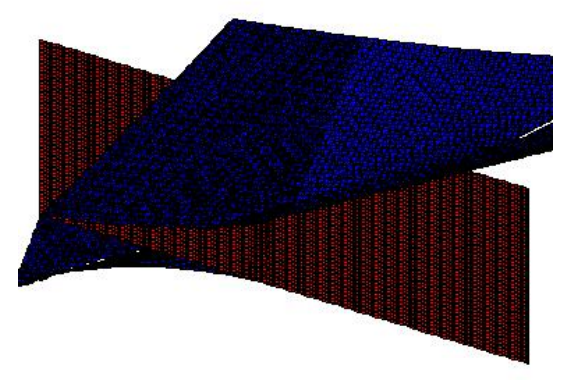

Figura 10.1: Em azul as superfícies sela-nó e em vermelho a superfície transcrítica.

curva de sela-nó de codimensão dois associada a ela. Veja a figura 10.1 para uma ilustração.

\subsection{Bogdanov-Takens}

Tomemos a seguinte forma normal:

$$
\left\{\begin{array}{lc}
\dot{x}= & y \\
\dot{y}= & \beta_{1}+\beta_{2} x+x^{2}-x y+\mathcal{O}\left(|(x, y)|^{3}\right)
\end{array}\right.
$$

onde $\left(\beta_{1}, \beta_{2}\right) \in \mathbb{R}^{2}$ são os parâmetros. Então o equilíbrio $(0,0)$ com os parâmetros $(0,0)$ é um ponto de bifurcação de Bogdanov-Takens (codimensão dois). O diagrama de bifurcação pode ser visto na figura 10.2 .

\subsection{Bogdanov-Takens degenerada}

Tomemos a seguinte forma normal:

$$
\left\{\begin{array}{lc}
\dot{x}= & y \\
\dot{y}= & a+b x+x^{2}+c x y+x^{3} y+\mathcal{O}\left(|(x, y)|^{5}\right)
\end{array}\right.
$$

onde $(a, b, c) \in \mathbb{R}^{3}$ são os parâmetros. Então o equilíbrio $(0,0)$ com os parâmetros $(0,0,0)$ é um ponto de bifurcação de Bogdanov-Takens de codimensão três. Para apresentar o diagrama 

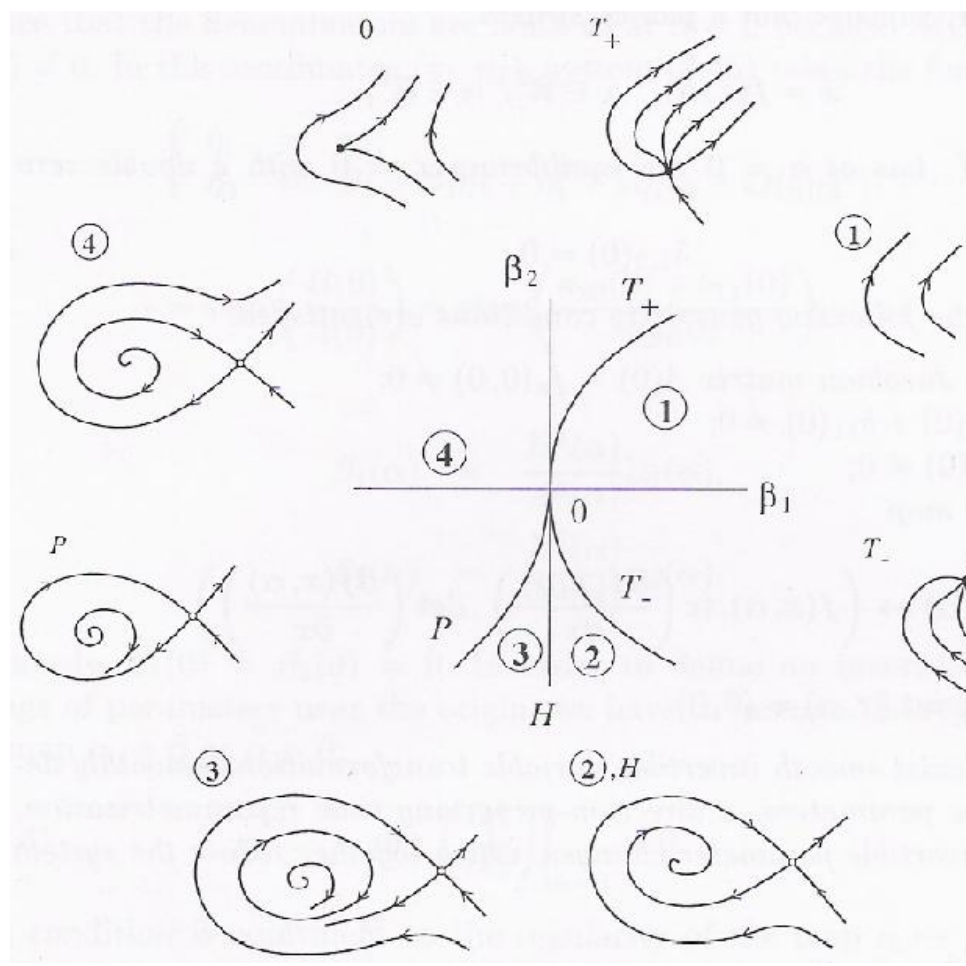

Figura 10.2: Diagrama de bifurcação do Bogadanov-Takens. $P$ representa as bifurcações homoclínicas. Figura retirada de [8]. 


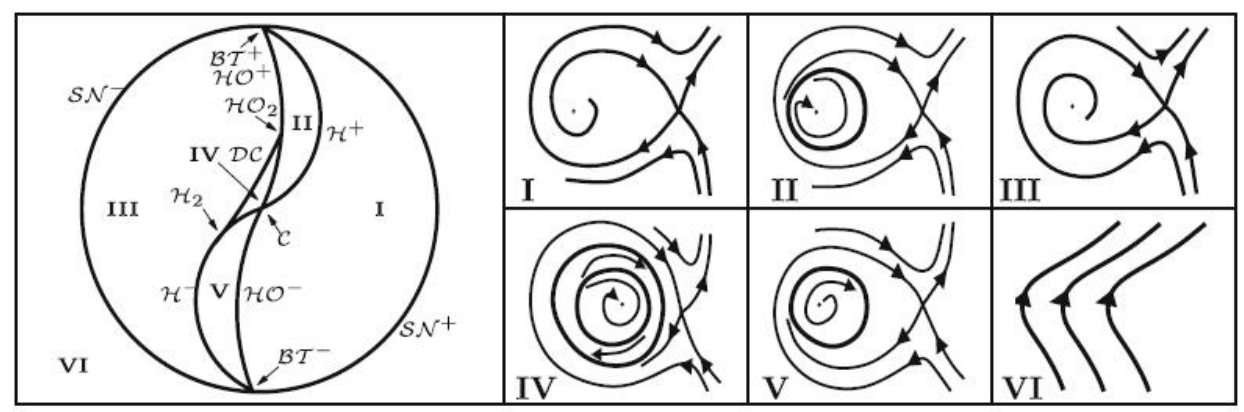

Figura 10.3: Diagrama de bifurcação do Bogadanov-Takens de codimensão três. HO representa as bifurcações homoclínicas. Figura retirada de [7].

de bifurcação tomamos uma bola de raio $\epsilon$ centrada no ponto de bifurcação e planificamos a superfície desta bola. O raio $\epsilon$ é suficientemente pequeno. Ver a figura 10.3.

\subsection{Foco-Nilpotente}

Tomemos a seguinte forma normal:

$$
\left\{\begin{array}{lc}
\dot{x}= & y \\
\dot{y}= & a+b x+c x^{2}+e x y+x^{3}-x^{2} y+x^{3} y+\mathcal{O}\left(|(x, y)|^{5}\right)
\end{array}\right.
$$

onde $(a, b, c) \in \mathbb{R}^{3}$ são os parâmetros e $e \neq 0$. Então, caso $e<2 \sqrt{2}$ o equilíbrio $(0,0)$ com os parâmetros $(0,0,0)$ é um ponto de bifurcação foco nilpotente. Para apresentar o diagrama de bifurcação tomamos uma bola de raio $\epsilon>0$ centrada no ponto de bifurcação e planificamos a superfície desta bola. O raio $\epsilon$ é suficientemente pequeno. Ver a figura 10.4.

\subsection{Elíptica-Nilpotente}

Tomemos a seguinte forma normal:

$$
\left\{\begin{array}{lc}
\dot{x}= & y \\
\dot{y}= & a+b x+c x^{2}+e x y+x^{3}-x^{2} y+x^{3} y+\mathcal{O}\left(|(x, y)|^{5}\right)
\end{array}\right.
$$

onde $(a, b, c) \in \mathbb{R}^{3}$ são os parâmetros e $e \neq 0$. Então, caso $e>2 \sqrt{2}$ o equilíbrio $(0,0)$ com os parâmetros $(0,0,0)$ é um ponto de bifurcação elíptica nilpotente. Para apresentar o 


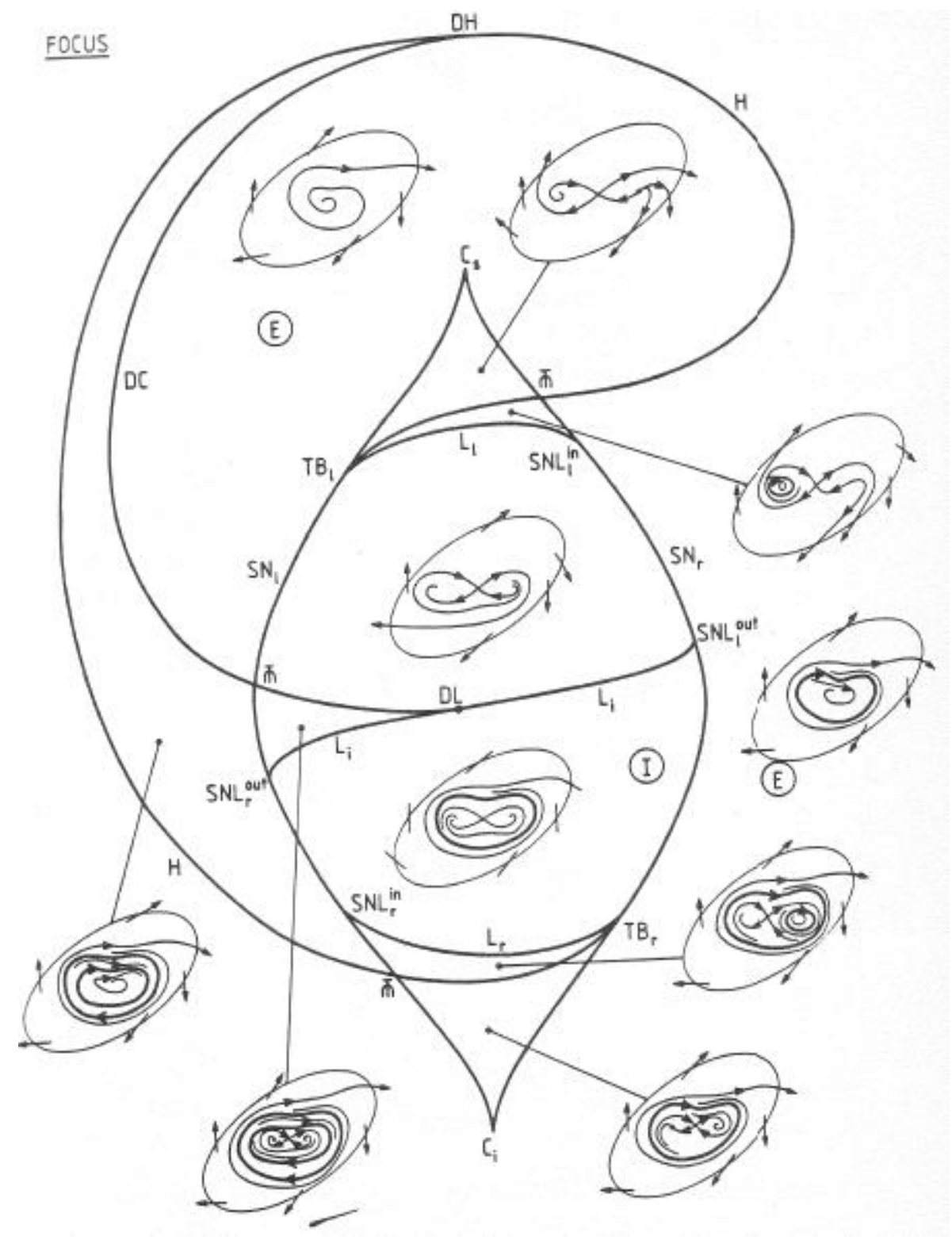

Figura 10.4: O diagrama de bifurcação do foco nilpotente. $L$ são as bifurcações homoclínicas e $D C$ é uma sela-nó de órbitas periódicas. Figura retirada de [10]. 
diagrama de bifurcação tomamos uma bola de raio $\epsilon>0$ centrada no ponto de bifurcação e planificamos a superfície desta bola. $\epsilon$ é tomado tão pequeno quanto se queira. Ver a figura 10.5. 


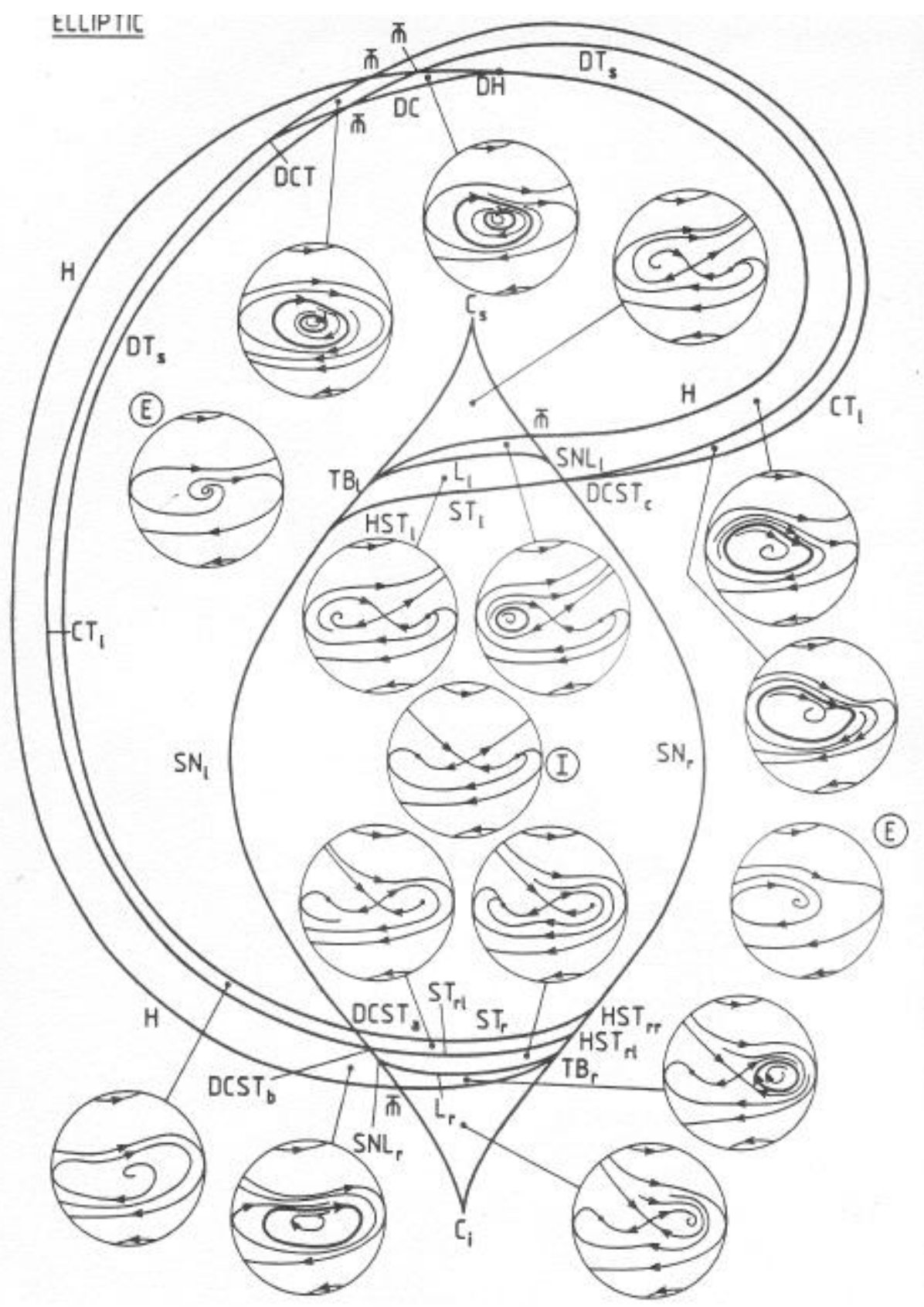

Figura 10.5: O diagrama de bifurcação da elíptica nilpotente. $L$ são as bifurcações homoclínicas, $D C$ é uma sela-nó de órbitas periódicas, $C T$ é um ciclo tangente, $S T$ uma separatriz de sela tangente e $D T$ uma dupla tangência. Figura retirada de [10]. 


\section{Referências Bibliográficas}

[1] K. Saleh, Organising centres in semi-global analysis of dynamical systems, PhD Thesis, University of Groningen 2005.

[2] Broer, H. W.; Saleh, K.; Naudot, V.; Roussarie, R. Dynamics of a predator-prey model with non-monotonic response function. Discrete Contin. Dyn. Syst. 18 (2007), no. 2-3, 221-251

[3] Broer, H. W.; Naudot, V.; Roussarie, R.; Saleh, K. A predator-prey model with non-monotonic response function. Regul. Chaotic Dyn. 11 (2006), no. 2, 155-165

[4] Broer, H. W.; Naudot, Vincent; Roussarie, Robert; Saleh, Khairul Bifurcations of a predator-prey model with non-monotonic response function. C. R. Math. Acad. Sci. Paris 341 (2005), no. 10, 601-604.

[5] Henk W. Broer, Valery A. Gaiko, Global qualitative analysis of a quartic ecological model, Nonlinear Analysis 2009.

[6] H. Zhu, S. A. Campbell e G.S.K. Wolkowicz, Bifurcation analysis of a predatorprey system with nonmonotonic functional response, Siam J. Appl. Math. 63 (2002), 636-682.

[7] Lamontagne, Yann; Coutu, Caroline; Rousseau, Christiane Bifurcation analysis of a predator-prey system with generalised Holling type III functional response. J. Dynam. Differential Equations 20 (2008), no. 3, 535-571

[8] Y. Kuznetsov, Elements of Applied Bifurcation Theory, Springer, 3rd edition, 2004 . 
[9] F. Dumortier, R. Roussarie e J. Sotomayor, Generic 3-parameter families of vector fields on the plane, unfolding a singularity with nilpotent singular part. The cusp case of codimension 3, Ergod. Th \& Dynam. Sys. 7 (1987), 375-413.

[10] F. Dumortier, R. Roussarie, J. Sotomayor e H. Zoladek, Bifurcations of planar vector fields, LNM 1480, Springer Verlag 1991.

[11] Sotomayor, Jorge, Equações Diferenciais Ordinárias, Projeto Euclides, 1979.

[12] Sotomayor, Jorge Curvas definidas por equações diferenciais no plano, Colóquio Brasileiro de Matemática, Instituto de Matemática Pura e Aplicada, Conselho Nacional de Desenvolvimento Científico e Tecnológico, Rio de Janeiro, 1981.

[13] J. Palis e W. de Melo, Geometric Theory of Dynamical System, Springer-Verlag 1982.

[14] R. Levins, The strategy of model building in population biology, American Scientist 54 (1966): 421-431.

[15] H. Weiss, A mathematical Introduction to Population Dynamics, 27 Colóquio Brasileiro de Matemática, IMPA, 2009.

[16] Montanher, Tiago M., Métodos intervalares em optimização global, dissertação de mestrado, IME-USP, 2009.

[17] Lima, Elon Lages, Análise Real: v3, Análise vetorial, Coleção matemática universitária, IMPA, 2008. 